\title{
ACKNOWLEDGEMENTS
}

\section{FAMILY NUMBER NON-CONSERVATION INDUCED BY THE SUPERSYMMETRIC MIXING OF SCALAR LEPTONS*}

\author{
Michnel Jeffrey Saul Levin: \\ Stanford Linear Accelerator Canter \\ Stenford Univertiky \\ Suplord, Californiz 9usos
}

August 1987

Prepared for the Department of Enercy under contratt number DE-ACU3-76SFO05I5

Printed in the United States of Americe. Avaifable from the National Texhnt(A) Information Service, U.S. Departmest of Commerce, 5285 Port Roysl Roud, Springfield, Virginie 22161. Price: Printed Copy A36, Mikrobsthe A01.

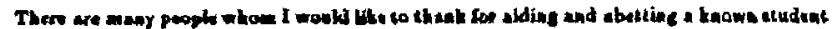

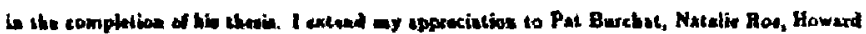

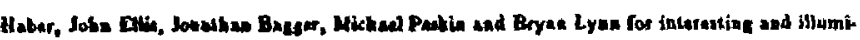

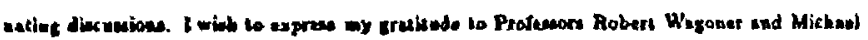

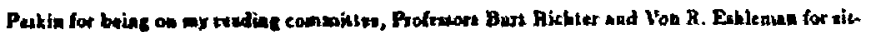

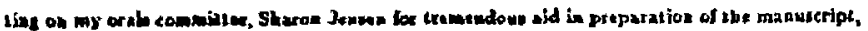

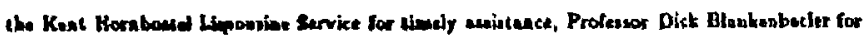

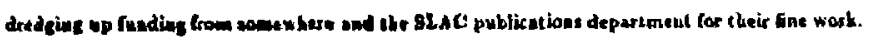

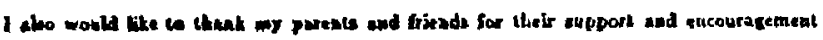

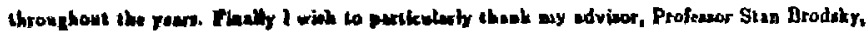

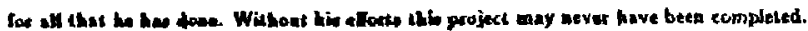

\section{DISCLAIMER}

This report wil prepared as an scocunt of wart tpansored by an agency of the United Slates Covernsoenl. Neltber the Unived Stalca Gowerament aor any apency thereof, nor any of their

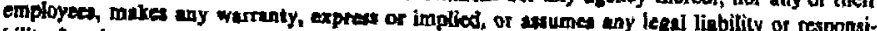

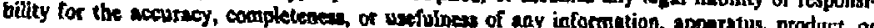

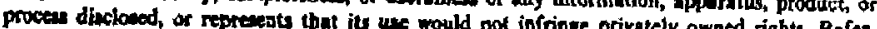
enco hereio to any jpecific commercial produch, pracesa, ar servioe by trade neme, trademark, manufacturer, or otherwive dop col necturily constitute of imply its eadortement, recom-

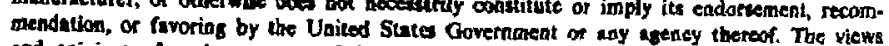
and opinions of erition by the Uailed States Government of any agency thereof. The views Vaitad Stakes Goverament or any acency tbertor.

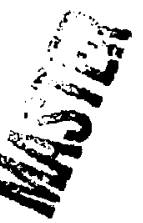




\section{TABLE OF CON'IENTS}

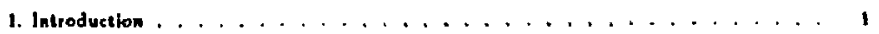

2. Contribution to Anomaloug Mrzzntic Monent front slepton Mixing . . . . . . 4

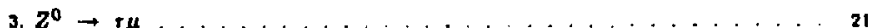

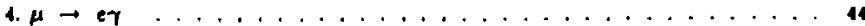

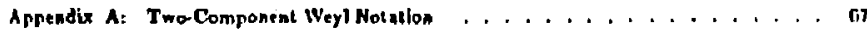

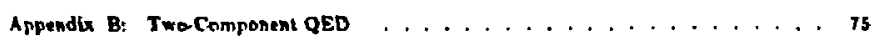

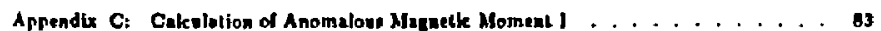

Appendix D: Cukulation of Anomulant Matnetk Mtoment II . . . . . . . . . 04

Appendix E: Superoymmetrie QED contribullos to Anomalows Moment . . . . . . III

Appendix F: Contribution to Anomalow Moment from Smeon Mfixing . . . . . 124

A ppendix O: Plote Related to Anomalous Noment . . . . . . . . . 135

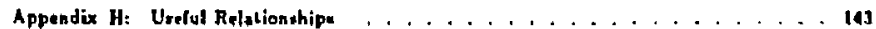

Appendix 1: The Supery)nmetric Lagranghn $\ldots \ldots \ldots \ldots$ t58

Appendix J: Mixing Matrieies and Mane Eicenvelder ............ 220

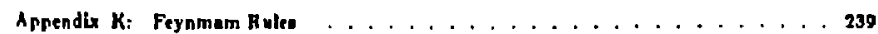

Appendix $L:$ Computstion of $Z^{0} \rightarrow \tau \mu$ Matrix Elements $\ldots \ldots 257$

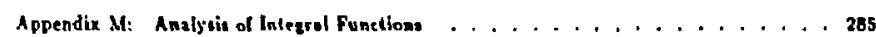

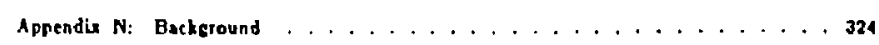

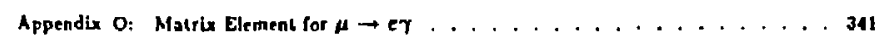

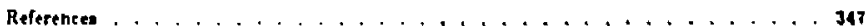

\section{TABLES}

Table 3.1: Fielde Appearing in Chapter Three ... . . . . . . . . 39

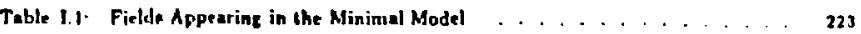

Table C. I: Integral Functions . . . . . . . . . . . . . 272 


\section{Introduction}

\subsection{WHY SUPERSYMMETAY?}

Many of the oriainal motivations for auperuymmatry have long since vaniahed. It wae recognized lmmediately that ateh a aymmetry must be ceverely brakan in the low energy world. In its early days auperaymmetry wee bolked on principelly - i hopeful solution to the gauge hhararchy probleze (the quation of why thero should exist such a diacrepancy between the two energy acale $M_{\text {mon }}$ at $100 \mathrm{GoV}$, and $M_{G U T}$ at $10^{36}$ geV or more). In order to be weeful in thie respect the ecale at which supersymmetry brents cannot exceed the went ecale by more than an order of maenitude if the breaking mechanitem is to be relatively aimple. Thus we heve replaced one cymmetry-breaking hierurcby problem by another. Admittedly the radiative hierarchy problem in realvad but thin was but a chimeral problem to begin with (presumably if we posensed the mathematkal tockniquen, and the ataspina, wo solve the theory to all ordern, including sos-perturbativa effects, wo would only have to adjust the overall ecales onco). Eurby eaplrations of uniting known partieles into supermultiplets falled utterly with the secult of an lantantaneous doubline of the number of atalm conjectured to exint. None of then naw atates has yet been esen.

Despite this the superaymmetric principle be become increavingly populer. The true resson for this is the most compelling of motivations, that same antetic belief in unification which apawned the Grand Unified Theorim, and therefore the zauge hieraschy problem. Supenymmetric theortu are believed to be the oaly theories capable of unifying fundamental botonic and fermionic telde. Thin is undoubtedly due to our imperfect understanding of what apin ie at a fundamental, topological, level. Surely uniting then two dieparnte anctors of the rzalerial world is at least as profound as considering ever-grenter gauge groups to fragment.

When supersymmetry is raised to a local symmetry the Lorents group manifests itself as a subgroup. Thus gravitation becomes incorporated into the theory in a natural way. In addition, the currently popular atring models find the inclusion of supersymmetry highly desirable, 4 not irrecusable.

\subsection{The Family Puzzle}

One of the oututandias puzster in tementary particle phyaics is that of particle genetation. The material constituente of the world, as opposed to the medialors of the fundamental forces, appear to be replicaled in families or zeneratione. Each earm to be a simulecrum of the previou famlly, but of grenter mwe. At this time thres such generations are known. Why this thould occur is - complate myatary.

This myotery is parteularly profound in the ketonic, or weakly Interacting, sector where each family would enem to poeses an absolutuly conserved quantum number, zenerally referred to a electron, muon and tau number or, collectively, a family number. In all reactione whkh have been thus far observed the amount of "electron-nus" hese aeither increased nor decroaed. This is in atark contract to the quark (atrongly-imleracting) enctor where miring between the otatea b known to oceur at a fundamantal keve. In the light of the "atandard model" it is understood why this should be the case. In extenotions beyood this it in frequently difficult to ratain this cymmetry in an sppealine fahion.

A gymmetry may be realled beally or slobully. U local wuch a "horizontal" symmetry would have profound Implication, sequiring a new family-changing sauge cector. A slobal aymmetry, if not the coneequent of some local symmetry, but if impoed or maintained arificlally (for intance by constraining come paramelet to be amall) $\boldsymbol{l}$ to be viewed $a$, at beat, unnatural and probably untenable. Contidered in thie light it in interesting to ponder whether lepton family number might not be broken to nome amall degree. We will discuss a mechanism which might accomplish this. 


\subsection{SCALAR LEPTON MiXING}

The most egrugious upect of $(\mathrm{N}=1)$ superoymmetric theories is that each particle alate is accompanied by a 'ruper-partner', alate with identical quantum numbers axve that it differs in spin by one half unit. For the leptons these are scalars and are called "sleptons", or acalar leptons. Theae consist of the charged sleptons (selectron, smuon, atav) and the acalar neutrinos ('eneutrinos').

We examine a model of supersymmetry with woft breaking term in the electrowent sector. Explicit mixing amons the ecalar leptons results in a number of effects, principally non-conservation of lepton family number. Comparison with experiment permits us to plece constraints upon the model.

Each eupersymmetric family has charged aleptons associated with both the left and right-handed helicity st ates of the corresponding lepton. These, 100, may combine (within a single seneration). The effect or thin will be discunsed in shapter two in the context of the anomalous masnetic moment of the muon and the electson. These were selected due to the extremely precine knowledse of their experimental values. In chopter three we will exmine the rasult of intergenerational slepton-mixing for the process $e^{+} e^{-} \rightarrow Z^{0} \rightarrow y^{ \pm} \mu^{\mp}$. Since several $Z^{0}$-factorics, in particular the SLC, are expected to produce these particles copiously, this process, should it occur at a ressonable rate, fould prove experimentally interestins. These matlers will be discussed. In chapter four we considet the elassic family number violating proceas, radiative muon decay: $\mu \rightarrow$ er. These three chaptera are followed by a utring of appendlces, most of which are associated with a particulas chapter. These are referted to within the chapters and contain detaits and derivations not necessary to the cursory understanding of a civen chapter.

\section{Contribution to Anomalous Magnetic} Momenl from Slepton Mixing

\subsection{INTRODUCTION}

In Chapter 1 we saw that the sleptons might mix amont themselves in a supernymmetrit theory. In this chapter we illustrate the manner in which the enomalous magnetic moments of the electron $\left(a_{r}\right)$ and muon $\left(\alpha_{\mu}\right)$ are altered when the theory is mede supersymmetric in a minimal way. We then permit the Ieptons to mix logether which will leed to new eficuts. We will discover, despite the fact that $a_{\mathrm{q}}$ has been determined experimentelly to much greater accuracy than $a_{n}$, that the results of the $\sigma_{p}$ calculation tend to place the mote stringent restrictions on supercymmetry parameters when compared with experiment.

We will confine ourselves to pure QED and SQED (oupersymmetric QED) In this chapler. The extension to the full electroweak theory is atraightforward but tedious. Exactly how this should be done will be made obvious in the later chaptere and their corresponding appendicen.

The calculation will be perlormed in a comewhet pedazozical fashion. As two-component notation is natural for superaymetric calculatione some basic nolation ha bees introduced in Appendix $A$. In Appendix B we examine QED in its two and four component forms and consider the interpretation of two component propagators. Thus armed, In Appendix $\mathrm{C}$ we calculste $a_{\mathrm{e}}$ in fourcomponent form and in Appendix D we repeat the calculation in two-component form to that they may be carefully compared. In Appendix E we next compute $\Delta a_{e}$, the additional contribution from superaymmetrizing the theory (atandard SQED), before the aleptons have been allowed to mix. The moat important contribution from the mixing of the sleptons cones from $\tilde{e}_{L}-\tilde{e}_{R}$ mixing as detailed in Appendix F. If we permit intergenerational mixing then the contribution to a, will be spread over a greater number of kesas. For this reason we restrict ourselves to a single zeneration here. This hat the added benefit of reducing 25 
angles :-1d phase to three angle fote per generation assuming three genorationj). The reveree of this argument will be used in the pext chapter when the loft-right mixing will bo ignored in favor of the intercenerational mixiac. The penultimate appendix (Appendix G) related to chapter two will show plols of the verious results. In Appendix $\mathbf{H}$ we dorive $\mathrm{A}$ few useful formules. In the firat section certain Feynman integrals, which eppear frequently in these pages, are presented. In the second eaction wa diecuse generulized versions of the Gordon Decomposition, a generally useful racult.

As can be resdly seen most of the actual work will be presented in the appendicen. This chapter is primarily a summery of the resulte, comparison with experiment, consequences end commentary. In Section 2.2 we review the concept of anomalous magnetic moments. In 2.3 the results of the appendix calculations are summorized and compered with experiment in 2.4

\subsection{THE MAGNETIC MOMENT}

From - Hamiltonian viewpoint the only interaction between the $\psi$ (electron)

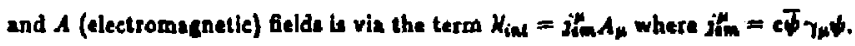
The Gordon Decomposition (Appendix H) can be uad in conjunction with the Dirac equation in thpresence of an $A$-Afld $(\mathcal{H}+m) \psi=0$ where $D_{\mu}=a_{\mu}+i c A_{\mu}$, to divide the current into two pieces. ${ }^{14}$ In the non-ralativiatic limit the frat roduces to the electric charce current density of non-relativhtic quantum mechanice. It is the other term which interests us. This yields an Interaction piece

$$
X_{i=1}^{m+\infty}=j_{\mu} A^{\mu} \sim c / 4 m \bar{\psi} F^{\mu \nu} \sigma_{\mu \nu} \psi+\text { Lotal derivative. }
$$

Non-relativistically

$$
\bar{\psi} F^{\mu \nu} \sigma_{\mu \nu} \psi 2 \psi^{+} g \cdot B_{\sim} \psi
$$

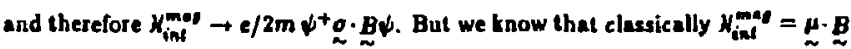
where $\underset{\sim}{\mu}$ is the magnetic moment of the electron. Thus we can identify $\mu=2 / \tilde{m} S$ or, in ereater senerality, $\mu=g e / 2 \mathrm{~m} S$ where $g$ is the cyromegnetic ratio which bere in two." Clasicalby in determined by the charge current diatribution of an infiniterimal current bop (megnetle dipola). $=2$ correaponds to a point dipole. Devintione from $=2$ are civen by $s=2(1+a)$ where $a$ in called the enomalous megnetic moment.

From the two-component composition of the $\bar{\psi}^{\prime \prime} \gamma^{\mu} \psi^{*}$ end $\bar{\psi}$ o $^{\mu \nu} \psi$ vertices (see Appendix B) we mey obsorve thelr belichy itructure. Ignoring the mus of the

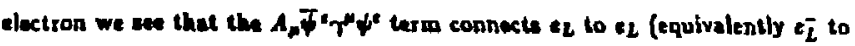

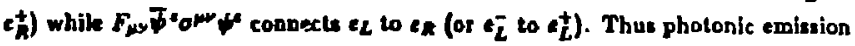
vis the electric charge current leaves the bolicity of the atate unchanged while the megnetic dipole interaction sipe the belicity.

Such obervation have been made in conjunction with the lowest-order vertex (w Illustrated in Fis. 2.10). The ons-loop QED verlex correction bs $w$ in the clasic infundibular (triengle) diegram of Fis. $2.1 \mathrm{~b}$. This will contribute the interactions

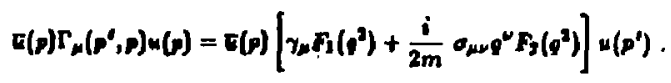

$F_{1}\left(q^{2}\right)$ contribute to the (infinite) wavefunction renormalization while, on shell $\left(g^{2}=0\right), F_{2}\left(s^{2}\right)$ contributes to the magnetic moment and thus to $a_{c}=\left(a_{c}-2\right) / 2$. Indeed, comparing the definition of $a_{e}$ we see that $a_{2}=F_{3}\left(q^{2} \rightarrow 0\right)$. Since QED is a renormalizable theory, and no $F_{3}$-type term appeare in the QED Lagrangian, we know that the $F_{2}$ term reaulting from arat order contributions must be finite (since there is no base parameter to absorb an infinity). The anomalous moment can be thought of a measuring an effective radius of the charge current distribution engendered by radiative corrections to the simple bare "point" vertex.

- Here $A-c=1$ wo thes of is wails of Bolur Megnetone. Otherwien $\underset{\sim}{\mu}=g e h / 2 m e$. 
It is often the case than when we consider interactions at large enetgies, or have particles in internal loops whose inusues are large with reapect to the external lepton masses, we will find that the magnetic $\left(\sigma^{\mu \nu}\right)$ contribution to the cross uection will be reduced relative to that of the electric $(7)$ term (by $\left.O\left(m_{\text {lepen }} / s^{3}, m_{\text {hary }}\right)\right)$ and can thus often be ignored. If for some reason, such a rauge invariance, the $\gamma^{*}$ term cannot appeas then the interaction must go throush the convective ( $\left.\sigma^{m \nu}\right)$ term. We will see this happen when we examine $\mu \rightarrow$ ej on thell $\left(\varphi^{9}=0\right)$ but not lor $Z^{0} \rightarrow r^{+} \mu^{-}\left(\varphi^{2} \neq 0\right)$.

\subsection{REsULTs}

In this wection the resulu of computing the various contributions to $a_{\mu}=$ $\left(g_{\mu}-2\right) / 2$ and $a_{r}=\left(a_{0}-2\right) / 2$, algebraically detailed in the frat few appendices, are ingeminated. We work to oneloop $(\alpha)$ order. The one-loop vertex correction from puse QED (Appendices 2 and 3 ) yielda

$$
a_{1}=a_{\mu}=\frac{a}{2 \pi}
$$

When eupersymmetric QED (SQED) contributione (asuming no mixins amongst the sleptonic atates) are appended, as in Appendix $\mathrm{D}$, we discover the additional contribution

$$
\Delta a_{t}=\frac{a}{2 \pi}\left(I_{2}+I_{n}\right)
$$

where

$$
I_{L, A}=\int_{0}^{1} d x \int_{s}^{1} d x \frac{z(1-s)}{-x^{2}+\frac{m_{e}^{2}-m_{e_{t, n}^{2}}^{2}+m_{\lambda}^{1}}{m_{e}^{2}} z-\frac{m_{l}^{l}}{m_{l}^{2}}}
$$

$m_{e}$ is the mass of the electron.

$m_{\lambda}=m_{i}$ is the mass of the photino.

$m_{\tilde{e}_{L}}$ are the wasses of the left and right selectron.

$$
\text { For } \Delta a_{\mu,}, m_{e} \text { is replaced by } m_{\mu} \text { and } m_{\tilde{e}_{L, n}} \text { by } m_{\tilde{w}_{L, n}} \text {. }
$$

We remask on tome of the limiting cases which are presented in the appendix.

A tikely limil is $m_{\tilde{z}_{\mathrm{c}, n}}>m_{\lambda}>m$ (equation E.24). Then

$$
\Delta a_{e}=\Delta d^{\left(m_{1}=0\right)}=-\frac{a}{12 \pi}\left\{\frac{m_{e}^{2}}{m_{E_{L}}^{2}}+\frac{m_{\epsilon}^{2}}{m_{E_{n}}^{\frac{1}{E_{n}}}}\right\} .
$$

This in preeisely Fayet's 1974 result. "'I we relax the condition that $m_{\lambda}<m_{\tilde{e}_{2}}$ we find (eqn E.32) that, to the next order in $m_{L} / m_{i_{L}, n^{*}}$.

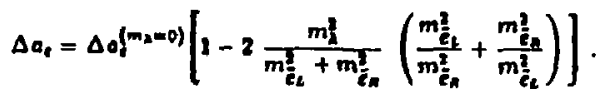

In limits where cuperaymmetry remains unbroken $m_{\ell_{k}}=m_{z_{n}}=m_{e}$ and $m_{\lambda}=0$. In this llmit we find that $\Delta a_{a}=-\alpha / 2 \pi$ wo that, for $(2,1)$,

$$
a_{1}=a_{\varepsilon}^{Q E D}+\Delta a_{1}=0
$$

This is gratifying as we might have antielpated that thete would be no anomalous magnetic moment to any order in an exactly supersymmetric theory (for the eame reason that loop divertences cancel\}. This was fist demonstrated by Ferrara and Remidi. ${ }^{\text {(1) }}$

We now consider the efrect of permitting the left and right aleptons to mix. Indeed, in realistic models with Higes fields, it is often difficult to prevent them from doing so." Since these atates differ in hyperchurce and weak isospin we wee that $S U(2) \times U(1)_{y}$ b broken, although $U(1)_{\text {so }}$ b not. (This way be induced both radiatively ${ }^{(1)}$ and at the tree Jevel. ${ }^{\text {(o) }}$ / Most treatments only consides the case where the photino is mass]ess or nearly so. We thall leave $m_{\lambda}$ as a free 
paremeter." This introduce a number of important new effects which would appear in a complete electroweal treatment ${ }^{|7|}$ and thus represente a suitable decoction but with fewer complication.

Let us first consider muors. The mas eigenutete may be taken to be

$$
\begin{aligned}
& \tilde{\mu}_{2}=\cos \phi \tilde{\mu}_{L}+\sin \phi \tilde{\mu}_{z} \\
& \tilde{\mu}_{2}=-\sin \phi \tilde{\mu}_{L}+\cos \phi \tilde{\mu}_{L} .
\end{aligned}
$$

It is found (in Appendix F) that, to lowest order in $m_{2}^{2} /\left(m_{2}^{2}-m_{\lambda}^{2}\right)$.

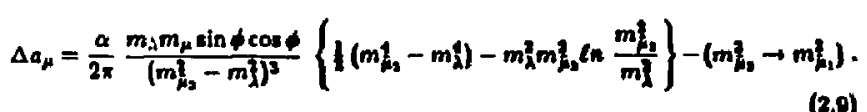

We can write (2.0) as

$$
\Delta a_{\mu}=C\left(f\left(X_{2}\right)-J\left(X_{1}\right)\right)=\Delta e_{\mu}^{2}-\Delta a_{\mu}^{2}
$$

where we have let

$$
\Delta a_{\mu}^{i}=C /\left(X_{i}\right)
$$

and

$$
\begin{array}{rlrl}
x_{i} & =\frac{m_{i}}{m_{\lambda}^{2}} \\
f(x) & =\frac{1\left(x^{2}-1\right)-x \ln x}{(x-1)^{3}} & f(1)=\frac{1}{6} \\
c & =\frac{a \sin \phi \cos \phi}{2 \pi} \frac{m_{\mu}}{m_{\lambda}} & f^{\prime}(1)=-\frac{1}{12} .
\end{array}
$$

\footnotetext{
- Thase are estrophysical bounde on $m_{\lambda}$ but these se model dependent.
}

Note that $\Delta a_{\mu}^{i}$ is periectly regular at $m_{f_{i}}=m_{\lambda}$ and in given by

$$
\left|\Delta \alpha_{\mu}^{i}\right|=\frac{1}{6} c=\frac{\alpha}{12 \pi} \frac{m_{\mu}}{m_{\lambda}} \sin \phi \cos \phi\left|m_{\mu_{\mu}}=m_{\lambda}>m_{\mu}\right| .
$$

The important point is to nole that $\Delta \varkappa_{\mu} \sim m_{\mu} / m_{s u s y}$ here wherea, prior to slepton mixing, we find that $\Delta \mathrm{e}_{\mu} \sim\left(\mathrm{m}_{\mu} / \mathrm{msusy}^{2}\right)^{2}$. The salience of this become apparent when we make contact with experimental limits in the next section. When the anomalows moment in linearly dependent upon the lepton mess we have substantially greater censitivity to new of ects. Concomitant with this atatement. W the asuertion that we can place more stringent limits on the supersymmetry paramelate of the theory. It may be noted, however, that the Feynman diagrams which are responible for the $\mathrm{m}_{\mu}^{2}$ terme ure still present (Diagrams $1,11, \mathrm{ll}$, $\mathrm{N}$ in Fig. F.2 of Appendix $F)$ and there are term of $O\left(m_{\mu}^{2}\right)$ which have been dropped in (2.0) (ces equation F.15) $T$ be $O\left(m_{\mu}^{2}\right)$ term is (for $\left.m_{n}<m_{\tilde{\mu}_{1},}, m_{\lambda}\right)$

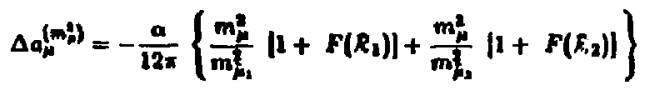

$$
\begin{aligned}
& F(x)=x\left(6 x^{2}-0 x+2\right)+x^{2}(1-x)^{2} \ln \left(\frac{x-1}{x}\right) \\
& l_{1,2}=\frac{m !}{m_{\lambda}^{2}-m_{1,2}^{2}}
\end{aligned}
$$

which can become dominant if $\mid \|$ or $|\pi-0| \leqslant 0\left(m_{\mu} / m_{S U S Y}\right)$. In the limit that $m_{\bar{j}_{1,4}}>m_{\lambda}(2.16)$ becomes

$$
\Delta a_{\mu}=-\frac{a}{12 \pi}\left\{\frac{m_{\mu}^{2}}{m_{\mu_{1}}^{2}}+\frac{m_{\mu}^{2}}{m_{h_{2}}^{2}}\right\}
$$

which is ensentially (2.5) and would give similax limite for slepton masses. For the remainder of this section we will restrict our attention to the terms which are liness in $m_{\mu}$. 
Appendix $G$ contuins verious plots related to $\Delta a_{\mu}$. In Fiz. G.1 $\frac{t}{t} \Delta a_{\mu}^{i}$ is plotted againat $X_{i}$ (as define in (2.12)). We see that $t \Delta a_{j}$, decreasea mono tonleally from \& at $x_{i}=0$ to about 0.1 at $x_{i}=2$ and thence asymptotically to zero. Since $\Delta a_{\mu}=\Delta a_{\mu}^{2}-\Delta a_{\mu}^{1}$ we have plotted $t \Delta a_{\mu}$ versus $X_{1}$ for $m_{p_{1}}=1.01 m_{B_{1}}$ and $m_{B_{2}}=2 m_{D_{2}}$ in figures $G .2$ and G.3 reapectively. Wie note the eeneral feuture of a meximum for $\left|\Delta a_{\mu}\right|$, call it $\left|\Delta a_{\mu}\right|_{\text {max, }}$, which occura at

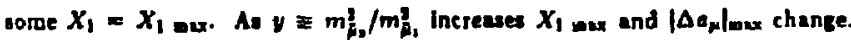

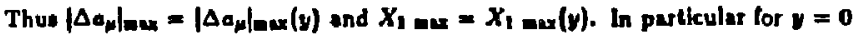

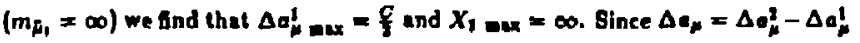
we wee that $X_{1}$ axx $(y)=(1 / y) X_{1}$ au( $(1 / v)$ and $\Delta a_{p} \max (v)=-\Delta a_{p}$ ax $(1 / y)$. Thus $X_{1}=x=0$ for $y=\infty$.

The value of $\left|\Delta a_{\mu}\right|_{\text {wa }}$ is impottant in that it will place the most atringent limits on the supersymunetric parameters. The maxima occur at elutions to the dimentionless transcendental equation (here $I_{i}=X_{i}$ )

$$
\begin{aligned}
& \ln x_{1}= \\
& \frac{y^{\prime}\left(\left(x_{1} y+5\right)\left(x_{1} y-1\right)-2\left(2 x_{1} y+1\right) \ln y_{j}^{\prime}\left(x_{1}-1\right)^{4}-\left(x_{1}+5\right)\left(x_{1}-1\right)\left(x_{1} y-1\right)^{4}\right.}{\left.2 \mid y\left(2 x_{1} y+1\right)\left(x_{1}-1\right)^{4}-\left(2 x_{1}+1\right)\left(x_{1} y-1\right)^{4}\right]}
\end{aligned}
$$

(where $y \neq 1$ when $s_{1}=1$ )

$$
x_{1}=\frac{m_{j_{1}}^{2}}{m_{1}^{2}} \quad x_{1}=\frac{m_{i_{1}}^{2}}{m_{2}^{2}} \quad y=\frac{x_{2}}{x_{1}}=\frac{m_{i_{1}}^{2}}{m_{i_{1}}^{2}}
$$

In Fiz. G. 4 we have plotted $X_{1}$ max as a funetion of $y$ whereas $t \Delta \Delta_{\nu}$ wax versus $y$ appeart in Fig. G.5-6. H must always be remembered that $C \sim 1 / m_{\lambda}$. Unsurprisingly the maximal value achievsble by $\left|\Delta a_{\mu}\right|$ is $\mid C$ at $y=0$ and $y=\infty$ (at which points $X_{1}$ ans $\left.=\infty, 0\right)$. Thus $\frac{d}{c}\left|\Delta a_{\mu}\right|$ achieves its grealest value when $m_{\tilde{\mu}}$. (ot $\left.m_{\tilde{\mu}_{2}}\right) \rightarrow 0$ while $m_{\tilde{\mu}_{1}}$ (or $\left.m_{\tilde{\mu}_{1}}\right) \rightarrow \infty$. Since $C \sim m_{\mu} / m_{\lambda}$ we expect that $\left|\Delta a_{\mu}\right|$ is meximized when the supersymmetric messes are as amall a possible and the mast-apliting in the aleptonic sector is maximal $\left(m_{\tilde{r}} \rightarrow m_{\tilde{p}_{\text {iof }}}\right)$. Indeed this is
- generic feature which we will find common to all processes examined in this paper. (Since we heve asumed that $m_{\lambda}, m_{-j}>m_{\mu}$ in the above expressions, it

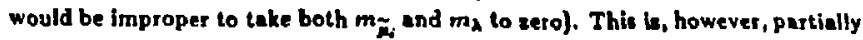
an artifact from dividine (2.9) through by $m_{\lambda}$ to get (2.13). This new contribution depends fundamentally upon the photino tipping helicity, contributing a factor of $m_{1}$ which replaces one factor of $m_{\mu}$, and so vanishes as $m_{2} \rightarrow 0$. As lone as the photino possesses any nnite non-zero value $\left(m_{\lambda} \neq 0\right)$ we maximize $\left|\Delta a_{\mu}\right|$ by taking either $m_{m_{i}}$ or $m_{m}$, 6 be wh ligh a is consistently possible with experiment and the other to be much heavier. Since experimentally $m_{\tilde{p}_{i}} \gtrsim 25$ GeV we might consider the case $m_{\lambda}<m_{n_{1}}<m_{m_{2}}$. In this limit (2.0) becomes

$$
\Delta \Delta_{\mu}=-\frac{\alpha}{4 \pi} \frac{m_{\lambda} m_{\mu}}{m_{h_{1}}^{2}} \operatorname{ain} \phi \cos \phi \text {. }
$$

Although such "large mass-splitting" cases we not ruled out one zenerally Ends, If explicit supersymmetry-breaking terms have their ultimn e orizin vis opontaneous supersymmetry-brekking at some larger mass ucale, that stales tend to have meager mass-aplitting (in this case $\delta m_{\tilde{n}}=m_{\tilde{\mu}}-m_{\tilde{\mu}_{1}}<m_{\tilde{\mu}_{1}}$ ). Indeed onc is led in some models 1 ' to the conelusion that, whould the spontaneous breaking arise in the gauge (" $\left.D^{n}\right)$ terms of the superpotential, then the mass-splitting is expected to be very amall. In such models $\tilde{\mu}_{L}$ and $\tilde{\mu}_{R}$ possess equal masses and are unmixed at the tree level but develop a small mixing term at one loop vis diacrams wech as that in Fiz.2.2.

The result is a smuon mas matrix of the form

$$
\left(\begin{array}{ll}
\tilde{\mu}_{L} & \tilde{\mu}_{R}
\end{array}\right)^{*}\left(\begin{array}{cc}
m^{2} & 6 m^{2} \\
\sigma m^{2} & m^{*}
\end{array}\right)\left(\begin{array}{l}
\tilde{\mu}_{L} \\
\tilde{\mu}_{R}
\end{array}\right)
$$

where

$$
\begin{aligned}
\delta m^{2} & \sim O\left(\frac{a}{\pi}\right) m_{\mu} v \\
m^{2} & \sim O\left(v^{2}\right) \\
v & \sim O(100 \mathrm{GeV})
\end{aligned}
$$


and $\left|m l_{1}-m l_{3}\right|=2 \delta m^{2}<m l_{1,0}$. The mixing angle bo new maximal."

Should the superaymmetry-breaking terms arina in the Yukawn $(" F \overline{)}$ ) eector certain scenarios" euzsest that the $\tilde{\mu}_{L}-\tilde{\mu}_{R}$ mixing engho, $\phi$ would be small although the mans aplitiing could be lasger.

When the musosplitting iz small we find (for $m_{\lambda}<m_{\mu}$ )

$$
\begin{aligned}
& \Delta a_{\mu}=-\frac{s m^{2}}{m_{l}^{2}} \frac{\alpha}{4 \pi} \sin \phi \cos \phi \frac{m_{\lambda} m_{\mu} m_{l}^{2}}{\left(m_{l}^{2}-m_{l}^{2}\right)^{4}} \\
& \times\left\{1+1 \frac{m_{\lambda}^{l}}{m_{l}^{l}}\left[1-\ln \frac{m l}{m l}\right]-\frac{m l}{m l}\left[5+2 \ln \frac{m_{i}^{l}}{m_{l}^{l}}\right]\right\}
\end{aligned}
$$

where

$$
S m^{2}=m_{j_{1}}-m_{j,}^{2} \quad m_{i}=m_{m_{2,0}}
$$

$$
\Delta a_{\mu}=-\frac{\delta m^{2}}{m_{l}} \frac{a}{4 n} \sin \phi \cos \phi \frac{m_{\mu}}{m_{\perp}} N(x)
$$

where

$$
\begin{aligned}
& x=m^{2} / m_{\lambda}^{2} \\
& p(x)=\frac{x}{(x-1)^{4}}\{(x+5)(x-1)-2(2 x+1) \ln x\} \\
& p(x \rightarrow 0) \rightarrow-1 x \ln x \\
& p(x \rightarrow 1) \rightarrow \frac{1}{6} \\
& p(x \rightarrow \infty) \rightarrow \frac{1}{x} .
\end{aligned}
$$

(Nole that this formulation is equivalent to that of referesez a provided that one allows for the difference in the definition of a uned there.)

- Since thin in aclually a two-loop procew there are a umber of other diegransmatic confey. urations of comparsble magaitude which were not iefluded in the oriegal treatment. This ment be dowe if 1 mare precine model is required.

\subsection{COMPARLOH WITH EXPERBLET}

Sooner os later it behooves us 10 make contact with reallty. Here we we the experimental bimits on mesurements of $a_{4}$ and $a_{\mu}$ to plece reatrictions on the contributions considered in the previous subection. It should be noted that ge hes been wo well masured that a signilicane fraction of the uncertainty which is engendered when the empirical and theoretical values ore compared may be as. cribed to the error inherent in high-otder cakulations within the standard model, particulariy from the badronic contributions.

Current limits on the deviation of $\Delta e_{\text {pen }}$ from predicted standard model values are;

$$
\left|\Delta \omega_{\mu}\right| \leq 2 \times 10^{-1}
$$

$$
\left|\Delta c_{1}\right| \$ 3 \times 10^{-10} \text {. }
$$

First tet us consider the implicationn in the case of unmixed aleptons. When $m_{\dot{r}_{L, a}}>m_{\lambda}>m_{\mu}$ we oblsined the result of (2.6) which, wing (2.23), translates to

$$
\frac{1}{m_{k_{2}}}+\frac{1}{m_{n_{n}}^{2}} \leqslant \frac{1}{(10 G+V)^{2}}
$$

This would exclude a circle of the radiu $(10 \mathrm{GcV})^{-1}$ centered at the origin in the $m_{\mu_{L}}^{-1}-m_{\mu_{k}}^{-1}$ plane or, equivalently, the tetion indicated in Fig. 2.3 .

This implies an abrolute limit on the smuon mases of

$$
m_{\pi} \approx 10 \mathrm{GeV}
$$

From (2.5) and (2.21) in the $m_{\dot{e}} \rightarrow m_{\lambda}>m_{e}$ limit we would find a corresponding 
constraint on the selection mess from $\Delta a_{*}$ of unly

$$
m_{\bar{e}} 20.4 \mathrm{GeV}
$$

In passing, we note that should the photlno be substantially more massive then the amuon $\left(m_{\lambda}>m_{F}>m_{\mu}\right)$ then $(2.25)$ would become $m_{\lambda} \gtrsim s \mathrm{GeV}$.

Turning now to the $\tilde{\mu}_{L}-\tilde{\mu}_{R}$ mixed case we will first consider the implicatione of ubbtentlal enen-splitting of the eigenstetes $\tilde{\mu}_{1}$ and $\tilde{p}_{2}$ and then will return to the alightly oplit scentsio. From (2.20) and (2.25) we see that when

$$
m_{\mu}<m_{\lambda}<m_{n_{1}}<m_{n_{\mu}}
$$

that

$$
\left|\Delta a_{\mu}\right|=\frac{a}{4 \pi} \frac{m_{\lambda} m_{\mu}}{m_{j_{1}}^{2}} \sin \phi \cos \phi 52 \times 10^{-1}
$$

or, assuming maximal mixins $(\phi=\pi / 4)$,

$$
m_{\mu_{1}} \geq 39 G e V \sqrt{m_{\lambda}(\text { in GeV })}
$$

We obtain a cimilar limit on $m_{g}$ under weh conditions. In light of the aseumption $m_{\mu_{1}}>m_{\lambda}$ this provides a constraint on $m_{n_{1},}$ for light photina $\left(m_{\lambda}<1 \mathrm{TeV}\right)$. Note that if we let $m_{\lambda}=m_{\mu}$ in (2.27) then the inequality reduces roughly to that of (2.25) in accordance with our observation in the provious eection that one power of photino rass has replaced a factor of lepton mase in $\Delta a_{p}$. This hat its mont profound efiect, of course, when we consider velectrona, as in (2.26), and when the pholino mass is comparable or greater than the mans of the slepton (If $m_{i}=m_{\lambda}$ then both must excetd $0.5 \mathrm{TeV}$ ).
If the mass-splitting is small we might consider the case correspondin to (2.27), i.e.

$$
\frac{J m^{2}}{m_{\mu}^{2}}<1 \quad m_{\mu}<m_{\lambda}<m_{m_{1,1}}
$$

Then from (2.22),

$$
\Delta e_{*} \sim \frac{\delta m^{2}}{m_{\mu}^{2}} \frac{a}{i \pi} \ln \phi \cos \phi \frac{m_{\mu} m_{\lambda}}{m_{\nu}^{3}}
$$

and from (2.23)and (2.24) we obtain

$$
\frac{\delta m^{2}}{m^{2}} \frac{m_{f}+1 \lambda}{m_{1}^{2}} \leqslant 7 \times 10^{-6}
$$

or $\$ 10^{-*}$ in the Eese.

This is not a terribly useful limit. 1, for instence, we were to take $m_{\lambda} \sim 10$ GeV, $m_{\tilde{i}} \sim 100 \mathrm{GeV}$ we would And

$$
\frac{6 m^{3}}{m b} \leqslant O(1)
$$

We note that the above treatment difere only in detail from a full wupers] mmetric elettroweak treatment and the results are vimllar. The principul differcnce in the Intter is thet there are a plethors of contributions from additional guginos and higguinos (which may mix emongut themselves). While we could heve $m_{\tilde{G}}<m_{\tilde{\mu}}$ for a caugino $\tilde{G}$, we might equally well heve $m_{\tilde{g}}>m_{\tilde{j}}$ for a second gautino. The complication in placing bound is evident when we point out that if $m_{\tilde{\mu}} \approx m_{\lambda}$ then (2.20) will becorde (uxins (2.15))

$$
\frac{6 m^{2}}{m_{m}^{2}} S_{4} \times 10^{-3} m_{1}(\text { in GeV })
$$

which is, potentially, significantly more restrictive. We include euch complications when we consider mixing among steplons of different generations. 


\section{FIGURE CAPTIONS}

\section{1. (a) Tran-kval vertex bor a menlan electron.}

(b) One loop QED corraction.

2. Mure-Mixing in the Modal of Ref. 8.

3. Excluded reglon of the $m_{j_{6}}-m_{\lambda_{n}}$ plane if $m_{j_{1}, n}>m_{\lambda}>m_{\mu}$ for caen of no lef-right mixting.

(o)

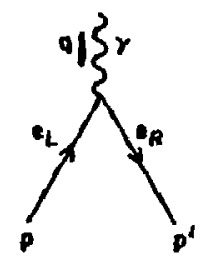

(D)

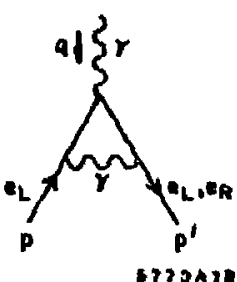

Fig. 2.1 


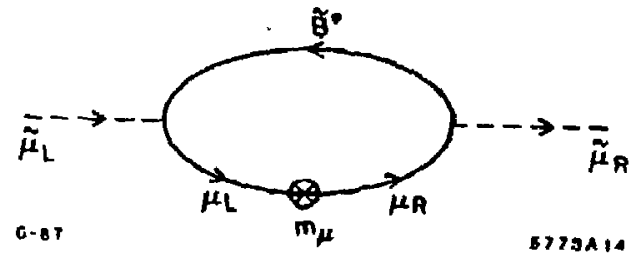

Fig. 2.2

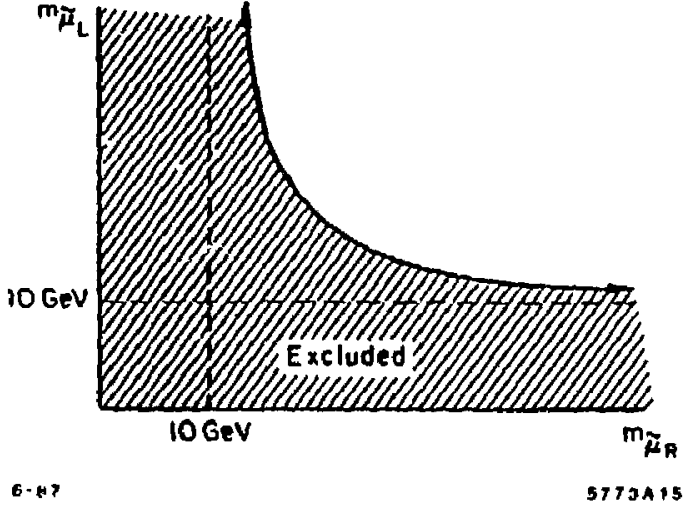

Fig. 2.3 


\section{3. $Z^{0} \rightarrow \mathrm{r \mu}$}

\subsection{JNthoduction}

In this chapter we examine the pousibility of real $z^{0}$ decay in a way which violates lepton family number. We permit the edmixture of the ecaler partners of the keptone (aleptons) from different generation. Thi elaptar duplkate, to a large extent, the worl contained in a previous paper. " We will work in the more familiar four-component Dirae notation. "1H Our notation is based upon that of Refa. 12 and 13. Ueaful eample cakulations uting thin notation may be found in eeveral places. ${ }^{\text {I1,14 }}$

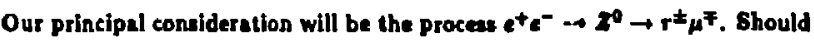
such an interaction accur with a moderate crose-extion it ahould be obenrvable at the emerging generation of colliders. The choke of the find state is womewh arbitrary. It to partially baned on the posibility that the aleplonk masen might prozrea in the same order as their leplonic counterparle and that more manive ates might have a greater tendency to mix. Nelther of these ansumptions in, of coure, necessury, however it is highly desirable to condider thet mot of the mixing oceurn between two cenerations in order to roduce the number or anguler parametern present. There is also the obervation that there are additional box diegrams present if ore of the final states is an electron. When dealine with on-shell $Z^{0}$ such contributione are neglizible and the rasults would be equally applicable to mixing in the $c-\mu$ or $e-r$ sectors. It mut be noted that such computations may be, as will be seen in Chapter 4, readlly extended oftshell.

At in the previous chapter the bulwark of detail will be preaented in related eppendices (Appendices 1-N). Appendix I provides a briel overvlew of ouporoymmetry, motivates the construction of cupersymmetric Legrangia in genera!, 4 tablishes the notation and presente the "atandard superaymmetric Lagranzian". Appendix $J$ considera the dingonalization of the varous mas matrices in different base. In Appendix $\mathrm{K}$ the Feynman tule are derived and detalled examplea of their use given. The computation of the matrix element is done in Appendix $L_{\text {, }}$ notation etabliabed and various integral functions dotined. These are analyzed in detril, both analytically and aumerically, in Appendix M. Finally in Appendix $N$ the vertous background calculationa are presented.

\subsection{THE MODEL}

We consider a minimal eupernymmetric extension of the atandard model and restrkt our at tantion to the leptonic sector. We admit the inclusion ai explicit coft supersymmenry-breaking terms, that is those which do not introduce new quedratic divargence. Thus this "superaymmetric standard model" will devalop arbitrary acalar kpton masus. Praumably all tuch termu arise at come bigher energy wale in a more fundamental way (buch a ouperoymmetry-breaking in a meaner which is sot family-Independent). We shall not concern ouralves here with the ultimale origin of these terme but conelder the efrective low-enercy Lacranglan as givea. In thlo way we may procend in a reasonably model-independent fashion (oubject to the not inubstantial restiction of using pinimal auperaymmetryl.

Given ecalar kptons of arbitrary mas it is only lepton family number, an empirically obeerved tohal (presumebly) symmetry, whicb would prevent thero from mixims. There is some fealing that axact global symmetries are very unnatural ${ }^{(11)}$ (perhape representing the approximate remnents of some more fundamental tocal aymmetry at terger enescy acales). Thus we allow the sleptons to have arbitrary mass and mix with arbitrary anglh. Family-number violation might alto come sbout through any number of other efieets in conjunetion with this or Independently. "(1) We shall not consider this point further other than to note that opecific experimental observations will renarally bo able to distinguiah one model from another (iven auficient statbiks). The same sort of analyais may be applied to the bedronic decay of tbe $Z^{0}$ leading to poustble enhancements of favour symoetry volation in that eeclor. ${ }^{16.17}$ 
We have seen that the left and tight cleptons may readily mix in each of the $\tilde{\varepsilon}$, $\tilde{a}$ and $\tilde{r}$ rectora. W: therelore anticipate mixing amone alx eherged sleptons. In ceneral, mixing armong $N$ atales may be described by $\mid N(N-1)$ real argles and $f(N-1)(N-2)$ complex phase. The latter will typlcally result in CP vlolation. In the above example (three cenerations) we would need 18 real angles and 10 phases. In addition there are three nevtral aneutrino alatea (bix if we permit right-handed sneutrinot in the epectrum) realting in three more angles and one additional phase. In order to reduce the burgeoning number of parametere comewhal we will conslder the case where only the two 'hetrleat' generations mix ignificantly with little left-right mixing. Thus the only mixince are between;

$$
\begin{aligned}
& \bar{\mu}_{L} \text { and } \tilde{f}_{L} \text { with angle } f_{L} \\
& \hat{A}_{n} \text { and } f_{n} \text { with ongle } t_{n}
\end{aligned}
$$

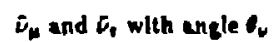

So that

$$
\begin{aligned}
& i_{L_{1}}=\hat{\mu}_{2} \cos \theta_{L}+F_{2} \sin \theta_{2} \\
& \ell_{L_{1}}=-\bar{\mu}_{L} \operatorname{lin} \ell_{L}+\tilde{f}_{L} \cos \ell_{L} \\
& i_{R_{1}}=\dot{\mu}_{R} \cos \theta_{R}+i_{R} \sin \theta_{R} \\
& \ell_{R_{1}}=-\bar{\mu}_{R} \operatorname{tin} \theta_{R}+f_{R} \cos \theta_{R} \\
& \dot{\nu}_{1}=\bar{\nu}_{p} \cos \theta_{p}+\dot{\nu}_{r} \sin \theta_{v} \\
& \dot{\nu}_{2}=-\bar{\nu}_{p} \sin \theta_{v}+\bar{t}_{1} \cos \theta_{v} .
\end{aligned}
$$

with $i_{L, R_{1}}, i_{L, R_{2}}$ and $i_{1,2}$ the physical mass eigenstates.

The particle apectrum of the cuperaymmetric "utanderd" model in lllustrated in Tuble I-1 in Appendix I. The superpotential of the chiral superfieldo must be homogeneous (no terme involving both thiral and antichiral Eelda as one finda in the beld strength) $)^{\mid 1.101}$ with the result that a minimum of two Hiess doublets are required to give the $u$ and $d$ quarks (and thuo charged leptons) masses." In this minimal model we will ignore the possible inclusion of a Higs ainglet, $\mathbf{N}$.
There points are discussed in Appendix I. The fields which will be needed in this chapler are presented in Table 3-1.

A major complication arises in the saugino tector of the theory. Let un ap pose that supersymmetry breaks at an enercy scale above that at which $S U(2) \times$ $U(1)$ does. At large energien we will have exact superaymmetric $S U(2) \times U(1)$. Thus we have masiles $w^{0}, W^{ \pm} S U(2)$ gevge bosons and a massless $B^{0} U(1)$ cauce boson with corresponding sopersyoumetrle parlnera, all massless fermions, $\widetilde{W}^{0}, \widetilde{W}^{ \pm}$and $\tilde{B}^{0}$. Similarly there are two Hize doublete $H_{1}$ and $H_{2}$ with thels cuperpartness $\tilde{\psi}_{H}$, and $\tilde{\psi}_{H}$. Note that the above are Weyl epinors (phis notation differe slightly from that of Ref. 12). It is frequently convenient to write these as lous-componert objects. For the gauginas we shall use the same symbol.

$$
\widetilde{W}^{+}=\left(\begin{array}{c}
-i \widetilde{W} \\
i \vec{W}-
\end{array}\right) \quad \widetilde{W}^{0}=\left(\begin{array}{c}
-i \widetilde{W}^{0} \\
i \widetilde{W}^{0}
\end{array}\right) \quad \tilde{B}^{0}=\left(\begin{array}{c}
-i \tilde{B}^{0} \\
i \tilde{B}
\end{array}\right)
$$

whereas we will diatinguish the Hige four component object as $\tilde{H}_{\text {, }}$

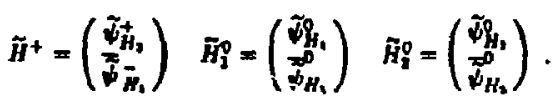

The neutral states are Majorana. Note that $\tilde{H}^{+}$is built from both higesinoe. When supersymmelry breaks, effective low enercy terms of the form

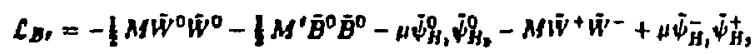

$$
\begin{aligned}
& +m_{i j} \bar{L}_{i} \dot{L}_{j}+m_{i j} \bar{v}_{i} \bar{v}_{j}+\text { h.c. }
\end{aligned}
$$

develop. These are the moat zeneral bilinear soft supercymmetry -breaking terms which may develop. ${ }^{(10)}$ Note that explicit diagonal higssino mass terons are not solt. 
At the weak acale $H_{1}^{0}$ and $H_{?}^{0}$ develop vacuum expectatton values $v_{1}=\left(H_{l}^{0}\right)$ and $v_{2}=\left(\Delta_{2}^{0}\right)$. The atendard electrowenk VEV in replaced by

$$
v=\left(v_{1}^{2}+v_{2}^{2}\right)^{3 / 2} .
$$

The Lerms $f^{i} H_{j}^{0}\left(\ell_{2}\right)_{i}\left(\ell_{R}\right) ;(i=\varepsilon, \mu, \gamma), W^{0} H_{i}^{0} H_{j}^{0}$ and $W^{ \pm} H_{j}^{*} H_{j}^{0}$ of the atendard theory (with two Hiegses) have their supertymmatric colnterparts $f^{i} H_{i}^{0}\left(\tilde{\Omega}_{L}\right)_{i}\left(\tilde{\Omega}_{n}\right)_{i}$, $\widetilde{W}^{0} \tilde{H_{j}} h_{i}^{0}$ and $\widetilde{W}^{ \pm} \widetilde{B}_{i}^{\mp} H_{j}^{0}$ (the general rule is to saplace every pair of talds by their aupetsymmetric partners if $R$ parity is conserved a th here anumed). Now when the neutral higes develop VEVa we devalop more quadratic terme in L, ffurtion. The firat terms give mans to the slepton (this would be equal to the lepton iri=as if it weren't for the edditional term in (3.4)). The othern yleld

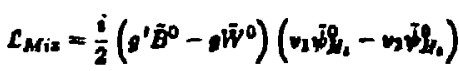

$$
\begin{aligned}
& -\frac{i g}{\sqrt{2}}\left|w_{1} \tilde{W}^{+}+\tilde{v}_{\dot{u}_{1}}+v_{2} \bar{W}-\tilde{w}_{w_{2}}\right|+\text { h.c. }
\end{aligned}
$$

It is convenient to define

$$
\tan 0_{*}=\frac{v_{1}}{v_{3}}
$$

(this is called cot $\beta$ in Rer. 13).

The final term in (3.5) will induce the charged gaugino and higatino atatea of (3.4) to mix. The firat term in (3.5) will cause $\tilde{B}$ and $\widetilde{W}$ to mix with bolh $\tilde{H}_{p}$ and $\tilde{H}_{i}^{p}$. Since $\tilde{H}_{i}^{p}$ and $\tilde{H}_{i}^{0}$ are mixed by (3.5) (by virtue of posessing opposite hypercharges) all four neutral atates mix. We refer to the two churged mass eigenutatee as "charginou" and represent them by $\ddot{x}_{i}^{+}$. The lour neutral mass eigenutates are "neutralinos" represented by $\mathfrak{X}_{\mathfrak{f}}^{0}$. If there are Hizge ainglets then the number of neutralinos will be inereased accordingly. It in also pousible to assign a VEV to the sneutrinos: $\left\langle\tilde{\nu}_{i}\right\rangle \neq 0$. This interesting pousibility loads to further mixing due to terms in $C$ such a $\tilde{4} \tilde{y}^{\circ} \nu$ and bave been examined elsewhere. ${ }^{(m) \mid}$ Such theories explicilly violate $R$-parily, kplon and family number and are therefore of a nomewhat more radical nal yre than will be considered here.
Bince hisesinos and geuginos couple quile differently, the rate of the interaction $e^{+} e^{-} \rightarrow r^{+} \nu^{-}$and simils procoumen will depand upon the detalle of the mass apectrum of the phyaical elgenatele. These effocts may be morte profound away from the $Z^{\circ}$ reconance vince there will be contributions from charginoe and neutralinos in the t-chanuel.

\subsection{MASS EIGENSTATES}

The Lagrangian may be concisely written in terms of chargino and neutralino mase matrices. The unitary matrices which diagonalize the Lagrangian are called $N(a \times 1)$ for the neutralino sector and $U$ and $V(2 \times 3)$ for the cherginos. The detrile are given in Appendix 3 . Sheae matrice will perforce appear in the Feynmen rulve as derived in appendix $K$. The mont involved combinations will occur in the non-Abelian verticas which contain more than one gaugino or biessino. Following Hober and Kan ${ }^{\text {(1) }}$ we represent the chargino combination by matrices $O^{\prime}$ and neutralino by $O^{\prime \prime}$. Since the paramature in the gausino mase matrices may vary over a wide range of parameters one might be wary of finding that one or more alas eigenvalues bave become nogative. This does indeed eppear to oxicur but this does not imply the existence of a tachyon. It asserts en inuppropriate set of basio shites were selected for parameters in this regime. These may be redefined ox, equivalentily, an altered diegonalization procedure used (which accomplishes the sume end). Indeed in the simple $2 \times 2$ chargino system the latter approuch hee seen adopted leading to slight modifications in the Feynman rules as this threshold is brouched. It is also possible, and often desirable, to retain the negative states for computational purposes.

The chargino musea are given by the explicit expressions 


$$
\begin{aligned}
& m_{\tilde{x}_{i}^{*}}=\mid\left(M_{+}+M_{-}\right) \\
& m_{\tilde{x} \tilde{y}}=1\left\{\left(M_{+}-M_{-}\right\}\right. \text {Lign|D| } \\
& M_{ \pm}=\sqrt{(M+\mu)^{2}+2 m_{W}^{2}\left(1 \mp \sin 2 L_{4}\right)^{2}} \\
& D=M_{\mu}-m_{\mu}^{2} \sin 20 \text {, }
\end{aligned}
$$

where $M_{,}, 0_{1}$, are the breaking parameters described in (3.4) and (3.0).

The neutralino masses are given by Ref. 12 a the engenvaluee of the matrix

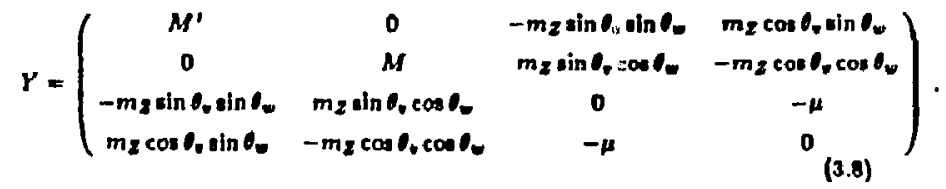

The zeneral solutions are the roots of a quertic equation. This can be golved exactly but proves to be unilluminating. (') Furthermope, If a Higes singlet is added $\boldsymbol{Y}$ becomes $5 \times 5$ matrix and the correspondinis quintic root equation will have no closed form solution in general. ${ }^{\text {(1) }}$ Note thall in apecific casea, such os those discussed in sppendix $\mathrm{J}$, nitnplifed forms are fre gyuently posulble.

Mass toatrices for the sleptons may be similarly constiructed. There are ondiagonal contributions from the lepton mass and the explicit breaking terms. The off-diagonal entries derive solely from the soft terms. Since lepton masses are expected to be much smaller than the superaymmetry-breaking terms they may -llectively be iznored except in special circumstances (wch as lifhing a degeneracy or preventing the matrix from becorain singular). Since all of the entsies are then "arbitrary", it is suficient to merely consider the phywical eigenstates and ehorocterize them by siven mixing angles end masses whout reference to the unmixed precursor states.

\subsection{Matalx Elements}

The diagrams contributing to $Z \rightarrow \mu r$ are fou $\rightarrow$ d in Fig. 1 . Details may be found in Appendix $\mathbf{N}$ using the Feynman rules derived in the previous appendix. The explicil expression for the lexding order term in the matrix element, $M^{*}$. is given by 
$\mu^{\omega}=M_{L} \varepsilon_{L}+M_{A} \varepsilon^{\omega}$

$\epsilon_{t, R}=\tilde{E}^{\mu}\left(p_{1}^{\prime}\right) \gamma_{\mp} \gamma^{N} \gamma_{ \pm} \nu^{\prime}\left(p_{2}^{\prime}\right) \quad \gamma_{ \pm}=f\left(1 \pm \gamma_{1}\right)$

(3.0a)

$$
M_{L}=\frac{K_{\nu}}{\sin ^{2} \omega_{\omega} \sin 2 U_{\omega}} \sum_{i=1}^{t}\left\{\left|V_{i 1}\right|^{2}\left[\Delta G\left(T_{i}^{+}, R_{i}^{+}\right)+\cos 2 \omega_{\omega} \Delta G\left(T_{i}^{+}, 0\right)\right]\right.
$$$$
+2 \sum_{j=1}^{2} V_{i i} V_{j 1}\left[\sqrt{s_{i j}^{+}} o_{i j}^{R^{\prime}} \Delta J_{[1]}\left(T_{i}^{+}, s_{i j}^{+}, R_{i}^{+}\right)\right.
$$$$
-R_{i}^{+} O_{k j}^{C^{\prime} \Delta} \Delta I_{[-2-2+1}\left(T_{i}^{+}, S_{i j}^{+}, R_{i}^{+}\right)
$$$$
\left.+O_{i j}^{t^{\prime}} \Delta \sigma^{*}\left(T_{i}^{+}, s_{i j}^{+}, R_{i}^{+}\right)\right\}
$$

$-\frac{K_{L}}{2 \sin ^{2} \theta_{w} \sin 2 \theta_{w}} \sum_{i=1}^{l}\left\{i \operatorname{sen} \theta_{\omega} N_{i 1}+\left.N_{i 1}\right|^{2} \cos 2 \theta_{\omega} \Delta \tilde{G}\left(T_{L}^{0} i, R_{i}^{q}\right)\right.$

$-2 \sum_{j=1}^{1}\left(\operatorname{lan} \omega_{\infty} N_{i i}+N_{i j}\right)\left(\tan \theta_{-} N_{j 1}+N_{j 3}\right)$

$\left[\sqrt{S_{i j}^{0}} o^{R_{i j}^{\prime \prime}} \Delta J_{\mid 2]}\left(T_{t}^{0}{ }_{i,}, S_{i j}^{0}, R_{i}^{0}\right)\right.$

$$
-R_{i}^{0} O_{i j}^{\prime \prime} \Delta I_{[\tilde{E}=-2)}\left(T_{L}^{0}{ }_{i,} S_{i j}^{0}, F_{f}^{q}\right)
$$$$
\left.\left.+O^{L}{ }_{i j}^{\prime \prime} \Delta G\left(T_{L}^{0}{ }_{i}, S_{i j,}^{0} R_{i}^{0}\right)\right]\right\}
$$

$$
M_{R}=\frac{1 K_{R}}{\sin ^{2} \theta_{w} \sin 2 \theta_{\omega}} \sum_{i=1}^{\infty}\left\{\left|\operatorname{Lan} \theta_{\omega} N_{i j}\right|^{2} \sin ^{2} \theta_{\omega} \Delta \ddot{G}\left(T_{R}^{0}{ }_{i}, R_{i}^{0}\right)\right.
$$

$+\sum_{j=1}^{i}\left(\tan \theta_{w} N_{i 1}\right)\left(\ln \theta_{\infty} N_{j i}\right)$

$\left[\sqrt{S_{i j}^{0}} o^{L \prime \prime} \Delta I_{|1|}\left(T_{x}^{0}{ }_{i,} S_{i j}^{0}, R_{i j}^{0}\right)\right.$

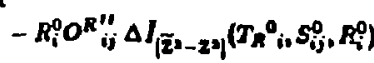

$\left.\left.+O^{R}{ }_{i j}^{\prime \prime} \Delta G\left(T_{R}^{0}{ }_{i,} S_{i j}^{0}, R_{i}^{0}\right)\right]\right\}$

$$
\begin{aligned}
& K_{v}=\frac{i}{16 \pi^{2}} e^{2} \sin \theta_{v} \cos \theta_{v} \\
& K_{L}=\frac{i}{16 \pi^{2}} e^{2} \sin \theta_{L} \cos \theta_{L} \\
& K_{R}=\frac{i}{16 x^{2}} e^{2} \sin \theta_{R} \cos \theta_{R}
\end{aligned}
$$

\section{$R, S$, and $T$ are ration of manew:}

$$
\begin{aligned}
& T_{i}^{+}=\frac{m_{i_{i}}^{2}}{m_{\bar{x}_{i}^{2}}^{2}} \quad T_{L,} i_{i}^{0}=\frac{m_{i_{L, i}}^{2}}{m_{\dot{x}_{i}}^{2}} \\
& s_{i j}^{+}=\frac{m_{\bar{x}_{i}^{2}}^{2}}{m_{\hat{x}_{i}^{2}}^{2}} \quad s_{i j}^{0}=\frac{m_{\hat{x}_{i}^{2}}}{m_{\bar{x}_{i}^{2}}^{2}} \\
& R_{i}^{+}=\frac{e^{2}}{4 m_{x_{i}^{+}}{ }^{2}} \quad R_{i}^{0}=-\frac{q^{2}}{4 m_{x_{i}}^{2}}
\end{aligned}
$$

where $m_{\tau_{L_{2}}}$ and $m_{\tau_{s_{1}}}$ are the masses of the charged slapton of the firat peneration mixture (cee (3.1)) and $m_{\nu_{1}}$ b the mes of the and innoutrino $\left(\tilde{\nu}_{1}=\tilde{\nu}_{\mu} \cos \theta_{\nu}+\right.$ $\left.\tilde{v}_{v} \sin \theta_{v}\right)$. The aeutralino mases are $m_{\bar{x}} \quad(i=1,2,3,1)$ and chargino mases we $m_{\tilde{x}_{i}^{*}} \quad(i=1,2)$. The enercy of the $2^{0}$ is $q^{2}=m_{q}^{2}$ since we are working an-shell. The integral functions $\left(\Delta I_{|N|}, \Delta G, \Delta G, \angle, \bar{b}\right)$ are defined in Appendix $N$ and analyzed, andytically and numericatly, in the subsequent appendix. The matrix element $M^{*}$ cennot be exemined in all of its complexity (there are, after all, $120 \mathrm{kerm}$ in Eq. (3.0)) but there are a number of apecial casen which prove instructive. These principally involve taking the breaking parameters to extreme values (zero or infinity) and a number of examples have been examined. We find, In accordance with the decoupling theorem, ${ }^{\text {pH }}$ that as the physical mass of a matrix becomes large its contributions to the cross-section become progressively 
less Ioportent, finally vanihing. The matrix element remains finite for all values of the parameters involved."

\subsection{PRODUCTION THRESHHOLD}

If any of the supersymmetric particles is sufficlently light $\left(m<\mid m_{2}\right)$ then real production occurs. I such stales are unatable (zenerally only the least massive cupereymmetric atate will be slable, and only if $\boldsymbol{R}$ b conserved) then final state processe with four or more particle will dominale. If the lightest eupersymmetrle particle la neutral then all such decays are characterized by "missing mas" in the final atate. Such aignals are notoriously amenable to silernate interpretations yet remain the hallmark of experimental supersymmetric aesches. Below threbhold there are atill contributiono to closed loop processes, wuch as the one under consideration, however the matrix elementa become complex.

Let un asume that a nearly mussless eneutrino wete the llahleal eupersymmetric particle and, furthetmore, was alable. The reaction $e^{+} e^{-} \rightarrow z^{0} \rightarrow$ iv would appens as $\mathrm{c}^{+} \mathrm{e}^{-} \rightarrow$ nothing. The real particle produstion analogues of the processes In Fig. 3.1 would be

$$
\begin{aligned}
& e^{+} e^{-} \rightarrow z^{0} \rightarrow \tilde{x}^{-} \tilde{x}^{+}
\end{aligned}
$$

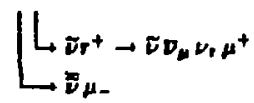

and

$$
\begin{aligned}
& e^{+} e^{-} \rightarrow Z^{0} \rightarrow t^{\pi} t^{-}
\end{aligned}
$$

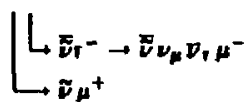

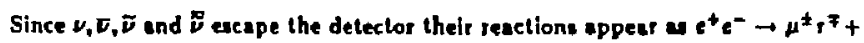

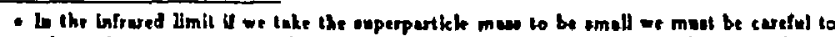

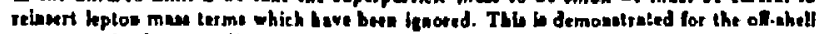
are vertex in the eppendices asoocinted with Chaptor 4.
}

missing energy. This dix-particle fnal state would be difficult to distinguish from far more quotidian oceurrences such as $e^{+} e^{-} \rightarrow r^{+} r^{-} \rightarrow \mu^{+} \mu^{-} \nu_{\mu} \nu_{1} \nu_{1} \nu_{\mu}$.

\subsection{Small Mass Splittings}

The lasgest cross-sections okcur when the must-splitting between $Z_{L, n_{1}}\left(\bar{v}_{1}\right)$ and $i_{L, A_{1}}\left(\bar{V}_{2}\right)$ is lerce. In many models we may restrict ourselves to the case where the relative mass-splitting is small, i.e.

$$
\delta_{i_{2}}<1, \quad \phi_{i}<1, \quad \sigma_{i}<1
$$

where

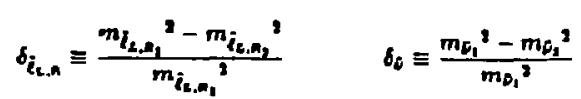

These models ${ }^{[2]}$ ussume (quile ressonably) that superiymmetry breakn spontaneously in a flevour-independent mannes. I the breating scale in not too larce then simall mass aplitting followe naturally. This has not been asumed in this paper. In tuch ccenarios the matrix elaments are conerally of least an order of magnitude omaller. In this limit we can write

$$
\begin{gathered}
\Delta F\left(T_{i}^{+}, S_{i j}^{+}, R_{i}^{+}\right)=\delta_{i} F^{\prime}\left(T_{i}^{+}, S_{i j}^{+}, R_{i}^{+}\right) \\
\Delta F\left(T_{L, R_{i}}^{0}, S_{i j}^{0}, R_{i}^{0}\right)=\delta_{i_{L}, n} F^{\prime}\left(T_{L,} R_{i}^{0}, S_{i j}^{0}, R_{i}^{0}\right) \\
\Delta F \equiv F\left(i_{1}\right)-F\left(\bar{l}_{2}\right) \doteq \delta_{i} F^{\prime}\left(\bar{l}_{1}\right) \\
F^{\prime}(T, S, R) \equiv T \frac{\partial F(T, S, R)}{\partial T}=\frac{\partial F(T, S, R)}{\partial \ln T}
\end{gathered}
$$

In this case we may seplace each $\Delta$ ) and $\Delta G$ term in (3.0) by $b_{i, b} J^{\prime}$ and $\delta_{i, c} G^{\prime}$. (See Appendix $\mathbf{N}$ for these functional forms). 


\subsection{Cross-section and spegial Cases}

Given the matrix element, $\mathcal{M}^{\circ}$, in (3.0) we may darive

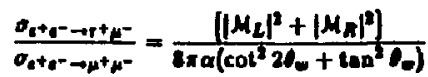

Note that if any of the auperzymmetrie particles has a mass of lass than $\mid M_{3}$ that real production can occur and thet $\mu_{L}$ and $\mu_{R}$ are complex. It is the modulus which in important in (3.16).

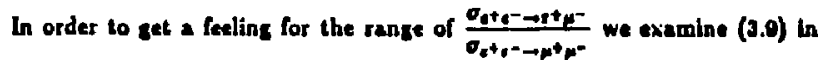
two extreme caugino linits. The first to the "uuperaymmetry" limit in which we eliminate the gaugino supersymmetry-breaking terme by ketting $M^{\prime}, M, \mu \rightarrow 0$ and let $v_{1}=v_{2}$. Thus the only superaymmetry-breakins term are the explicit slepton mass terms. Then (3.9) reduces to

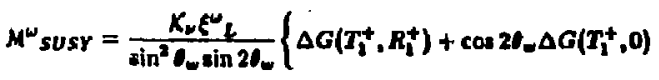

$$
\begin{aligned}
& -\cos 2 \omega_{\omega} \Delta J_{[1}\left(T_{1}^{+}, R_{1}^{+}\right) \\
& \left.+2 \cos ^{2} \theta_{\omega}\left[R_{i}^{+} \Delta I_{\left(\tilde{z}_{2}-2, j\right.}\left(T_{1}^{+}, R_{1}^{+}\right)-\Delta G\left(T_{i}^{+}, R_{1}^{+}\right)\right]\right\} \\
& -2 K_{L} \epsilon_{L}{ }_{L} \cot 20_{\omega}\left\{\Delta \bar{G}\left(T_{L}{ }^{0}{ }_{1}, R_{1}^{0}\right)+\cot ^{2} 20_{\omega} \Delta \dot{G}\left(T_{L}^{0}{ }_{2}, R_{L}^{0}\right)\right\} \\
& +2 K_{R} \epsilon_{R} \tan \rho_{\omega}\left\{\Delta \bar{G}\left(T_{R}{ }^{0}, R_{1}^{0}\right)+\tan ^{2} \theta_{w} \Delta \bar{G}\left(T_{R}^{0}{ }_{2}, R_{2}^{0}\right)\right\}
\end{aligned}
$$

with

$$
\Delta I_{[\mathrm{N}(z, Z)]}(T, R)=\Delta I_{[\mathrm{N}(Z, z)]}(T, S \equiv 1, R) \quad G(T, R)=\Delta G(T, S \equiv 1, R)
$$

where the physical masses are now

$$
m_{\dot{x}_{i}^{+}}=\left(M_{w}, M_{w}\right) \quad m_{\bar{x}_{i}^{*}}=\left(0, M_{z}, M_{z}, 0\right)
$$

and the relovant mane retios are

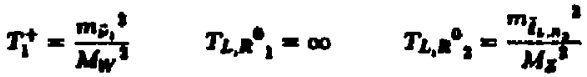

$$
\begin{aligned}
& R_{1}^{+}=\frac{1}{4 \cos ^{2} \theta_{\infty}} \quad R_{1}^{0}=\infty \quad R_{i}^{0}=1 / \kappa
\end{aligned}
$$

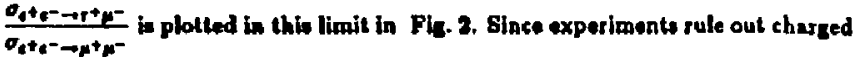
appernymmetry partaers of mase bes than sbout $24 \mathrm{GoV},{ }^{\mathrm{BH}}$ and in some inatence much stronger limits have been pleced. $18 \mathrm{P}^{(3 / 4}$ he pleced limits on the eelectron maes and the wino mas of approximetely $00 \mathrm{GoV}$ Whon combined with the tesulte of other groups they find that $m_{i} \geq 84 \mathrm{GoV}$. While ouch strong limits do aot, as yet, exist for $m_{j}$ or $m_{m}$, and hence for $m_{i_{1,}}$, it would be somewhat eurpriaing to find $m_{j}<m_{j}$. We see thet for light elegton masee with lere mas-

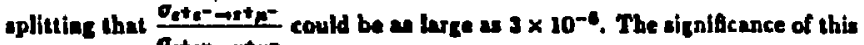
will be diecuned shortly. In order to echitve such a value parameters conducive to a lurge cross-section have been selected. Wo have allowed the sleptons to mix

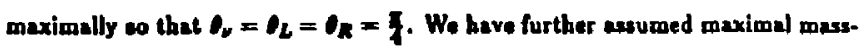
eplitting between the two elepton exclors, i.e. $m_{\sigma_{1}}, m_{i_{L_{1}}}$ and $m_{i_{n_{1}}}$ are relatively light but $m_{i_{3}}, m_{i_{b}}$ and $m_{j_{a}}$, are large and decouple.

Another extreme limil in the "unmixed" limit in which the Higesino and Gaugino sectors have been dientanglad from one another. There are aeveral ways of echjeving this. Here we consider the limiting case $M \rightarrow \infty, M^{\prime} \rightarrow 0$ and $\mu \rightarrow 0$ (0. arbitrary). The physicnl mass states become

$$
M_{\bar{x}_{i}^{+}}=(M \rightarrow \infty, 0) \quad M_{\hat{x}]}=(M \rightarrow \infty, 0,0,0)
$$

Note that $\bar{x}_{1}^{0}=\overline{\boldsymbol{F}}_{3}^{0}, \bar{x}_{3}^{0}=\tilde{B}^{0}$ and thus are purely cautino wheress $\dot{x}_{3,4}^{0}$ are

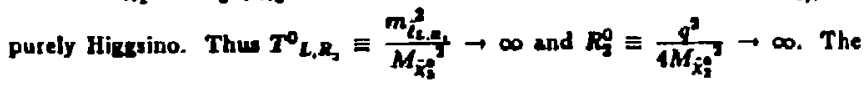


watrix element o given by

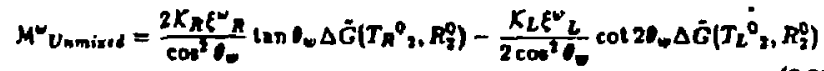

Note that this result in independent of $\boldsymbol{O}_{6}$. The correspondine cross-rection is plotled in Fis. 3. Again the sleptom are assumed to have maximal mixint and wass-splittint. Note, however, that in this limiting case we have a massless chargino (s eharted Hicasino). Since experimentally the mass limit for new charged particles is $-21 \mathrm{GeV}$, we conclude thet this case fo merely fllustrative.

\subsection{Expermiental Considenations}

The question arises as to whether ouch a process would be obeervable at SLC or LEP. These machines will operate $a Z$-factories and wo se ideally suiled for such s search. We aee from Fig. 2 that, at best, the erousesection for $e^{+} e^{-} \rightarrow$ $Z^{0} \rightarrow r^{+} \mu^{-}$will be $\sim 3 \times 10^{-6}$ that of $e^{+} e^{-} \rightarrow Z^{0} \rightarrow \mu^{+} \mu^{-}$. Thus we expect

$$
B R\left(2^{0} \rightarrow 1^{+} \mu^{-}\right) \leq O\left(10^{-7}\right)
$$

where we have used the relevent standard branching ration

$$
B R\left(Z^{0} \rightarrow \mathrm{r}\right)=B R\left(Z^{0} \rightarrow \Delta \mu\right)=B R\left(Z^{0} \rightarrow C e\right) \simeq 3 \%
$$

We also note that

$$
B R(r \rightarrow \mu D \nu) \simeq B R(r \rightarrow C D \nu)=17.5 \%
$$

The experimental signature for auch $\&$ decay would be $\mu$ and $r$ back-to-back with the $\mu$ having $E=\frac{M_{2}}{2}$ and no miasing energy. The principal bectground will be from $e^{+} e^{-} \rightarrow Z^{0} \rightarrow r^{+} r^{-}$followed by $r^{ \pm} \rightarrow \mu^{ \pm} D \nu$, with the $\mu$ heving nearly all of the $\mathrm{r}$ momentum. The $\mathrm{r}$ will travel approximately $2.8 \mathrm{~mm}$ before decaying in this case and, since the $r$ and $\mu$ ere nearly collinear, the kink in the track will be unobservable. Clearly the number of $\mu, N(\Delta \varepsilon)$, produced in the enercy ranze from $\frac{M_{2}}{2}-\Delta c$ to $\frac{M_{2}}{2}$ in of paramount Importance. We find that (see Appendix N)

$$
N(\Delta \varepsilon)=\frac{3-a}{9+a} \cdot 24 \frac{\Delta \varepsilon^{2}}{M !}
$$

where a mensures the degree of polarization ( $a=0$ for the unpolarized case; $a=1$ for complele polariention). At SLAC we expect $a \approx 1$ and thus $N_{\text {wol }}(\Delta c) \doteq \frac{21}{5} \frac{\Delta f^{2}}{M I_{2}}$. From this and $(10 \mathrm{~b})$ we see that we need $\Delta \varepsilon \leqslant 0.1 \%\left(\frac{M_{2}}{2}\right)$ to achieve a background of $10^{-7}$ or less which would compare with (3.22). Thus for muons with $E \approx \frac{M z}{2}$ we need $\frac{\Delta p}{p} \approx 0.1 \%$.

At the SLC the MARK II detector will have an enercy resolution for e's and $\mu$ ' of $\sim 0.3 \% / G e V$ without a vertex delector and, perhaps, w low a $0.1 \% / G e V$ with the planned vertex deteclor. ${ }^{\text {(2) }}$ For $\frac{M z}{z}=50 \mathrm{GeV}$ this means that $\frac{\Delta_{P}}{p} \sim 5 \%$ and from (3.25) the background will uwamp the signal by at least a factor of 200. It eppears unlikely that delector momenturn resolutions will be improyed by the required two orders of magnitude in the neor future (there remains the formidable problem of increased multiple acalterings as detector mass in added). Thus the procese $e^{+} e^{-} \rightarrow Z^{0} \rightarrow+^{+} \mu^{-}$will not be experimentally observable at the emerging getnetation of machinet. Furthermore, because of (3.22), when SLC achieves its eventual target luminomity of $10^{\circ} Z^{\circ} /$ year, the $Z^{0} \rightarrow+^{ \pm} \mu^{*}$ production rale will be at beal $\sim 0.2$ event/year.

Should the mixing be primarily between $\bar{z}$ and $\bar{\mu}$ there will be an important additional restriction to consider. Under the oume conditions deacribed above (maximal gnixing; latge mass-splitting; $Z^{\circ}$ on thell) the production rate would uppear to be the ame. It is lound, however, that the experimental timits on the process $\mu \rightarrow C$ place severe constrainto on $\bar{z}-\bar{\beta}$ mixing in the neutralino pector. If we assurne maximal mixing and large mast splitting then (chapler 1) we Bnd that the lightent alepton has $m \geq 1 \mathrm{TeV}$ and 10 decouples. Thus the 
charginos provide the sole contribution to $Z \rightarrow \mu^{+} e^{-}$(other than in exceptional eircumstances). The matrix element it then proportional to the eneutrino mass splitting. Asuming that this is aleo hrge we find, for cases of interest, that the chargino sector contribution is generally much lerger than that of the nevtralino sector even before the above constraint has been imposed. Thus the removal of the reutralino contribution, while considerably ilmplifyinis calculations, would not greatly affect the final cross-section.

For the aituation illustrated in Fir. $3(b)$, when applied to $Z \rightarrow \mu^{+} c^{-}$, we find that imposing the above constraints on $m_{i}$ affect the realts by, at most, $16 \%$. Furthermore, in the region of intereat, this change results in an increase in the cross-section (because the real parts of the neutralino and chargino contributions enter with opposite signs). Note that the preceding argument (except the quoted percentage) also follown in the cases of amall maswaplitting and non-maximal mixing.

When considering the proces $Z^{0} \rightarrow r^{+} \mu^{-}$eurrent limits from $r \rightarrow \mu \gamma^{(2)}$ do not impose serious constraints on the neutralino sector. In the case of large mass-splitting and maximal mixing we find only that $m_{i} \geq 13 \mathrm{GeV}$. In particular substantial effects pertist in thit cate (arising from the neutralino tector) even when $m_{\dot{v}_{1}}=m_{\xi_{2}}$.

We can do somewhat better on background if we assume that the princlpal slepton mixing occurs in the $\dot{\boldsymbol{c}}-\dot{\boldsymbol{\mu}}$ sector. The principal backeround will still

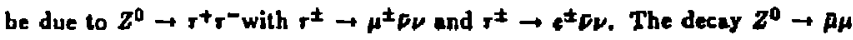
will not be a problem since, at these energies, muons will not decay in the detector. Now misidentification of $a{r^{+}}^{+}$pair $a$ a back-to-back $\mu^{ \pm} \epsilon^{\mp}$ requirea that both the $\mu$ and $e$ emerge with energies near $\frac{M_{2}}{2}$. Awuming complete polarization we find that $N(\Delta p)$, the number of back-to-back $e-\mu$ pairs emerging with $E=\frac{M z}{2}$, to within the experimental momentum resolution $\Delta p$, is given by $N(\Delta p)=1.4\left(\frac{\Delta p}{P}\right)^{4}$. Thus with $\frac{\Delta p}{P} \sim 5 \%$ we find $N(\Delta p) \approx 9 \times 10^{-6}$, which corresponds to roughly 0.02 misidentified events per year, ssuming a sample of $10^{\circ} 2^{\circ}$. It would appenr that, under the moel opportune of conditions, the aignal might stand well above the hackground. It mult be cautloned, however, that such propitious circumatances are singularly unhkely. The actual $e^{+} e^{-} \rightarrow \mu^{*} e^{\mp}$ production rate is almost assuredly much lea than the near-maximal value which we beve hetn considering and thi idealized background calculation has omitted a number of importent experimental effects. This result b, none the less, encouraging particularly in light of the observation that statistice and detector nensitivities will only improve with time.

\subsection{Conclusion}

In conclusion, even in the most favourablescenarion, it appeare unlikely that $\mathrm{C}^{+} \mathrm{e}^{-} \rightarrow \mathrm{r}^{+} \boldsymbol{\mu}^{-}$will be obuerved at SLC within the frat few years of operation if the sole contribution is from slepton family mixing. The production rate is simply inauficient and the buckground rate overly aevere. The decay $\mathrm{C}^{+} \mathrm{C}^{-} \rightarrow \mathrm{H}^{+} \mathrm{e}^{-}$ is equaliy rare but the backzround problems may prove more tractable. The full parameser space of the gaugino-bigzeino sector has yat to be explored. Since the crose-axctions predicted in the limiting cases presented come within an order of magnitude of being experimentally intereating at the SLC, realiatic symmetrybreaking parameters might exist which would increase the cross-section to observable levels. In any instance the projected luminosity available at: LEP might sender such searches feasible. 
Table 3.1 Fields in 'This Chapler

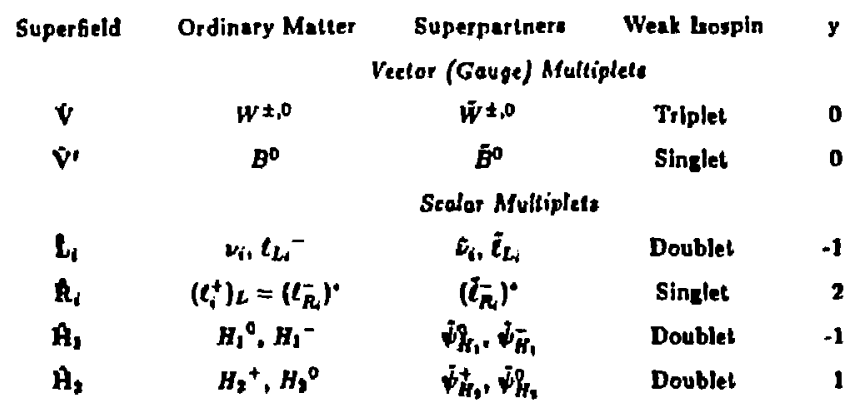

\section{FIGURE CAPTIONS}

1. Disgrams contributing to $2^{0} \rightarrow r^{+} \mu^{-}$.

a) Chargino Diazrams.

b) Neutralino Diagram.

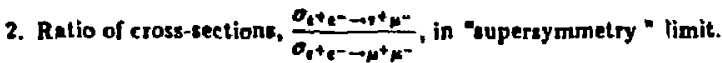
$M=M^{\prime}=\mu=0 . \quad N_{1}=\pi / 4$.

(a) Varying all siepton masses equally. [Jenoring $\mathrm{th}_{i}$, and $m_{F}$ ].

(b) Varyine $m_{t_{L}}$, while holdins all other mass parameters equal to the Indicated values.

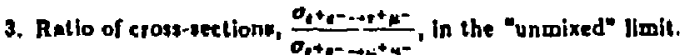
$M=M^{\prime}=\mu=0 . \quad 0_{0}=-/ 4$.

Vay $m_{i_{c_{2}}}=m_{i_{n_{1}}}$ ssuming $m_{i_{L_{2}}}=m_{i_{n_{1}}}=\infty$. 

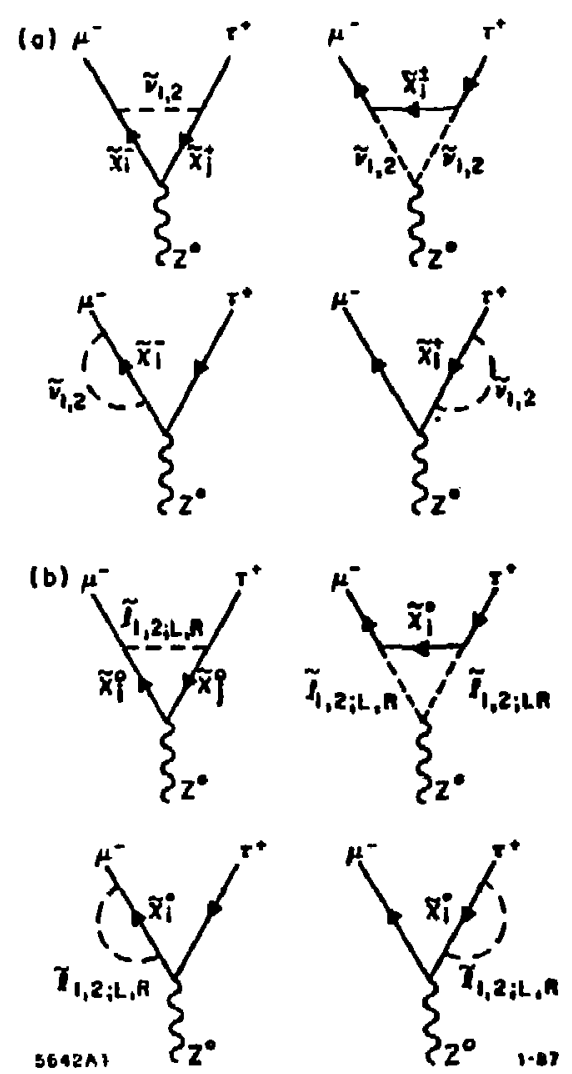

Fig. 3.1
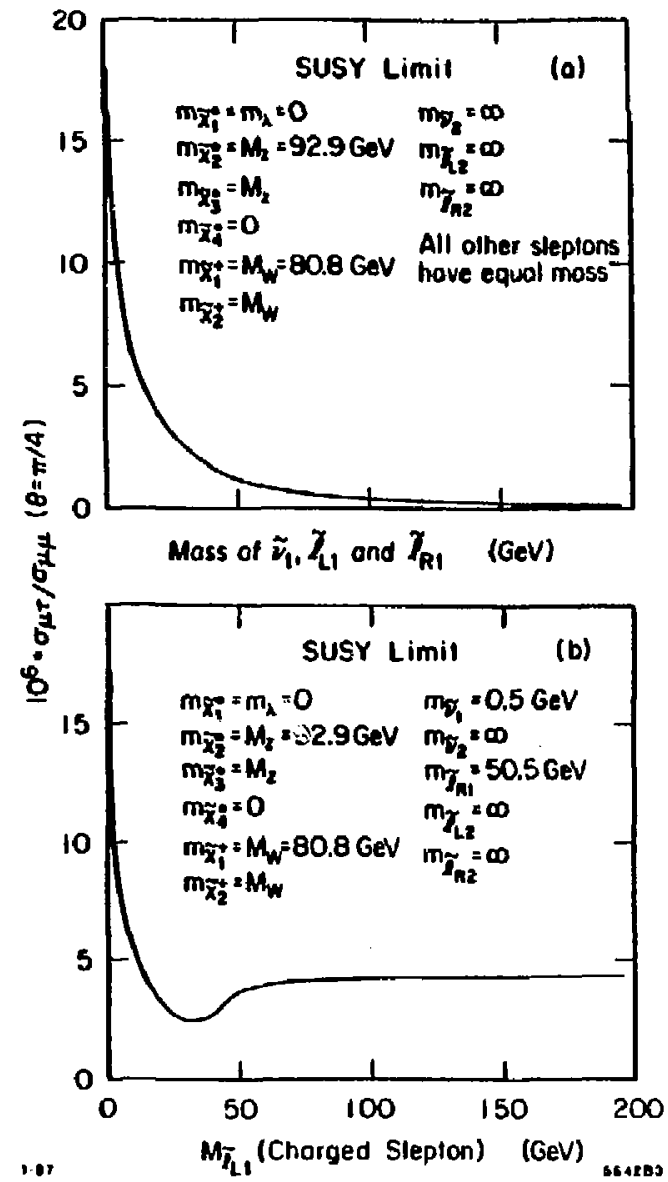

Fig. 3.2 


$$
\text { 4. } \mu \rightarrow \text { er }
$$

\subsection{INTRODUCTION}

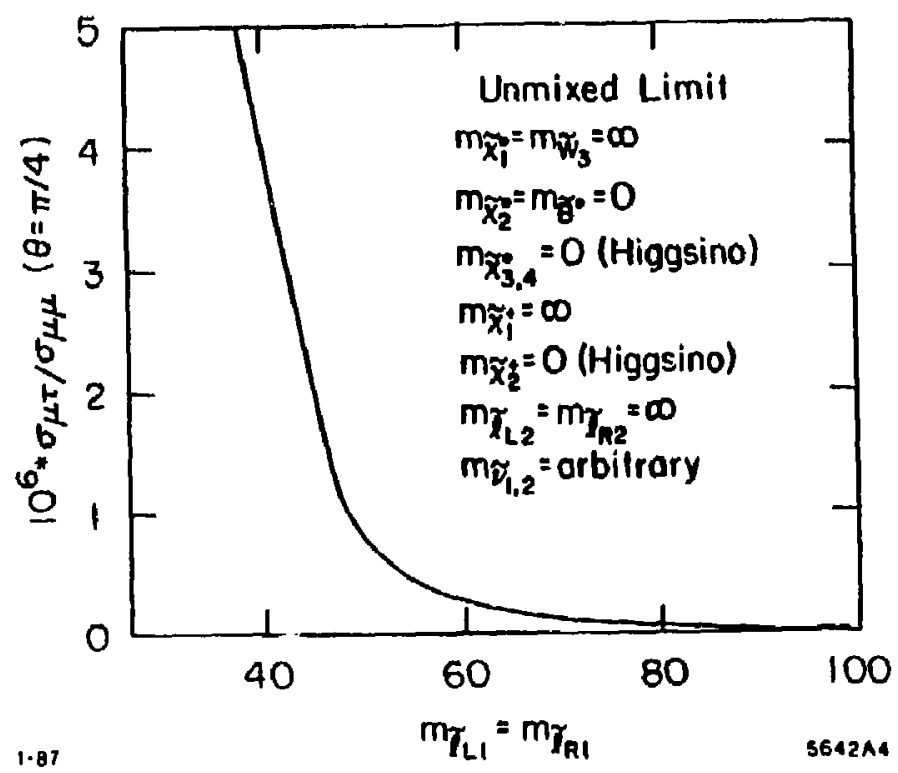

Fig. 3.3
This is the classic tep in faraily number violating proceas. Of nuch procesese it is the best-studied, boti theoretically and experimentally, and that which has provided the keeneat limitc cn model-building. In torm of the pretent model auch - decay would occur due so mixing in the refectronk and stouonic mectors. We could molivate the form of the matrix elements by arat conaldering the related decay $r \rightarrow$ Hy in whleh the mixing brincipally between $\bar{p}$ and $f$ (and the corresponding ectur neut:inot). We would then utllize the wisdon cleaned in the previous chopter by developlng cronaine relatione 10 go from $z^{0} \rightarrow r^{+} \mu^{-}$- 10 $r \rightarrow \mu \gamma$ directly. This would give us the leading ordar vectorlal $\left(\gamma^{\mu}\right)$ Interaction term which con be ohown to vanish due to the resone discunaed in sectlon $\mathbf{4 . 2}$.

The lowett-order non-t:jvial matrix elewents will be presenled (Appendix O) and discussed (in section 4.3). We will consider oftahell behavlour and observe that the "vectorial" terms nay indeed prove important in thl cane. We will not isnore the onsses of the fermions in thls case alnce, unlike the previous chapter, the lepton wasses ase comr arable to the decey enerty. Finally, comparison with experiment will be examin $\mathrm{y}$ in wection 4.4 and apecial cases of Interest presented.

\subsection{THE FORM OF TUE MATRD ELEMENT}

The first observation which we make is that, in the approxisoation of masulen leptons (particularly muons and electrons), $\mu \rightarrow$ e $\gamma$ mut be principally mediated by a convective $\sigma^{\text {we }}$ interaction. The reason is that the photon is masaless and thetefore transverse. Thus it has apin quantum numbers (on thell)

$$
\left(3, s_{3}\right)=(1, \pm 1)
$$

but no $A_{3}=0$ longitudinal part. Hence, denotins the 'z-component' of the spin of the (anti)muon by is ( $B)$, tet us contider the reluted procens of $\gamma \rightarrow$ pe depicted 
in Fig. 4.1. Sinee $d_{3}(\eta)=s_{s}(p)+e_{s}(e)$ and the hptons emerge buck-to-back thay must heve uplns aligned in the wame directlon and so have opposite chiralition. In the weak bast $W^{ \pm 0}$ only couple to helt-hended particle (and likewien for their supersymmetric partners) and wo only bे $^{0}$ would contribute in the fndicated fashion. This conclusion is true if Fig, 4.1 were rotated to fllustrate $\mu \rightarrow \mathrm{er}$. Or course, wince both $\tilde{2}^{\circ}$ and $\tilde{7}$ contain a piece of fo $^{\circ}$ they would both contribule to such a procese. Hiseainos couple with a etrength which is proportiond to the mass of the leptor and wo they aleo do nol contribute. In an arbitrary neutralino

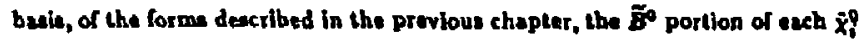
would contrlbute.

When electron and muon masee are included thon belicity tippins becomea poasible along the kpton line and $\widetilde{W} \neq 0, \tilde{H} \pm 0$ diagram will contributa (uop-

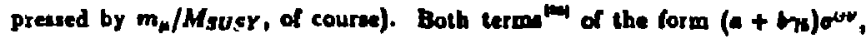
which aip helicity, and $(e+d \eta) \sim$, which do not, are poealble. Ar the helicitypreserving terma are downe by $m_{d} / M_{i w}$ mont papers olmply innore them and asume an interaction of the form obve. Since

$$
\begin{aligned}
& \bar{\psi} \psi=\psi_{k} \psi_{L}+\psi_{i} \psi_{n}
\end{aligned}
$$

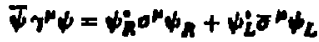

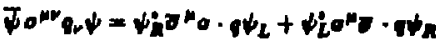

we can rotate $\psi_{2}^{(i)}$ (i is a generation index) and $\psi_{x}^{(j)}$ independently when there are

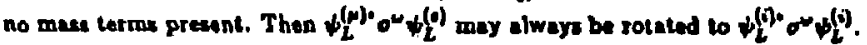
This informs us that the coefficiente of the or terms must always be proportional to sozne power of the lepton masies.

We will use the somewhat non-alndard notation for the pure SQRD case:

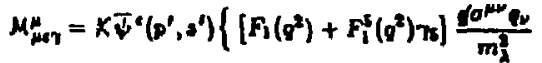

$$
\begin{aligned}
& \left.+\left[F_{1}\left(q^{2}\right)+F_{2}^{5}\left(q^{2}\right) q_{s}\right] \sigma^{\mu \nu v} q_{\nu}\right\} \psi^{\mu \nu}(p, a)
\end{aligned}
$$

where $K$ is a conatant which can be shown to be equal to

$$
K=-\frac{a^{3 / 1} \operatorname{ain} \theta \cos \theta}{111 \sqrt{\pi}}
$$

and

$$
\omega=\boldsymbol{m}-p_{\omega}^{\prime}
$$

in this instance.

The $\tilde{e}-\bar{\mu}$ mixing angle is $\theta$ (here we are tecitly taking $\boldsymbol{\nu}_{L}=\boldsymbol{\theta}_{R}=0$ ). Weinberc

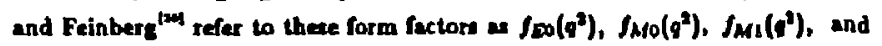
$f_{\Sigma 1}\left(g^{2}\right)$ reapectively (up to factore of $q^{2}$ etc.). The fow term is actually a

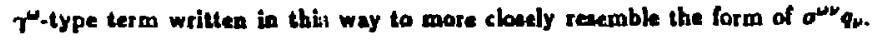
This followe from

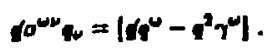

We see that the $P_{1}, f_{i}^{t}$ term $\left(f_{\infty} . f_{\nu 10}\right)$ will not contribute for real, free, masales

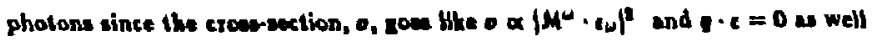

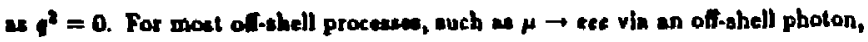
the term will vanieb is any case. With this in mind it is not surprining that the leadinz-order confictiente of $F_{i}\left(\varphi^{2}\right)$ and $F_{1}^{2}\left(\varphi^{2}\right)$ are found to vanich.

\subsection{Decay Rat:}

\section{SQED Reeull}

From Appendix $\mathbf{O}$ we find that

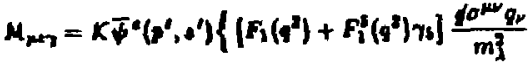

$$
\begin{aligned}
& \left.+\left[F_{2}\left(q^{2}\right)+F_{2}^{k}\left(q^{2}\right) \gamma_{s}\right] \sigma^{\mu \nu} q_{\nu}\right\} \psi^{\mu}(p, s)
\end{aligned}
$$

with

$$
K=-\frac{Q^{9 / 2} \sin \operatorname{cose} 0}{114 \sqrt{\pi}} \quad Q_{0}=P_{u}-P_{\omega}^{\prime}
$$

and that, when the muon decaye radiatively on-ahell to a photon and electron 
that the $F_{1}$ and $F_{f}^{*}$ terms are unimportant but that

$$
\begin{aligned}
& F_{z}\left(q^{3}=0\right) \doteq \frac{D m_{\psi}}{m_{\lambda}^{3}}\left[\Delta f_{z}\left(T_{R}\right)+\Delta J_{2}\left(T_{L}\right)\right] \\
& F_{2}^{*}\left(q^{2}=0\right) \doteq \frac{D m_{\mu}}{m_{2}^{g}}\left|\Delta f_{1}\left(T_{R}\right)-\Delta f_{h}\left(T_{2}\right)\right|
\end{aligned}
$$

where $m_{\lambda}$ is the photino mass, which is not necessurily small, and we wre condidering a putely SQED seault. We hove abbreviated the man ratios (Chapter 3 and Appendix Ol to:

$$
T_{L}=\frac{m_{t_{t_{1}}}^{2}}{m_{\Lambda}^{2}} \quad T_{R}=\frac{m_{i_{p_{1}}^{2}}^{2}}{m_{l}^{2}}
$$

From the eppendix

$$
\begin{aligned}
& \Delta f_{2}\left(T_{R}\right) \equiv f_{2}\left(\frac{m_{t_{1}}^{*}}{m !}\right)-f_{2}\left(\frac{m_{R_{1}}^{l}}{m !}\right) \\
& \Delta J_{2}\left(T_{L}\right) \equiv J_{2}\left(\frac{m_{L_{1}}^{2}}{m_{\lambda}^{2}}\right)-J_{2}\left(\frac{m_{L_{2}}^{l}}{m_{\lambda}^{l}}\right) \\
& f_{3}(x)=\frac{(1-x)\left(x^{2}-5 x-2\right)}{(1-x)^{4}}-f_{x}(1)=-\frac{1}{6} .
\end{aligned}
$$

This has been plotted in Fir. 4." Ne find that the decay width is

$$
\Gamma_{\text {mh }}=\Gamma_{0} \sin ^{2} \cos ^{2}\left(\frac{m_{M}}{m_{A}}\right)^{4}\left(\left|\Delta f_{2}\left(T_{L}\right)\right|^{2}+\left|\Delta f_{2}\left(T_{n}\right)\right|^{2}\right)
$$

where

$$
\begin{aligned}
\Gamma_{0} & \equiv \frac{a^{2}}{1024 x^{2}} \frac{m_{\mu} e^{2}}{h} \\
& \simeq 6.17 \times 10^{12} \sec ^{-1}
\end{aligned}
$$

\section{Small Mass-Splittint}

In the case of small alepton mass-aplittingt, when

$$
\begin{aligned}
& \Delta f_{2}\left(T_{L}\right)=\delta_{\ell_{L}} f_{g}^{\prime}\left(T_{L}\right) \quad \Delta h_{2}\left(T_{R}\right)=\delta_{\ell_{R}} f_{g}^{\prime}\left(T_{R}\right) \\
& \delta_{L_{L}}=\frac{m_{L_{L_{1}}}^{*}-m_{l_{L_{2}}}^{2}}{m_{l_{l_{1}}}} \quad f_{f}^{\prime}\left(T_{L}\right)=T_{L} \frac{\partial f_{L}\left(T_{L}\right)}{\partial T_{L}} \\
& \delta_{i_{R}} \equiv \frac{m_{i_{n_{1}}}^{2}-m_{i_{n_{1}}}^{2}}{m_{i_{n_{1}}^{2}}^{2}} \quad f_{f}^{\prime}\left(T_{R}\right)=T_{R} \frac{\partial J_{h}\left(T_{R}\right)}{\partial T_{R}} \\
& i_{L}<1 \quad \dot{i}_{n}<1
\end{aligned}
$$

we have

$$
r_{\text {per }} \doteq \Gamma_{0} \sin ^{2} \theta \cos ^{2} \bullet\left(\frac{m_{A}}{m_{\lambda}}\right)^{d}\left[\delta_{l_{L}}^{2}\left|f_{L}^{\prime}\left(T_{L}\right)\right|^{2}+\delta_{l_{R}}^{2}\left|f_{1}^{\prime}\left(T_{R}\right)\right|^{2}\right]
$$

Nole that

$$
\begin{aligned}
f_{2}^{\prime}(x)=\frac{x}{3(1-x)^{3}}\left\{(1-x)\left(x^{2}-8 x-17\right)-\theta(6 x+1) \ln x\right\} \\
f_{2}^{\prime}(0)=0 \quad f_{j}^{\prime}(x \rightarrow 0) \sim-\frac{1}{3} x(17+6 \ln x) \\
f_{2}^{\prime}(1)=\frac{1}{10} \\
f_{2}^{\prime}(\infty)=0 \quad f_{2}^{\prime}(x \rightarrow \infty) \sim \frac{1}{3 x}
\end{aligned}
$$

The peak of $f$ 'it lound al

$$
f_{3}^{\prime}(0.273)=0.12407
$$


contribution will be maximal when

$$
m_{\lambda} \propto 2 m_{i}
$$

Note that $f\left(T_{L}\right)=-2 J_{(z(2-z)]}\left(T_{L}, 0\right)$ which is given in Table $L .1$.

\section{Electroweak Calculation}

As discussed in the arut section, in a [ull superaymmetric aloctrow enk (SGWS) calculation, with in this model, the only caugino which would cortribute would be a pure $\tilde{B}^{0}$, thould it be 0 mass eigenalnte.

Thus the calculation would be identical with thet of the purely SQED can except that the photino $\lambda(\tilde{\gamma})$ would be uupplented by a Bino. Higetino contributions would enter into $M_{\mu-1}^{4}$ with a factor of $m_{j}^{4}$ down even from the $m_{m}^{2}$ coefficients which allowed us to lenoze the $F_{l}$ and $F_{i}$ torm. In uning a photino we have been, in effect, uring only a piee of the full $\bar{B}^{0}$ coatribution (i.e. W the $\tilde{\boldsymbol{\gamma}}-\tilde{z}^{0}$ were the correct mas eigenbals we would have been reglecting the 2 ino termu). The Bino and photino couplinge are simllas excopt thet we muat make the following eubetitutions:

$$
\begin{array}{lllll}
i e \sin \theta_{i} i e \cos \theta & \rightarrow & -\frac{i}{2} \rho^{\prime} \sin \theta_{i}-\frac{i}{2} \rho^{\prime} \cos \theta & \text { for } & t_{L} \\
\text { ie } \sin \theta_{i} i c \cos \theta & \rightarrow & i g^{\prime} \sin \theta_{i} i g^{\prime} \cos \theta & \text { for } & t_{R}
\end{array}
$$

where $g^{\prime}=\frac{\varepsilon}{\cos }$ is the $U(1)_{Y}$ hypercharge coupliag constant $\left(Y_{C_{L}}=-1\right.$; $\left.Y_{e_{n}}=2\right)$. Usine this (4.0) becomes

$$
r_{\mu+\gamma}=r_{0} \frac{\sin ^{2} \theta \cos ^{2} \theta}{\cos ^{2} \theta_{w}}\left(\frac{m_{\mu}}{m_{D^{\prime}}}\right)^{\dagger}\left(\frac{\left|\Delta f_{2}\left(T_{L}\right)\right|^{2}}{16}+\left|\Delta f_{2}\left(T_{R}\right)\right|^{2}\right) .
$$

This is exactly correct if $\vec{B}^{0}$ is indeed an eigenstate. I the photino and Zino are closer to the true eigenbuses then we would use the SQED result and add in a similar $\tilde{z}^{0}$ contribution obtainable by the following subntitutione from the SQED reault:

$$
\begin{aligned}
& \text { iesin } \theta_{i} i r \cos \theta \quad \rightarrow \quad-i e \cot 2 \theta_{w} \sin \theta_{i}-i e \cot 2 \theta_{w} \cos \theta \text { for } \bar{C}_{L}
\end{aligned}
$$

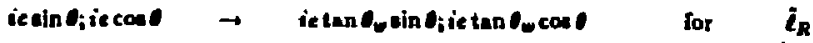

which follows from the general termion-fermion- $Z^{0}$ vertex

$$
\frac{\theta}{\cos \theta_{-}}\left[T_{2}-Q \sin ^{2} \theta\right]
$$

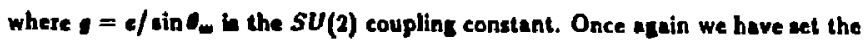
left and sight mixing ancles equal (to 'o').

Pursuing thin line of resconing further the Integral function terme may atso be readily extended to laclude both photinos and zinos:

$$
\begin{aligned}
& \frac{\Delta f_{2}\left(T_{2}\right)}{m_{\lambda}^{l}} \rightarrow \frac{\Delta f_{2}\left(T_{l}^{\lambda}\right)}{m_{\lambda}^{2}}+\cot ^{2} 20_{-} \frac{\Delta f_{2}\left(T_{l}^{l^{+}}\right)}{m_{2}^{2}} \\
& \frac{\Delta f_{2}\left(T_{k}\right)}{m_{l}^{2}} \rightarrow \frac{\Delta f_{2}\left(T_{l}^{\lambda}\right)}{m_{l}^{2}}+\tan ^{2} \theta_{\frac{1}{2}} \frac{\Delta f_{2}\left(T_{h}^{k}\right)}{m_{k}^{2}}
\end{aligned}
$$

where

$$
\begin{aligned}
& T_{\Sigma}^{\lambda}=\frac{m_{i_{L_{1}}}^{2}}{m_{\lambda}^{2}} \quad T_{R}^{A}=\frac{m_{i_{n_{1}}}^{2}}{m_{\lambda}^{2}}
\end{aligned}
$$

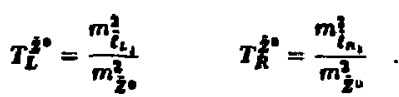


The width then becomes

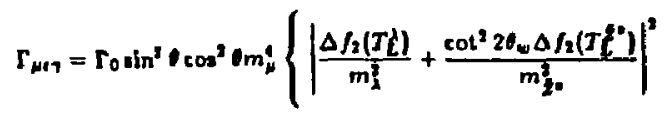

$$
\begin{aligned}
& \left.\left|\frac{\Delta f_{2}\left(T_{h}^{\lambda}\right)}{m_{\lambda}^{2}}+\frac{\tan ^{2} \theta_{w} \Delta f_{2}\left(T_{h}^{(1)}\right)}{m_{2}^{2}}\right|^{2}\right\}
\end{aligned}
$$

It is clear how we would extend this lo the Inatunce of ceneral neutralino mixint in the fashion used in chapter three and its asoctaled appendices.

\subsection{COMPARISON WITH EXPERIMENT}

The radiative decay of the muon hes been intensively wearched for. Thus far no positlve evidence has curfaced but Improved limits are beins reposted on a regular basis. We will use the limit ${ }^{\text {Ial }}$

$$
\frac{\Gamma(\mu \rightarrow e \gamma)}{\Gamma(\mu \rightarrow e \nu \nu)}<4.9 \times 10^{-11}
$$

and

$$
r_{\text {TOT }} \approx r_{x \rightarrow \text { ant }}=455137 n^{-1}
$$

therefore

$$
r_{p+e y}^{+2 p p} \leq 2.2 \times 10^{-3} s^{-1}
$$

and wo

$$
\Gamma_{m \rightarrow .9}^{\ln } / \Gamma_{0} \leq 3.6 x^{-10}
$$

It must be commented that (1.24) represents a remukably accurste limit and is the culruination of much dedicated work by many people.
From (4.9) and (4.18) we may immedintely obtain

$$
\sin ^{2} \cdot \cos ^{2} \bullet\left(\frac{m_{\mu}}{m_{\lambda}}\right)\left(\left|\Delta / J_{l}\left(T_{L}\right)\right|^{2}+\left|\Delta / \lambda\left(T_{R}\right)\right|^{2}\right) \leq 3.6 \times 10^{-10}
$$

for SQED and

$$
\frac{\sin ^{2} \theta \cos ^{2} \theta}{\cos ^{4} \theta_{\omega}}\left(\frac{m_{L}}{m_{b}}\right)^{4}\left(\frac{\left|\Delta / 2\left(T_{L}\right)\right|^{2}}{16}+\left|\Delta / 2\left(T_{R}\right)\right|^{2}\right) \leq 3.6 \times 10^{-10}
$$

for SGWS if the Bino were mase eigenatale. Nole that if

$$
m_{i_{1}} \simeq m_{i_{n_{1}}} \quad m_{i_{i_{2}}}=m_{i_{n_{1}}} \quad v=\frac{\pi}{4}
$$

that (SQED)

$$
\frac{\left|\Delta f_{2}\left(T_{L, n}\right)\right|}{m_{\lambda}^{3}} \leq 2.4 \times 10^{-7} \mathrm{GeV}^{-3}
$$

\section{Small Mass-Splitting Limik}

If the difference in the mass of $m_{i_{1}}$ and $m_{I_{L_{2}}}$ is amall (and the ame for the "right" oleptons), i.e.

$$
{ }^{6} i_{L}<1 \quad \sigma_{i_{A}}<1
$$

in (4.11) then we have

$$
\Gamma_{\mu \mathrm{r} 7}=\Gamma_{0} \sin ^{2} \cdot \cos ^{2} *\left(\frac{m_{H}}{m_{\lambda}}\right)^{4}\left[\delta_{\frac{1}{\ell_{L}}}\left|f_{i}\left(T_{L}\right)\right|^{2}+\delta_{\ell_{R}}\left|f_{L}^{\prime}\left(T_{R}\right)\right|^{2}\right] .
$$

Since $f_{2}^{\prime}(0)=f_{2}^{\prime}(\infty)=0$ we know that

$$
\begin{aligned}
& \Gamma_{\mu \rightarrow+7}=0 \text { (i) } m_{\lambda} \rightarrow \infty \\
& \text { (ii) } m_{i} \rightarrow \infty \\
& \text { (iii) } m_{i,} \rightarrow m_{i,}
\end{aligned}
$$


From (4.16) $f_{2}^{\prime} \leqslant$. Should it be that

$$
\delta_{\bar{Z}_{L}} \simeq \delta_{\boldsymbol{i}_{R}} \approx \delta_{z}<1
$$

then, for SQED,

$$
\Gamma_{\mu \text {, }} S \frac{\sigma^{2}}{32} \Gamma_{0} \sin ^{2}+\cos ^{2} v\left(\frac{m_{\mu}}{m_{2}}\right)
$$

and since $\sin ^{2} \theta \cos ^{2} \theta \leq 1$

$$
\Gamma_{\mu 07} \leq \frac{q}{128} \Gamma_{0}\left(\frac{m_{\mu}}{m_{A}}\right)^{\prime}
$$

When we compare with experlment using (1.27) we obtain a limit on how large the relative splitting may be.

$$
\delta_{i} \leqslant 1.0 \times 10^{-6} \mathrm{~m}_{\lambda}^{2}
$$

where $m_{\lambda}$ is tiven in GeV. This should be compared with (4.30). Thus for $m_{\lambda} \sim 100 \mathrm{GeV}$ the slepton splitting would be las than $2 \%$ whike for $m_{\lambda} \sim 1 \mathrm{GeV}$ the slepton eplitting would be Jess than $2 \times 10^{-6 \%}$. These would occur near the meximal cross-section, when $m_{\lambda} \approx 2 m_{j}$.

The condition that $m_{\lambda} \approx 2 m_{i}$ is e rether unnatural one. A popular acenario amone designers of apecific models is that the neutrulino atate which is principally photino-like tends to be on the light side. Indeed some suthors tend to asume that $m_{\lambda}<m_{i}$ elmost axiomatically. Although we will not take this view it is, however, interestine to examine the results in this limit. if

$$
m_{\lambda}<m_{i_{L . n}}
$$

then

$$
T_{L, R} \rightarrow \infty
$$

nnd

$$
f_{2}^{\prime}\left(T_{L, \Omega}\right) \rightarrow \frac{1}{3 T_{L, \Omega}} .
$$

In this case (1.13) become

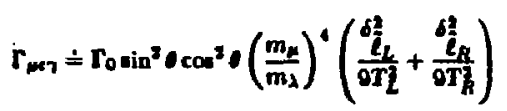

and so if, in addition, $\delta_{C_{L}} \simeq \delta_{i_{R}}$ then (SQED)

$$
\Gamma_{m \alpha \tau} \approx \frac{2}{\theta} \Gamma_{0 \sin ^{2}}+\cos ^{2} \cdot\left(\frac{m_{\mu}}{m_{\lambda}}\right)^{4} \delta \frac{2}{t_{L}}
$$

and if $\theta=\pi / 4$ (maximal mixing)

$$
\frac{\delta_{i}}{m_{i}^{3}} \leqslant 7.3 \times 10^{-7} \mathrm{GeV}^{-2}
$$

If the Bino is an eigenstate and $m_{j 0} \leqslant m_{i}$ this would become $\left(\cos ^{2} \theta_{w}=0.77\right)$

$$
\frac{6}{m_{t}^{2}} \leqslant 7.6 \times 10^{-7} \mathrm{GeV}^{-9} \text {. }
$$

In their classic paper ${ }^{\text {tza }}$ Ellis and Nanopoulos made the frat rough estimates for such processes. Working exclusively in the amall mass-splitting regime (us discussed in chapter three) they found

$$
\delta_{t} / m \frac{x}{l}<O\left(10^{-7}\right) G a V^{-2}
$$

assuming maximal mixing and that $\tilde{B}^{\circ}$ is an eigentate. At the time which that paper was written the best experimental limit was $\Gamma_{\mu \rightarrow c 7}^{0 \times p} / \Gamma_{T o r}<1.9 \times 10^{-10}$. 
Uaing this value (4.37) would aller to

$$
\frac{\delta_{i}}{m_{i}^{2}} \leqslant 3 \times 10^{-6} \mathrm{GeV}^{-2}
$$

which is a factor of thirty of of their naive ealimate. Thus a careful computation reveals that we are only able to place comewhat weaker limits than one might have initjally hoped.

\section{Lerze Mass-Splittin L Limit}

In this limit $m_{i_{L}}>m_{i_{L}}$ and $m_{i_{n_{2}}}>m_{l_{n_{i}}}$. Therelore

$$
\Delta f_{2}\left(T_{L, N}\right) \rightarrow f_{2}\left(T_{L, N}\right)
$$

Where $f_{2}(x)$ was given in $(4.8)$. Nole that

$$
f(x \rightarrow \infty) \rightarrow-\frac{1}{3 x}
$$

If we further consider the case where $m_{i}>m_{\dot{x}}$ then (for $\bar{x}^{0}=\bar{b}^{0}$ ) fram (4.18)

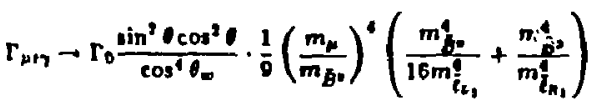

$$
\begin{aligned}
& =\frac{1}{9} r_{0} \frac{\sin ^{2} 1 \cos ^{2} \theta}{\cos ^{2} \theta_{0}} m_{m}^{4}\left[\frac{1}{16 m_{L_{1}}^{2}}+\frac{1}{m_{l_{n_{3}}}^{4}}\right]
\end{aligned}
$$

For $\theta=\theta_{L}=\theta_{R}=x / 4$ and using $\cos ^{2} \theta_{-}=0.77$ and $(1.27)$ we find

$$
\left(\frac{1}{16 m t_{L_{1}}}+\frac{1}{m_{i_{1}}}\right) \leq \frac{7.7 \times 10^{-18}}{m_{i}^{6}}
$$

Further specializing to

$$
m_{i_{L_{1}}}=m_{i_{n_{1}}} \equiv m_{i}
$$

would result in

$$
m_{i} 21.1 \mathrm{TeV}
$$

ince $m_{\nu}=0.105650 \mathrm{GeV}$.

This result is not "vioble" aince it was oblained by considering the limit $m_{i_{2}}, m_{i_{n}}>m_{i} \approx 1 \mathrm{TeV}$ and we would like the ouperaymmetry-breaking acale to be $5 O(1 \mathrm{TeV})$ if it is to alleviate the gauge hiefarchy problem in a 'natusal' way. If the rass eizenstates are photinos and Zinos we ahould anticipate a similar reault in this limit. If $m_{g_{\bullet}} \sim m_{z}$ then $m_{i}>m_{\lambda, z^{*}}$ and since $T_{l}^{\lambda}=T_{R}^{\lambda}$ and $T_{\ell}^{\prime \prime}=T_{F}^{*}$ we would have from (4.18)

$$
\Gamma_{\mu \mathrm{r} \gamma} \rightarrow \Gamma_{0} \sin ^{2} \cos ^{2} \theta+\frac{1}{9}\left(\frac{m_{\mu}}{m_{i_{L}}}\right)^{4}\left\{\left(1+\cot ^{2} 2 \theta_{w}\right)^{2}+\left(1+\tan ^{2} \theta_{w}\right)^{2}\right\}
$$

The term in the curly brackets ts about 3.7. If only the photino part were included It would have been $\mathbf{2 . 0}$ while the corresponding term for the Bino was about $\mathbf{1 . 8}$ In the photino-Zino case the limit in (4.41) would become

$$
m_{i} \geq 1.4 \mathrm{TeV}
$$

Such a constraint would, of course, be weakened if the angular mixing were not maximal.

\section{Implication of ASP ResulL}

The ASP sincle pholon wearch at SLAC has placed"ll limils on $m_{\dot{c}}$ of about $60 \mathrm{GeV}$. When combined with the results of MAC and other collaboration they 
find (100) (under a eat of posameter assumptions) that

$$
m_{\text {Z }} \geq 84 \text { GeV. }
$$

The limit comes from considerations of the proces

$$
e^{+} e^{-} \rightarrow \boldsymbol{\text { พิก }}
$$

whose leading contribution arisen from the diagram in Fig. 4.3. Under the assumptions that $m_{\bar{c}_{L}}=m_{\bar{e}_{n}}$, the photino is a mase wigenstate and is the only light neutral gaugino, and that $m_{\tilde{i}}<m_{i_{L, R}}$, they quote

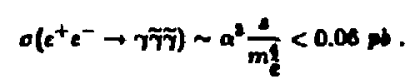

The specific search is for $e^{+} e^{-} \rightarrow$ t tenergy where the standard model calculation of $e^{+} e^{-} \rightarrow \gamma \nu \bar{D}$, for three light neutrino generations, hes been accounted for. Thus the search for new light epecier of neutrinos (2s well as Hiksuinos, aneutrino and 10 forth) compliments the phoilno cearch, wace the existence of auch otstes would only improve the limit in (4.43).

The above analysic asumes that there is no intercenerational mixing. Por-

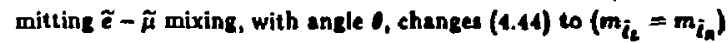

$$
\sigma\left(c^{+} e^{-} \rightarrow \gamma \tilde{\gamma} \tilde{\gamma}\right) \sim a^{3} \cdot\left(\frac{\cos ^{2} \theta}{m_{l_{1}}^{2}}-\frac{\sin ^{2} t}{m_{f_{1}}^{2}}\right)^{2}<0.00 \omega
$$

so instead of

$$
\frac{1}{m_{E}^{2}}<\frac{1}{(84 G a V)^{2}}
$$

which follows from (4.43) we have

$$
\frac{\cos ^{2} \theta}{m_{i_{1}}^{2}}-\frac{\sin ^{2} \theta}{m_{i_{2}}^{2}}<\frac{1}{(84 G e V)^{2}}
$$

Since $\sigma(\mu \rightarrow \epsilon \gamma)$ goes as $\sin ^{2} \theta \cos ^{2} \theta$, whereas $\sigma(e \varepsilon \rightarrow \gamma \tilde{\gamma})$ has $\theta$ dependence as in (1.16), we see that, when thee proceses are compared, that the mixing angle $\left(0=\theta_{L}=\theta_{R}\right)$ entere non-trivially.

First let us considar the case of large mast-spliuing, where $m_{i_{1}}>m_{i_{1}}$.

Then (4.46) raduces to

$$
\frac{\cos ^{2} \theta}{m_{i_{1}}^{2}}<\frac{1}{(4 G c V)^{2}}
$$

i.e.

$$
m_{i,}>24 G \mathrm{Cos} \theta
$$

which is weaker that $(4,43)$. For $1=\pi / 4$ (maximal mixind) this limit becomes

$$
m_{i_{1}}>\mathrm{BOGeV} \text {. }
$$

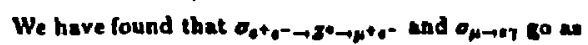

$$
\sin ^{2}\left(\cos ^{2} \varphi\left(\Delta F_{1}\left(T^{\theta}\right)+\Delta F_{2}\left(T^{+}\right)\right)\right.
$$

where $T^{0}=\frac{m_{l}^{2}}{m_{\lambda}^{2}}$ and $T^{+}=\frac{m_{\vec{\nu}_{1}}^{2}}{m_{i}^{2}} . \Delta F_{1,2}$ are come functions which decreace with large, incressing arguments. For $\mu \rightarrow e^{\prime}$ the $F_{2}$ term is absent. Thus as the limit (rom (4.47) becomes stronger (increasing $\cos \theta$ ) then $\left.\max \mid \Delta F_{1}\right]$ decreases. However $\sin ^{2} \theta \cos ^{2} \theta$ pents at $\theta=\pi / 4$. Thus $a t$ increase from $\theta<\pi / 4$ to $0=\pi / 4, \max \left|\Delta F_{1}\right|$ decreases while $\operatorname{din}^{2} \theta \cos ^{2} \theta$ increases. As $O$ increases beyond $0=\pi / 4 \max \left|\Delta f_{1}\right|$ increaset while $\sin ^{2} \theta \cos ^{2} \|$ decreases. Thus there is alwaye competition between these two effects (as far as obtaining experimental limits from $e^{+} e^{-} \rightarrow \mu^{+} c^{-}$and $\mu \rightarrow$ er are concerned). Note that at $\cos \theta=0,1$ that both processes vanish. 
Let us now consider the small mass-splitting case:

$$
m_{i_{1}}^{2}-m_{i_{1}}^{2} \equiv m_{i_{1}}^{2} \cdot \delta_{i}<m_{i_{13}}^{2} .
$$

Then

$$
\frac{1}{m_{l_{1}}^{2}}=\frac{1-\delta_{i}}{m_{l_{1}}^{2}}
$$

and therelore

$$
\frac{\cos ^{2} \theta}{m_{l_{1}}^{2}}-\frac{\sin ^{2} \theta}{m_{l_{1}}^{2}}-\frac{\cos ^{2} \theta-\sin ^{2} \theta}{m_{l_{1}}^{2}}+\frac{\sin ^{2} \theta \delta_{i}}{m_{l_{1}}^{2}}
$$

If we are near "maximal mixing" then $\leq \pi / 4$ and

$$
\frac{\cos ^{2} t}{m_{t_{1}}^{2}}-\frac{\sin ^{2} t}{m_{t_{3}}^{2}}=+\frac{b_{i}}{2 m_{i_{1}}^{2}}
$$

and (4.46) becomes

$$
\frac{\delta_{i}}{2 m_{i_{1}}^{2}} \leq \frac{1}{(84 G \mathrm{eV})^{2}}
$$

i.e.

$$
\frac{\delta}{m_{\bar{f}_{1}}^{\delta_{i}}} 53 \times 10^{-4} \mathrm{GeV}^{-2}
$$

which is much weaker than the $\mu \rightarrow$ er limit

$$
\frac{\delta_{i}}{m_{t_{i}}^{i}} \leqslant 7 \times 10^{-7} \mathrm{GeV}^{-2}
$$

which we lound in (1.36).
The weakness of the limit lies in the supposition that $=\pi / 1$. This is due to the particular form of (4.49). If we took $=\pi / 3$ then the limit in (1.36) would chenge to

$$
\frac{\delta_{\bar{l}}}{m_{\bar{l}_{1}}^{2}} \leqslant 10^{-4} \mathrm{GeV}-2
$$

from $\mu \rightarrow$ ey while $m_{f_{1}}>42 \mathrm{GeV}$ from $e^{+} e^{-} \rightarrow r \overline{7}$. When 6 not near $\pi / 4$ (4.46) and (4.49) imply that

$$
m_{i_{1}}>84 \mathrm{GeV} \cos 20
$$

us opposed to (1.47). Nate that timils that ASP places on $m_{w}$ from the enalogous proceas $\varepsilon^{+} e^{-} \rightarrow$ qü are both weaker and subject to a creater number of modeldependent assumptions. 


\section{FIGURE CAPTIONS}

1. The vertex $\gamma \rightarrow$ Ae.

2. (a) The function $f_{2}(x)$ ขa. $x$

(b) The function $f_{j}^{\prime}(x)$ va. $x$.

3. Prineiple contribution lo the procese

$$
e^{+} e^{-} \rightarrow \text { 7กับ }
$$

used by ASP to place limits on the seleciron man.

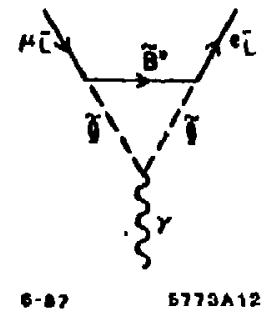

Fig. 4.1 


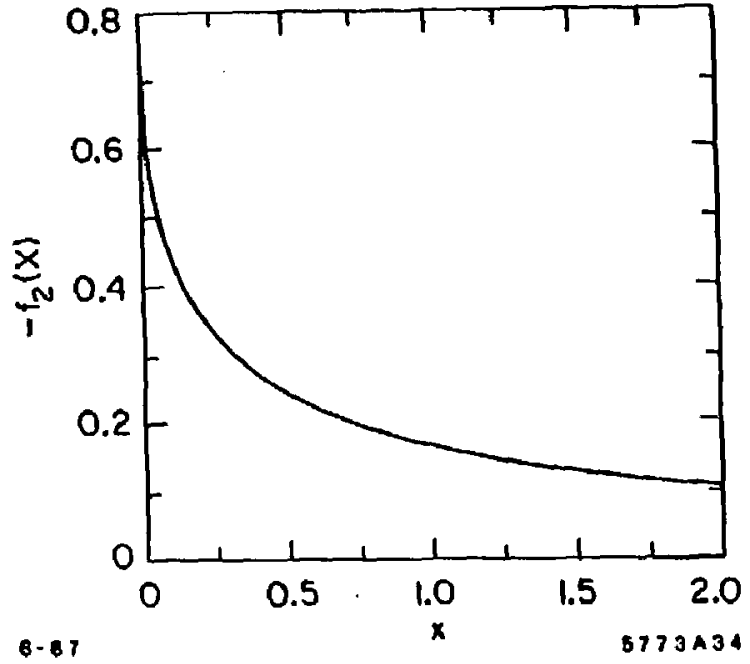

Fig. $4.2 \mathrm{a}$

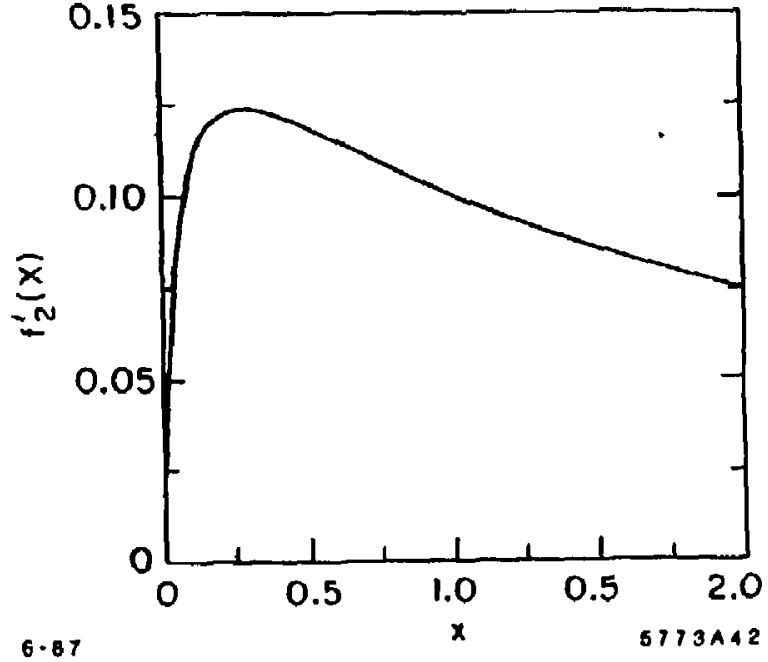

Fig. $4.2 b$ 


\section{Summary}

We have reen that intergeneretional mixing of sleptons may octur at experimentally accepteble rates. When compared wilh experiment we may betin to eliminale certain rezloas from an otherwien unconstrained parnmeler space. It it not anticipated that kplon family aumber violation, arieing from this mechaniem, will be seen at the SLC due to insufifichnt production rater.

The low-enerey procmese of chapters two and four provide more substantial constraints. I the maen-eplitting between the two sectors (left-right or apecies one-species two) \& amall then reasanably strons limits on the eplitting may be obtained, depending upan the specific involved. If a large masesplitting is esumed then some quite reapectable limits of oupersymmatric mases become possible, but this is exaln highly dependent upon opecifics.

In conclusion we fol that the eeusch for both family number non-conervation and evidence for the exintense of supereymmatry is of ereat importance. Then interplay between these two ares can provide endless entertainment for both experimentalists and theorints alike.

Fig. 4.3 


\section{APPENDIX A}

\section{Two-Coroponent Weyl Notation}

The two-component Weyl cpinor formalism is a more fundamental representation of spinorial states and algebra than that of Dirac. In mont theories the Dirac nolation is a convenient ahorthand for oummarizing the aclions of the difierent chiral states and this explains its iramense utility and popularity. In eupersymmetric theories the states of difierent chirality are dealt with independently and two-component notation is the natural longuace to use. Greater familiarity with the manipulation of Dirac objects will prompt us to use them in the more involved computations but the irrecusable consequence will be the proliferation of conjugation and helicity projection operators.

There sre many excellent seferences 1,12,1" whose sppendices aummarize definitions (which vary from one author to the nexi) and properties of the Weyl algebra. We will use notation similar to that of reference 12 . The most approprinie representation of the gamma matricss to use when we work in four-component notation is not the Dirac representation but the chiral representation. Note that the chiral tepresentation used here differs from that of Itzykson and Zuber.

\section{A.1 SPINORS}

We write the electron as:

$$
\begin{aligned}
& \psi=\left(\begin{array}{l}
\varphi_{0} \\
\bar{x}^{d}
\end{array}\right) \equiv\left(\begin{array}{ll}
\psi_{-\bullet} \\
\bar{\psi}_{+}^{d}
\end{array}\right) \quad \begin{array}{l}
\text { L.H. } e^{-} \\
\text {R.H. } e^{-}
\end{array} \\
& \bar{\psi}=\left(\begin{array}{ll}
x^{a} & \bar{\phi}_{d}
\end{array}\right) \equiv\left(\begin{array}{ll}
\psi_{+}^{a} & \bar{\psi}_{-d}
\end{array}\right) \\
& \text { L.H. } \mathrm{e}^{+} \text {R.H. } \mathrm{e}^{+}
\end{aligned}
$$

indices denote right-handed $(0,1)$ representations of the Lorents Group. Unbarred spinors $(\psi)$ and indotted indices denote left-finded $(\xi, 0)$ representations.

\section{A.2 Sigma and Gamma Matajces}

The metric ured in $\sigma^{m}=\left(\begin{array}{cccc}1 & 0 & 0 & 0 \\ 0 & -1 & 0 & 0 \\ 0 & 0 & -1 & 0 \\ 0 & 0 & 0 & -1\end{array}\right)$

The gamma matrices, $7^{\mu}$, heve the atructure

$$
\gamma^{\mu}=\left(\begin{array}{cc}
0 & \left(\sigma^{\mu}\right)_{-\alpha} \\
\left(\partial^{\mu}\right)^{\alpha \sigma} & 0
\end{array}\right) \quad \mu=0,1,2,3 \quad \gamma^{\prime}=\left(\begin{array}{cc}
-1 & 0 \\
0 & 1
\end{array}\right)
$$

where

$$
\begin{array}{ll}
\sigma^{0}=\left(\begin{array}{ll}
1 & 0 \\
0 & 1
\end{array}\right) & \sigma^{\prime}=\left(\begin{array}{ll}
0 & 1 \\
1 & 0
\end{array}\right) \\
\sigma^{2}=\left(\begin{array}{cc}
0 & -i \\
i & 0
\end{array}\right) & \sigma^{3}=\left(\begin{array}{cc}
1 & 0 \\
0 & -1
\end{array}\right)
\end{array}
$$

Definition:

$$
\sigma^{\mu \nu}=\frac{i}{2}\left|\gamma^{\mu}, \gamma^{\nu}\right| \text { (1-component) }
$$

D) Note that Wess and Bagers' une

$$
\eta^{\mu \nu}=\left(\begin{array}{cccc}
-1 & 0 & 0 & 0 \\
0 & 1 & 0 & 0 \\
0 & 0 & 1 & 0 \\
0 & 0 & 0 & 1
\end{array}\right) \text { and } \sigma^{0} \approx-\left(\begin{array}{cc}
1 & 0 \\
0 & :
\end{array}\right)
$$

where the position of the indices in conventional. Barred spinors $(\psi)$ and dotted 


$$
\begin{aligned}
& \sigma^{\mu \nu}=\frac{i}{2}\left(\partial^{\mu} \sigma^{\nu}-\partial^{\nu} \sigma^{\mu}\right] \quad \text { (2-component) } \\
& \sigma^{\mu \nu}=\frac{i}{2}\left|\rho^{\mu} \theta^{\nu}-\sigma^{\nu} \partial^{\mu}\right| \text { (2-component). }
\end{aligned}
$$

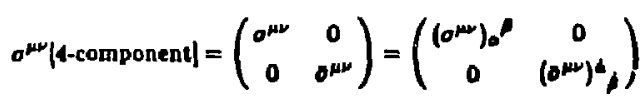

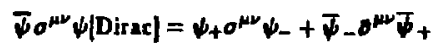

$$
\begin{aligned}
& A=a \cdot b-i e_{\mu} b_{k} a v
\end{aligned}
$$

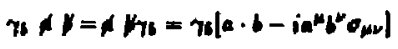

$$
\begin{aligned}
& (o \cdot p)(a \cdot q)=p \cdot q-i o^{\mu \nu p \mu q u}
\end{aligned}
$$

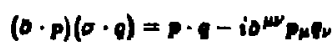

A epecinl cese is

$$
(a \cdot p)^{2}=\left(\sigma^{\mu}\right)_{\alpha} p^{p}(a)^{j} p_{p_{\nu}}=p^{2} \delta_{\alpha}^{\alpha}
$$

\section{A.3 INDEX GMANASTICS}

Raise and lower indices with a as follows

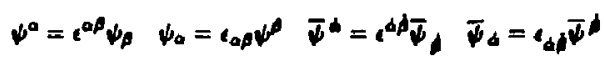

$$
\begin{aligned}
& A^{\alpha A}=\left(\begin{array}{cc}
0 & 1 \\
-1 & 0
\end{array}\right) \quad C_{\alpha \beta}=\left(\begin{array}{cc}
0 & -1 \\
1 & 0
\end{array}\right) \quad \text { (in matrix form) }
\end{aligned}
$$

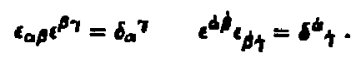

In axtuality $\Delta^{H}=\sigma^{H}$. $\left(\partial^{H}\right)^{d a}$ is just the saised index version of $\left(a^{\mu}\right)_{a d}$ and no more. Once we have chosen the convention that $\left(\sigma^{\mu}\right)$ has index atrurture $\left(\sigma^{\mu}\right)_{\text {ad }}$ then (om) always refers to the operator of complementery index ordering.

$$
\begin{aligned}
& \left(0^{\mu}\right)^{d \pm}=e^{\Delta+\epsilon^{n+1}\left(0^{\mu}\right)}
\end{aligned}
$$

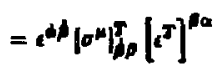

$$
\begin{aligned}
& \left.=-\left(c \mid \alpha^{\mu}\right]^{T} t\right)^{d a} \\
& =\left(\sigma^{2}\left|\sigma^{\mu}\right|^{T} \sigma^{2}\right)^{d \sigma}
\end{aligned}
$$

aince $t^{\circ \rho}=i\left(0^{2}\right)^{\circ \rho}$. However

$$
\left(0^{2},\left(0^{i}\right)^{5}\right)=-25^{i 2} \quad\left[0^{2},\left(0^{0}\right)^{5}\right)=0 \quad i=1,2,3
$$

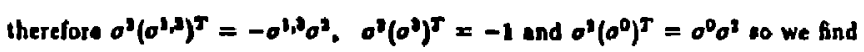
that

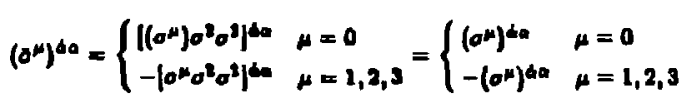

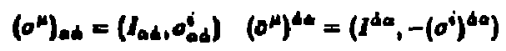

where $I=\left(\begin{array}{ll}1 & 0 \\ 0 & 1\end{array}\right)=\sigma^{0}$ is the unit matrix. Since $\left(\sigma^{\mu}\right)_{\text {ed }}=\left(1, \sigma^{i}\right)_{\text {ad and }}$

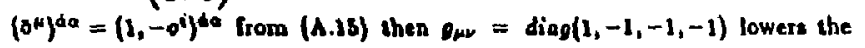
Lorentz index to tive

$D_{\mu \nu}\left(0^{\nu}\right)_{\alpha d} \equiv\left(\sigma_{\mu}\right)_{a d}=\left(1,-0^{i}\right)_{\alpha d} ; \operatorname{s\mu \nu }_{\mu \nu}\left(0^{\nu}\right)^{d a} \equiv\left(0_{\mu}\right)^{d a}=\left(1,0^{i}\right)^{d d}$.

Note that when we raise one index and lower another

$$
\bar{x}_{d} \bar{\psi}^{d}=-\bar{x}^{d} \bar{\psi}_{\boldsymbol{\alpha}}
$$


Notalion:

$$
x^{0} \phi_{a} \pm x \phi
$$

but

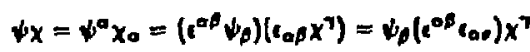

$$
\begin{aligned}
& =\psi_{\rho}\left(-e^{\beta a_{a_{1}}}\right) X^{\top}=-\psi_{\rho} \delta^{\rho}{ }_{T} X^{\top} \\
& =-\boldsymbol{v}_{\boldsymbol{\rho}} x^{*}
\end{aligned}
$$

$\psi^{\circ} x_{0}=-\psi_{0} x^{0} \approx x^{0} \psi_{0}$ since $x, \psi$ are fermions $=x \psi$. Thus

$$
\psi x=x
$$

A.4 Conjugation

$$
\begin{aligned}
& \left(\psi_{a}\right)^{\dagger}=\left(\psi^{\dagger}\right)_{\alpha}=\bar{\psi}_{\alpha} \quad(2 \text { component spinors }) \\
& C=\left(\begin{array}{cc}
\cos _{0} & 0 \\
0 & e^{\delta \rho}
\end{array}\right) \quad \text { (charge conjugation operator) } \\
& \psi^{C}=C \bar{\psi}^{7} \text { in ceneral } \\
& x_{0}=\cos (X)^{1 \rho}
\end{aligned}
$$

also note

$$
(x \boldsymbol{w})^{t}=\bar{\psi} \bar{x}=\bar{x} \bar{v}
$$

\section{A.6 USEFUL RELATIONSHIPS.}

A plethora of useful selationships may be found in Wess and Bazcer "Supersymmetry and Supereravity ${ }^{m 1}$. Some of these and others of intereat ase (here $m, n=0,1,2,3):$

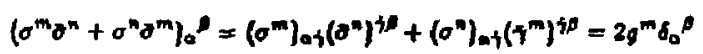

$$
\left(\theta^{m} \sigma^{n}+\theta^{m} \sigma^{m}\right)^{\phi} d=2 \theta^{m n} \sigma^{b} d
$$

$$
\operatorname{tr} \sigma^{m} a^{n}=\left(0^{m}\right)_{o j}\left(\theta^{n}\right)^{\beta_{0}}=2 g^{m n}
$$

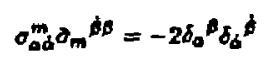

$$
\left(0^{m}\right)^{d_{0}}\left(a_{m}\right)_{\rho \phi}=-2 \delta^{\alpha} \beta^{\sigma^{\circ}}
$$

$$
\sigma_{a d}^{m} \delta_{m}^{b *}=-2
$$

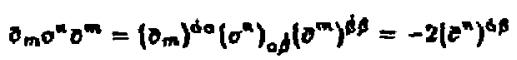

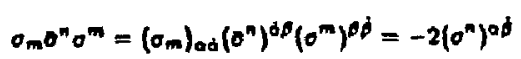

$$
a^{m} \partial^{n} a_{\infty}=-2\left(a^{n}\right)_{a d}
$$

$$
\partial^{m} \sigma^{n} \partial_{m}=-2\left(a^{n}\right)^{\beta B}
$$

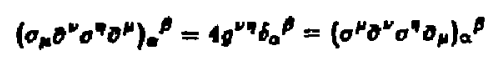




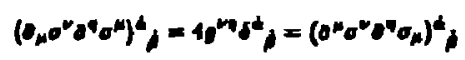

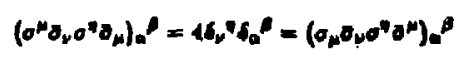

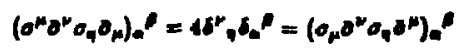

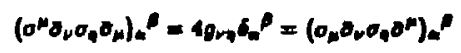

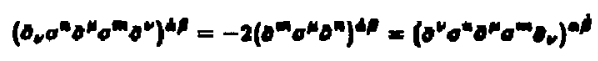

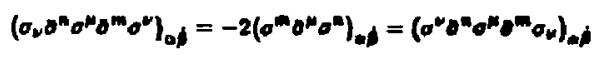

(A.3)

[A.37]

(A.30)

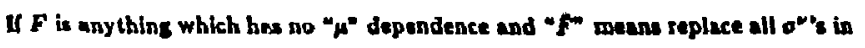

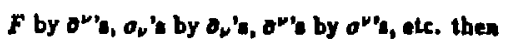

$$
\begin{aligned}
& { }^{M} F \sigma_{\mu}=O_{M} F O_{\mu}
\end{aligned}
$$

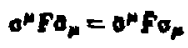

$$
\begin{aligned}
& \sigma_{\mu} F \sigma^{\mu}=\partial_{\mu} F \sigma^{\mu} \\
& \sigma_{\mu} F O^{\mu}=\partial_{\mu} F O^{\mu} \\
& F_{1} 0^{\mu} F_{2}=F_{3} 0^{\mu} F_{1} \Rightarrow F_{1} 0^{\mu} F_{2}=F_{3} 0^{\mu} F_{1} \\
& F_{1} \sigma^{M} F_{2}=F_{2} \phi^{M} F_{2} \Rightarrow F_{1} \otimes^{M} F_{2}=F_{3} \sigma^{*} F_{1} .
\end{aligned}
$$

In addition there are beatlition in reference 1 auch an

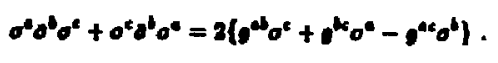

(A.41)

Fierz Rearrangement Formula:

$$
(\omega)^{\prime} \bar{x}_{i}=-1\left(6 \sigma^{m} \bar{x}\right)\left(4 \sigma_{m}\right)_{j}
$$

(A.42)

Prool:

$$
\begin{aligned}
& -1\left(\omega^{m} x\right)\left(\omega_{-m}\right)_{j}=-\frac{1}{2}\left(\phi^{\alpha}\left(\sigma^{m}\right)_{a c} x^{\alpha}\right)\left(\psi^{\rho}\left(\sigma^{m}\right)_{\rho j}\right) \\
& =-\mid\left(\psi^{\mu} \phi^{\mu} \bar{x}^{\alpha}\left(\sigma^{m}\right)_{\alpha d}\left(\sigma_{m}\right)_{p \beta}\right)
\end{aligned}
$$

and from (A.20) and (A.14)

$$
-\mid\left(\omega^{\infty} \bar{x}\right)\left(\psi \sigma_{m}\right)_{j}=-1\left(-2 \psi^{\omega} \phi \rho x_{p}\right\}=(\psi \phi) x_{p}
$$

as required.

Using (A.39) and (A.40) we can generate many more identities like (A.28)- (A.38).

73

$\boldsymbol{7}$ 


\section{APPENDIX B}

Two-Component QED

We use the two-corponent Weyl notation, a presented in Appendix A, to express the QED Lagrangian and Fejnman rules.

\section{B.1 Dirac equation}

$\left(\gamma^{\mu} \vec{p}_{\nu}-m\right) \cdot\left(p_{v} v\right)=0=\bar{v}\left(p^{\prime} v^{\prime}\right)\left(\gamma^{\mu} \vec{p}_{*}^{\prime}+m^{\prime}\right)$ (1-eomponent form)

$$
\begin{aligned}
& \text { letling } \\
& p_{r}=p_{v}^{\prime}-p_{w} \\
& \|\left(o^{\mu}\right)_{\Delta \Delta p_{\mu}} \bar{\psi}_{+}^{\phi}(p, s)=m \psi_{-o}(p, o)
\end{aligned}
$$

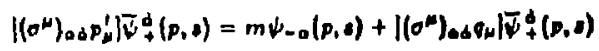

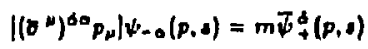

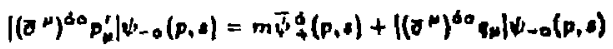

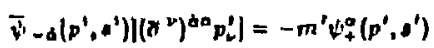

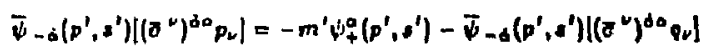

$$
\begin{aligned}
& \left.v q\left(p^{\prime}, q^{\prime}\right) \mid\left(\sigma^{*}\right)_{o \delta} p_{0}^{\prime}\right)=-m^{\prime} \bar{v}_{-\delta}\left(p^{\prime}, s^{\prime}\right)
\end{aligned}
$$

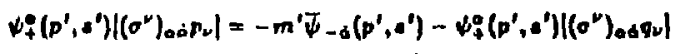

\section{B.2 QED Lagrangtay}

In four-component form

$$
\therefore: D D=i \bar{\psi} \tau^{\mu} \partial_{\mu} \psi-\epsilon \bar{\psi} \phi \psi-\frac{1}{4} F_{\mu \nu} F^{\mu \nu}-m \bar{\psi} \psi .
$$

Conside: $\cdots$, w

$$
\mathcal{C}_{K I N}=i\left\{\psi_{+}^{*}\left(0^{\mu}\right)_{\Lambda d} \partial_{\mu} \bar{\nabla}+\bar{\phi}_{-d}\left(\partial^{\mu}\right)^{\alpha 0} \partial_{\mu} \psi_{-0}\right\} \text {. }
$$

Now it is $\int \mathcal{A}_{2} \mathcal{L}$ which will be of intereat in a path integral 10 we may integrate the second term by parts. We s/so enticommute the apinors in the frat term to obtain (iznoring curtace terms)

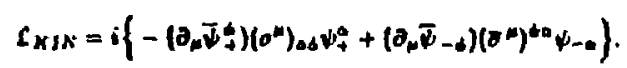

Now we lower the spinor indices with $\mathrm{o}_{0}$ and raise them on $\left(0^{\mu}\right)_{\text {do }}$ with eof to obtain

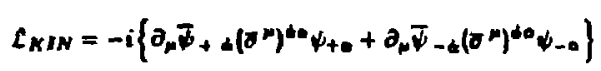

which is the form often found in the literalure.' Similarly the remainder of the Lagrangian is

$$
\begin{aligned}
& C_{Q E D}=C_{K I N}-c\left[\psi_{*}\left(0^{\mu}\right\}_{Q d} A_{\mu} \bar{\psi}_{+}^{+}+\bar{\psi}_{-\alpha}\left(\partial^{\mu}\right)^{d \alpha} A_{\mu} \psi_{-\alpha}\right] \\
& -\frac{1}{4} F_{\mu \nu} F^{\mu \nu}-m\left|\psi_{-a} \psi_{+}^{\alpha}+\bar{\psi}_{+}^{d} \bar{\psi}_{-d}\right|
\end{aligned}
$$

The Geld operatora of (B.s) thould be thought of an anihilating the eorresponding 6elds. Therefore, aince $\psi_{-}$is a tef-handed eleetron and $\psi_{\dagger}$ is a itfi-honded positron, $-m \psi_{-a} \psi_{f}$ destrojs an $\left(e^{-}\right)_{2}$ and an $\left(e^{+}\right)_{2}$, which is the came as destroying an $\left(e^{-}\right)_{L}$ and treating an $\left(e^{-}\right)_{R}$, with strength " $m^{n}$. 


\section{B.3 Chnality-Changho Opematoms}

The oparators which change one chiral atste to another are

Particle:

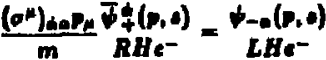

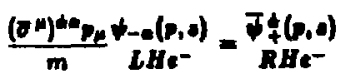

Antiparticles;

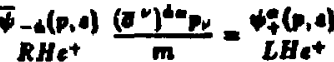

$$
\begin{aligned}
& \psi_{+}^{*}(p, s) \underline{\left(\sigma^{v}\right)_{a+p v}}=\bar{T} \underline{(p, s)} \\
& \mathrm{LHe}^{+} \frac{\mathrm{m}}{m}=\mathrm{RH}^{+}
\end{aligned}
$$

These relutions follow directly from the Dirue equation.

\section{B.4 Propagators}

We cen decompas the election Dirac propagatos into

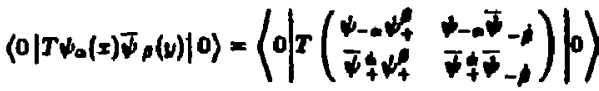

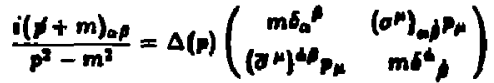

$$
\begin{aligned}
& \text { with } \quad \Delta(p) \equiv \frac{i}{p^{2}-m^{2}}
\end{aligned}
$$

which we can interpret a

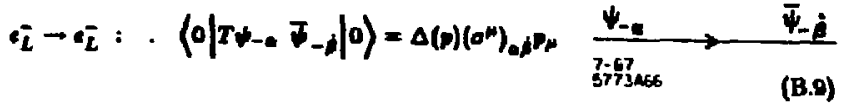

To get $e_{\bar{L}}^{-} \rightarrow e_{\bar{R}}^{-}$we apply the helicity-chunging operator $\left(\sigma^{\circ}\right)^{\mathrm{d} /} / \mathrm{m}$ to get

$$
\Delta(p)\left(a^{m}\right)_{a+p_{\mu}} \frac{\left(o^{\mu}\right)^{\mu} p_{\nu}}{m}
$$

But since $(\sigma \cdot p) a d(\sigma \cdot \sigma)^{\omega}=p^{2} s=m^{2} s t$ (on ahell) then

$$
e_{L}^{-} \rightarrow e_{R}^{-}: \quad(0|T+-\infty| 0)=m \Delta(0) \sigma_{a}^{0}
$$

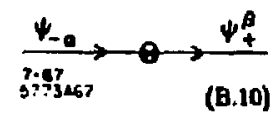

Which we could have fend of of (B.4) directy (O indicales helicity Bip). Similarly

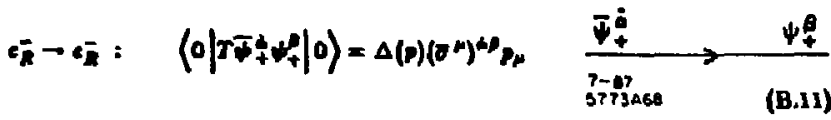

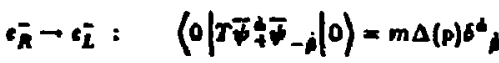

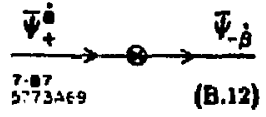

We may apply c's to these which will raise and lower indices as desired using the sules of Appendix A.3. For example, applying $\epsilon_{a}$ to (B.10) results in

$$
\left\langle 0\left|\psi_{-a} \psi_{+}\right| 0\right\rangle=-m \Delta(p) c_{\alpha} \rho
$$

In general the helicity-preserving operators are proportional to $\sigma^{\mu} p_{\mu}$ while the helicily-changing operators are proportional to $\mathrm{m}$. We do not consider multiple 
Aips since

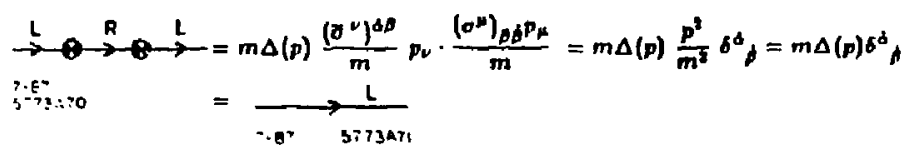

In come vense $\longrightarrow$ can be thought of as the appropriately normalized sum of

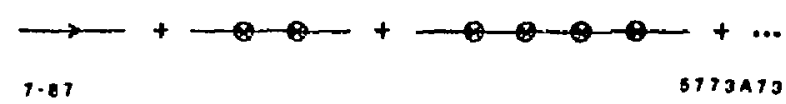

and $\rightarrow-Q \rightarrow-$ as the sum of

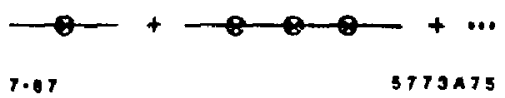

(normalized so that " $m$ " is the physical rouss).

Note that when externa) massive fermions are present in a process (i.e. the diegrams or Guen's fonctions have not been truncated) ouch felds moy change helicity in the legs of the diagram.

B.S PROJECTION OPERATORS

The analogue of the Dirac projection operatore for positive and negative energy stales

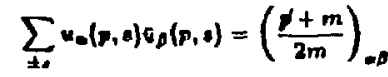

$$
\begin{aligned}
& \text { Ire: } \\
& \sum_{ \pm 0} \psi_{-\infty}(p, s) \bar{\psi}_{-j}(p, \Delta)=\frac{\left(o^{\mu}\right)_{a \phi} p_{\mu}}{2 m} \\
& \sum_{ \pm=} \bar{\psi}_{+}^{+}(p, 0) \psi_{+}^{\theta}(p, 0)=\frac{\left(\delta^{*}\right)^{d \theta} p_{\mu}}{2 m} \\
& \sum_{ \pm \infty} \psi_{-\infty}(p, \theta) \psi_{+}^{p}(p, \theta)=1 \delta_{0}^{\circ} \\
& \sum_{ \pm \theta} \bar{\psi}_{+}^{\delta}(p, \theta) \bar{\psi}_{-j}(p, \theta)=1 b^{d}
\end{aligned}
$$

In the case where spin be not summed over we project out both heliclty and eneres stales. In Dirac notation we have

$$
u_{0}\left(p_{1}\right) \sigma_{\beta}(p, v)=\left[\frac{p+m}{2 m} \frac{1+\eta s}{2}\right]_{O A}
$$

where $s^{\mu}=(0, \hat{s})$ in the rest frame. Let us consider the instance of a particle moving along the $+\vec{z}(3)$-exis very slowly $(\vec{p} \rightarrow 0)$ with spin in the $+\hat{z}$ dizection for right-handed particles. Then

$$
H_{L}=(0,0,0,1) \quad *_{R}^{*}=(0,0,0,-1) \text {. }
$$


For $t_{L}^{*}=(0,0,0,1)$ we find

$$
\begin{aligned}
& \psi_{-a}(p, s) \bar{\psi}_{-j}(p, \nu)=\frac{1}{4 m}\left[\left(\sigma^{\mu}\right)_{\alpha \phi} \nu_{\mu}+m\left(\sigma^{2}\right)_{a j}\right]
\end{aligned}
$$

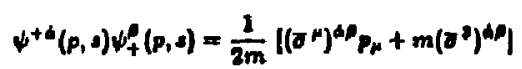

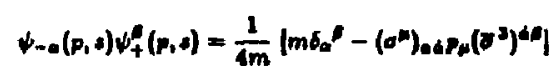

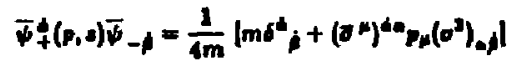

remembering that $\sigma^{2}=-\sigma^{2}$.

For photons

$$
\sum_{\lambda} C_{\mu}(t, \lambda) C_{\nu}(k, \lambda)=-g_{\mu \nu}
$$

\section{B.6 SIMPLE VERTICES}

Photon emission does not alp helicity (oince the current h trensvern). Thus the simple (truncated) vertices appear as
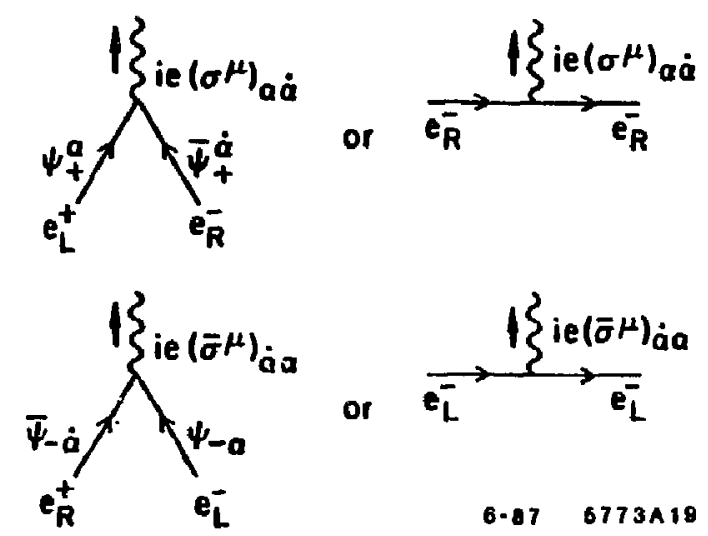


\section{APPENDIX C}

\section{Calculation of Anomalous Maznetic Moment I:}

$$
\text { Four-Component Spinors. }
$$

The standard calculation of the anomalous maznelic moment of the electron in lour-component spinor notation is presented here in detail. The rationale being that this is the protalype for all proceste considered in this paper. It provides a direct comperison with the two-component version which followa. We follow the approach of Rel. 2 but use the metric and opinor sepresentations of Appendix A

We examine the vertex correction

$$
\left.-i e \gamma_{\alpha p}^{\mu} \rightarrow-i e \Gamma_{\alpha \rho}^{\mu}\left(p, p^{\prime}\right)=-i e \mid \gamma^{*}+\Delta^{\mu}\left(p, p^{\prime}\right)\right)_{\alpha p}
$$

as illustraled in figuse 1 and whose lowert order contribution is given by the diagram in fgure 2. Since we will only be interested in the $1^{*}$ plece we will not dintingulsh between $\Gamma^{\mu}$ and $A^{*}$ but simply refer to the first order correction $a$ $\Gamma^{\mu}\left(p, p^{\prime}\right)$. To zuard against, o prioni, infrated divergence problems we will let the mass of the photon be $\mu$, which we shall lel go to zero at the end. We see that

$$
\begin{aligned}
& -i e \Gamma_{o s}^{\mu}\left(p, p^{\prime}\right)=(-i e)^{3} \int \frac{d^{4} k}{(2 \pi)^{4}}\left\{\left(\frac{-g_{o w}+\text { gauge terms }}{k^{2}-\mu^{2}+i c}\right)\left(\gamma^{*}\right)_{o r}\right.
\end{aligned}
$$

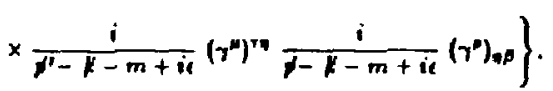

We will do away with the couge terms in the photon by recognizing that such terms vanish in Feynman zauge when $\boldsymbol{B} \rightarrow \mathbf{0}$. Hence

$$
\begin{aligned}
& \Gamma^{\mu}\left(p, p^{\prime}\right)_{\alpha p}=(-i \varepsilon)^{2} \int \frac{d^{\prime} k}{(2 \pi)^{\mu}}\left\{\left(\frac{-1}{k^{2}-\mu^{2}+i c}\right)\right. \\
& \times\left[\gamma^{\nu} \frac{i\left(p^{\prime}-k+m\right)}{\left(p^{\prime}-k\right)^{2}-m^{2}+i \epsilon} \gamma^{\prime \prime} \frac{i(\nu-k+m)}{(p-k)^{2}-m^{2}+i t} \gamma_{\alpha \beta}\right\}
\end{aligned}
$$

We will evaluate this on-shell where $f=f^{\prime}=m$ and then let $q^{2} \rightarrow 0$, i.e. $p^{\prime} \rightarrow p$ We ignore the other eraphe which occur at this order st they only contribute to the self-energy renormalizations. In order to regulate the integral we eroploy the traditional Pauli-Villars" eubtraction scheme. This introduces a "masgive photon" of mass $A \rightarrow \infty$ which utilizes an incorrect metric. The "incorrect metric $^{n}$ in this case is the negative of the one given in aection A.2. The result is that the heary photon propazator olways appears with sizn opposite to that of the light $(\mu \rightarrow 0)$ propagator. Thus the lending divercences cancel. Although such a propagator is not phyrically meaninzful, as we take $A \rightarrow \infty$ such cantributions vanish. Such a scherne is not overtly gauge inveriant during the intermediale steps of the calculations but, of courat, gauge inveriance must be reatosed once the limit is taken. Consequently we may represent the combined photon propagators by

$$
\frac{1}{k^{2}-\mu^{2}}-\frac{1}{k^{2}-A^{3}}=-\int_{\mu^{1}}^{\mu^{3}} \frac{d t}{\left(k^{2}-t\right)^{2}}
$$

resulting in 


$$
\begin{aligned}
& r^{\mu}\left(p, p^{\prime}\right)=-i e^{2} \int \frac{d k}{(2 x)^{a}} \int_{m}^{N}-d t \frac{N *}{D} \\
& N^{\mu}=\gamma^{\mu}\left|i\left(\mu^{\prime}-k+m\right)\right| \gamma \gamma^{\mu}|i(\gamma-k+m)| \gamma r \\
& D=\left(k^{2}-t^{2}\right)^{3}\left(\left|p^{\prime}-k\right|^{2}-m^{2}\right)\left(|p-k|^{3}-m^{2} \mid\right. \text {. }
\end{aligned}
$$

Now using the standard gamma matrix contraction identities of the form $\gamma^{2}|\cdots| \gamma_{\nu}$ which can be found in the appendices of Bjorken and Deal ${ }^{\mathbf{H}}$ we find that

$$
\begin{aligned}
& N^{\mu}=i^{2}\left\{-2(\gamma-k) \gamma_{\mu}\left(r^{\prime}-k\right)-2 m^{2} \gamma^{\mu}+2 m\left(\left(\gamma^{\prime}-k\right) \gamma^{2}+\gamma^{\prime \prime}\left(\gamma^{\prime \prime}+k\right)\right.\right. \\
& \left.\left.+(\mu-k) \gamma^{2}+\eta^{\mu}(x-k)\right)\right\} \\
& =2\left\{(p-k) \tau^{\mu}\left(p^{\prime}-k\right)+m^{2} \gamma^{\mu}-m\left[\tau^{\mu}\left(\gamma^{\prime}+\not-2 k\right)+\left(p^{\prime}+\gamma-2 k\right) \gamma^{\prime \prime} \mid\right\}\right. \\
& \text { (C.6) }
\end{aligned}
$$

where we have used $\left\{\gamma^{\mu}, \gamma^{\nu}\right\}=2 g^{\mu \nu}$ and therefore $2 p^{\mu}=\gamma^{\mu}+\gamma^{\mu} \gamma$. Since $p^{2}=m^{2}$ on- hell we have $(p+k)^{3}-m^{2}=k^{2}-2 p \cdot k$ and -00

$$
D=\left(k^{2}-t^{2}\right)^{2}\left(k^{2}-2 p^{\prime} \cdot k\right)\left(k^{2}-2 p \cdot k\right)
$$

Using Feynman's trick

$$
\frac{1}{a^{2} b c}=6 \int_{0}^{1} d z \int_{0}^{1-z} d y \frac{1-x-y}{\mid a+(b-0) x+(c-a) y]^{a}}
$$

(c.8)

from (H.S) to give

$$
\frac{1}{D}=6 \int_{0}^{1} d x \int_{0}^{1} d y \int_{y^{2}}^{k^{2}} d t \frac{(1-x-y)}{\left[k^{2}-t-(2 p \cdot k-t) x-\left(2 p^{2} \cdot k-t\right) y\right]^{4}} .
$$

As
Now use (H.7) so evaluate this realizing that

$$
\operatorname{lop} \rightarrow p^{\prime} y, \quad:=-t+t x+t y=-t(1-x-y)
$$

to yield

$$
\int \frac{d k}{(2 \pi)^{4}} \frac{N^{\mu}}{D}=6 \int_{0}^{1} d x \int_{0}^{1-z} d y \int_{\mu^{3}}^{L^{3}} d \frac{i M^{\mu}}{96 x^{2}\left[-l(1-x-y)-p x+p^{2} y\right)^{2}}
$$

with

$$
\begin{aligned}
& M^{\mu}=2 f^{\mu} x^{\prime}-2\left|f^{\mu}\left(f x+f^{\prime \prime} y\right)+\left(f^{\prime}+f^{\prime} y\right) \gamma^{\mu} x^{\prime \prime}\right| \\
& -2 m^{2} \gamma^{\mu}+\left|-t(2-x-y)-\left(\gamma x+\gamma^{\prime} y\right)^{2}\right| \gamma^{2} \gamma^{\mu} \gamma_{y}
\end{aligned}
$$

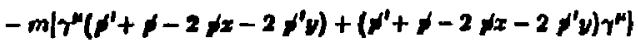

$$
\begin{aligned}
& \left.+2\left(y x+x^{\prime} y\right)\right)^{\prime \prime}\left(y x+y^{\prime} y\right)
\end{aligned}
$$

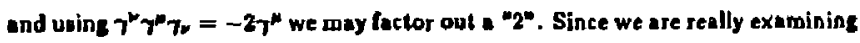
$q\left(p^{\prime}\right) r^{\prime \prime}\left(p, p^{\prime}\right) u(p)$ we may use the Dirac equation

$$
a\left(p^{\prime}\right)\left(p^{\prime}-m\right)=(\vec{p}-m) u(p)=0 \text { with } p^{\prime}=p+q
$$


on $A^{\prime \prime}$. Further, from $\left(11.17\right.$ b) $\left(p x+p^{\prime} y\right)^{2}=m^{2}(x+y)^{2}-q^{2} x y$ so that

$$
\begin{aligned}
& M^{\mu}=2\left\{\left(m-n \gamma^{\mu}\left(m+n+(\mid m-n x+m y) \gamma^{\mu}(m=+\{m+n v)\right.\right.\right. \\
& -\left(\mid\left(m-n x+m y \mid \gamma^{\mu}\left(m+n+\left(m-n \gamma^{m} \mid m x+(m+n y \mid)\right.\right.\right.\right. \\
& -m\left(\gamma^{2}(|m+n+m-2 m x-2| m+\Lambda v)\right. \\
& +\left(m+[m-q]-2[m-A \mid x-2 m v) \gamma^{m}\right] \\
& \left.+\left[1(1-x-y)+\left(m^{2}|x+y|^{2}-q^{2} x y\right)\right] \gamma^{*}-m^{2} \gamma^{m}\right] \\
& =2\left\{r^{4}(1)(2-x-y)+m^{2}(x+y)^{2}-y^{2} x y+m^{2}-2 m^{3}(x+y)\right. \\
& \left.-4 m^{2}(1-x-y)+m^{2}(x+y)^{2}-m^{2}\right) \\
& +\gamma^{\mu}(m-m(x+y)-m y-m+2 m y+m(x+y) v) \\
& +|-m+m x+m(x+y)+m-2 m x-m(x+y)| x \mid \\
& +\left\{n^{*} 1-1-x-y-x y\right\} \text {. }
\end{aligned}
$$

We have exercised our gauge freedom when we chore to calculate in the Feynman sauce. This is one of the $R_{l}$ (or Lorentz) rouges which we covariant, ie. satisfy $q^{u} A_{\nu}\left(q^{2}\right)=0$ (in this case $\xi=1$ ). In a physical process we have terms like $\Gamma^{\mu} A_{\omega}$

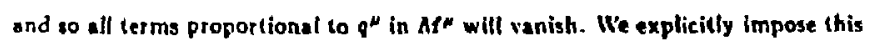
gauge condition: $Q^{\nu} A_{M}(q)=0$. Now

$$
\left\{\gamma^{\mu}, \gamma^{\nu}\right\}=2 g^{\mu \nu}
$$

thus

$$
\left\{\gamma^{\mu}, t\right\}=\left\{\gamma^{\mu}, \gamma^{\nu} \phi_{\nu}\right\}=2 \sigma^{\mu \nu} q_{\nu}=2 q^{\mu}
$$

17 which vanishes in $M^{r}$. Therefore

(C.14)

and the anti-commutstion term does not contribute. Also

$$
h \omega=-\gamma^{m} d t=-\gamma^{m} q^{2}
$$

Letting

$$
\begin{aligned}
A= & 2\left\{(1-x-y)+2 m^{2}(\mid x+y\}^{2}-2|1-x-y|-|x+y|\right) \\
& \left.-q^{2} x y-y^{2}(1-x)(z-y)\right\}
\end{aligned}
$$

(C.13) becomes

$$
\begin{aligned}
& M^{\mu}=\gamma^{H}\{A\} \\
& \left.\left.+\left\{m \gamma^{*}(x+y)-x(x+y)-x\right)-m 7^{\mu} f(x+y)-y(x+y)-y\right)\right\} \\
& =y^{\prime \prime}(A)+2 m\left(\left|T^{\prime \prime}\right|-x^{2}-x y+y\right)-7^{\prime \prime}\left(1-y^{3}-x y+x\right) \\
& =\gamma^{\mu}(A) \\
& +2 m\left\{d y^{y}\left[-\frac{x^{3}}{2}-x y+\frac{y^{2}}{2}+\frac{x}{2}-\frac{x^{2}}{2}+\left(-\frac{x^{2}}{2}-\frac{x}{2}+\frac{y^{2}}{2}+\frac{y}{2}\right)\right]\right\} \\
& \left.-\gamma\left(-\frac{y^{2}}{2}-x \dot{x}+\frac{x}{2}+\frac{y}{2}-\frac{x^{2}}{2}+\left(-\frac{y^{2}}{2}-\frac{y}{2}+\frac{x^{2}}{2}+\frac{y}{2}\right)\right]\right\}
\end{aligned}
$$

but

$$
\gamma^{\mu}\left(-\frac{x^{2}}{2}-\frac{x}{2}+\frac{y^{2}}{2}+\frac{y}{2}\right)-\gamma^{\mu}\left(-\frac{y^{2}}{2}-\frac{y}{2}+\frac{x^{2}}{2}+\frac{x}{2}\right)=0
$$


by (C.14) thu e

$$
\begin{aligned}
M^{\mu} & =\gamma^{\mu}(\Lambda)+\frac{f^{\mu}-\gamma^{\mu}[}{2}\left[2 m\left(x+y-x^{2}-y^{2}-2 x y\right)\right] \\
& =\gamma^{\mu}(\Lambda)+\frac{\left|\gamma^{\mu}, \gamma^{\mu}\right| q y}{2}[2 m(x+y)(2-x-y)]
\end{aligned}
$$

with

$$
\Gamma^{\mu}\left(p, p^{\prime}\right)=0 \int_{0}^{1} d x \int_{0}^{1-3} d y \frac{-1}{\partial 6 \pi^{2}}\left(i \varepsilon^{2}\right) \int_{\mu^{2}}^{A^{2}}(-d t) \frac{i(1-x-y) M^{\mu}}{\left[t(1-x-y)+\left.(p x+p y)^{2}\right|^{2}\right.} .
$$

Now

$$
a^{\mu \nu}=\frac{i}{2}\left[\gamma^{\mu}, \gamma^{\mu}\right]
$$

and

$$
\Gamma^{\mu}\left(p, p^{\mu}\right)=\tau^{\mu} F_{1}\left(q^{2}\right)+\frac{i}{2 m} a^{\mu \nu} q_{w} F_{2}\left(q^{2}\right)
$$

with the anomalous magnetic moment

$$
a_{2} \equiv F_{2}\left(\varphi^{2} \rightarrow 0\right) \quad a \equiv \frac{-2}{2}
$$

Therefore

$$
\begin{aligned}
i F_{2}\left(p^{2} \rightarrow 0\right) & =\frac{2}{i} \cdot \frac{-6(i)^{2} e^{2}}{-96 \pi^{2}} \int_{0}^{1} d x \int_{0}^{1-2} d y \int_{\mu^{1}}^{L^{2}} d t \frac{(1-x-y) 2 m^{2}(x+y)(1-x-y)}{\left(1(1-x-y)+m^{2}(x-y)^{2}\right]^{2}} \\
i a_{c} & =-i \frac{a m^{2}}{x} \int_{0}^{1} d x \int_{0}^{1-2} d y \int_{\mu^{1}}^{\mu^{4}} d t \frac{(x+y)(1-x-y)^{2}}{\left[(1-x-y)+\left.m^{2}(x-y)^{2}\right|^{2}\right.}
\end{aligned}
$$

where $a \equiv c^{2} / 4 \pi$. Performing the Reintegration

$$
\begin{aligned}
i e_{4}=- & -i \frac{a m^{2}}{x} \int_{0}^{1} d x \int_{0}^{1-z} d y \frac{(x+y)(1-x-y)^{2}}{(1-x-y)} \\
& \times\left\{\frac{1}{\mu^{2}(1-x-y)+m^{2}(x-y)^{2}}-\frac{1}{\mu^{2}(1-x-y)+m^{2}(x-y)^{2}}\right\}
\end{aligned}
$$

and taking $\Delta \rightarrow \infty$

$$
i c_{8}=i \frac{a}{y} \int_{0}^{1} d x \int_{0}^{1-x} d y \frac{(x+y)(1-x-y)}{\left(\mu^{2} / m^{2}\right)(1-x-y)+(x-y)^{2}}
$$

Let $x=x+y$

$$
c=\frac{\alpha}{a} \int_{0}^{1} d x \int_{z}^{1} d x \frac{z(1-x)}{\left(\mu^{2} / m^{2}\right)(1-x)+z^{2}}
$$

and we see that this remalas finite where $\mu^{2}=$ mess of photon $\rightarrow 0$. Doing this

$$
\begin{aligned}
e_{a} & =\frac{a}{x} \int_{0}^{1} d x \int_{0}^{1} d x \frac{1-x}{x} \\
& =\left.\frac{a}{\pi} \int_{0}^{1} d x|\ln z-x|\right|_{x} ^{1} \\
& =\frac{a}{x} \int_{0}^{1} d x|-\ln x-1+x| \\
& =\left.\frac{a}{\pi}\left|-x \ln x_{x}-x+\frac{x^{2}}{2}\right|\right|_{0} ^{1} \\
& =\frac{a}{2 \pi}
\end{aligned}
$$

so 
Thus we have found

\section{FIGURE CAPTIONS}

$$
a_{x}=\frac{a}{2 x}
$$

(C.)7) 1. Vertex correction

which it the Schwinger result.

$$
-i e T_{a s}^{\mu} \rightarrow-i e \Gamma_{a, p}^{\prime \prime}\left(p, p^{\prime}\right)=-i e\left(T^{\prime \prime}+\Delta^{\mu}\left[p, p^{\prime}\right)\right\}_{O A}
$$

2. Lowest ordes contribution ta the vertex correction 


\section{APPENDIX D}
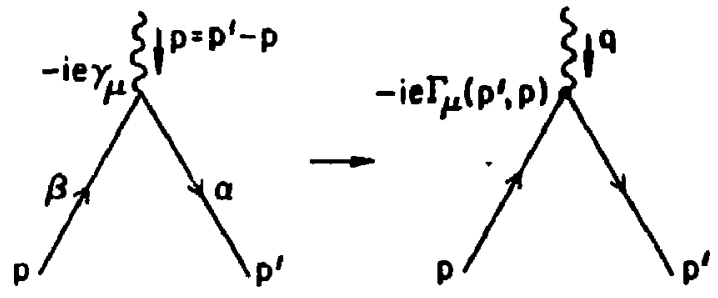

Fig. C.1

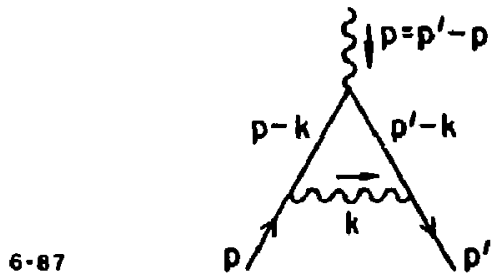

Fig. C.2

\section{Cakulation of Anomabus Mazactic Moment I}

Two-Component Spinors.

We repeat the cakculation performed in Appendix $\mathrm{C}$ but employ two-component Weyl spinors in order to illustrate their use. We will specifically examine the verlex correction to

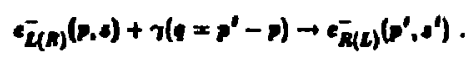

Certain commente should be made. The procesen $e_{\bar{L}}^{-}+\gamma \rightarrow e_{\bar{R}}^{-}$and $\bar{c}_{\bar{R}}+\gamma \rightarrow \epsilon_{\bar{L}}$ are expected to provide equivaleatly to the anomelous moment a. The proceasea $e_{L} \rightarrow e_{L}$ and $e_{2} \rightarrow e_{2}$ contribute solely to the verlex (wavefunction) renormal.

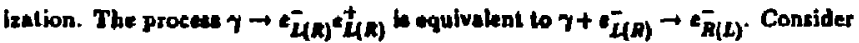

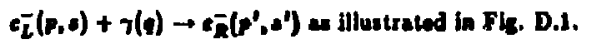

We would nalvely consider these to be the sole contributions. To see that this does not lead to the correct result we procesed to evaluate them. Remembering from Eqn. (C.1) that the vertex is given by $\mu^{\mu}=-i e \psi_{+} \Gamma^{\mu} \psi_{-}$we find:

Diazram I:

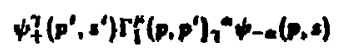

$$
\begin{aligned}
& =i c^{2} \int \frac{d^{4} k}{(2 x)^{4}} m \Delta(p-k) \Delta\left(r^{\prime}-k\right) \frac{1}{k^{2}}
\end{aligned}
$$

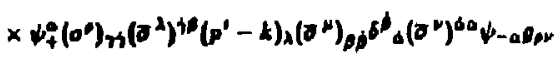

$$
\begin{aligned}
& =i e^{2} \int \frac{N^{4} t}{(2 x)^{4}} \frac{N_{f}^{n}}{D}
\end{aligned}
$$


where we have introduced the notalion

$$
\Delta(p) \equiv i / p^{2}-m^{2}+i c
$$

we find that

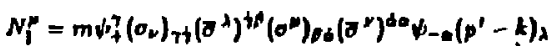

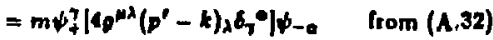

$$
\begin{aligned}
& =\operatorname{lm}\left(p^{\prime}-k\right)^{\mu} \psi_{+}^{0} \psi_{-}
\end{aligned}
$$

Diagram 1:

$\psi_{+}^{\top}\left(p^{\prime}, s^{\prime}\right) r_{*}^{\mu}\left(p, p^{\prime}\right) \varphi^{0} \psi_{-\infty}(p, s)$

$$
\begin{aligned}
& =i e^{3} \int \frac{d^{d} k}{(2 \pi)^{4}} m \Delta(p-k) \Delta\left(p^{\prime}-k\right) \frac{1}{k^{3}}
\end{aligned}
$$

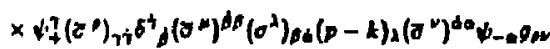

$$
\begin{aligned}
& =i e^{2} \int \frac{d^{4} k}{(2 \pi)^{4}} \frac{N_{i}^{\mu}}{D}
\end{aligned}
$$

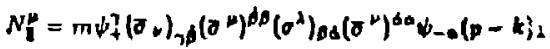

$$
\begin{aligned}
& =m \psi_{+}^{2} \mid\left\langle g^{\mu \lambda}(p-k)_{\lambda} \delta_{\gamma}^{*}\right| \psi \ldots \\
& =4 m(p-k)^{\mu} \psi_{+}^{a} v_{-a}
\end{aligned}
$$

and, as in Eqn. (C.7),

$$
D=k^{2}\left(k^{2}-2 p \cdot k\right)\left(k^{2}-2 p^{\prime} \cdot k\right) \text {. }
$$

The total is given by

$$
\begin{aligned}
& v_{+}^{7} \Gamma^{\mu}\left(p, p^{\prime}\right)_{1}^{a} \psi_{-0}=i e^{2} \int \frac{d^{\mu} k}{(2 \pi)^{4}} \frac{N^{\mu}}{D} \quad \text { with } \\
& N^{\mu}=N_{f}^{\mu}+N_{f}^{\mu}=4 m \psi q\left(p^{\prime}, \varepsilon^{\prime}\right)\left|\left(p^{\prime}-k\right)^{\mu}+(p-k)^{\mu}\right| \psi_{-a}(p, s) \text {. }
\end{aligned}
$$

As in Appendix C (C.4) we could use a Paull-Villars regulator $(\Lambda \rightarrow \infty)$ and let the photon acquire a smalt mass $(\mu \rightarrow 0)$ so that

$$
\frac{1}{k^{3}} \rightarrow-\int_{n^{2}}^{\lambda^{2}} d t \frac{d t}{\left(k^{2}-t^{2}\right)^{2}}
$$

and

$$
D \rightarrow\left(k^{2}-i^{2}\right)^{2}\left(k^{2}-2 p^{2} \cdot k\right)\left(k^{3}-2 p \cdot k\right)
$$

$$
\int \frac{d^{4} k}{(2 \pi)^{4}} \rightarrow-\int \frac{d^{4} k}{(2 \pi)^{4}} \int_{u^{2}}^{d^{3}} d t \text {. }
$$

Then using (C.8)

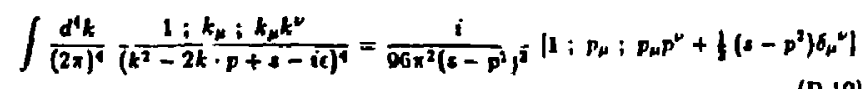

from (H.7) with $s=-t(1-x-y)$ and $p=p z+p^{\prime} y$. We have seen in Appendix $\mathrm{C}$ that this was unnecessary since, as we had argued from general principles, the helicity-ehanging part is finite. Thus we ust instead

$$
\frac{1}{a b c}=2 \int_{0}^{1} d x \int_{0}^{2-x} d y \frac{1}{[a+(b-a) x+(c-a) y]^{3}}
$$

and

$$
\int \frac{d^{\prime} k}{(2 \pi)^{4}} \frac{1 ; k_{\mu}}{\left(k^{2}-2 k \cdot p+-i\right)^{3}}=\frac{i\left|1 ; p_{\mu}\right|}{32 \pi^{2}\left(s-p^{2}\right)}
$$


froin (H.6). Unins (D.11) and (D.6) yields

$$
\frac{1}{D}=2 \int_{0}^{1} d x \int_{0}^{1-2} d y \frac{1}{\left[k^{2}-2 p \cdot k x-2 p|\cdot k y|^{2}\right.}
$$

Note, however, that we can equally wall interchange " $" *$ and "c* in $(0,11)$ (as outlined in (H.20)) in which care (D.13) would have become

$$
\frac{1}{D}=2 \int_{0}^{1} d x \int_{0}^{1-y} d y \frac{1}{\left[k^{3}-3 p^{2} \cdot k x-3 p \cdot k y\right]^{2}}
$$

Wo see that the net result is to interchange $x$ and $y$ in the integrand. Ualns the notation of (D.12) we see, in the caes of (D.19), that p $\rightarrow$ px + p'y while for (D.14) $p \rightarrow p y+p^{\prime} x$, In both cases $\bullet=0$.

Usine (D.12) and (D.7) [with (D.13) beine used for $1 / D \mid$ rields

$$
\psi_{+} \Gamma^{\mu}\left(p, \rho^{\prime}\right) \psi_{-}=2 i e^{*} \int_{0}^{1} d x \int_{0}^{1-2} d y \frac{-i}{32 \pi^{2}} \frac{\mu^{\mu}}{\left(p x+\nabla^{\prime} y\right)^{2}}
$$

end, acain, from (H.17 b) $\left(p x+p^{\prime} y\right)^{2}=m^{2}(x+y)^{2}-p^{2} x y$ where $e=p^{\prime}-p$.

$$
M^{\mu}=4 m \psi_{+}\left(p^{\prime}\right)\left|p^{\prime \prime}(1-2 x)+p^{\prime \prime}(1-2 y)\right| \psi-(p)
$$

thus

$$
\psi_{+} \Gamma^{\mu}\left(p, p^{\prime}\right) \psi_{-}=\frac{e^{2}}{16 x^{2}} \int_{0}^{1} d x \int_{0}^{1-x} d y \frac{4 m \psi_{+}\left|p^{\prime \prime}(1-2 x)+p^{2} p(1-2 y)\right| \psi}{m^{2}(x+y)^{2}-\tau^{2} x y} \text {. }
$$

Now il we had used (D.14) inslead of (D.13) (i.e. Kt "b $\rightarrow 4^{*}$ ) then we muat ixterchante $x$ and $y$ in the intesrand to sot

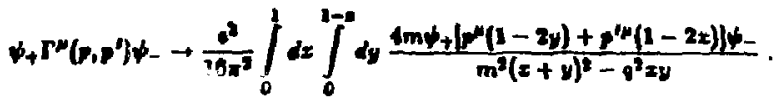

Nole that the denorainctor to the wask. We may now average there two expressione to oblaln

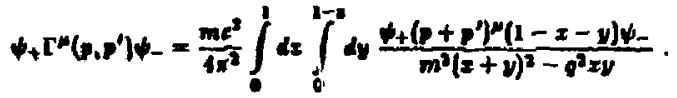

Thi procedure of witchlar " $t$ " and "c* in exprassions like (D.11) and aver-

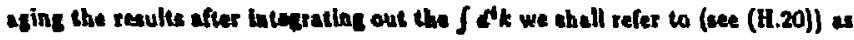
the "bt$\rightarrow c$ trick", It is quits general and can be aleo ueed in the regulated vertion presented in Appendix $C$. The result is genurally a simple funclion of $x+y$ and. $x-y$ (cubthatios arie orghal).

The exprescion is (D.10) represents one half of the anomaly (the $e_{L} \rightarrow t_{R}$ part). We may similarly compula the other $\left(c_{n}-e_{L}\right)$ part. We find that if is the wine as (D.10) except that

$$
\phi_{+}^{*}\left(p^{\prime}\right)+-\infty(p) \rightarrow \bar{\phi}_{-\alpha}\left(p^{\prime}\right) \bar{\psi}+(p)
$$

Thls sum ean be ropresented by

$$
\frac{m e^{2}}{4 \pi^{3}} \int_{0}^{1} d x \int_{0}^{1} d y \frac{M m_{u m}}{m^{2}(x+y)^{2}-q^{2} x y}
$$

wibere

$$
M_{s m m}^{*}=(1-x-v)\left(\psi_{+}\left(p^{\prime}\right) \phi_{-}(p)+\bar{\psi}-\left(p^{\prime}\right) \bar{\psi}+(p)\right)\left(p+p^{\prime}\right)^{\mu}
$$

Now we may employ the geaeralized Gordon identity of Appendix H (Egn. H.13) 
which states

$\left(\psi+\left(p^{\prime}\right) \psi-(p)+\bar{\psi}-\left(p^{\prime}\right) \bar{\psi}+(p)\right\}\left(p+p^{\prime}\right)^{\mu}=$

$$
-i\left|\psi_{+}\left(p^{\prime}\right) a^{\mu \nu} q u \psi_{-}(p)+\bar{\psi}-\left(p^{\prime}\right) a^{w v} q u \psi_{+}(p)\right|
$$

$+2 m\left[\bar{\psi}-\left(p^{\prime}\right) \sigma^{\mu \nu} \bar{\psi}_{+}(p)+\psi_{+}\left(p^{\prime}\right) \sigma^{\mu \nu} \psi-(p)\right]$

Using this in (D.22) we may ignore the find tetm a it contributes solely to the vertex renormalization. Thus (D.22) may be written as

$$
\left.\Gamma^{\mu}\left(p, p^{\prime}\right)=\frac{-i m e^{2}}{4 t^{2}} \mid \psi_{+}\left(p^{\prime}\right) \sigma^{\mu \nu} q_{v} \psi_{-}^{*}(p)+\bar{V}_{-}\left(p^{\prime}\right) \sigma^{\mu \nu} q_{w} \psi_{-}(p)\right] J
$$

where

$$
I\left(q^{2}\right)=\int_{0}^{1} d x \int_{0}^{1-x} d y \frac{(1-x-y)}{m^{2}(x+y)^{2}-q^{2} x y} .
$$

From (A.8) we see that this can be written fn four-component Dirac form as

$$
\bar{\psi}(p) T^{\mu}\left(p, p^{*}\right) \psi(p)=\frac{-i m a l\left(q^{2}\right)}{\pi} \bar{\psi}\left(p^{\prime}\right) a^{\mu \nu} q, \psi(p),
$$

where we have used $a=c^{2} / 4 \pi$, or simply

$$
\Gamma^{\mu}\left(p, p^{\prime}\right)=\frac{-i m \alpha I\left(q^{2}\right)}{\pi} \sigma^{\mu \nu} q_{*},
$$

From (C.15) this implies

$$
F_{2}\left(q^{2}\right)=-\frac{2 m^{2} \alpha J\left(q^{2}\right)}{\pi}
$$

and from (C.16)

$$
a_{a}=-\frac{2 m^{2} \alpha I(0)}{\pi}
$$

From (D.24)

$$
I(0)=\int_{0}^{1} d x \int_{0}^{1-x} d y \frac{(1-x-y)}{m^{2}(x+y)^{2}}
$$

usine (H.10 c)

$$
\int_{0}^{1} d x \int_{0}^{1-2} d y=1 \int_{0}^{1} d x \int_{-1}^{1} d x
$$

therefore

$$
I(0)=\frac{1}{m^{2}} \int_{0}^{1} d z \int_{0}^{1} d z \frac{1-z}{z^{2}}=\frac{1}{m^{2}} \int_{0}^{1} d z \frac{1-z}{z}
$$

Hence

$$
a_{x}=-\frac{2 a}{\pi} \int_{0}^{1} d x\left(1-\frac{1}{x}\right)
$$

which is divergent!

The reason that the naive contributions do not reproduce the Schwinger result is that the $F_{1}$ and $F_{2}$ parts of $\Gamma^{*}$ are intimately tied together for masgive spinor. This is manitestly evident in the Gordon decomposition (Appendix $H$ ). In (D.23) we say that we senerated . " $\gamma^{\mu}$ " type term which is indicalive of - helicity-preserving vertex. This is in spite of the fact that our diagrammatic representations in Fis. D.1 contained explicil helicily fips (indicated by 8 ). Similarly = $\sigma^{\mu \nu}=$ type terms may be zenerated from the diagrams connecting $e_{L}$ to $e_{L}$ and $e_{R}$ to $e_{R}$. Carefully following the derivation in Appendix $C$ we eee that this is indeed the case. There we start and end with $\bar{\psi}$ and $\psi$ which contain both left and right-handed parta. To take this over to the two-component ease we would have to add the four amplitudes for $\ell_{L}$ or $R+\gamma \rightarrow e_{L}$ or $R$ together. 
This is somohow disteleful. We would like to be able lo start with a apacitad thete, ay $c_{L}$, and end with $c_{R}$ without reference to additionat procenem. One can obscure this disagreeable situntion by penmitiog the axterat lege wo be chitulity-changins. This is nat an option for truacated distams but we really

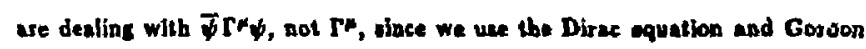
identitien.

Adopting thix approuch we have eix additional dingrame which should be added to dingrams $I$ and $B$ in Fit. D.I. These are found in Pig. D.z.

Following the anme procedure as before but rezulatiog the integral as in Appendix $\mathrm{C}($ see $(C .1))$ we find

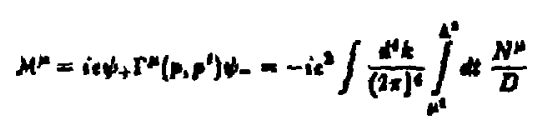

with " $D^{n}$ as is $(D .9)$ and

$$
\begin{aligned}
& N^{\mu}=\psi_{+}(p)\left\{\frac{\sigma \cdot p^{\alpha}}{m} \sigma^{\mu} m \sigma^{\mu a} \cdot(p-t) \sigma_{\mu} \frac{\partial \cdot p}{m}\right.
\end{aligned}
$$

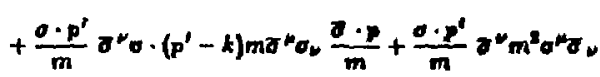

$$
\begin{aligned}
& +\frac{\sigma \cdot p^{\prime}}{m} \partial^{2} \sigma \cdot\left(p^{\prime}-k\right) \sigma^{\mu} \sigma \cdot(p-k) \sigma_{\nu}+a^{2} \sigma \cdot\left(p^{\prime}-k\right) m a^{\mu} \sigma_{\nu} \\
& +a^{\prime \prime} m \sigma^{\mu} \sigma \cdot(p-2) \sigma
\end{aligned}
$$

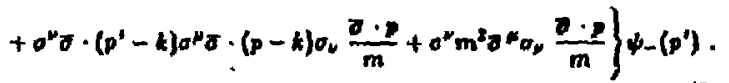

\section{We make wes of the relations of Section (A.B) to obtain}

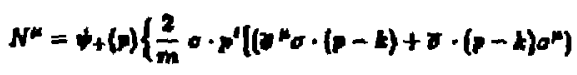

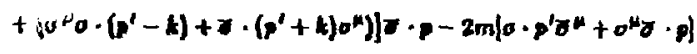

$$
\begin{aligned}
& -\frac{2}{m}\left(\sigma \cdot p^{\prime} z \cdot(p-k) \sigma^{\prime} \sigma \cdot\left(p^{\prime}-k\right)+\sigma \cdot(p-k) \sigma^{\mu} \sigma \cdot\left(p^{\prime}-k\right) a \cdot p\right) \\
& +2 m\left(0 \cdot\left(b^{\prime}-4\right) \sigma^{\prime}-a^{m} \cdot\left(b^{\prime}-4\right)\right. \\
& \left.\left.+a \cdot(p-t) z^{n}+a^{4} z \cdot\left(b^{\prime}-t\right)\right]\right)+-\left(p^{\prime}\right)
\end{aligned}
$$

Performiag the integrals os in (C.O) (but Gnoting the f-term at if contribulen colely to renormalization) and using (C.U)

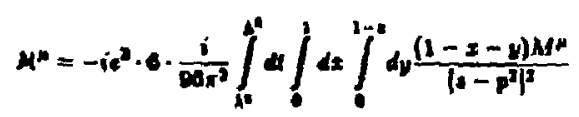

where

$$
1-p^{2}=-\left[u(1-z-y)+\left(p x+p^{\prime} v\right)\right]^{2}
$$

and $M^{\mu}$ is an abreviztion for the expresion 


$$
\begin{aligned}
& M^{\prime \prime}=\psi_{+}\left(p^{\prime}\right)\left\{\frac { 2 } { m } 0 \cdot p ^ { \prime } \left(z w_{0} \cdot\left(p+p^{\prime}-2 p x-2 p^{\prime} y\right)\right.\right. \\
& \left.+z \cdot\left(p+p^{\prime}-2 p x-3 p^{\prime} y\right) p^{\prime}\right] d \cdot p
\end{aligned}
$$

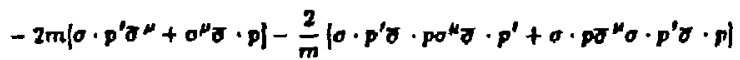

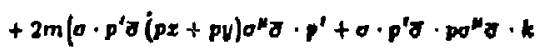

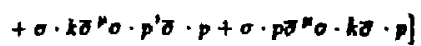

$$
\begin{aligned}
& -2 m\left[0 \cdot p^{\prime} \bar{\delta} \cdot\left(p z+p^{\prime} y\right) \alpha^{\prime \prime} \partial \cdot\left(p x+p^{\prime} y\right)+\sigma^{\prime \prime} \sigma \cdot\left(p x+p^{\prime} y\right)\right. \\
& \left.+\sigma \cdot\left(p x+p^{\prime} y\right) \partial \partial^{N} \sigma \cdot\left(p x+p^{\prime} y\right) \delta \cdot p\right] \\
& \begin{array}{r}
\left.+2 m\left\{\sigma \cdot\left(p+p^{\prime}-2 p x-2 p y\right) \sigma^{\prime \prime}+\sigma^{\mu} \sigma \cdot\left(p+p^{\prime}-2 p x-2 p^{\prime} y\right)\right]\right\} \\
\text { (D- }-(p)
\end{array}
\end{aligned}
$$

Now use Eqn. (B.2):

$$
\bar{v}+(p)=(\bar{a} \cdot p / m) w_{-}(p)
$$

ind

$$
\bar{\psi}-\left(p^{\prime}\right)=\psi_{+}\left(p^{\prime}\right)\left(\sigma \cdot p^{\prime} / m\right)
$$

Ilone with $q=p^{t}-p$ to find

$$
\begin{aligned}
& M^{H}=\bar{\psi}-\left(p^{\prime}\right)\left\{2 m \mid \bar{\sigma}^{\mu_{O}} \cdot\left(p+p^{\prime}-2 p x-2 p^{\prime} y\right)\right. \\
& \left.\left.+\sigma \cdot\left(p+p^{\prime}-2 p z-2 p^{\prime} y\right) \sigma^{\mu}\right]\right\} \bar{\psi}+(p) \\
& -\bar{\psi}-\left(p^{\prime}\right)\left|2 m^{2} \partial^{\mu}\right| \psi-(p)-\psi+\left(p^{\prime}\right)\left|\sigma^{*} 2 m^{2}\right| \bar{\psi}+(p) \\
& -2\left\{\bar{\psi}-\left(p^{\prime}\right) \bar{\sigma} \cdot p \mathbf{p}^{\mu}[\bar{p} \cdot \mathbf{q}-(p)+m \bar{\psi}+(p)\}\right. \\
& \left.+\left[m \bar{\psi}-\left(p^{\prime}\right)-0 \cdot q \psi\left(p^{\prime}\right)\right] \partial \nu^{\nu} \cdot p^{\prime} \bar{w}+\left(p^{\prime}\right)\right\} \\
& +2\left\{\bar{v}^{\prime}-\left(p^{\prime}\right) \sigma \cdot\left(p x+p^{\prime} y\right) a^{\prime \prime}[z \cdot q \psi-(p)+m \bar{\psi}+(p)]\right. \\
& +\bar{\psi}-\left(p^{\prime}\right) \delta \cdot\left(p-p x-p^{\prime} y\right) \sigma^{\mu}\{y d \cdot q \psi(p)+m(x+y) \bar{\psi}+(p)\} \\
& +\left\{\left(m(x+v) \bar{\psi}-(p)-x \psi_{+}+(p) \sigma \cdot q\right) \sigma^{\prime \prime} \sigma \cdot\left(p^{\prime}-p x-p \cdot y\right) \bar{\psi}+(p)\right. \\
& \left.+[m \bar{\psi}-(p)-\psi+(p) \alpha \cdot q] \partial \omega^{\sigma} \cdot\left(p x+p^{\prime} y\right) \bar{\psi}+(p)\right\} \\
& +2 m\left\{2(1-x-y) m \bar{\psi}-\left(p^{\prime}\right) \sigma^{m} \psi_{-}(p)-(1-2 x) \psi_{+}(p) \sigma \cdot q \sigma \psi_{-}(p)\right\} \\
& +2 m \psi_{+}\left(p^{\prime}\right) 0^{\mu}\{2 m(1-x-y) \bar{\psi}+(p)+\bar{\psi} \cdot q(1-2 y) \psi-(p)\}
\end{aligned}
$$

Collecting terms as using Eqqn. (B.2) again, i.e. $\psi-(p)=(\sigma \cdot p / m) \bar{\psi}+(p)$ end $\psi+\left(p^{\prime}\right)=\bar{\psi}\left(p^{\prime}\right)(\delta \cdot p / m)$, we oblein (after doinz some algebra): 
Thus considering only the fret term and noting that

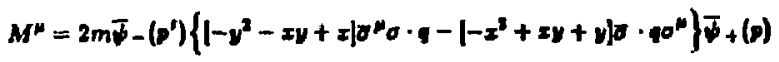

$$
\begin{aligned}
& \left.+2 m^{3} \bar{\psi}-\left(p^{\prime}\right)\left\{\sigma^{n} \mid-(1-x-y)^{2}+(1-2 x-2 y)\right\}\right\}-(p) \\
& +2 m^{2} \psi_{+}\left(p^{\prime}\right)\left\{o^{\mu}\left[-(1-x-y)^{2}+(1-2 x-2 y)\right\} \bar{\psi}+(p)\right. \\
& +2 m \psi_{+}\left(p^{\prime}\right)\left\{\left(-x y-y^{2}+x\right) \sigma^{n} z \cdot q+\left(x^{2}+x y-y\right) \sigma \cdot \rho^{n}\right\} \psi-(r)
\end{aligned}
$$

We now once again use the " bt" trick of Egg. (D.10). By switching what we call " $b$ " and " $c$ " wt, in entente, witch $x \rightarrow y$ in the integrand of (C.e) Doing this we obtain (D.39) but with $x \rightarrow y$. Averaging (D.39) with this new equation wo find that

$$
\begin{aligned}
& M^{\mu}=2 m \bar{\psi}-\left(p^{\prime}\right\}\left\{f\left|(x+\nu)-(x+y)^{2}\right|\left|\sigma \omega_{\sigma} \cdot-\sigma \cdot \sigma^{\mu}\right|\right\} \bar{\phi}+(p) \\
& +2 m^{2}\left[(1-x-y)-(1-x-y)^{2}-(x+y)\right] \\
& x\left(\bar{\psi}-\left(p^{\prime}\right) \psi-(p)+\psi+(p) \omega^{\psi}+(p)\right)
\end{aligned}
$$

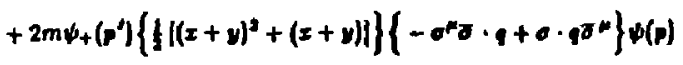

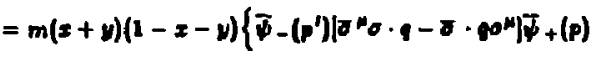

$$
\begin{aligned}
& \left.+\psi_{+}\left(p^{\prime}\right)\left|\sigma^{\mu} \sigma \cdot q-\sigma \cdot q^{\mu}\right| \psi-(p)\right\} \\
& -2 m^{2}(x+y)^{2}\left\{\bar{\psi}-\left(p^{\prime}\right) \sigma^{\mu} \psi-(p)+\psi_{+}\left(p^{\prime}\right) \sigma^{\mu} \bar{\psi}+(p)\right\}
\end{aligned}
$$

and the final term is proportional to the $\gamma^{\mu}$ term (ide. vertex renormalization).

105

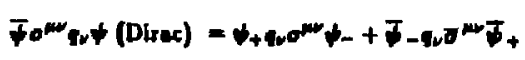

(from (A.8))

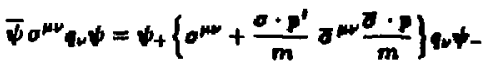

$$
\begin{aligned}
& =\frac{i}{2 m} v+\left\{m \mid a^{n}-\theta^{2}-a^{v}-m\right\}
\end{aligned}
$$

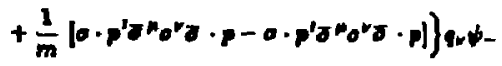

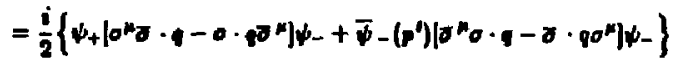

From (D.42) the frat term in (D.40) is

$$
=m(x+y)(1-x-y) \frac{1}{(i / 2)} \bar{\psi} \sigma^{\mu+\nu \psi}
$$

(using Dirac spinet notation for short). From (D.32)

$$
M^{N}=-\bar{v} \sigma^{\mu \nu} q \psi \frac{-i \varepsilon^{2}}{(i / 2)} \frac{i}{16 n^{2}} \int_{\omega^{2}}^{4} d \int_{0}^{2} d x \int_{0}^{1-2} d y \frac{m(x+y)(1-x-y)^{2}}{\left|-1(1-x-y)-m^{2}(x+y)^{2}\right|^{2}}
$$

Since

$$
\Gamma^{\mu}\left(p, p^{q}\right)=q^{\mu} F_{1}\left(q^{2}\right)+\frac{i}{2 m} \sigma^{\mu \nu} q_{\nu} F_{2}\left(q^{2}\right)
$$

with

$$
\Delta a_{0} \equiv F_{2}\left(a^{2} \rightarrow 0\right) \equiv \frac{q-2}{2}
$$

100 
we have

$$
\Delta a_{e}=\frac{2 m}{i} \cdot \frac{-i e^{2}}{i / 2} \cdot \frac{i}{i 6 \pi^{3}} \int_{m^{3}}^{\lambda^{3}} d t \int_{0}^{1} d x \int_{0}^{1} d x \frac{m z(1-2)^{3}}{\left|(1-x)+m^{2} x^{2}\right|}
$$

and 20

$$
\begin{aligned}
& \Delta a_{n}=-\frac{m^{2} e^{2}}{4 \pi^{2}} \int_{n}^{n} d x \int_{0}^{1} d x \int_{1}^{D} d z \frac{x(1-n)^{2}}{\left[(1-x)+m^{3} z^{2}\right]^{2}} \\
& =\left.\frac{m^{2} a}{\pi} \int_{0}^{1} d x \int_{1}^{k} d z \frac{(1-2) x}{(t-x)+m^{2} x^{2}}\right|_{\mu^{2}-0} ^{n^{3}-\infty} \\
& =-\frac{m^{2} a}{\pi} \int_{0}^{1} d x d z \frac{z(1-x)}{m^{2} z^{3}} \\
& =-\frac{0}{\pi} \int_{0}^{1} d x[\ln x-x+1] \\
& =\frac{a}{2 \pi} \text {. }
\end{aligned}
$$

\section{FIGURE CAPTIONS}

1. Naive contributions to $e_{L}+\tau \rightarrow \mathrm{CR}_{R}$.

Here $\Delta(p) \equiv i / p^{2}-m^{2}+i c$.

2. Additional Diagrams Contributing to $e z+q \rightarrow e R$.

Thus we oblain the correct result again using this more involved method. 

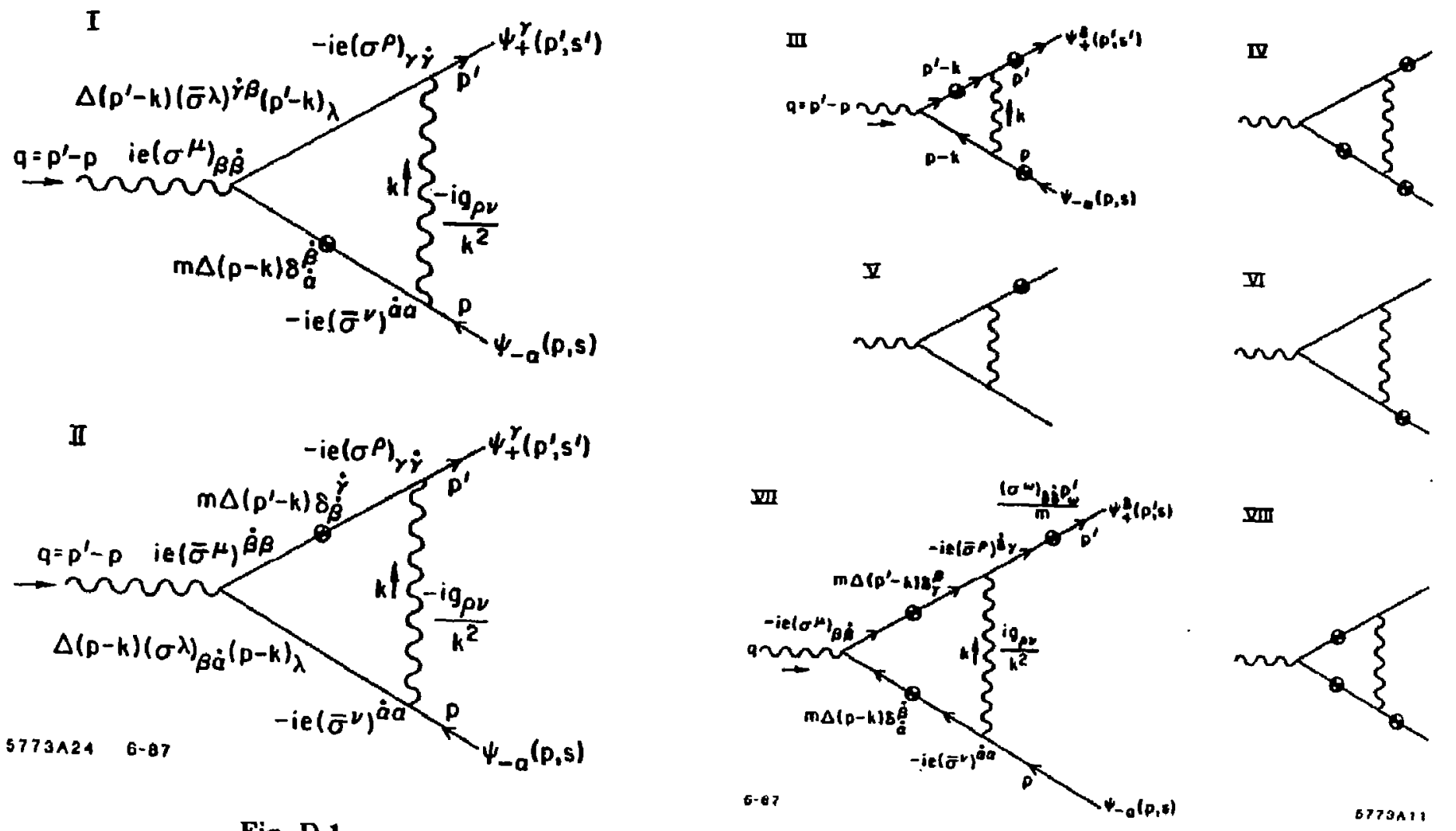

Fig. D.1

Fig. D.2

100 


\section{APPENDIX E}

Superaymmetric QED Contribution to Anomalous Moment

We wish to consider $\Delta a_{e}$, the additional contribution to the anomalous mag. netic moment of the electron, arising from a minimal supersymmetric extension or QED.

\section{E.I LAGRANGIAN FOR SUPERSYMAIETRIC QED (SQED)}

We ure the following notation ${ }^{11}$ for the superfields and their components:

\begin{tabular}{|c|c|c|}
\hline Superfield & Ordinary Matter & Superpartners \\
\hline$\checkmark$ & $\boldsymbol{T}$ & $\lambda$ \\
\hline $\mathbf{L}_{\mathbf{i}}$ & $l_{L_{t}}^{-}$ & $i_{L t}$ \\
\hline $\mathbf{f}_{i}$ & $\left(\ell_{i}^{+}\right)_{L}=\left(l_{B}^{-}\right)^{*}$ & $\left(i_{R}\right)^{\circ}$ \\
\hline
\end{tabular}

Note that when we consider a full electroweak theory that $\boldsymbol{L}_{i}$ will will be pro moled to a weak doublet which will include neutrinot and their euperaymmetrit counterparts. The seneration index " $i$ " will now be dropped a we proceed to specialize to a single generation.

The superfield strengths are

$$
\begin{aligned}
& w^{\alpha}=-\left\{\bar{D} \bar{D} D^{\circ} \hat{v}\right. \\
& \bar{W}_{\alpha}=-\left\{D D \bar{D}_{\delta} v\right.
\end{aligned}
$$

and

$$
\begin{aligned}
\mathcal{L}_{S Q E D}= & \int d^{2} O d^{2} \bar{\delta}\left\{\frac{1}{\delta}\left(W W \delta\left(\bar{\sigma}^{2}\right)+W W \delta\left(0^{2}\right)\right)\right. \\
& +L e^{-\Delta V} L+R e^{\varepsilon V} R+m_{e}\left(\left(R L \delta\left(\sigma^{2}\right)+L R \delta\left(0^{2}\right)\right)\right\}
\end{aligned}
$$

where $\delta(\theta)=0$ and $\delta(\overline{0})=\delta$ dya to the rules of Grassman integration (see Appendix I fer deteiss of this casstrurtion). Expanding this out in component fields and eliminating the auxiliany fields $F_{ \pm}$and $D$ vie their equations of motion yields (for electrons only)

$$
\begin{aligned}
& \mathcal{L}_{S Q E D}=-j F_{\mu \nu} F^{\mu \nu}-i \lambda \sigma^{\mu} \partial_{\mu} \bar{\Lambda}+\tilde{e}_{R} \square \tilde{e}_{B^{*}}+\tilde{\tau}_{L}{ }^{*} \square \tilde{e}_{L} \\
& +i\left(\partial_{\mu} \bar{\psi}_{+} \sigma^{\mu} \psi_{+}+\partial_{\mu} \bar{\psi}_{-} \sigma^{\mu} \psi_{+}\right) \\
& +\frac{1}{2} e \lambda_{\mu}\left[\bar{\psi}_{+} \sigma^{\mu} \psi_{+}-\bar{\psi}_{-}-\sigma^{\mu} \psi_{-}\right. \\
& \left.+i\left(\tilde{e}_{R} \partial^{\mu} \tilde{e}_{R} \cdot-\partial^{\mu} \tilde{e}_{R^{2}} \tilde{e}_{R^{\prime}}-\tilde{e}_{L^{\prime}} \partial^{\mu} \tilde{e}_{L}+\partial^{\mu} \tilde{e}_{L} \cdot \tilde{e}_{L}\right)\right] \\
& -\frac{i e}{\sqrt{2}}\left[\tilde{e}_{R} \cdot \bar{\psi}+\bar{\lambda}-\tilde{e}_{R} \psi_{+} \lambda-\bar{\varepsilon}_{L} \bar{\psi}_{-} \bar{\lambda}+\bar{\varepsilon}_{L} \cdot \psi_{-} \lambda\right]
\end{aligned}
$$

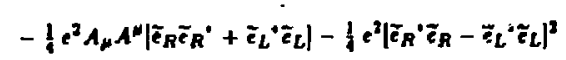

$$
\begin{aligned}
& -m\left|\psi_{+} \psi_{-}-\bar{\psi}+\bar{\psi}-\right|-m_{e_{n}^{2}}^{z_{R}} \tilde{\tau}_{R} \tilde{c}_{R} \\
& -m_{\tilde{k}_{L}}^{2} \tilde{\varepsilon}_{L} \tilde{\varepsilon}_{L}-m_{\lambda} T \lambda
\end{aligned}
$$

In unbroken SQED $m_{\tilde{e}_{2}}=m_{\bar{e}_{n}}=m_{e}$ and $m_{\lambda} \equiv m_{j}=0$. Here explicit breaking terms have been added to give $\tilde{E}_{L}, \tilde{e}_{R}$ and $\tilde{\gamma}$ arbitrary masses. Presumably such 
terms would arice in a realistic model as effective residue of come untpecifed higher-energy theary. Note that $\tilde{c}_{L}$ and $\bar{\tau}_{R}$ we independent field.

\section{E.2 Feynman Rutes}

i) There are four types of verticen in this theory. Since QED in left-right blind

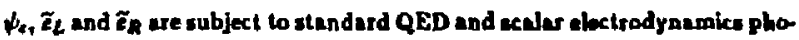
ponic coupling vertices. There are aleo Yukawe vertices $c_{L} \tilde{c}_{L} \tilde{\gamma}$ and $\varepsilon_{\pi} \tilde{\tilde{F}_{n}} \tilde{\gamma}$ of coupling strength $\sqrt{2} \mathrm{c}$.

ii) $\psi_{e}$ and $\tilde{\tau}$ have atendard (Wey) formionic propagatore $(\tilde{f}$ is $:$ Majorant particle) and $\tilde{c}_{L_{2}}$ have standurd acalas propagntors.

iii) In conxidering $\Delta a_{n}$, there are no diegrame with a chisality change in the photino propagator since that would require $\tilde{\tilde{C}}_{\mathbf{L}}-\tilde{\boldsymbol{\tau}}_{\boldsymbol{R}}$ mixing in $\boldsymbol{L}$. Thus wo find, when looking for $\epsilon_{\downarrow}+\gamma \rightarrow e_{R}$, that only the two disgrame of $F_{3}$. 1 contribute.

\section{E.3 COMPUTATION OF DJAGRAM 1}

$$
\begin{aligned}
& \mu^{\mu} \equiv-i e \psi_{+}^{p}\left(p^{\prime}, s^{\prime}\right) \Gamma_{,}^{\mu}\left(p^{\prime}, s\right) \beta^{o} \psi_{-o}(p, a) \\
& =(-i e)^{3} 2\left(i^{3}\right) \int \frac{d^{4} k}{(2 \pi)^{4}}
\end{aligned}
$$

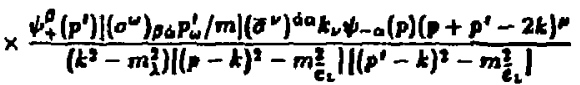

We shall work on shell so that $p^{2}=p^{2 z}=m_{E}^{2} \equiv m^{2}$.
Define

$$
\begin{aligned}
& m_{l} \equiv m^{2}-m_{l_{l}}^{2}+m_{l}^{l} \\
& m_{R}^{2} \equiv m^{2}-m_{c_{k}}^{2}+m_{l}^{2}
\end{aligned}
$$

frotn Eqn. (B.6)

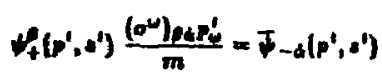

then (E.3) beeome

$$
\begin{aligned}
& \psi_{+} \Gamma_{F} \psi_{-}=2 i e^{2} \int \frac{N^{4}}{(2+)^{2}} \frac{N^{\mu}}{D}
\end{aligned}
$$

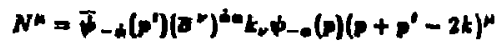

$$
\begin{aligned}
& D=\left(k^{2}-m_{\lambda}\right)^{2}\left(k^{2}-2 p \cdot k+m^{2}-m_{C_{L}}^{2}\right)\left(k^{2}-2 p^{\prime} \cdot k+m^{2}-m_{C_{L}}^{2}\right)
\end{aligned}
$$

We now use Eqn. (D.11) to And

$$
\frac{1}{D}=2 \int_{0}^{1} d x \int_{0}^{1} d y\left(k^{2}-2 k \cdot\left(p x+p^{\prime} y\right\}+\bar{m}\left\{\langle x+y)-m_{\lambda}^{2}\right)^{-3}\right.
$$

We next use Eqgn. (D.12) or (H.6) to find

$$
\psi_{+} \Gamma_{j}^{\mu} \psi_{-}=2 i e^{2} \cdot 2 \int_{0}^{1} d x \int_{0}^{1-x} d y
$$

$$
\times \frac{\left.i \psi_{-\alpha}\left(z^{\prime}\right)^{\alpha q} \mid p x+p^{\prime} y\right)_{\nu} \psi_{-\alpha}\left(p+p^{r}-2\left(p x+p^{\prime} y\right)\right)^{\mu}}{32 \pi^{2}\left(\bar{m}_{L}^{2}(x+y)-m_{\lambda}^{2}-\left|p x+p^{\prime} y\right|^{2}\right)}
$$

where we have ignosed the $k_{\nu} k_{\mu}$ letm which contributes solely to the helicitypreservine piece of the amplitude (i.c. $\left.\sigma^{\mu}\right)$. Since $\left(p x+p^{\prime} y\right)^{2}=m^{2}(x+y)^{2}-q^{2} x y$ 
as before

$$
\begin{aligned}
\psi_{+} \Gamma_{I}^{\mu} \psi_{-}= & -\frac{e^{2}}{8 \pi^{2}} \int_{0}^{1} d x \int_{0}^{1-x} d y \\
& \times \frac{\bar{\psi}-d\left(\delta^{2}\right)^{d a}\left|p x+p^{\prime} y\right|_{\nu} \psi_{-a}\left|p(1-2 x)+p^{\prime}(1-2 y)\right|^{\mu}}{m_{L}^{2}(x+y)-m_{\lambda}^{?}(x+y)^{2}-s^{2} x y} .
\end{aligned}
$$

We now perform the " $b+c^{\prime \prime}$ averaging trick as in (D.19) The result being that $x \ldots y$ in the integsand of (E.8), and therefore (E.O), and then we weraze the two expressions. Before we do this it 5 prodent to apply the Dirac equatione of (B.2).

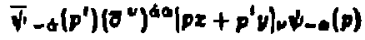

$$
\begin{aligned}
& =\bar{\psi}_{-\alpha}\left\{m x \bar{\psi} \psi+m y \bar{\psi}_{+}^{\delta}+\left(\sigma^{\nu}\right)^{\delta \alpha_{q}} \psi_{\psi-\alpha y}\right\}
\end{aligned}
$$

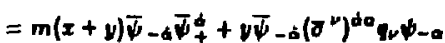

when we now perform the $x \rightarrow y$ overaze we find

$$
\begin{aligned}
& \psi_{+} \Gamma_{1}^{\mu} \psi_{-}=-\frac{e^{2}}{8 \pi^{2}} \int_{0}^{1} d x \int_{0}^{1-x} d y \frac{M \mu}{-\overline{m^{2}(x+y)^{2}+m_{L}^{2}(x+y)-m_{\lambda}^{2}-q^{2} x y}} \\
& M^{\mu}=m(x+v)(1-x-y)\left(p+\phi^{\prime}\right)^{\mu} \overline{\nu^{\prime}-\phi \bar{\psi}} \\
& +\left[y\left(p(1-2 x)+p^{\prime}(1-2 y)\right\}\right. \\
& \left.+x\left\{p(1-2 y)+p^{\prime}(1-2 x)\right\}\right] \bar{y}_{-d}\left(z^{2}\right)^{d o} \phi_{p} \psi-0
\end{aligned}
$$

We are intereated in the helicity-changing part of this (the $\bar{\psi}-a \bar{\psi}+$ lerm) as $\varphi^{2} \rightarrow 0$ (on shell). This is

$$
\psi_{+} \Gamma_{i L R}^{\mu}\left(q^{2} \rightarrow 0\right) \psi_{-}=\frac{c^{2}}{8 \pi^{2}} \frac{\left(p-p^{\prime}\right)^{\mu}}{m} \bar{\psi}_{-d} \bar{\psi}_{+}^{d} J_{L}
$$

where

$$
\begin{aligned}
J_{L} & \equiv \int_{0}^{1} d x \int_{0}^{1-2} d y \frac{(x+y)(1-x-y)}{-(x+y)^{2}+\frac{m^{2}}{m^{2}}(x+y)-\frac{m^{2}}{m^{2}}} \\
& =\int_{0}^{1} d x \int_{z}^{1} d z \frac{x(1-z)}{-z^{2}+\frac{m_{l}^{2}}{m^{2}} x-\frac{m^{2}}{m^{2}}}
\end{aligned}
$$

where we have let $z=x+y$.

Now in (E.10) we applied the Dirac operator to the right. We could equally well have had II operator to the lefl. Using Eqn. (B.z) again we would have obteined

$$
\begin{aligned}
& \bar{\psi}_{-d}\left(p^{\prime}\right)\left(z^{\nu}\right)^{d \sigma}\left|p s+p^{t} v\right|_{\nu \psi_{-\alpha}}(r) \\
& =\left\{\left|m \psi_{+}^{\circ}-\left(\sigma^{\nu}\right)^{\mathrm{d} \sigma} q_{\nu} \bar{\psi}_{-\infty}\right| x+m \psi_{+}^{0} y\right\} \psi_{-\infty} \\
& =m(x+y) \psi_{+}^{q} \psi_{-\bullet}- \pm \bar{\psi}-d\left(\partial^{v}\right)^{\alpha \alpha_{\nu}} \psi_{-a}
\end{aligned}
$$

(We use $+m$ since we are considering electrona and not positrona.) Note that in (E.10) we have reloted the $\mathrm{C}_{L} \rightarrow \mathrm{C}_{R}$ term to the corresponding $\mathrm{e}_{A} \rightarrow \mathrm{C}_{L}$ Lerma wheress $(E .14)$ expresses $e_{L} \rightarrow e_{R}$ in terms of $e_{L} \rightarrow e_{R}$. Usine $(E . .1)$ instead of (E.10) would change (E.12) to

$$
\psi_{+} \Gamma_{I L R}^{\mu}\left(q^{2}+0\right) \psi_{-}=\frac{e^{2}}{B \pi^{2}} \frac{\left(p+p^{\prime}\right)^{\mu}}{m} \psi_{+}^{0} \psi_{-a} J_{L} .
$$

Averaging (E.12) and (E.15) and using $\alpha=e^{2} / 4 \pi$

$$
\psi_{+} \Gamma_{i L n}^{\psi} \psi_{-}=\frac{a}{d x} \frac{\left(p+p^{\prime}\right)^{\mu}}{m}\left(\psi_{+}^{\circ} \psi_{-\infty}+\bar{\psi}_{-\phi} \bar{\psi}_{+}^{a} \mid I_{L} .\right.
$$


From Appendix H or Eqn. (D.23) we employ the Gordon decompoxition producine

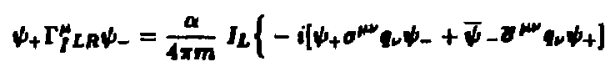

$$
\left.+2 m\left|\bar{\phi}-\sigma * \bar{\phi}++\phi_{+}+\phi \psi\right| 1\right\}
$$

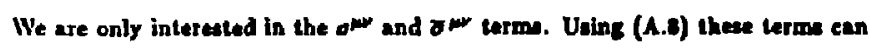
be combined to yield the Dirac apinor formulation:

$$
\bar{\psi} \Gamma_{I L R}^{\mu} \psi=\frac{-i \alpha}{1 \pi m} I_{L} \bar{\psi}_{\sigma}^{\mu \nu} q \psi
$$

From (C.15) and (C.16)

$$
\Delta a^{(L)}=\frac{\alpha}{2 \pi} J_{L}
$$

which is the $\tilde{\varepsilon}_{L}$ contribution to $\boldsymbol{a g}_{g}$.

\section{E.4 Computation of Diagram I AND TOtal Contninuton}

We ingeminate the steps of each in Section E.3 beginning with

$$
-i e \psi_{+} \Gamma_{I}^{\mu}\left(p, p^{\prime}\right) \psi_{-}=(-i e)^{2} 2\left(i^{3}\right) \int \frac{d^{*} k}{(2 \pi)^{4}}
$$

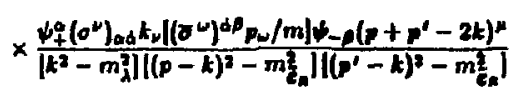

and conclude with

$$
\Delta a_{f}^{(R)}=\frac{\alpha}{2 \pi} J_{R}
$$

where

$$
I_{R}=\int_{0}^{1} d x \int_{z}^{1} d x \frac{z(1-x)}{-x^{2}+\frac{m^{2}}{m^{2}} x-\frac{m^{2}}{m^{2}}}
$$

and $\bar{m}_{R}$ is given by (E.6). The total contribution to $\Delta a_{2}$, the incremental super- symmetric contribution to the anomalous moment of the electron a, in

$$
\begin{aligned}
\Delta e_{a} & =\Delta d_{t}^{(L)}+\Delta e^{(R)} \\
& =\frac{a}{2 \pi}\left(J_{L}+J_{R}\right) \\
I_{L, R} & =\int_{0}^{1} d x \int_{x}^{1} d x \frac{x(1-x)}{-x^{2}+\frac{m^{2}+m_{\lambda}^{2}-m_{L_{L}}^{2}}{m^{2}}-\frac{m_{l}^{2}}{m^{2}}}
\end{aligned}
$$

E.6 Specific Cases

a) $m_{1}^{2}, m_{e}^{2}<m^{2}$. Thiv cuse is excluded experimentally. In this litait

$$
\begin{aligned}
& J_{L, E}=-\frac{1}{2} \\
& \Delta E_{E}=-\frac{a}{2 \pi} .
\end{aligned}
$$

This is of the anme matgitude as the QED contribution but of the opposite sign.

b) $m_{\lambda}^{k} \approx m_{\varepsilon}^{2}>m$. Rather unlikely in most models but not excluded.

$$
\begin{aligned}
& I_{L, R}=\int_{0}^{1} d x \int_{a}^{1} d x \frac{m^{2}}{m_{l}^{2}} x(x-1)=-\frac{1}{12} \frac{m^{2}}{m_{l}^{2}} \\
& \Delta \sigma_{l}=-\frac{a}{24 \pi} \frac{m^{2}}{m_{\lambda}^{2}}
\end{aligned}
$$

c) $m_{e}^{2} \rightarrow m_{d}^{2}>m^{2}$. The standardly presumed scenario.

$$
\begin{aligned}
& I_{L, R}=\int_{0}^{1} d x \int_{a}^{t} d x \frac{m^{2}}{\bar{m}_{L_{1, n}}^{2}}(1-x)=-\frac{1}{6} \frac{m^{2}}{m_{L_{L, A}}^{2}} \\
& \Delta a_{k}=-\frac{\alpha}{12}\left\{\frac{m^{2}}{m_{e_{L}}^{2}}+\frac{m^{2}}{m_{e_{n}}^{2}}\right\}=\Delta a_{c}^{(m+=0)}
\end{aligned}
$$


This is precisely the 1974 result of Fayet."

d) $m_{i_{t}}=m_{i_{n}}=m ; m_{\lambda}=0$. Case of exuct supersymmetry

$$
\begin{aligned}
I_{L, R} & =\int_{0}^{1} d x \int_{1}^{1} d x \frac{z(1-f)}{z^{2}}=-\frac{1}{2} \\
\Delta a_{E}^{(S U S Y)} & =-\frac{a}{2 \pi}=-a_{l}^{(O E D)} .
\end{aligned}
$$

In the exact supersymmetric limit we expect that there will be no nnomlous magnetic moment to any order. Therefore we should have anticipated thet $\Delta a f^{(s U S Y)}$ would precisely cancel $f^{(G E D)}$.

e) $m_{2}=0$

$$
\begin{aligned}
& I_{L, R}=\int_{0}^{1} d x \int_{0}^{1} d z \frac{1-z}{-(1-z)} \quad \cap \pm \frac{m_{E_{L, k}}^{2}}{m^{2}} \\
& =\rho(1-\rho)|\ln \rho-\ln (\rho-1)|-\rho+\frac{1}{2}
\end{aligned}
$$

f) $m_{\lambda}, m_{\dot{e}}>m$. This is the most interestins case.

Definine

$$
\begin{aligned}
& R_{L, A}=\frac{m_{\lambda}^{2}}{\bar{m} l_{, R}}=\frac{m_{\lambda}^{3}}{m_{\lambda}^{2}-m_{t_{L, A}}^{2}+m^{2}} \\
& I_{L, R}=\frac{m^{2}}{m_{L, R}^{2}} \int_{0}^{l} d x \int_{z}^{l} d z \frac{z(1-x)}{z-\bar{K}_{L, R}}
\end{aligned}
$$

noting that

$$
\int_{0}^{1} d x \int_{0}^{1} d z=\int_{0}^{1} d z \int_{0}^{1} d z
$$

where $\tilde{z}=2 x-2$

$$
\begin{gathered}
I_{L, R}=\frac{m^{2}}{\bar{m}^{2}} \int_{0}^{1} d z \frac{z^{2}-z^{2}}{z-R} \\
I=\frac{m^{2}}{\bar{m}^{2}}\left[\frac{1}{6}+\frac{1}{2} R-R^{2}+R^{2}(1-R) \ln \left(\frac{R-1}{R}\right)\right] .
\end{gathered}
$$

Now we note that

$$
\frac{m^{2}}{\bar{m}^{2}}=-\frac{m^{2}}{m_{E}^{2}}\left(1-\left[1+\frac{m^{2}}{m_{2}^{2}}\right] R\right) \approx-\frac{m^{2}}{m_{E}^{2}}(1-R)
$$

if $m<m_{\lambda}$ so

$$
I=-\frac{m^{2}}{m_{e}^{2}}|1-R| h(R)
$$

where

$$
h(R)=\frac{1}{6}+\frac{1}{2} R-R^{2}+R^{2}(1-R) \ln \left(\frac{R-1}{R}\right) .
$$

The $" 1 / 6^{*}$ term is what we lound in part $(c)$ when $m, \rightarrow$. This is herdly shocking tince as $m_{\lambda} \rightarrow 0$ we heve $R \rightarrow 0$ and $(1-R) h(R) \rightarrow 1 / 6$. Factoring this term out we can write

$$
\begin{aligned}
& L_{L, R}=-\frac{m^{2}}{6 m_{L, n}^{2}}\left[1+J\left(R_{L, h}\right)\right] \\
& f(R)=2 R-9 R^{2}+6 R^{3}+6 K^{2}(1-R)^{2} \ln \left(\frac{R-1}{R}\right)
\end{aligned}
$$

and from (E.23) and (E.24)

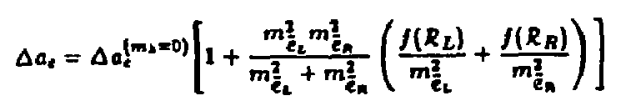


where

FIGURE CAPTIONS

$$
R_{L, x} \pm \frac{m_{L}^{2}}{m_{\lambda}^{2}-m_{L_{L}}^{2}}
$$

since $m_{\lambda}>m$.

Notes:

(i) when $m_{\varepsilon}>m_{\lambda} R \rightarrow 0$ and $f(R) \rightarrow 0$. Then $\Delta t_{4} \rightarrow \Delta d^{\left(m_{1}=0\right)}$ to bowel order.

(ii) The frat order correction in the above 1 to $O\left(\mathrm{~m}_{\mathrm{M}} / \mathrm{m}\right)$, comes from noting that $f(R)=2 R$ a $R \rightarrow 0$ where $R \div-m ! / m\}$. Thus $(E .31)$ become

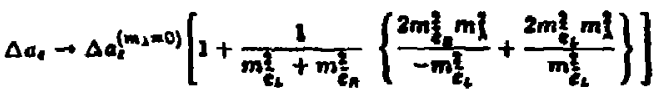

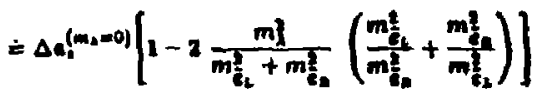

(iii) When $\left.m_{\lambda}>m_{i}>m \quad R \rightarrow 1+m\right\} / m_{\lambda}^{2}$ and $f(R) \rightarrow-1$. Using (E.20) we see that $I \rightarrow[1-R](-1 / 3)=-1 \frac{m^{2}}{m g}$ just as in case (s) which follows. g) $m_{k}>m_{e}>m$. This case is also unlikely in most models but is not excluded.

$$
\begin{aligned}
& I_{L, A}=\int_{0}^{1} d t \int_{0}^{\pi} d \tilde{x} \frac{m^{2} x(1-z)}{-m^{2} \alpha^{2}+\left(m^{2}-m^{2}+m^{2}\right)-m^{2}} \\
& =\frac{m^{2}}{m_{\lambda}^{2}} \int_{0}^{1} d t \int_{0}^{E} d x \frac{2(1-2)}{z-1}=-\frac{1}{3} \frac{m^{2}}{m_{2}^{2}} \\
& \Delta a_{2}=-\frac{a}{3} \frac{m^{2}}{m_{l}^{2}} .
\end{aligned}
$$

1. Contribution $10 \Delta \varepsilon_{4}$ when $\tilde{\tau}_{L}$ and $\tilde{\tau}_{R}$ are unmixed. Here $\Delta(p)=i / p^{2}-m^{2}+i e$.

121

122 

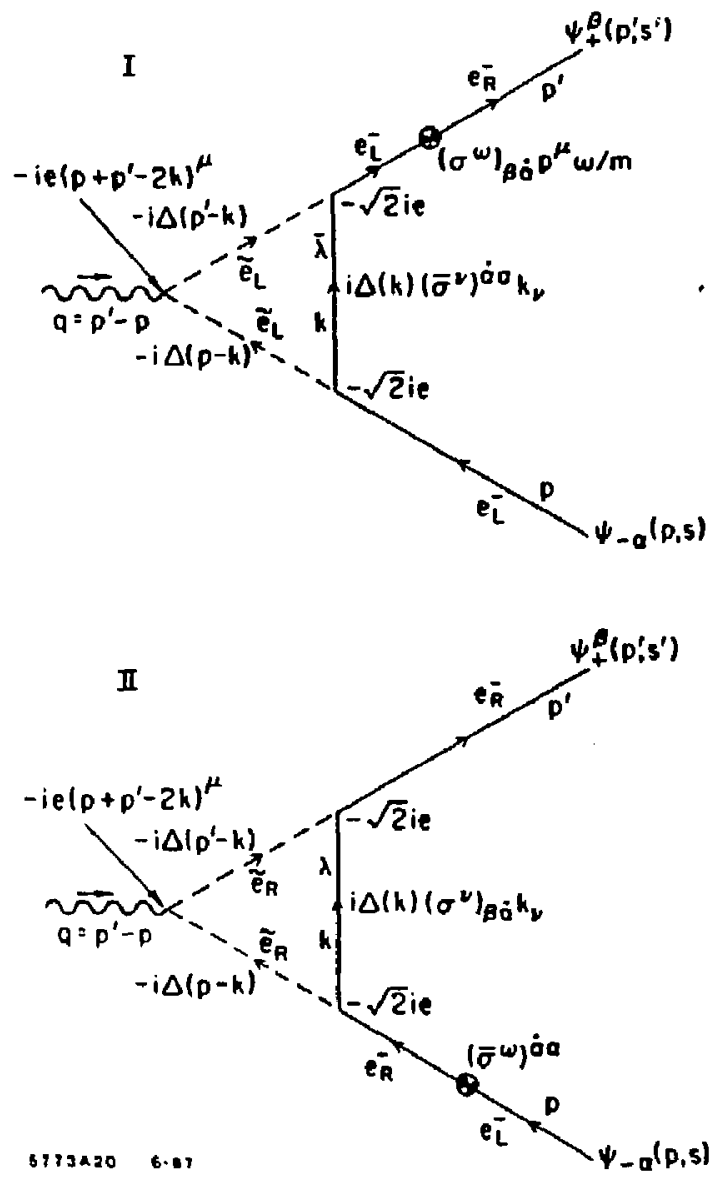

Fig. E.1

\section{APPENDIX F}

Contribution to Anomatous Moment from Smuon Mixins

\section{F.l Lagramgian}

We permit the twosmuons $\bar{\mu}_{L}$ and $\bar{\mu}_{R}$ to mix resulting in the mass eigenstates

$$
\begin{aligned}
& \bar{\mu}_{1}=\cos \phi \tilde{\mu}_{L}+\sin \phi \tilde{\mu}_{n} \\
& \bar{\mu}_{2}=-\sin \phi \tilde{\mu}_{L}+\cos \phi \tilde{\mu}_{n}
\end{aligned}
$$

or equivalently

$$
\begin{aligned}
& \tilde{\mu}_{L}=\cos \phi \tilde{\mu}_{1}-\sin \phi \tilde{\mu}_{2} \\
& \tilde{\mu}_{R}=\sin \phi \tilde{\mu}_{1}+\cos \phi \tilde{\mu}_{2} .
\end{aligned}
$$

We will confine ourselves to the muon here while recosnizing that the same analyzis toes through for the electron and tau. It is the muon which provides the mont stringent bounds from experiment. We assume that $\lambda=(\tilde{7})$ is purely Majorana, olthaugh it may be marsive (R-parity is always to be considered an inviolable symmetry).

The terms in the Lazrangian of Appendix E (E.4) are altered thusly

$$
\begin{aligned}
\text { Crulaw } \rightarrow & -\frac{i e}{\sqrt{2}}\left[\sin \phi \bar{\mu}_{1} \cdot \bar{\psi}+\bar{\lambda}+\cos \phi \tilde{\mu}_{2} \cdot \bar{\psi}+\bar{\lambda}\right. \\
& -\sin \phi \bar{\mu}_{1} \psi_{+} \lambda-\cos \phi \tilde{\mu}_{2} \psi_{+} \lambda \\
& -\cos \phi \bar{\mu}_{1} \bar{\psi}_{-} \bar{\lambda}+\sin \phi \tilde{\mu}_{2} \bar{\psi}-\bar{\lambda} \\
& \left.+\cos \phi \tilde{\mu}_{1} \cdot \psi_{-} \lambda-\sin \phi \tilde{\mu}_{2}^{*} \psi_{-} \lambda\right] .
\end{aligned}
$$


Where $\&$ represents the muon feld.

$$
\mathfrak{L}_{\text {MASs }} \rightarrow-m \tilde{H}_{1} \tilde{\mu}_{1} \cdot \tilde{\mu}_{1}-\boldsymbol{m} \boldsymbol{l}_{1} \tilde{\mu}_{2} \cdot \tilde{\mu}_{2}
$$

$\mathcal{L}_{\text {Ecaller-Photoz }} \rightarrow \frac{i}{2} \in A_{\nu}\left[\sin ^{2} \phi\left(\tilde{\mu}_{1} \partial^{\nu} \tilde{\mu}_{1} \cdot-\tilde{\mu}_{1} \cdot \partial^{\nu} \tilde{\mu}_{1}\right)+\cos ^{2} \phi\left(\tilde{\mu}_{2} \partial^{\nu} \tilde{\mu}_{2} \cdot-\tilde{\mu}_{2} \cdot \partial^{\nu} \tilde{\mu}_{2}\right)\right.$

$$
+\operatorname{tin} \phi \cos \phi\left(\tilde{\mu}_{1} \partial^{\nu} \tilde{\mu}_{2} \cdot+\tilde{\mu}_{2} \partial^{\mu} \tilde{\mu}_{1} \cdot-\tilde{\mu}_{2} \cdot \partial^{\mu} \tilde{\mu}_{1}-\tilde{\mu}_{1} \cdot \partial^{\nu} \tilde{\mu}_{2}\right)
$$

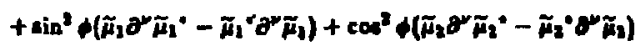$$
\left.-\sin \phi \cos \phi\left(\tilde{\mu}_{1} \partial^{\nu} \tilde{\mu}_{2}^{*}+\tilde{\mu}_{2} \partial^{\nu} \tilde{\mu}_{2}^{*}-\tilde{\mu}_{2} \cdot \partial^{\nu} \tilde{\mu}_{1}-\tilde{\mu}_{1}^{*} \partial^{2} \tilde{\mu}_{2}\right)\right]
$$

$$
=\frac{i}{2} e A_{2}\left[\tilde{\mu}_{3} \partial^{\nu} \tilde{\mu}_{1} \cdot-\tilde{\mu}_{1} \cdot \partial^{\nu} \tilde{\mu}_{1}+\tilde{\mu}_{2} \partial^{\nu} \tilde{\mu}_{2} \cdot-\tilde{\mu}_{2} \cdot \partial^{\nu} \tilde{\mu}_{2}\right]
$$

\section{F.2 feYnman RULE and Diagrams I-IV}

The Feynsos Rules are similar to thoee of Section E.2 except for the changes illustrated in Fig. F.1. Note that there is no $\bar{\mu}_{1} \bar{\mu}_{2} \gamma$ vertax (Fig. F.11) but the helicity-flipped photino propagator is now utilized.

In Fig. F.2 the loweat level contributions to $\mu_{L}+\gamma \rightarrow \mu_{R}$ are illuatrated. Diagrams I-W are reminiscent of the contributions of Fig. E.1. Indeed, if $m_{\tilde{j}_{2}}=m_{j_{3}}$, it is evident that these would aum to the unmixed result (we could rotate the mixing away all tozether and make diagrame V.VIII vanish recovering the provious configuration). The new features appen in diagrems $V$ to Vull. Here the helicity tip takes place in the photino line instead of the muon.
We may evaluate the int four diagrame by direct compesison with thoes of the previous appendix. Comparing 1 and 1 of Piz. F.2 with 1 of Pis. E.1

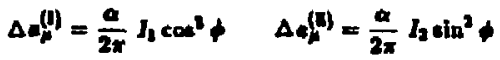

$$
\begin{aligned}
& I_{1,2}=\int_{0}^{1} d x \int_{0}^{1} d x \frac{z(1-x)}{-x^{2}+\frac{m_{l}^{2}}{m_{1}^{2}} x-\frac{m_{l}^{2}}{m_{0}^{2}}} \\
& \boldsymbol{m}_{1,2}^{2}=m_{\lambda}-m_{w_{1,0}}^{2}+m_{\mu}^{2}
\end{aligned}
$$

and, as before, we nole that we can wrile thb usine

$$
\int_{0}^{1} d x \int_{0}^{1} d x=\int_{0}^{1} d x \int_{0}^{1} d \pi x \int_{0}^{1} d x
$$

since there is no $x$ dependence and $\tilde{x}=2 x-x$. Similarly dhareme of and $\mathrm{W}$ give

$$
\Delta A(N)=\frac{\alpha}{2 \pi} L_{1} \sin ^{2} \phi \quad \Delta \alpha^{(N)}=\frac{\alpha}{2 \pi} I_{b} \cos ^{2} \phi
$$

and so the aum of the lirst four diegrume gives

$$
\Delta e^{(p-N)}=-\frac{\alpha}{2 \pi}\left(h_{1}+J_{1}\right)
$$

This in itself would place no aterner limlis than did (E.23):

$$
\Delta \Delta_{e}^{(-n+m i z e d)}=\frac{a}{2 \pi}\left(I_{L}+I_{R}\right)
$$

In the limit $m_{\tilde{\mu}_{1, a}}>m_{\lambda}>m_{\mu}$ (F.E) becomes

$$
\Delta a_{\mu}=-\alpha^{2} \pi\left\{\frac{m_{\mu}^{2}}{m_{\mu_{1}}^{2}}+\frac{m_{\mu}^{2}}{m_{k, 2}^{2}}\right\}
$$


which is essentially the same as the unmixed version

$$
\Delta a_{\mu}=-\frac{a}{2 n}\left\{\frac{m_{p}^{2}}{m_{\mu_{L}}^{2}}+\frac{m_{m}^{2}}{m_{m}^{2}}\right\}
$$

presenled in (E.24).

\section{F.3 Evaluation of Diagramis V.VIII}

Dimis!am V:

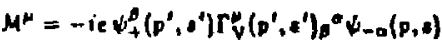

$$
\begin{aligned}
& =(-i c)^{3}(\sqrt{2})^{2}\left(i^{3}\right) \int \frac{d k}{(2 \pi)^{4}}
\end{aligned}
$$

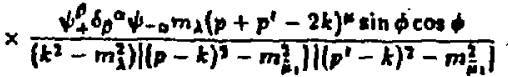

Following the steps detailed in Section E.3 we find $\left(m_{l}^{2}=m_{\lambda}^{3}-m_{p_{1}}^{2}+m_{p}^{2}\right)$

$$
\psi_{+} \Gamma_{v}^{\mu} \psi_{-}=\frac{i c^{2}}{8 \pi^{2}} m_{\lambda} \int_{0}^{l} d x \int_{0}^{1-2} d y \frac{i \psi_{q}^{q} \psi_{-o}\left(p+p^{\prime}-2\left(p x-p^{\prime} y\right)\right)^{\mu} \sin \phi \cos \phi}{m_{i}^{2}(x+y)-m_{\lambda}^{2}-m_{k}^{2}(x+v)^{2}-q^{2} x y} .
$$

Peyforming the "bacn trick w when we wes: from (E.6) to (E.11)

$$
\begin{aligned}
\psi_{+} r_{v}^{\mu} \psi_{-}= & \frac{a m_{\lambda}}{2 \pi} \sin \phi \cos \phi \psi_{+} \psi_{-} \int_{0}^{1} d x \int_{0}^{1-z} d y \\
& x \frac{\left(p+p^{\prime}\right] m^{n}(1-x-y)}{-\left|m_{j}^{2}(x+y)^{2}+m_{j}^{2}(x+;)-m_{1}^{2}-q^{2} x y\right|} .
\end{aligned}
$$

From Appendix H or Eqn. (D.23), or eimply following the step from (E.17) to
(E.19), we use the Gordon decomposition to find on shell $\left(q^{2}=0\right)$

$$
\Delta a l v)=\frac{a}{2 \pi} \frac{m_{\mu} m_{\lambda}}{m_{\mu}^{2}} I_{1}
$$

with

$$
\begin{aligned}
& J_{1}=\int_{0}^{1} d x \int_{0}^{1-2} d y \frac{1-x-y}{-(x+y)^{2}+\frac{m_{j}^{2}}{m_{0}^{2}}(x+y)-\frac{m_{l}^{2}}{m^{2}}} \\
& =\frac{m_{y}^{2}}{m_{1}^{2}} \int_{0}^{1} d x \int_{0}^{E} d \tilde{z} \frac{1-z}{-\frac{m_{0}^{2}}{m_{1}^{2}} z^{2}+z-R_{1}} \\
& z=x+y \quad \tilde{x}=x-y \quad R_{1}=m_{\lambda}^{2} / m_{1}^{2}
\end{aligned}
$$

where we have used notation similas to that of (E.26).

Diagram $V$ only provides the $\psi_{+} \sigma^{\mu \nu} q_{u} \psi_{-}$part of this term. The $\bar{\psi}-\gamma^{\mu \nu} q_{\nu} \psi_{+}$ piece comes from Diagram VII.

In the most interesting lirnit, when $m_{\lambda}, m_{k_{1}}>m_{\mu}$ (and cssuming $m_{1}>m_{*}$ ) we may expand this in powers of $m_{p=}^{2} / \bar{m}_{1}^{2}$. The lending o:der term is easy to find:

$$
\begin{aligned}
J_{1} & =\frac{m_{l}^{2}}{m_{i}^{2}} \int_{0}^{1} d z \frac{z(1-z)}{z-R_{1}}=\frac{m_{l}^{2}}{m !} \int_{-R_{1}}^{1-R_{1}} d s \frac{-s^{2}+\left(1-2 R_{1}\right) s+R_{1}\left(1-R_{1}\right)}{s} \\
& =\frac{m_{i}^{2}}{m_{1}^{2}}\left\{l\left(1-2 R_{1}\right)+R_{1}\left(1-R_{1}\right) \ln \left(\frac{R_{1}-1}{R_{1}}\right)\right\}
\end{aligned}
$$

which may be written, inciuding the next order term in $m_{\mu}^{2}$.

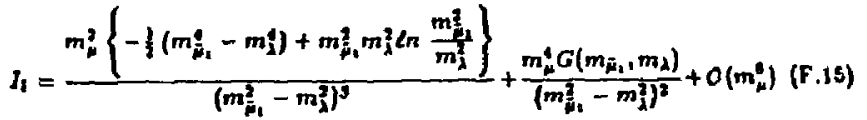


where

$G\left(m_{\tilde{\mu}_{1}}, m_{\lambda}\right)=$

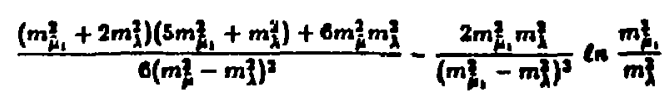

Eqn. (F.11) agrees with Griffole and Mendes" and, with some algebre, Ellis, Hagelin and Nanopoulos".

The contributions of diagrams VI and VII are the tame 4 the bove except that $\bar{\mu}_{1} \rightarrow \tilde{\mu}_{2}$ and the overall additiond fector of -1 coming from $-\sin$ at one of the Yukawa vertices.

Thus the term of $\Delta a_{\mu}$ which is linear in $m_{\mu}$ is

$$
\Delta o_{\mu}^{\text {linaw }}=\frac{a}{2 \pi} \sum_{j=1}^{2} \frac{m_{\mu} m_{\lambda} \sin \phi \cos \phi}{\left(m_{\mu_{j}}^{2}-m_{\lambda}^{2}\right)^{2}}\left[f\left(m_{\mu_{j}}^{1}-m_{\lambda}\right)-m_{\lambda}^{2} m_{j}^{2} \ln \frac{m_{L_{j}}^{2}}{m_{j}^{2}}\right] \text {. }
$$

When $m_{\tilde{\mu}_{i}} \approx m_{\lambda}$ the above formulation would appear to be invalid since $\left|m_{i j}-m_{\lambda}\right|$ might be comparable to $m_{\mu}$ terms which were discarded. If $m_{\mu_{i}}>m_{\mu}$, however, then (F.17) is atill valid and yields

$$
\Delta a_{\mu}\left(m_{\dot{\mu}}=m_{\lambda}\right) \rightarrow-\frac{a}{2 \pi} \frac{m_{\mu}}{m_{\lambda}} \sin \phi \cos \phi .
$$

Equation (F.17) can also be written as

$$
\begin{aligned}
\Delta a_{\mu} & =c\left(f\left(x_{2}\right)-f\left(x_{1}\right)\right) \\
c & =\frac{a}{2 \pi} \frac{m_{\mu}}{m_{\lambda}} \sin \phi \cos \phi \\
f(x) & =\frac{l\left(x^{2}-1\right)-x \ln x}{(x-1)^{3}} \quad f(1)=\frac{1}{6} \\
x_{i} & =m_{i}^{2} / m_{\lambda}^{2} .
\end{aligned}
$$

\section{F.4 A note on AXIal Coupluas}

Grifola and Mendes ariginally conaldered this posibility of a more ceneral vertex coupling of the form

$$
\bar{\nabla}(\boldsymbol{w}+\boldsymbol{s} \boldsymbol{\gamma}) \lambda_{\mu_{i}} .
$$

This in more in keeping with the full electroweal theory. Since

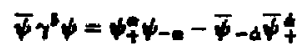

we that thic really implien that $\bar{\nabla}_{+} \lambda$ and $\psi_{-} \lambda$ may couple with unequal coeficients. The relative Yulawe atrengthe of the verticed and a few of the anlient dingrams are Hlustrated in Fig. F.3.

- We nole thet or - en comes from the $\tilde{m}_{R}$ part of $\tilde{\mu}_{1}$ while $g_{V}+g_{A}$ items from the $\tilde{\mu}_{L}$ part of $\tilde{\mu}_{1}$. Thus diagrammatic contributions where the helicity Aips

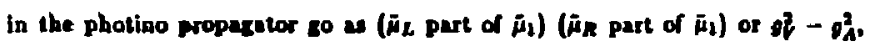
which is like din deos in the aotation which we have adopled. The ather two contributions are Hke $(r-g a)^{2}+(v+g)^{2} \propto g^{2}+g_{\lambda}^{2}$. Thus in this case we would find

$$
\begin{aligned}
& \Delta a_{\mu}=-\frac{\left(Q^{2}-\alpha_{\lambda}^{2}\right)}{\sigma^{2}} \sum_{j=1}^{3}(-1)^{j} \frac{m_{\mu} m_{\lambda}}{\left(m_{i j}^{2}-m_{\lambda}^{2}\right)^{3}}
\end{aligned}
$$

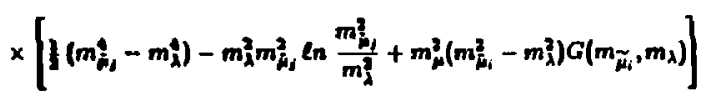

$$
\begin{aligned}
& -\frac{\left(e_{r}^{2}+n_{1}^{2}\right)^{2}}{6 \pi^{2}} \sum_{j=1}^{2} \frac{m_{j}^{2}}{m_{j j}^{2}} \text {. }
\end{aligned}
$$




\section{F.3 SAALL MASS-SPLITTHO}

When $m_{j_{1}} \approx m_{j}$, we may simplify the expression in (F.19).

Lelting

$$
6 m^{2}=m_{\mu_{1}}^{2}-m_{i_{1}}^{2} \quad m_{-}=m_{\tilde{m}_{1.2}}
$$

we find (for $m_{\lambda}<m_{\lambda}$ )

$$
\begin{aligned}
& \Delta 0_{\mu}=-\frac{6 m^{2}}{m_{F}^{2}} \frac{a}{4 \pi} \sin \phi \cos \phi \frac{m_{\lambda} m_{\mu} m}{\left(m_{p}^{2}-m\right)^{4}} \\
& \times\left\{1+\frac{m_{\lambda}^{2}}{m_{i}^{2}}\left[1-\ln \frac{m_{i}^{2}}{m_{2}^{2}}\right]-\frac{m_{\lambda}^{1}}{m_{i}^{!}}\left[5+2 \ln \frac{m_{L}^{l}}{m_{L}^{2}}\right]\right\}
\end{aligned}
$$

or

$$
\Delta a_{\mu}=-\frac{\delta m^{2}}{m_{i}^{2}} \frac{\alpha}{4 x} \sin \phi \cos \& \frac{m_{m}}{m_{1}} p(x)
$$

with

$$
\begin{aligned}
& x=m_{i}^{2} / m_{i}^{2} \\
& p(x)=\frac{x}{(x-1)^{4}}((x+5)(x-1)-2(2 x+1) \ln x) \\
& p(x \rightarrow 0) \rightarrow-4 x \ln x \\
& p(x \rightarrow 1) \rightarrow \frac{1}{6} \\
& p(x \rightarrow \infty)-\frac{1}{x} .
\end{aligned}
$$

When we furthet have $m_{\lambda}<m_{j}($ i.e. $x \geq 1$ ) then

$$
\Delta \Delta_{\mu} \doteq-\frac{a}{4 \pi} \sin \phi \cos \downarrow \frac{m_{\mu} m_{A}}{m_{k}^{2}} \cdot \frac{\delta m^{2}}{m_{k}} \text {. }
$$

\section{F.6 EXTAEMA OF THE LINEAR TERM}

In Chapter 2 we saw that it is the turm linear in $m_{\mu}$ which places the most rizorous limits when compared with the "real world". We may divide $\Delta \sigma_{\mu}$ into

$$
\Delta \dot{a}_{\mu}=\Delta e_{\mu}^{a}-\Delta a_{\mu}^{1} \quad \Delta e_{\mu}^{i}=C J\left(X_{1}\right)
$$

using the notation of (F.10). The function $f(x)$ has been plotted in Fig. G.1

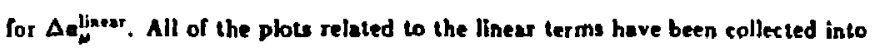
Appendix G. We immediately cee from the form of $J(x)$ that there will be an extremum in $\Delta \mathrm{a}_{\mathrm{a}}^{\mathrm{lim}}=c\left(f\left(x_{3}\right)-f\left(x_{1}\right)\right)$ when $X_{1} \neq x_{1}$. Differentiating to find the maximum $\Delta e_{\mu}^{\text {lin }}$ we find that it occurs when

In $x_{\mathbf{t}}=$

$\frac{y\left[\left(x_{1} y+5\right)\left(x_{1} y-1\right)-2\left(2 x_{1} y+1\right) \ln y\right]\left(x_{1}-1\right)^{4}-\left(x_{1}+5\right)\left(x_{1}-1\right)\left(x_{1} y-1\right)^{4}}{2\left[y\left(2 x_{1} y+1\right)\left(x_{1}-1\right)^{4}-\left(2 x_{1}+1\right]\left(x_{1} y-1\right)^{4}\right]}$

(where $y \neq 1$ when $x_{:} \neq 1$. Here $x_{1}=X_{1}$ )

$$
x_{1}=\frac{m_{m_{1}}^{2}}{m_{2}^{2}} \quad x_{2}=\frac{m_{m_{2}}^{2}}{m_{2}^{2}} \quad y=\frac{x_{3}}{X_{1}}=\frac{m_{w_{2}}^{2}}{m_{E_{1}}^{2}} .
$$

This extremum, a meximum for $\left|\Delta a_{\mu}^{h_{n}}\right|$, we shall call $\Delta a_{\mu}$ man. For a given value of $y$ it occurn at $X_{1}=X_{1}$ ax and wo $\Delta a_{\mu \text { max }}=\Delta a_{\mu \text { max }}(y)$ and $X_{1 \text { mu }}=$

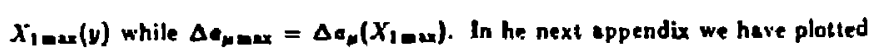
$f(x), \Delta a_{\mu}(X)$ for $y=1.01$ and $y=2, \Delta a_{\mu}=a(y)$ and $X_{1}$ ear $(y)$ for a range of parameters. Discussion takes place in Chapter 2. 


\section{FIGURE CAPTIONS}

1. Vertices and Propagators

(a) Photon-Scaler Muon (Imuon) Vertices.

(b) Photino Yuknwa Vertices.

(c) Photino Propacator illus:ating tipping und non-nipplins propugation.

2. Lowest order contributions to $\Delta a_{\mu}$.

3. Sample Dingrams from $\bar{\phi}\left(\alpha r+\theta_{A} \gamma\right) \lambda \bar{\mu}$ Vertex. (o)
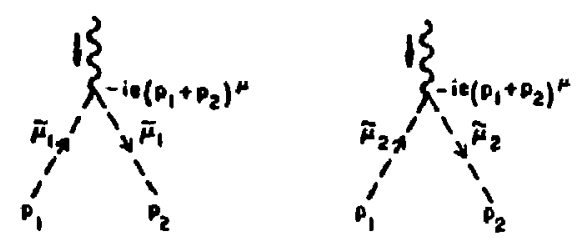

(b)
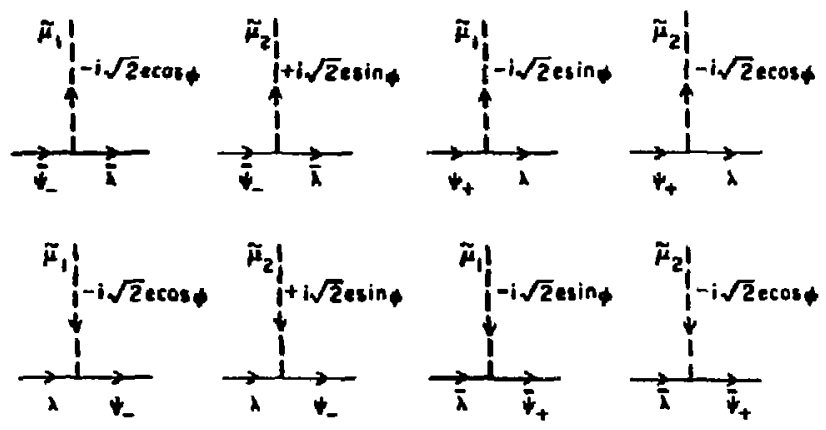

(c)
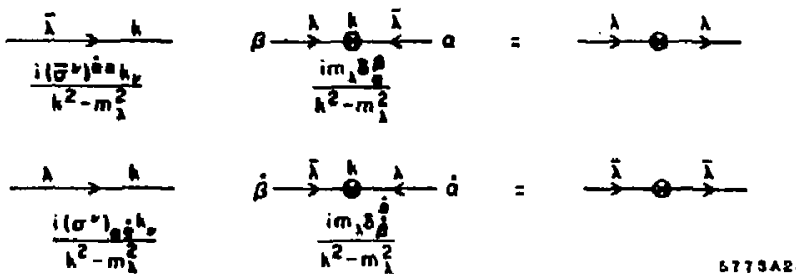

Fig. F.1 

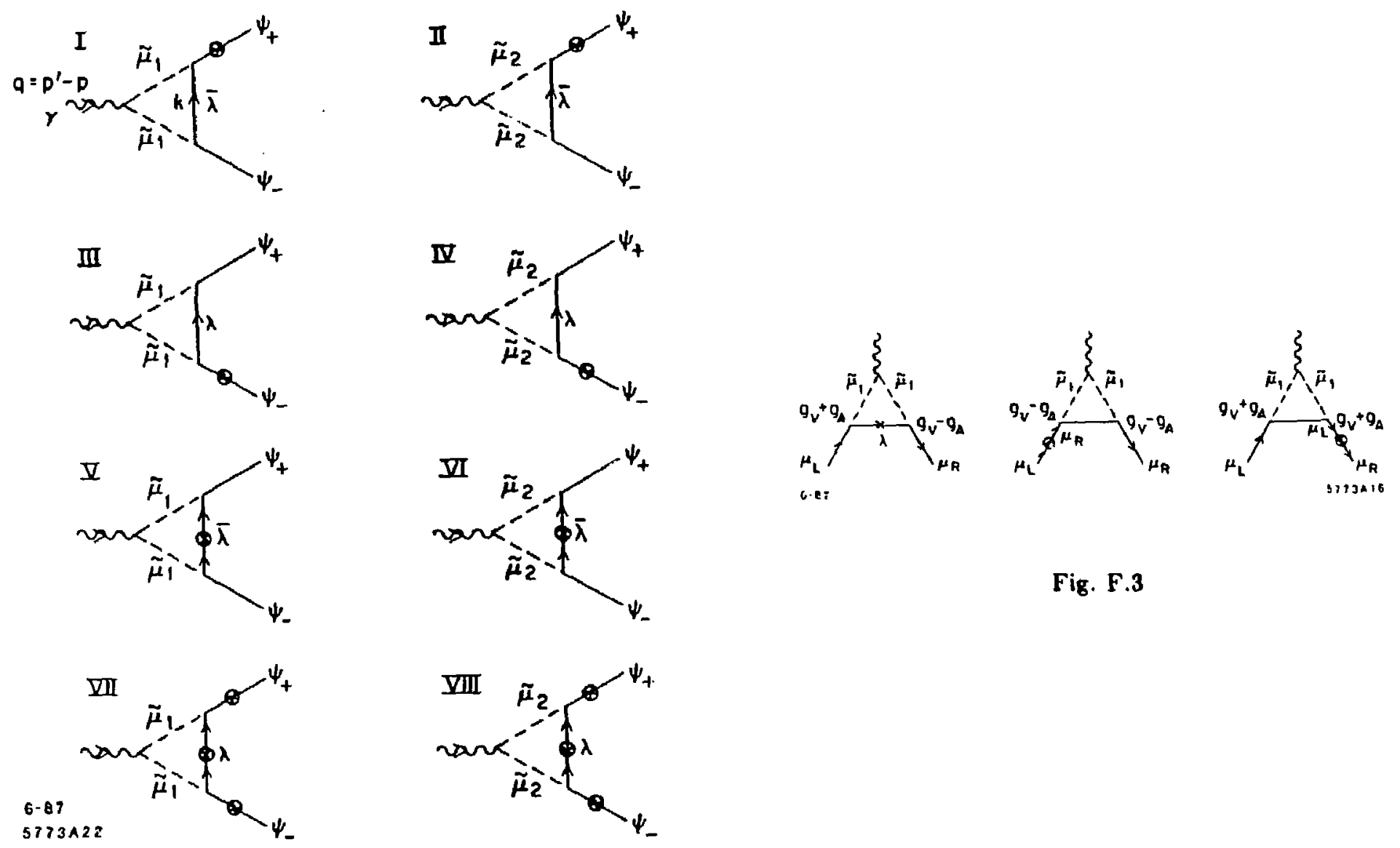

Fig. F.3

Fig. F.2 


\section{APPENDIX G}

Plow Reloted to Anomalow Moment

In this appendix we plot come of the results derived in the previous appendix. In each instance this is for the case of amuon mixing. The slates $\tilde{\mu}_{L}$ and $\tilde{\mu}_{R}$ mix with angle $\$$ to produce the eigenstates $\tilde{\mu}_{1}$ and $\tilde{\mu}_{2}$. Letting

$$
\begin{gathered}
X_{i}=m_{h_{1}}^{2} / m_{\lambda}^{2} \quad i=1,2 \quad y=m_{\mu_{2}}^{2} / m_{\mu_{1}}^{2} \\
C=\frac{a}{2 \pi} \frac{m_{\mu}}{m_{\lambda}} \sin \phi \cos \phi
\end{gathered}
$$

we found that $\Delta a_{\mu}$, the additional contribution to $a_{\mu}$ from euperuymmetric smuon mixing, wes given by

$$
\Delta a_{\mu}=C\left\{/\left(x_{2}\right)-J\left(x_{1}\right)\right\}
$$

where $f(x)$ is given in Eq. (F10).

\section{FIGURE CAPTIONS}

Fic. G.1

$f(x)$ versuk $x$.

Fi.. $\mathbf{G . 2}$

$10^{4} \times t \Delta a_{\mu}$ versus $X_{1}$ for $y=1.01$.

Fi. $\mathbf{G . 3}$

$t \Delta e_{\mu}$ versue $x_{1}$ for $y=2$.

Fis. G.4

$X_{1 \text { max }}(v)$ veraw $y$ whese $X_{1=a x}$ is the value of $X_{1}$ whete $t\left|\Delta a_{i n}\right|$ achieves its extremal value for a tiven $y$.

Fig. G.5

$\mathcal{C}\left(\Delta a_{\mu}^{\text {lin }}\right)_{\text {max }}$ versus $v$ for $y$ ronging from 0 to 10 where $t\left(\Delta a_{\mu}^{l i n}\right)_{\max }$ is the extremal value of $\boldsymbol{t}\left(\Delta \mu_{\mu}^{j}\right)$ cor a siven $y$.

Fis. G.6

$t\left(\Delta e_{j}^{\text {lin }}\right)_{m a x}$ in vicinity of $y=1$. 


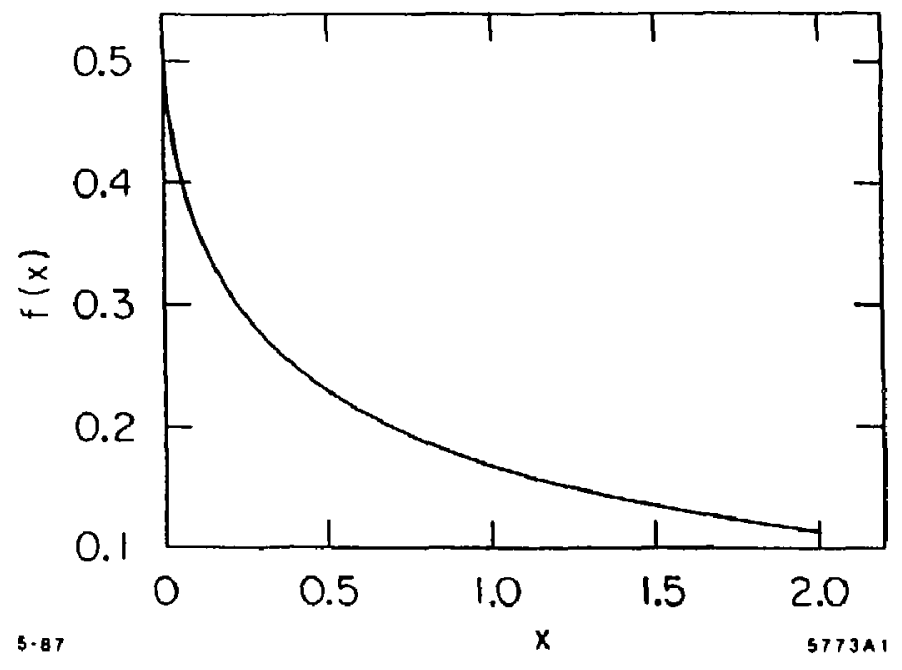

Fig. G.1

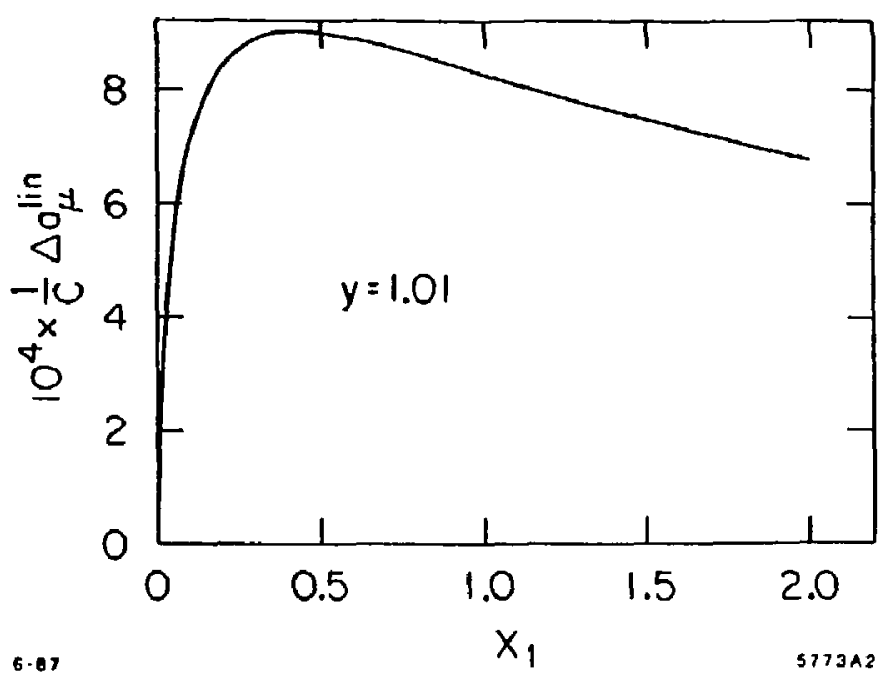

Fig. G.2 


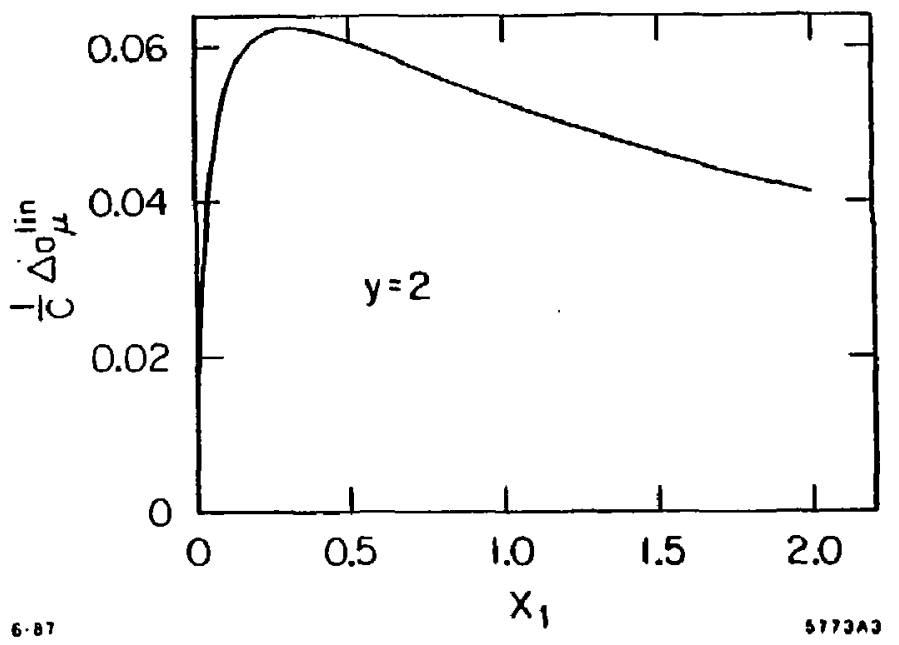

Fig. G.3

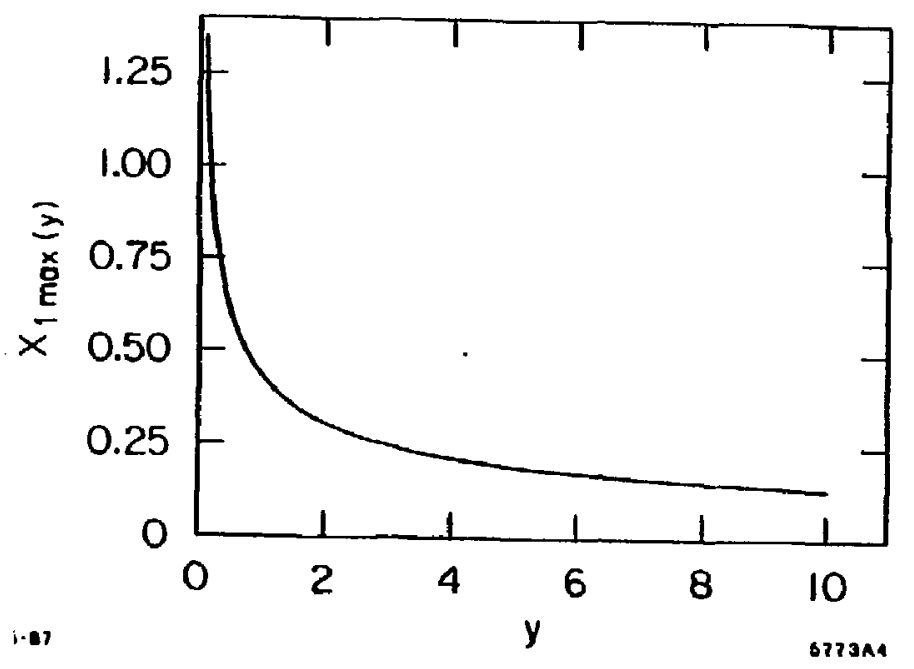

Fig. $\mathbf{G . 4}$ 


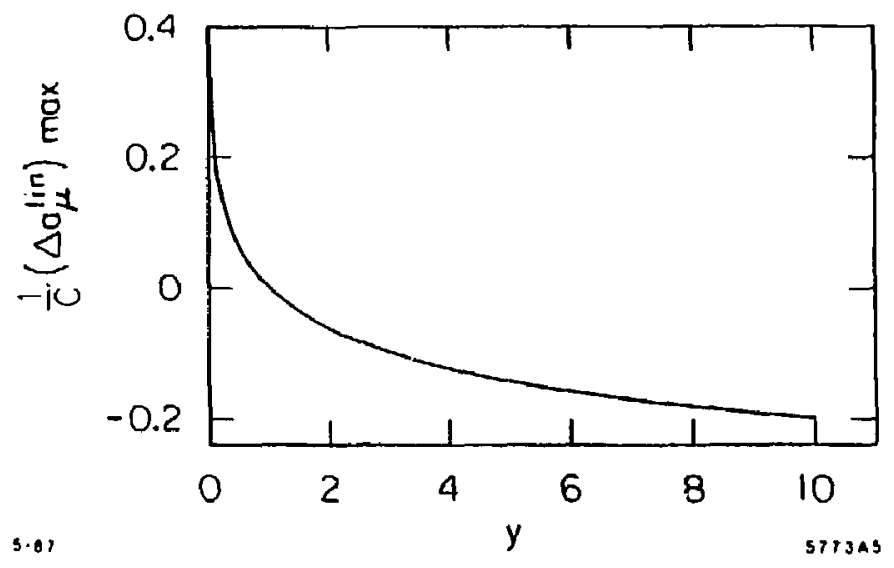

Fig. G.5

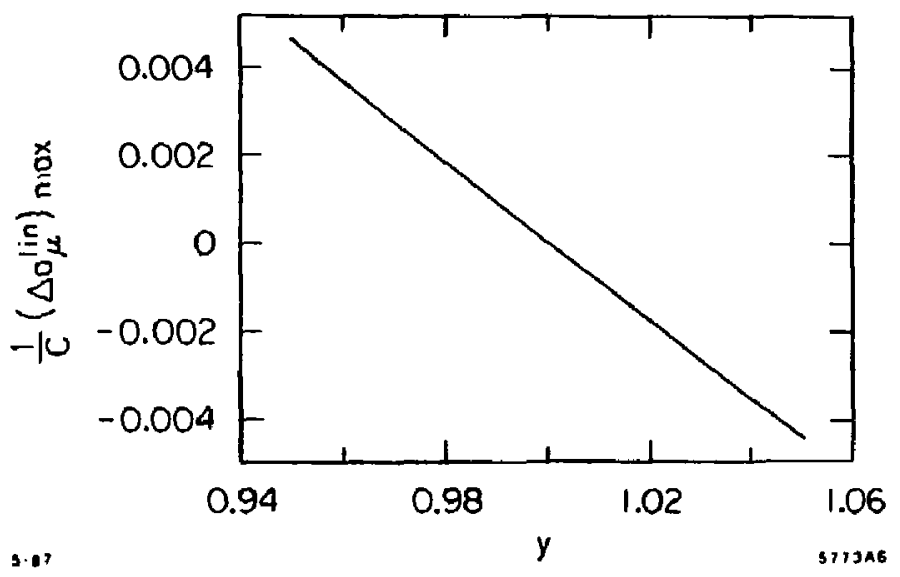

Fig. G.6 


\section{APPENDIX H}

\section{Useful Relationshipe}

In this appendix we gather certain algebraic formulas which are frequently referred to in this paper.

\section{H.1 FeysMaN IntEgRaLs}

A number of "tricks", due 10 Feynman, permit us to reparameterize the momentum integrals which frequently arise in the computation of Feynmen diagrams. A general exprevsion in

$$
\begin{aligned}
& \frac{1}{D_{1}^{n} D_{2} \cdots \cdots D_{N}^{\beta^{N}}}= \\
& \frac{\Gamma\left(p_{1}+p_{2}+\cdots+p_{N}\right)}{\Gamma\left(p_{1}\right) \Gamma\left(p_{2}\right) \cdots \Gamma\left(p_{N}\right)} \int_{0}^{1} d x_{1} \cdots \int_{0}^{1} d x_{N} \frac{x_{1} p_{1}-1_{x_{2}} p_{3}-1 \cdots x_{N} p_{N-1} \delta_{\left(x_{1}+\cdots+x_{N}\right.}+\cdots}{\left(D_{1} x_{1}+\cdots+D_{N} x_{N}\right)^{n_{1}+\cdots+p_{W}}}
\end{aligned}
$$

We will use a slightly different form of this identity." The particular cases of interest are

$$
\begin{aligned}
& \frac{1}{a b}=\int_{0}^{1} d x \frac{1}{|a x+b(1-x)|^{2}} \\
& \frac{1}{a^{2} b}=2 \int_{0}^{1} d x \frac{x}{\mid a x+b(1-x)]^{3}} \\
& \frac{1}{a b c}=2 \int_{0}^{1} d x \int_{0}^{1-x} d y \frac{1}{|a+(b-a) x+(c-a) y|^{3}} \\
& \frac{1}{a^{2} b c}=6 \int_{0}^{1} d x \int_{0}^{1-x} d y \frac{1-x-y}{|c+(b-a) x+(c-a) y|^{4}}
\end{aligned}
$$

These are particularly useful in conjunction wilh

$$
\begin{aligned}
& \int \frac{d^{4} k}{(2 \pi)^{4}} \frac{1}{\left(k^{2}-2 p \cdot t+t-i c\right)^{3}}=\frac{i}{32 \pi^{2}\left(t-p^{2}\right)} \\
& \int \frac{d t}{(2 \pi)^{4}} \frac{k^{n}}{\left(t^{2}-2 p \cdot t+t-i t\right)^{3}}=\frac{j p^{k}}{32 \pi^{2}\left(s-p^{2}\right)} \\
& \int \frac{d k}{(2 x)^{4}} \frac{1}{\left(k^{2}-2 p \cdot k+p-i t\right)^{4}}=\frac{i}{96 x^{2}\left(s-p^{2}\right)^{2}} \\
& \int \frac{d t}{(2 \pi)^{4}} \frac{t^{*}}{\left(k^{2}-2 p \cdot t+t-i t\right)^{4}}=\frac{i p^{4}}{\sin ^{2}\left(x-p^{2}\right)^{2}}
\end{aligned}
$$

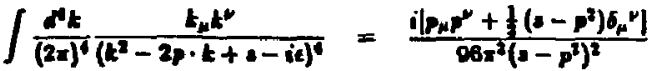

In order to improve cosvergeace we frequently change an incegral of the form (H.2) to (H.3) or (H.4) to (H.S) by introducing an additional parametric integral

$$
\frac{1}{a} \rightarrow-\int_{0}^{\infty} \frac{d t}{(e-t)^{2}}
$$

In paricular if $a=k^{2}-m^{2}$ we introduce the ${ }^{25}$ Pauli-Villare regulstor, $1 \rightarrow \infty$ :

$$
\frac{1}{E^{2}-m^{2}} \rightarrow \frac{1}{E^{2}-m^{2}}-\frac{1}{E^{2}-A^{2}}=-\int_{m^{2}}^{\infty} \frac{d}{\left(k^{2}-t\right)^{2}}
$$

Instead of $x$ and $y$ in equ. (H.7) we frequently find it useful to introduce

$$
\begin{array}{cc}
z \equiv x+y & \Sigma \equiv x-y \\
x=\xi(x+\bar{z}) & y=\frac{\xi}{2}(x-\bar{z})
\end{array}
$$

whence it becomes easy to show that

$$
\int_{0}^{1} d x \int_{0}^{1-x} d y=1 \int_{0}^{1} d x \int_{-1}^{1} d z
$$


and, if the integrand is even in $\bar{E}$,

$$
\int_{0}^{1} d x \int_{0}^{1} d y=\int_{0}^{1} d x \int_{0}^{1} d x
$$

Consider a triangle loop diagram whose legs have moment e $p_{1}$, $p_{2}$ (fermions) and $y=p_{1}+p_{2}$ with masses $m_{1}, m_{2}, m g$ and internal particles whose masses are $M_{1}, M_{8}$ and $M_{3}$. A typical Feynman integral for such a triangle diagram would have $\triangle$ piece which looks like

$$
\mu^{\mu} \sim \int d^{4} k \frac{\left(H_{1}-k\right) \tau^{\mu}\left(\gamma_{2}+\mu\right)}{\left|k^{2}-M_{3}^{2}\left\|\left(p_{1}-k\right)^{2}-M_{i}^{2}\right\|\left(p_{1}+k\right)^{2}-M_{3}^{2}\right|}
$$

and from (H.9), with $A \rightarrow \infty$,

$$
\mu^{N} \sim-\int_{\mu_{q}}^{A^{3}} d t \int d^{4} k \frac{\left(\nu_{1}-\mu\right) \gamma^{\mu}\left(p_{2}+k\right)}{\left|k^{2}-t\right|^{3}\left|\left(p_{1}-k\right)^{2}-M_{1}^{3} \|\left(p_{2}+k\right)^{3}-M_{2}^{2}\right|}
$$

The denominator is of the form $\frac{1}{a^{2} b e}$ so we may use (H.S) to obtain

$$
\begin{aligned}
M^{\mu} \sim- & \int_{M_{3}^{3}}^{h} d t d^{4} k 6 \int_{0}^{l} d x \int_{0}^{1-2} d y \\
& \times \frac{(1-x-\nu)\left(H_{1}-k\right) \gamma^{\mu}\left(H_{2}+k\right)}{\left[\left(k^{2}-t\right)+\left(t-2 p_{1} \cdot k+p_{1}^{3}-M_{1}^{2}\right) x+\left(t+2 p_{2} \cdot k+p_{2}^{2}-M_{3}^{2}\right) y\right]^{4}} .
\end{aligned}
$$

Now, if the fermions with moments $p_{1}$ and $p_{2}$ are on shell, then

$$
p_{i}^{2}=m_{i}^{i} \quad p_{i}^{3}=m_{i}^{3}
$$

and (II.13) becomes

$$
\begin{aligned}
& M^{*}--\int_{A \xi}^{A^{2}} d t d^{4} k 6 \int_{0}^{1} d x \int_{0}^{1+z} d y \\
& \times \frac{(1-x-y]\left(t_{1}-k\right) \gamma^{M}\left(t_{2}+k\right)}{\left[\left(k^{2}-t\right)+\left(t-2 p_{1}+k+m_{1}^{2}-M_{1}^{2}\right) x+\left(l+2 p_{2}-k+m_{1}^{2}-M_{2}^{2}\right) y\right]^{4}} \\
& =-\int_{M / P}^{h^{2}} d t \int^{4} k 6 \int_{0}^{1} d x \int_{0}^{x-x} d y \\
& \times \frac{(1-x-y)\left(y_{3}-\mu_{1}\right)\left(f_{2}+R\right)}{\left[k^{2}-\left(p_{1} x-p_{2} y\right) \cdot k+t(x+y-1)+\left(m_{1}^{4}-M_{1}^{2}\right) x+\left(m_{2}^{3}-M_{2}^{3}\right) y\right]^{4}}
\end{aligned}
$$

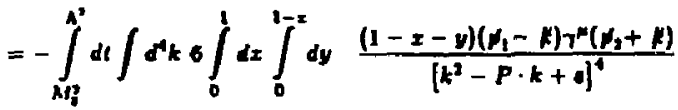

where

$$
\begin{aligned}
& P=p_{1} x-m y \\
& v=m_{1}^{2} x+m_{1}^{2} y-\left(M l_{1}^{2} x+M_{2}^{2} y\right)-t(1-x-y)
\end{aligned}
$$

We could equally we have writhed (H.11) with the find two terms of the denominator in reverse order. Then, when we applied (H.5) with $6 \leftrightarrow c$, instead of getting (11.12) we would have obtain the same expression but with $x \rightarrow y$. Thus we may always interchange $x$ and $y$ in the integrand. Keeping this in mind we apply (H.7) to obtain

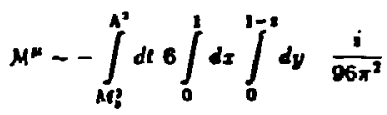

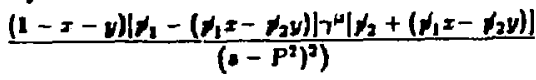

14 
Forgetting the f-term for now we tee that

$$
\begin{aligned}
& P^{2}=p_{2}^{2} x^{2}+p_{2}^{2} y^{2}-2 p_{1} \cdot p_{2} x y \\
& q^{2}=\left(p_{1}+p_{2}\right)^{2}=p_{1}^{2}+p_{2}^{2}+2 p_{1} \cdot p^{2} \\
& \text { so } \quad 2 p_{1} \cdot p_{2}=q^{2}-p_{1}^{2}-p_{2}^{2} \\
& =q^{2}-m_{i}^{2}-m_{2}^{2} \\
& \text { and } P^{3}=m_{1}^{2} x^{2}+m_{i}^{2} y^{2}-\left(q^{2}-m_{i}^{2}-m_{i}^{2}\right) x y \\
& =m^{2}(x+y)^{2}-d^{2} x y \quad \text { when } m_{1}=m_{1} \equiv m \text { (H.LT b) } \\
& 2-P^{2}=\left(m_{1}^{2} x+m_{2}^{2} y\right)(1-x-y)-\left(M_{1}^{2} x+M_{2}^{2} y\right) \\
& -t(1-x-y)+y^{2} x y
\end{aligned}
$$

Specinlizing to the case $m_{1}=m_{2} \equiv m$ and $M_{1}=M_{2} \equiv M_{1}$, for the purposen of this demonstration, we then have

$$
s-P^{2}=m^{2}(x+y)(1-x-y)-M^{2}(x+y)-t(1-x-y)+q^{2} x y
$$

which is symmetric in $x$ and $y$. Using (H.10a) we may wrile this a

$$
-P^{2}=m^{2} z(1-x)-M^{2} x-1(1-x)+\frac{x^{2}}{4}\left(x^{2}-z^{2}\right)
$$

Since we are able to interchange $x+y$ in the integrand and obtain and ldentical expression, we are similarly able to let $\bar{~} \rightarrow-\mathbf{i}$. We may then average these two exprestions. In either case

$$
\begin{aligned}
& x+y \\
& z+-5
\end{aligned}
$$

We will refer to thit as the "b $\leftrightarrow c$ trick". Since the denominator, $\bullet-P^{2}$, is even in of $\bar{z}$, when we perform this averaging we will eliminate all terms in the
Dumerator which are odd in $\bar{z}$. Instrtias (H.10 b) into (H.15) yialds

$$
\begin{aligned}
& \mu^{n} \sim-\int_{\mu}^{4} d t \int_{0}^{1} d x \int_{0}^{1-x} d y \frac{i}{\cos ^{2}}(1-x) \\
& \frac{\mid\left[x\left(t_{2}-H_{1}\right)-E\left(H_{1}+H_{2}\right)+2 f_{1}|\gamma| x\left(H_{1}-H_{2}\right)+E\left(H_{1}+H_{2}\right)-2 f_{2}\right]}{\left(a-P^{2}\right)^{2}}
\end{aligned}
$$

Expanding thic and keppias only even powen of $\Sigma$ gives a numerator term

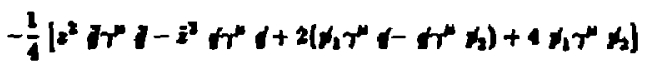

where here

$$
\begin{aligned}
& p^{n}=n_{1}^{\prime \prime}+n^{\prime \prime} \\
& z^{\prime \prime}=x_{i}^{\prime \prime}-p_{h}^{\prime \prime}
\end{aligned}
$$

The above expreasion will be andwiched batween $a\left(p_{1}\right)$ and $\nu(p)$ so that any $f_{1}$ to the bit of $T^{n}$ will geserate a factor of $m_{1}$ vie the Dirac equation and any $f_{3}$ to the right of $q^{n}$ will generate en $m_{2}$. $f m_{1}=m_{2}=m<M$ or $q^{2}$ (which appears in terms not shown) then we can ignore these terms and (H.19 b) becomes

$$
-P^{2} x-\left[(1-z)+M^{2} x+\frac{1}{4} \varphi^{2}\left(\bar{z}^{2}-\varepsilon^{2}\right)\right]
$$

which, when used in (H.21), cives us

$$
\begin{aligned}
& M^{\mu}--\int_{N^{2}}^{d^{2}} d 6 \int_{0}^{1} d x \int_{0}^{1-2} d y \frac{i}{96 x^{3}} \\
& -\frac{f(1-x)\left[x^{2}\right)\left(\gamma-z^{2}\left(\gamma^{\mu} d\right]\right.}{\left(1(1-2)+M^{2} z+3 q^{2}\left(z^{2}-z^{2}\right)\right)^{2}} \text {. }
\end{aligned}
$$


In point of fact, since we are ignoring the external fermion mase lerms,

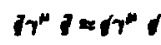

and (H.21) may be written as

$$
\begin{aligned}
& \mu^{\mu} \sim-\int_{M_{3}}^{A^{*}} d t 6 \int_{0}^{1} d x \int_{0}^{1-2} d y \frac{i}{06 \pi^{2}} \\
& -\frac{f(1-x)\left[\left(z^{2}-z^{2}\right)\left(z^{m} d\right]\right.}{\left((1+x)+M^{2} z+\left\{z^{2}\left(z^{2}-z^{2}\right)\right)^{2}\right.} \text {. }
\end{aligned}
$$

\section{H.2 THE GORDON DECOMPOSITION}

The Gordon Decomposition is a useful identity" relating currents of even and odd numbers of sigme (or gamme) matricen. In this cenue it is merely a restatement of the Dirac equation recent into a form which includes a $\sigma^{* N}$ term. Since the statement and proof of this identity may be found in anj number of stundard references, it may usem atrange to devole a major portion of an appendix to it. Applieations of this result are to be found throughout this paper, often in centralized or aty pical forms which we will proceed to derive.

\section{Four-Component Formulations}

Working with Dirae spinors the Gordon Decomposition of the Dirac vector current is generally expressed as

$$
\bar{\psi}\left(p^{\prime}, s^{\prime} \gamma^{\mu} \psi(p, s)=\frac{1}{2 m}\left\{\bar{\psi}\left(p^{\prime}, s^{\prime}\right)\left[\left(p^{\prime}+p\right)^{\mu}+i \sigma^{\mu \nu}\left(p^{\prime}-p\right)_{\nu}\right) \psi(p, s)\right\} \quad\right. \text { (H.26) }
$$

or

$$
\left.\bar{\psi}\left(p^{\prime}, s^{\prime}\right) \gamma^{\mu} \psi(p, s)=\frac{1}{2 m}\left\{\bar{\psi}\left(p^{\prime}, s^{\prime}\right) \mid \bar{p}^{\mu}+i \sigma^{\mu \nu} q v\right) \psi(p, s)\right\}
$$

where, for tymmelry, :e have let

$$
\boldsymbol{\varphi}^{\mu}=p^{\mu \nu}-\boldsymbol{p}^{\mu} \quad \vec{q}^{\mu}=p^{\mu \mu}+p^{\mu}
$$

We use the basic identitie

$$
\begin{aligned}
& \left\{\gamma^{\mu}, \gamma^{\nu}\right\}=2 g^{\mu \nu} \\
& {\left[\gamma^{\mu}, \gamma^{\nu}\right]=-2 i \sigma^{\mu \nu}}
\end{aligned}
$$




$$
\begin{aligned}
A \gamma & =a_{\mu} b^{\nu}\left(\left|\left(\gamma^{\mu}, \gamma^{\nu}\right)+\right|\left|\gamma^{\mu}, \gamma^{\nu}\right|\right)=\left|a_{\mu} b_{\nu}\right| g^{\mu \nu}-2 i \sigma^{\mu \nu} \mid \\
& =a \cdot \omega-i a^{\mu \nu} \omega_{\mu \nu} .
\end{aligned}
$$

The atendard identity in (H.27) conniders only the inutence of a vector current $\left(7^{\mu}\right)$ where the initint and ond apinorial atates are of the eamo type. We wish to extend this to adait the possibility of admixtures of vactor and peeudo vector currents connecting anter of different apecies. We will refor to these as $\psi^{\text {i and }}$ $\psi^{i}$ and consides the vertex $|A+B r j| r^{\prime}$.

We will require a few fdenthies invoiving 7 in addition to (H.29)-(H.31).

$$
\begin{aligned}
& {\left[7, \gamma_{\mu}\right]=0 \quad\left[\gamma, 0^{\mu \nu}\right]=0 \quad \gamma^{k}=\boldsymbol{x}}
\end{aligned}
$$

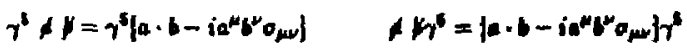

First consider the case where $A=1$ and $B=0$. Let $a^{\prime \prime}$ be any fous-vactor.

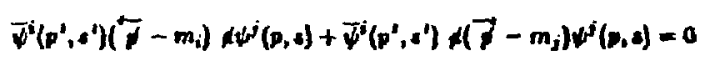

by the Dirac equation. The arrowe alop the momentum oparalors indicale in which direction we intend to operate. This is true for ubltrory c. Vine (H.31) we find that this is equal to

$$
\begin{aligned}
& -\left(m_{i}+m_{j}\right) \bar{\psi}^{\prime}\left(p^{\prime}, s^{\prime}\right) d \psi^{j}(p, e)
\end{aligned}
$$

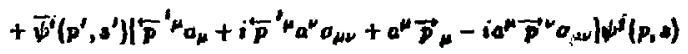

thus

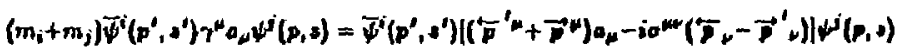

since onv $=-0^{*}$. An this in true for aby on we may writs

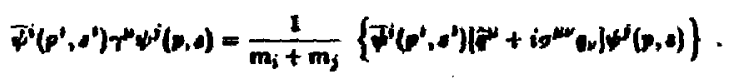

When $i \approx j$ we oblais (H.27), of coures. We now consider the cese where $A=0$ and $B=1$ and repeat the above analyoim

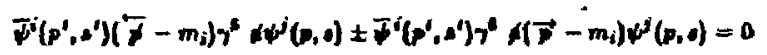

$$
\begin{aligned}
& =-\left(m_{i} \pm m_{j}\right) t^{6}\left(\theta^{\prime}, a^{\prime}\right) \gamma^{6}+\psi^{j}(b, a)
\end{aligned}
$$

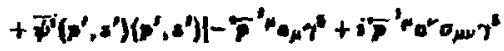

$$
\begin{aligned}
& \left. \pm \tau^{6} \omega^{\mu} \vec{P}_{\mu} \mp i \omega^{\mu} \vec{p}^{*} \gamma^{5} \sigma_{\mu \nu}\right\} \psi^{j}\left(p_{r}+\right)
\end{aligned}
$$

The two different signs give rise to the equlvalent ldentlties:

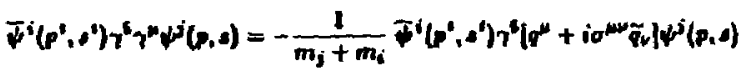

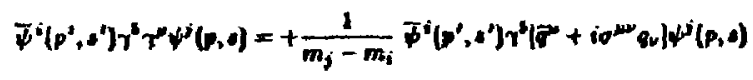

$$
\left(m_{i} \neq m_{i}\right)
$$

These can now be combined with (H.93) to obtain the mote central resulls

$$
\begin{aligned}
& \bar{\psi}^{i}\left(p^{\prime}, s^{\prime}\right)\left|A+B \gamma^{3}\right| \psi^{j}(p, s) \\
& =\frac{1}{m_{i}+m_{j}} \nabla^{i}\left(p^{\prime}, s^{\prime}\right)\left(\left(A F^{\mu}-B q^{\mu} \gamma^{i}\right)+i\left(A q_{r}-B \tilde{q}_{\nu} \gamma^{\sigma}\right) o^{\mu \nu}\right\} \psi^{j}(p, 4)
\end{aligned}
$$




\section{$\Psi^{\prime}\left(p^{\prime}, s^{\prime}\right)\left|A+B \gamma^{\prime}\right| \psi^{\prime}(p, s)$}

$=\bar{\psi}^{\prime}\left(p^{\prime}, v^{\prime}\right)\left[\frac{A}{m_{j}+m_{i}}+\frac{B_{\gamma}}{m_{j}-m_{i}}\right]\left(\hat{q}^{\mu}+i \sigma^{\mu \nu} q_{w}\right) \psi^{j}(p, s) \quad\left(m_{i} \neq m_{j j}\right.$

In either version we recover (H.27) when $A=1, B=9$ and $m_{i}=m_{j}$. Writing

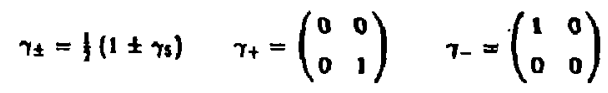

and usine (11.36) we find that

$\left(m_{i}+m_{,}\right) \bar{\psi}^{i}\left(p^{\prime}\right) \gamma \pm \gamma^{\mu} \psi^{j}(p)=$

$\bar{\psi}^{\prime}\left(p^{\prime}\right)\left\{\gamma=p^{\mu \mu}+\gamma \pm p^{\mu \prime}+i \sigma^{\mu \nu}\left|\gamma_{\mp} p^{\prime \nu}-\gamma_{ \pm} p^{\nu}\right|\right\} \psi^{\prime}(p)$.

(H.39)

\section{Twa-Component formulation}

As always, the Weyl spinor formulation of an Identity derived for Dirac spinors ts more involved. We now have a pair of spinors and their conjugates which are related vin the Dirac equation. One posalbility to to take the results of the previous section and expand them out in two-component form. By choosing $A+B \gamma_{s}$ to be $\left\{\left(1 \pm \gamma_{s}\right)\right.$ we will project out the lefi- and right-piecs of the vector current.

Expanding some Dirac epinorial currents into theh two-component subdivi- sion (see Appendix A)

$$
\begin{aligned}
& \bar{\psi} \boldsymbol{\gamma} \psi \boldsymbol{\psi}=\bar{\psi}-\bar{\psi}+ \\
& \overline{\boldsymbol{\psi}} า-\boldsymbol{\psi}=\boldsymbol{\psi}+\boldsymbol{\psi}
\end{aligned}
$$

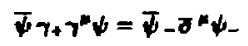

$$
\begin{aligned}
& \bar{\psi} \gamma_{-} \gamma^{\mu} \psi_{\varphi}=\psi_{+} a^{\mu} \bar{\psi}_{+} \\
& \bar{\psi}_{\tau_{+} 0^{\mu v}} v=\bar{\psi}_{-} \boldsymbol{D}^{\mu \nu} \bar{\psi}+ \\
& \bar{\psi} \gamma_{-} \boldsymbol{v}^{\mu \nu} \boldsymbol{\psi}=\psi_{+} 0^{\mu \nu} \psi_{-}
\end{aligned}
$$

Using these with (H.39) results in

$$
\begin{aligned}
& \psi_{+}^{i}\left(p^{\prime}\right) \sigma^{\mu} \bar{\psi}_{+}^{j}(p)= \\
& \left.\frac{1}{m_{i}+m_{j}} \mid \psi_{+}^{i}\left(p^{\prime}\right)\left(p^{\prime \mu}+i \alpha^{\mu \nu} p_{\nu}^{\prime}\right) \psi_{-}^{j}(p)+\bar{\psi}_{-}^{i}\left(p^{\prime}\right)\left(p^{\mu}-i \sigma^{\mu \nu} p_{\nu}\right) \bar{\psi}_{+}^{j}(p)\right\} \\
& \bar{\psi}_{-}^{i}\left(p^{\prime}\right) \sigma^{\mu} \psi_{-}^{j}(p)= \\
& \frac{1}{m_{1}+m_{j}}\left\{\psi_{+}^{i}\left(p^{\prime}\right)\left(p^{\mu \prime}-i \sigma^{\mu \nu} p_{\nu}\right) \psi_{-}(p)+\bar{\psi}_{-}^{i}\left(p^{\prime}\right)\left(p^{\prime \mu}+i \bar{\sigma}^{\mu \nu} p_{\nu}^{\prime}\right) \bar{\psi}_{+}^{j}(p)\right] . \\
& \text { (H.12) }
\end{aligned}
$$

These could equally well have been oblained by working with the Weyl apinors directly. For instence, Etarting with

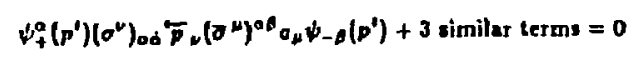

and repeating the stepy leading to (H.33) would lead us to (H.42) directly. It is ofien convenient to use the sum and difference of (H.41) and (H.42) which correspond to decomposing the full $\gamma^{\mu}$ and $\gamma^{\prime} \gamma^{\mu}$ cursents. 
The oum teres is

$\psi_{+}^{i} a^{\mu} \psi_{+}^{j}+\psi_{-}^{i} \sigma^{\mu} \psi_{-}^{j}$

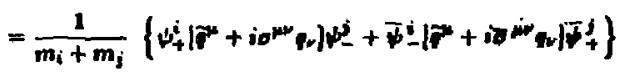

which is, of coure, merely the expantion of (H.33). We cen wes the Dirac equation (section B.1) to put this entirdy in terme of $\bar{\phi}$ - and $\$-$.

$$
\begin{aligned}
& \bar{\psi}\left(p^{\prime}\right)\left[\frac{3 \cdot p^{\prime}}{m_{i}}+\frac{\sigma \cdot p}{m_{j}}\right] \psi^{j}(p) \bar{z}
\end{aligned}
$$

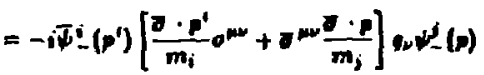

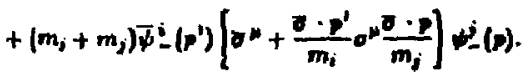

$A$ aimilar expression exisls for $\psi_{+}$and $\bar{\psi}_{+}$( 10 -ahell wa may cancel the opinors from these expressiona.)

\section{A PPENDIX}

The Supenymmetric Lagrangian

1.1 Basics

\section{Superapace and Supertields}

We remind the reader of some fundumental aspects of supersymmetric theories. We extend normal space-time to "auper-space" which includea a number of complex anti-commuting (Grasman) coordinates $\mathcal{A}_{A}, \bar{J}_{0}\left(\bar{G} \equiv U^{\prime}\right)$. Super fields are functions of these superupace coordinater

$$
\phi=\phi\left(x^{n} ; \theta_{10} \delta_{i}\right) \quad \mu=1 \ldots, D \quad \bullet=1, \ldots K
$$

where $D$ in the dimensionally of spuce-time and we refer to this a an " $n=K$ " aupersymmetry. In this paper we will restrict oureelves to $D=4$ and $K=1$ which corresponds to the simpleat aupersymmetry .

We aball take of and to be two-component entf-commuting objects (akin to Weyl spinorel:

$$
u=\left(\begin{array}{l}
\omega_{1} \\
v_{2}
\end{array}\right) \quad J=\left(\begin{array}{l}
J_{1} \\
\nu_{2}
\end{array}\right) .
$$

Thus

$$
\left\{\left(u_{0},\right\}\right)=\left(\overline{0}_{1}, \overline{0}_{j}\right)=\left\{0_{i}, \overline{0}_{j}\right\}=0 \quad i, j=1,2 .
$$

In particular

$$
\theta_{1}=\theta_{2}^{2}=\theta_{1}^{2}=\sigma_{2}^{2}=0 \text {. }
$$


For thort we wite

$$
\theta^{2}=\theta=f e^{a f \theta_{0} \theta_{\theta}=\theta_{1} \theta_{2}}
$$

Note that $\theta^{3}=0=i^{3}$ and likewise for higher powers of $t$.

\section{Supertranslormations and the Supersymmetry Generatore}

We examine a superfield under the action of an infinitesimal translation in superspace ("supertransformation")

$$
0 \rightarrow 0+6 \quad J \rightarrow J+2 \quad x^{*} \rightarrow x^{*}+b x^{*}
$$

where end $z$ are infinilesimal, two-component, anti-commuting c-numbers. We desire to construct $6 x^{4}$ from the anti-commuting coordinates and $\delta$ subject to the constraint that $x^{\mu}$ must be a real coordinate which commuter, not anticommules. Since $x \psi$, the product of two Grassman objects, to a commuting object we must construct $\delta I^{\mu}$ from produzts of $f^{\prime}$ and 6 's.

The two simplest transformations which retain the Lorents atructure are

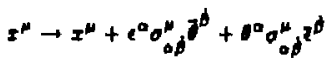

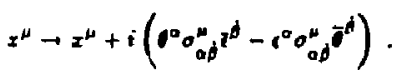

Note that using eop and at these may be recast into equivalent forms. The second equation in (J.6) is the traditional choice. These forms follow betause on (end 8 ") are the only dimensionless constants with a single Lorentz index. Note that here $, t, t$ are e-numbers and we are dealing with rigid (alobal) supersymmetry. In a local theory (l.e. uppergravity) $t=c(x)$ and $x=x(x)$ but $c \neq c\left(\theta_{i}\right)$.
Representitus these supertransformations by ( $\phi$ is auperbeld)

$$
\phi \rightarrow+6, \phi
$$

where

$$
\delta=\ell Q+i \bar{Q}
$$

we can find the supersymmetry zenerators $Q$ and $\bar{Q}$.

$$
\begin{aligned}
& \phi\left(x^{\mu}, \theta, \bar{\phi}\right) \rightarrow \phi+\delta_{1} \phi=\phi\left(\theta+c, \bar{\phi}+\tau_{1} x^{\mu}+i\left|\theta_{0}^{\mu} 2-c^{\mu}\right\rangle \mid\right) \\
& =\phi+i \frac{\partial \phi}{\partial \phi}+i \frac{\partial \phi}{\partial \bar{\theta}}+i \frac{\partial \phi}{\partial x^{\nu}}\left(\rho^{\nu} \mathrm{z}-10^{\mu} \bar{\theta}\right) \\
& =\phi+e\left(\frac{\partial}{\partial \theta}-i \sigma^{\mu \nu} \frac{\partial}{\partial x^{\mu}}\right)+\tau\left(\frac{\partial}{\partial \bar{\nu}}-i \sigma^{\mu} \frac{\partial}{\partial x^{\nu}}\right)
\end{aligned}
$$

where the final minus sign comes from anticommuting $x$ through $6 \sigma^{\mu}$. Nole that

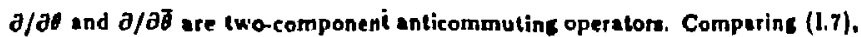
(1.8) and (1.9) we have

$$
\begin{aligned}
& Q=\frac{\partial}{\partial \theta}-i \sigma^{M} \frac{\partial}{\partial z^{\mu}} \\
& \bar{Q}=\frac{\partial}{\partial D}-i \theta \sigma^{\mu} \frac{\partial}{\partial x^{\mu}}
\end{aligned}
$$

Note that the detinition of $\bar{Q}$ differs by a sign when compared with those of

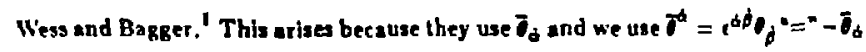
where " $=$ " meang "which has the numerical maznilude".

Summarizing, under the superrymmetry transformation $\delta_{\text {e }}$

$$
\begin{aligned}
& \iota \rightarrow 0+c \\
& \bar{c} \rightarrow \bar{t}+\tau \\
& x^{\mu} \rightarrow x^{\mu}+i\left(0 a^{\mu} z-\cos ^{\mu} \bar{\nu}\right)
\end{aligned}
$$


the superfield $\$\left(x^{n}, 0,0\right)$ traniform a

$$
\begin{aligned}
& \phi \rightarrow+\infty \phi \\
& \text { 4. }=2 Q+20 \\
& Q=\frac{\partial}{\partial \theta}-i \alpha^{N} \bar{\partial} \frac{\partial}{\partial x^{*}} \\
& \bar{Q}=\frac{\partial}{\partial \partial}-i \alpha_{\alpha}^{\alpha} \frac{\partial}{\partial x^{\mu}} \text {. }
\end{aligned}
$$

\section{Relationship with Hamiltonian}

It can be shown that $\left|\sigma_{t}, \sigma_{t}\right| \sim\left(\cos ^{\mu} \bar{\varepsilon}-\xi_{0} \alpha t\right) a_{\mu}$ and so two successive nupartymmetry transformations fead to a Lotentz transformation (in a locel theory this will necessilate a torsional term). In a sense the supernymmetry tranaformations ere "squase rooks" of Lorentz trensformations. In particulas $\left(Q_{c}, \bar{Q}_{j}\right)=20_{0 j}^{\prime \prime} P_{\mu}$ Multiplying by ( $\left.0^{0}\right)^{\text {pa }}$ (i.e. Cating the trace) lende to

$$
Q_{1} \bar{Q}_{1}+Q_{2} \bar{Q}_{2}+\text { A.c. }_{2}=P_{0}=n
$$

and so the Hamiltonian is sum of the squares of the superaymmelry generators and is always positive ceml-definite. This $Q$ is like the "square root of $X^{*}$.

\section{Covarint Derivative}

We desire a nupersyrametric derivative which cammutes with $9 Q$ and $\bar{Q} \bar{Q}$ wo that if $\phi \rightarrow \phi+\delta_{d} \phi$ then $D \phi \rightarrow D \phi+\delta_{1} D \phi$ (i.e. if $\phi$ transforms as a supesfield then so does $D \phi$ ). It is easily checked that if we let

$$
\begin{aligned}
& D=\frac{\partial}{\partial \theta}+i \sigma^{\mu} \bar{\partial} \frac{\partial}{\partial x^{\mu}} \\
& \bar{D}=\frac{\partial}{\partial D^{\prime}}+i \sigma_{0}^{\mu} \frac{\partial}{\partial x^{\mu}}
\end{aligned}
$$

then $\{\in, E D \mid=\{Q, D\}=0$ and similerly

$$
[Q, \bar{D}\}=[\bar{Q}, \bar{D}]=\{\bar{Q}, D)=0 .
$$

Note that $D$ is like $Q$ with a change of aign. (1.15) impties that $\delta_{1} D \phi=D \varepsilon_{c} \phi$ using the definition of 4, in (1.12).

\section{Diferentizstion and lneteration}

Differentiation and integration over Grawmen vatiables is lartely a matter of definition. We define ( $\xi$ is a single component)

$$
\frac{\partial}{\partial \xi} A=0 \quad \frac{\partial}{\partial \xi} \xi=1
$$

when $\boldsymbol{A}$ is independent of $\boldsymbol{\varepsilon}$ and could be commuting, anti-commuting, or a combination,

Note that

$$
\frac{\partial}{\partial E} A E= \begin{cases}A & \text { If } A \text { is commuting } \\ -A & \text { if } A \text { is enti-commuting }\end{cases}
$$

For integrations we invole three axlom

(i) Linearity

$$
\int(A F(\xi)+B G(\xi)) d \xi=A \int F(\xi) d \xi+B \int G(\xi) d \xi .
$$

Note that $\int d \xi$ is a definite integral over "all values of $\xi^{\prime \prime}$ and that $\int A d \xi=$ $-\int d \xi A$ if $A$ is anti-commuting.

(ii) Shifting variable of integration

$$
\int F(\xi+x) d \xi=\int F(\xi) d \xi \text {. }
$$

(iii) If $x \epsilon=0 \quad \forall \quad x$ then $\varepsilon=0$. 
Consider:

$\int(\xi+x) d \xi=\int \xi d \xi+\int x d \xi$ by linearity

$\int(\varepsilon+x) d \xi=\int \xi d \varepsilon$ by shitting

$\Rightarrow \int x d \xi=0$

$=x \int d \xi \quad \forall x$

$\Rightarrow \int d \xi=0$.

Since $f^{3}=0$ ( $f$ a aingle-componen $l$ object) any function of 6 has a trivial expand. sion of the form

$$
F(\xi)=A+B \xi
$$

and the most general function is a linear Inaction.

Then

$$
\int F(\xi) d \xi=\int A d \xi+B \int t d \xi=B \int t d \epsilon
$$

and unless we want integration to be totally trivial (all integrals vanish) we must have $\int \xi d \xi \neq 0$. For simplicity we select

$$
\int \varepsilon d \xi \equiv 1
$$

We see that the rules for differentiation and integration are identical with $\partial_{\rho}=$ $\int d f$ acting like projection operators, projecting out only those terms with 2 f.

10.
Multiple integrals work the same way (keeping track of anti-connuting d\& $d x$, etc.). Thus for a two-component object $\theta=\left(\begin{array}{ll}O_{1} & O_{1}\end{array}\right)^{\top}$

$$
\int e^{2} d^{2} \theta=\int \theta_{1} d e_{1} \int e_{1} d \theta_{2}=1
$$

and all other combinations vanish. Note again that $\partial F / \partial \theta^{2} \equiv \int F d \theta^{2}$.

Component Fiesta.

Let us consider the expansion of a cuperfield $\phi(x, 0,7)$ where 1,0 are twa component anti-commuling (coordinates) parameters

$$
\begin{aligned}
& \left.\phi(x, 0, \pi)=a(x)+\left(D_{4}+\bar{\psi}_{2}\right)+\left(x_{2}\right)+0 ; \pi\right)
\end{aligned}
$$

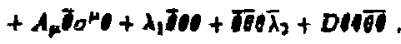

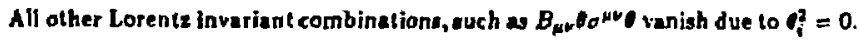
Domplepel is proportional to 1030 and 20 is not included. If $\phi$ is to be real then $\therefore A_{n}$ and $D$ are real and $\psi_{1}=\lambda_{2}, a_{1}=a_{g}$ and $\lambda_{1}=\lambda_{2}$.

This represents many degrees of freedom. Just as a massless cause field, Á, has two, not four, physical decrees of freedom. To reduce the multitude of nonphysical degrees of freedom a number of "superzaver-like" constraints are used. Although it is not readily apparent, a hallmark of supesaymmetsic theories is that the number of physical fermionic and boapnic degrees of freedom are equal. First it should be realized from (1.24) that any function of a super field is another superfield.

This can be explicitly checked by testing that it transforms as a euperfield under $\delta_{\rho}$. For instance, since $D_{\phi}$ and $D_{\phi}$ are tuperfields, $\phi^{3} D_{\phi e^{i d}} D_{\phi}$ would also be superfield. For our constraint we could choose $/(\phi)=0$ which would be

Io 
- manifestly supenymmetric constraint. In practice the two which are uevally chosen are $D \bar{\phi}=0$ and $\phi=\phi^{\circ}$.

\section{Scalar Superfielde}

We call superfields satisfying

$$
\bar{D} \phi \mathbf{0}
$$

chiral (or scalar) superfields and their conjugates

$$
D \phi^{\prime}=0
$$

enti-chiral superfields, or superfield of the opposite chirality. These equalions may be trivially colved. Recalling that

$$
\bar{D}_{\alpha}=\frac{\partial}{\partial J^{d}}+i \cdot \sigma_{\partial \alpha}^{\mu} \frac{\partial}{\partial x^{\mu}}
$$

we note that

$$
\bar{D}_{\dot{\alpha}}\left(x^{\mu}+i \sigma^{\mu} \bar{D}\right)=\bar{D}_{\dot{\alpha}} \varphi^{\alpha}=0
$$

and therefore

$$
\phi=\phi\left(x^{\mu}+i \theta \sigma^{\mu} \overline{0}, \theta\right)
$$

is $\star$ solution of $\bar{D} \phi=0$. This follows since

$$
\frac{\partial}{\partial \bar{\theta}} \phi\left(x^{\mu}+i\left(\sigma^{\mu} \bar{\theta}, \theta\right)=-i \theta \sigma^{\mu} \frac{\partial \phi}{\partial x}\right.
$$

(remember to anti-commute $\partial / \partial \bar{U}$ pait $\theta$ ) and

$$
\begin{aligned}
& \frac{\partial}{\partial x^{\mu}} \|\left(x^{\mu}+i 0^{m}, 0\right)=\phi^{\prime} \\
& \left(\frac{\partial}{\partial \phi}+i \sigma^{\mu} \frac{\partial}{\partial x^{\mu}}\right) \phi=0=D_{\phi} \text {. }
\end{aligned}
$$

Similarly $D \bar{\phi}=0$ hes colution

$$
\phi=\bar{U}\left(x^{n}-i \| o^{m} \bar{D}, \bar{\theta}\right)
$$

is an "unti-chiral" field.

Since we can wrile

$$
\begin{aligned}
& \phi=\phi(y, 0) \\
& \bar{\phi}=\bar{\psi}\left(y^{t}, \overline{0}\right) \\
& v^{m}=x^{m}+i 0 \sigma^{n} \overline{0}
\end{aligned}
$$

we see that is not an explicit function of $\overline{0}$ and that $\bar{b}$ not an explicit function of a. This in the principle reason that thase particular constraints were chowen. The expension of $\phi(y, 0)$ is now simplified considerably

$$
\downarrow=A(v)+\sqrt{20} \psi(v)+t^{2} F(v)
$$

where the $\sqrt{2}$ is chosen to make the closure of the algebre simple. Since $\phi(y, 0)$ is independent of $\overline{-}$ we see that any functlon of $\phi(y, 0)$ will aloo be a chiral cuperfeld, asruming that there is no $\overline{0}$ explicitly in the function definition, because $f(\phi)$ will also be independent of $\bar{b}$. Note that $A(v)$ is a scalar field and $\psi(v)$ a spinor. This closure is easily ween by considering l'ie effect of the supersymmetry ceneraton $Q$ and $\bar{Q}$ on $A_{1} \psi$ and $F$ which are called the "cornponent fields" of the ouperfield $\phi$. 
Irensformation of Sceler Superfield Components

$$
\begin{aligned}
& \delta_{l} A=(\bullet Q+\tau \bar{Q}) A \\
& \delta_{t} \psi=(\ell Q+\imath \bar{Q}) \psi \\
& \delta_{t} F=(\bullet Q+\tau \bar{Q}) F .
\end{aligned}
$$

Now consider the dimensionality of the quantities involved. Since $Q \sim \sqrt{H} \sim \sqrt{E} \Rightarrow$

$$
|Q|=m^{1 / 2}
$$

Similarly

$$
|\theta|=m^{-1 / 2} \quad|d \theta|=m^{1 / 2} \quad|A|=m \quad|\psi|=m^{3 / 2} \quad|F|=m^{2} \quad|Q|=m^{1 / 2}
$$

In constructing a supermultiplet with a left-handed Weyi zpinot $\$$ we might try $Q A \sim \psi$ on dimensional grounds and since $|Q \psi|=m^{3}$ we coutd invent a state with dimension $m^{2}$ of we can have $Q \psi \sim \partial_{\mu} A$. In general when we operale wlth $Q$ we will obtain a state of higher dimension by $\mathrm{m}^{1 / 2}$ or the derivative of a alate of lower dimension. It can be shown that $\bar{Q}$ adds $\mid$ unit of right-handed helicity while $Q$ adds \& unit of left-handed helicity (helicity here meano $o_{2}$ ). As always adding $j=\frac{1}{2}$ may result in slates of $j_{z} \pm \frac{1}{3}$ corresponding to adding $\mid \frac{1}{2}, \frac{1}{2}$ ) or $\mid \$,-\xi)$. Thus operating with $\delta_{4}$ resulta in both states which have been increased and decreased by $j=\boldsymbol{l}$. Since we are considering a multiplet with a left-handed spin we take $\bar{Q} A=0$ (since it would give a right-handed Weyl spinor). Take

$$
Q A-\sqrt{2} \psi
$$

(The $\sqrt{2}$ is conventional.) Similarly

$$
Q \psi=\sqrt{2} F \quad \bar{\psi} \psi=i \sqrt{2} \sigma^{*} \partial_{\mu} A
$$

Since there is no field with $\boldsymbol{\theta}^{3}$ coefficient $F$ had better transform entirely into W's. (Also $F \sim Q^{2} A$ and so $Q F=0$ since $Q^{3}=0$ beine enti-commuting) thus

$$
Q F=0 \quad \bar{Q} F=i \sqrt{2} \partial_{\mu} \circ \phi
$$

or

$$
\begin{aligned}
& \delta_{1} A=\sqrt{2} t \psi \\
& \delta_{c} \psi=i \sqrt{2} o^{\mu} 1 \partial_{\mu} A+\sqrt{2} \mathrm{c} F \\
& \delta_{c} F=i \sqrt{2} i \sigma^{\nu} \partial_{\mu} \psi=\partial_{\mu}\left(i \sqrt{2} 2 \sigma^{\mu} \psi\right) .
\end{aligned}
$$

Note that $\delta_{t} F=$ lotal divergence. This is of crifical wignificance and is always true of the "top-tnost" component field of a superfeld (i.e. that which is multiplied by the most powers of $v$ 's and $\bar{i} \cdot \mathrm{s})$. The reason $b$ that if we use the $F$-lerm as - Legrangian density then $L=\int d x F$ will transform under a oupersymmetric shift $\delta_{l}$ by $\sim \int \partial_{\mu} \psi \sim \int \psi \cdot d S$ which is a surfuce term at inf nity and vanishes for lopologically trivial systems (such as all that will be consiciered here). Thus $\delta_{1} L=0$ and the Lagrangian will be isvariant under supersymmetry Gransformations. More on this in Section 1.2.

\section{Yeclor Supcrfields}

The previous sections introduced the bealar or chiral superfield which contains a Weyl spinor, a scalar and an object "F called en euxiliary feld. This was subject to the constraint $\bar{D}_{\phi}=0$. Here we introduce the multiplet which contains 
gouge Gelds. The constraint used in that the superfield in seal. We anw from (I.24) and the oubsequent discussion that the expansion of such a superfield into its component fields is

$$
\begin{aligned}
& V=\Delta(x)+\bar{\partial} \psi+\bar{\nu} \theta+\nu^{2} a+\bar{\partial}^{2} a^{\prime}+\nu^{\mu} \bar{\partial} A_{\mu}
\end{aligned}
$$

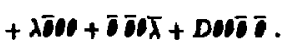

Any superfield of the form $\bar{\phi}$ is a real superfield $(\bar{\phi}=\phi)$ and similarly any termu like $\phi+\bar{\phi}$ in a Lagrangian will be real. In particular $\phi$ coutd be a ecalar superfield.

\section{Super Gauge Transformationa}

In order to expose the physical degrees of freedom of the vestor euperfield, $V$, we will have to foreshadow our discussion of Lagrangian construction of the next section.

The kinetic term of a supersymmetric Lagrangian can be written a $\left(d^{4} 0 \equiv d^{2} \theta d^{2} \bar{D}\right)$

$$
L=\int d^{d} 0 \bar{\gamma}\left(y^{1}, \bar{D}\right)+(y, 0)
$$

where

$$
y^{\mu}=x^{\mu}+i l 0^{\mu \bar{C}} \quad y^{\mu t}=x^{\mu}-i 10^{\mu \bar{\theta}}
$$

recalling that

$$
\bar{D} \phi=0 \quad D \bar{\phi}=0 .
$$

This may ceem like a strange kinetic term since it has no derivativea in it! (One might expect $\int \mathcal{N} \bar{D} \bar{D} D$ or some such similar expression.) Remember that $\phi$ and $\bar{\phi}$ are at difjerent poinls in superspace $\left(\mp^{*} \pm i_{0} \mu \bar{D}\right)$ and so we have a difference inctead of a derivative.

Consider an Abelian theory for now. In a standerd theory unatter fielde transform under the group rotation

$$
\downarrow \rightarrow e^{\wedge(x)} \downarrow
$$

where we have absotbed the charge into the definition of $\Delta(x)$. When $\Delta(x)=\Lambda$, i.t. the rotation is rigid (ebobal), the kinetic term $C_{K J N}=\bar{\psi}, \psi$ is invariant under (I.42). When $\mathbf{L}(x)$ is spatially dependent

$$
\bar{\psi} \nu \psi \rightarrow \bar{\psi} e^{-\Lambda(x)} p\left|e^{i(x)} \psi\right|=\bar{\psi} \phi \psi+i \bar{\psi}(\rho \Lambda(x)) \psi
$$

To compensate we make the atandard "minimal subatitution"

$$
\partial^{\mu} \rightarrow D^{\mu}=\partial^{\mu}+i c A^{\mu}
$$

where $A^{\mu}$ is a new field, called a gauge field, subject to the transformation

$$
A^{\mu} \rightarrow A^{\mu}-\partial^{\mu} \Delta(x)
$$

when $\psi$ is subject to $(1,42)$. "D" th the "coverient derivative". The "covariant" Lagrangian (j.e. invariant under the gauge transformation (I.44)) b

$$
\mathcal{L}_{\boldsymbol{K} J N}=\bar{\psi} \not \psi=\bar{\psi}(\boldsymbol{p}+\boldsymbol{i c} \boldsymbol{\alpha}) \psi
$$

transiorms as

$$
\begin{aligned}
& \bar{\psi} p \psi \rightarrow \bar{\psi} e^{-A(x)}(p+i \varepsilon+i e p \Delta(x)) e^{i \Lambda(x)} \psi \\
& =\bar{\psi}(\rho+i \varphi) \psi \\
& =\bar{\nabla} D \text {. }
\end{aligned}
$$


We desire a timilat realization for a supersymmetric version of an Abelian couge theory, Let us promole $\mathbf{A}(x)$ to be a chiral (scaler) atperfield

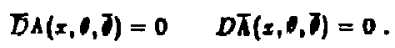

The matter field is also a scalar superfield, f.e. $\psi$ th replaced by $\phi(x, \ell, \bar{\phi})$ and $\bar{\psi}$ by $\bar{\partial}(x, \varphi, \bar{\theta})$. The kinelic term $(1,39)$ becomes

$$
\bar{\phi} \phi \rightarrow \bar{\phi}\left(y^{\dagger}, \bar{J}\right) e^{-i \pi(v 1, \bar{T})} e^{i h(x, n)} \phi(y, 0) \neq \bar{\phi}
$$

where we have used (1.30). We can amend this in the same fachion as before by introducine a (vector) gauge superfield:

$$
C_{K I N} \rightarrow \bar{\beta} e^{*}
$$

where

$$
v \rightarrow V+i(\pi-\Delta)
$$

and so

$$
\begin{aligned}
\bar{\phi} e^{v_{\phi}} & \rightarrow \bar{\phi} e^{-i \pi_{e}(i \bar{\lambda}+V-i \Lambda)_{e} i \Lambda_{\phi}} \\
& =\bar{\phi} e^{v_{\phi}} .
\end{aligned}
$$

The non-Abelian ease is similar except that

$$
\phi \rightarrow e^{i t \cdot A}, \quad \bar{\phi} \rightarrow e^{-i \pi, T^{* \ell}}
$$

and the gauge transformation take the highly non-linear form

$$
e^{v} \rightarrow e^{+i \bar{\lambda}_{0} T^{* 1}} e^{V} e^{-i T \cdot A_{1}} .
$$

Note that in such - tronstormation we must mointoin the constraint on the ouperfields, that it chiral superfield remain chiral and veclor (real) superfields remain co. Recalling from the section on chiral superfields that any function of chiral superficlde is itself a chiral superfield (and similarly for anti-chiral fielda) we see that $e^{i M(x)}$ is chiral and so is $e^{i h(0)}$ wo that the ocaler natute is retained under this transformation. From (1.49), since $\bar{\Delta}=8$ !, we see that the vector nature of $\boldsymbol{V}$ is also retained.

Functions of chiral fields, $\phi_{i}$ with $\bar{D}_{\dot{\varphi}_{i}}-0$, we chital because

$$
\bar{D} F\left(\phi_{i}\right)=\frac{\partial F}{\partial \phi_{i}} \bar{D}_{\phi_{i}}=0
$$

and similarly $D F\left(\bar{\delta}_{i}\right)=0$. Products of ehiral and anti-chiral superfielde ore, in eeneral, neither ehiral nor anti.ehiral.

\section{Wess-Zumino Gaure}

W'e are going to make use of our gauge freedom in the celection of a particular $A(y, 0)$ to reduce the degrees of freedom (i.e. component seldi) in $V$ as much as possible. From (1.30) and (I.31)

$$
\begin{aligned}
& \downarrow=\tilde{\psi}(y)+\sqrt{2} 0 v(y)+\theta^{2} F(y) \\
& y=x+i \theta \sigma \bar{\tau}
\end{aligned}
$$

substituting and expanding about $x=v$ as a power meries allows us to express $\phi$ in terms of $x^{4}$ :

$$
\begin{aligned}
& \phi(x, \theta, d)=\bar{\psi}(x)+\sqrt{2} \theta \psi(x)+\theta^{\prime} F(x)+i \phi \theta^{\mu} \nu \partial_{\omega} \bar{\psi}(x) \\
& \left.-\frac{i}{\sqrt{2}} \rho^{2} \partial_{\mu} \nu(x) \sigma^{\mu}\right\rangle+\frac{1}{4} \rho^{2} \partial^{2} \partial_{\mu} \partial^{\nu} \tilde{v}(x)
\end{aligned}
$$

with a similar expression for $\bar{\phi}$ (the $\bar{\psi}$ is a bosonic field fwe formerly called it 
$A(x) \mid)$ wheres, from (I.3.)

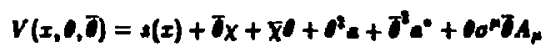

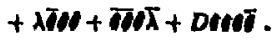

Thus for the gauge transformution $V \rightarrow V+i(\bar{\Lambda}-\Lambda)$ we can retect $\overline{\boldsymbol{\psi}}_{\Delta}+\overline{\bar{\psi}}_{\Delta}$ (i.e.

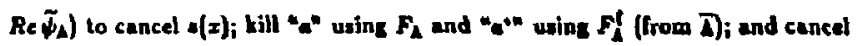
of $X$ with $\psi_{L}$ (the $-\Delta^{*}$ mears "from expanding $A(x, 0, \tilde{0})$ a we did $\phi(x, 0, \overline{0})$ in

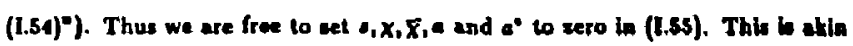
to using the gauge $A_{0}=0$ in QED. We are teft with the selatively simple set of component telds:

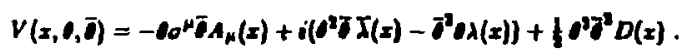

We have altered the coeficients to conform with the definitions used in Weas and Bazzer. This cancellation can be seen from writing out the transformations of the componentr explicitly. For instance

$$
s(x) \rightarrow \Delta(x)+\tilde{\psi}_{\Lambda}(x)+\tilde{\psi}_{\Delta}(x)
$$

which leads to our choice of setting $x(x)$ to zero.

This special choice of geuge in called the Wess-Zumloo Gauge. We ahall use it henceforth at all times. By working in a partirular gauge our cakculations will lose manifest supersymmetry invariance.

We heve not exhausted our gauge freedom yet. Since $R e \tilde{\psi}_{\mathrm{L}}$ wat used to cancel $(x)$ we atill have $I m \tilde{\psi}_{\Delta}$ to play with. This implements the standard $U(1)$ gauge transformations (this is all for the Abelian case):

$$
A_{p} \rightarrow A_{p}+\delta_{p}\left(I_{m} \tilde{\phi}_{h}\right)
$$

We are kft with a gauge vector bonos $A_{\mu}$, an essociated Weyl gauge fermion $(\lambda)$ and an auxiliery seld which transforme tnto e total derivative (just as $F$ did) under a supensymmetry traniformation $C_{i}$.

Aside from eliminating wo many non-physical felds, the Wess-Zumino gauge alo poserses the ubeful property of being of quadratic or higher powers in $0, \bar{\nabla}$ in all of its components. For this reaton $V^{3}=0$ and any function of $V$ terminatea after only 3 terms. In perticular

$$
e^{v}=1+v+1 v^{2}
$$

- wekome simplification in the kinetic lerm $\mathcal{C}=\bar{\phi}_{e} \boldsymbol{v} \phi$. 


\subsection{ANATOMY OF a LAGRANGIAN}

In the previous action we have alablished the machinery to construel supersymmetric Legrenglens.

\section{Supersymmetry Invarience}

In addition to all the invariance propertiea we normally expect from a Lacrangian (gauge, Lorent ) we additionally require that $\mathcal{C}$ be invariant under oupereymmetry tsansformations. If $\mathcal{E}$ is a Lagrantan denalty then the full Lagranglan, $L_{1}$ is obtained by integrating $C$ over all non-timelite coordinalet

$$
L=\int d^{3} x d^{4} \in C\left(x^{\mu}, 1, d\right)
$$

Since $\int d l_{i}=0$ and $\int U_{i} d l_{i}=1$ we see thal (I.59) has no anth-commuting character left $(L=L(I))$ and that $(I .50)$ merely projects out the $d^{2} 7^{2}\left(E 0^{-1}\right)$ eomponent.

When we have only sealar fields, $\phi(v, 0)$ and $\bar{\phi}\left(y^{\dagger}, \bar{\gamma}\right)$, es term in a Lagrangian (and no terms involving both) we see that

$$
L=\int d^{3} y\left[\int d^{2} \cup F(\phi)+\int d^{2} D F^{\prime}(\bar{\phi})\right]
$$

where (bince $\boldsymbol{l}=0$ ) $L$ is now manifestly real and we have used the assumed translational property of the differentialu $d^{3} x=d^{3} y$. For vector muperfields we must use (1.59)

$$
L=\int d^{3} x \int d^{2} 0\left(F(V)+F^{\prime}(V) \mid\right.
$$

and no conjugation of $V$ is needed since $V$ le real.
Superpotentials

The term which corresponds to the potential in en ordinary feld theory is the superpotential. Deroted $W\left[\phi_{1}\right]$ it is a function of the chiral (but not antichiral) superfields in the theory and is thus itself a chiral ouperfield. It eppears in $C$ as

$$
C_{\text {POT }}=\int|\boldsymbol{| \omega}| d^{2} U+\text { h.e. }
$$

where h.e. $=\int W \cdot[\vec{j}|d\rangle$ ensurea a real Lazrangian. Such terms are responsible for masses and Yukwa couplings. This is known as the "Fo ector since the highest (most $1 \cdot c$ ) component fields is of the "F" type (ser (1.31)).

From (I.33) we have $|\phi|=m$ and $\left|d^{2} \theta\right|=m$ so that the most general renor. malizable term of the type (1.62) is

$$
W|\phi|=C+\lambda_{i} \phi_{i}+m_{i j} \phi_{i} \phi_{j}+\rho_{i j}\left\langle\phi_{1} \phi_{j} \phi_{k}\right. \text {. }
$$

We will itnore $C$ which could only play a role in supergravity. Note that there is no quartic term so one might suppose that four boson couplings cannot aecur. Since the " $F$ " term always appears without a derivative in $\mathcal{C}$ (once it has been expanded into its component fields) we can t-ivially obtain its equation of motion via Lagrange's equations

$$
\frac{\delta C}{\delta F}=\partial_{\mu} \frac{\sigma C}{\delta\left(\partial F / \partial x^{\nu}\right)}=0
$$

When $F$ is colved lor and resubstituted into $\mathcal{C}$ we might obtain quartic acalar component field couplings (once the othet terms in $C$ have been added). Nate that in (L.63) these may be many different scalar felds. 


\section{Why we need more Hiry bocons}

The Hicte-seala interactions which generate quark manes orjzinate in the cubic terms. It is because we can only use $\phi$, and not $\bar{\phi}$, that the superaymonetric etandard model requires two Hige doublets. In the standard model Lagrangian we have

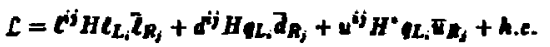

where $c^{i j}$ is diagonal and $(H)=v$. The supersymmetric verulon would be

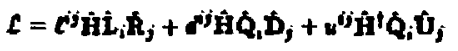

and alnce $\hat{\mathbf{H}}^{\mathbf{I}}=\dot{\mathbf{H}}^{\mathbf{T}}$ cannot appear in $\boldsymbol{W}$ we must define aceond $\hat{\mathbf{H}}, \hat{\mathbf{H}}_{2}$, with the electroweak quantum numbers of ht.

\section{Components of the Superpotential}

In terms of component felds ( $\tilde{\phi}$ ecalux, $\psi$ a lefk-banded Wey\} spinor, $F$ : bosonic auxiliery):

$$
\begin{aligned}
& \phi_{i}(v, v)=\tilde{\psi}_{i}(y)+\sqrt{2} \psi_{i}(y)+\omega F_{i}(y) \\
& \phi_{i} \phi_{j}=\bar{\psi}_{i}(y) \tilde{\psi}_{i}(y)+\sqrt{2}|| \psi_{i} \tilde{\psi}_{j}+\tilde{\psi}_{i} \psi_{j}|+\nu| \tilde{\psi}_{i} F_{j}+\tilde{\psi}_{j} F_{i}-\psi_{i} \psi_{j} \mid \\
& \phi_{i} \phi_{j} \phi_{k}=\tilde{\psi}_{i} \tilde{\psi}_{j} \tilde{\psi}_{k}+\sqrt{2} \epsilon\left|\psi_{i} \tilde{\psi}_{j} \tilde{\psi}_{k}+\tilde{\psi}_{i} \psi_{j} \tilde{\psi}_{k}+\tilde{\psi}_{i} \tilde{\psi}_{j} \phi_{k}\right| \\
& \left.+\omega \mid F_{i} \tilde{\psi}_{j} \tilde{\psi}_{k}+\tilde{\psi}_{i} F_{j} \tilde{\psi}_{k}+\tilde{\psi}_{i} \tilde{\psi}_{j} F_{k}-\psi_{i} \psi_{j} \tilde{\psi}_{k}-\psi_{j} \psi_{k} \tilde{\psi}_{i}-\psi_{k} \psi_{i} \tilde{\psi}_{j}\right]
\end{aligned}
$$

obtained by simple multiplication ignoring $0^{3}$ or highet terms. When we take $\int d^{2} l$ of these we project out the 11 (" $\left.F^{\prime \prime}\right)$ term. The $\sqrt{2}$ is in ketping with Weas and Bayzer notution. For

$W\left|\phi_{i}\right|=\lambda_{i} \phi_{i}+m_{i j} \phi_{i} \phi_{j}+\phi_{i j k} \phi_{i} \phi_{j} \phi_{k}$

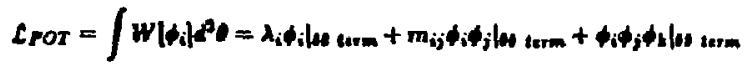

$$
=\lambda_{i} F_{i}+m_{i j}\left|\tilde{h}_{i} F_{j}+\tilde{\psi}_{j} F_{i}-\psi_{i} \omega_{j}\right|
$$$$
+g_{i j k}\left|F_{i} \tilde{\psi}_{j} \tilde{\psi}_{k}+\tilde{\psi}_{i} F_{j} \tilde{\psi}_{k}+\tilde{\psi}_{i} \tilde{\psi}_{i} F_{k}-\phi_{i} \psi_{j} \tilde{\psi}_{k}-\psi_{j} \psi_{k} \tilde{\psi}_{i}-\psi_{k} \psi_{i} \tilde{\psi}_{j}\right|
$$

If $m_{i j}$ and $g_{i j k}$ are symmatric then we can write this a.

$$
c=\lambda_{i} F_{i}+1 m_{i j}\left(\tilde{\psi}_{i} F_{j}-\psi_{i} \psi_{j}\right)+\frac{1}{3} \theta_{i j k}\left(F_{i} \tilde{\psi}_{j} \tilde{\psi}_{k}-\psi_{i} \psi_{j} \tilde{\psi}_{k}\right) .
$$

Nole that, for the $\operatorname{lerm} W|\phi|=\left\langle\phi_{j} \ldots \phi_{N}\right.$, that the $\theta^{a}($ i.e. $* w)$ coeficient will be

$\tilde{\psi}_{1} \tilde{\psi}_{2} \ldots \tilde{\psi}_{N-1} F_{N}+\tilde{\psi}_{1} \tilde{\psi}_{2} \ldots \tilde{\psi}_{N-2} F_{N-1} \tilde{\psi}_{N}+\cdots+F_{1} \tilde{\psi}_{2} \tilde{\psi}_{2} \ldots \tilde{\psi}_{N}$

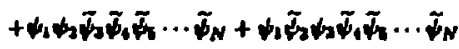

$$
+\cdots+\tilde{\phi}_{1} \tilde{\psi}_{2} \tilde{\psi}_{3} \ldots \tilde{\psi}_{N-2} \psi_{N-1} \psi_{N}
$$

which can be written as

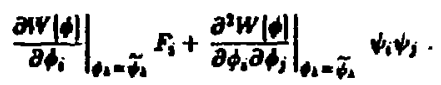

In particular for $\phi_{i}=\phi_{i} V_{i}, j$, l.e. $|W| \phi \mid=\phi^{N}$ we have $\theta^{2}$ coefficient

$$
\begin{aligned}
& N \tilde{\psi}^{N-1} F+N(N-1) \tilde{\psi}^{N-1} \psi^{2} \\
& =W^{\prime} F+\psi^{\prime \prime} \psi \psi
\end{aligned}
$$

evaluated at $\phi=\tilde{y}$. 
$A$ more convenient notation might be

$$
c_{\operatorname{por}}(1 V)=w_{i j}(\tilde{\psi}) F_{i}+W_{i j}(\tilde{\psi}) \psi_{i} \psi_{j}
$$

whese $W_{i}(\tilde{\psi})$ means $\sigma W\left[\phi_{1}, \ldots, \phi_{N}\right] / \partial \phi_{i}$ evaluated at $\phi_{1}=\tilde{\psi}_{1}, \ldots, \phi_{N}=\tilde{\psi}_{k}$.

\section{Solving for $\boldsymbol{F}$}

We may expand the kinc in in (1.10) by using (t.54) and its conjugate. We find that the of coefficient $c \quad-, 7) \phi_{j}(2,0, \bar{J})$ a

$$
\begin{aligned}
& \left.z_{i}(x, 0,7) \phi_{j}(x, 0, \lambda)\right|_{1 \pi}=F_{j}^{\prime} F_{j}+\frac{1}{4}\left[\tilde{\psi}_{i}^{\prime} \square \tilde{\psi}_{j}+\tilde{\psi}_{j}, \square \tilde{\psi}_{i}\right] \\
& -\mid\left(\partial_{\mu} \tilde{\psi}_{i}^{4}\right)\left(\partial^{\mu} \bar{\psi}_{j}\right)+\frac{i}{2}\left[\left(\partial_{\mu} \bar{\psi}\right) \partial^{\mu} \psi_{j}-\bar{\psi}_{j} \delta^{\mu} \partial_{\mu} \psi_{j}\right]
\end{aligned}
$$

writine (thus far)

$$
\left.\mathcal{C}=\mathcal{C}_{K I N}+\mathcal{C}_{\text {POT }}=\int \boldsymbol{\beta}_{i \phi_{1}} d^{4} \theta+\int w\left|\phi_{i}\right| d^{2} \theta+\int w^{\prime}\left|\bar{\delta}_{i}\right| d^{2}\right\rangle
$$

and integrating $L=\int d^{3} \times C$ by parte to convert the $\left(\partial_{\mu} \tilde{\psi}_{i}^{*}\right)\left(\partial^{\mu} \tilde{\psi}_{i}\right)$ term in $(1.74)$ to the form $\tilde{w}_{i} 0 \tilde{\psi}_{1,}$ we oblain (using (1.69))

$$
\begin{aligned}
& \mathcal{L}=i\left(\partial_{\mu} \bar{\psi}_{i}\right) \partial^{*} \psi_{i}+\bar{v}_{i} 0 \overline{\psi_{1}}+F_{i}^{*} F_{i} \\
& +\left[m_{i j}\left(\tilde{\psi}_{1} F_{j}-1 \psi_{i} \psi_{j}\right)+g_{1 j k}\left(\tilde{\psi}_{i} \tilde{\psi}_{i} F_{k}-\psi_{i} \psi_{j} \tilde{\psi}_{k}\right)+\lambda_{i} F_{i}+h_{. e .}\right] .
\end{aligned}
$$

By using (I.61) (or Its conjugate) for the equation of motion of $F$

$$
\frac{\partial C}{\partial F_{i}}=F_{i}+\lambda_{k}+m_{i k} \tilde{\psi}_{i}+y_{i j k} \tilde{\psi}_{i} \tilde{\psi}_{j}=0
$$

or

$$
F_{i}=-\left(\lambda_{k}+m_{11} \tilde{\psi}_{i}+g_{i j k} \tilde{\psi}_{i} \tilde{\psi}_{j}\right) .
$$

Now examining (1.63) (with $C=0$ ) we see that

$$
F_{i}=-\left.\frac{\partial|v| \phi \mid)}{\partial \phi_{i}}\right|_{\lambda_{1}=\tilde{W}_{i}}
$$

Consequently from (1.71)-(1.73)

$$
\begin{aligned}
C_{\text {POT }} & =-\sum_{i}\left|W_{i}(\tilde{\psi})\right|^{2}+\sum_{i j} W_{i j}(\tilde{\psi})+\text { h.e. } \\
& =-\sum_{i}\left|F_{i}\right|^{2}+\sum_{i j} W_{i j}(\tilde{\psi})+\text { h.e. }
\end{aligned}
$$

By using (1.78) in (1.76) we ean eliminate $F$ and $F^{\bullet}$ and plece $\mathcal{C}$ in the form

$$
\mathcal{L}=i \partial_{\mu} \psi_{i} \sigma^{\mu} \psi_{i}+\tilde{\psi}_{i} \square \tilde{\psi}_{i}
$$

$$
-\left(\left\{m_{i k} \psi_{i} w_{k}+q_{i j k} \psi_{i} \psi_{j} \tilde{\psi}_{k}+h . e\right)-\nu\left(\tilde{\psi}, \tilde{\psi}^{\prime}\right)\right.
$$

where the polential $v$ is

$$
v=F_{i} F_{i}
$$

From (1.78) we see that $v$ will provide a mass lerm for $\tilde{\psi}_{i}$ (a acalar) of

$$
\begin{aligned}
& m_{j}=\sum_{i} m_{i k} m_{i k} \tilde{\psi}_{i} \tilde{\psi}_{i}+\left(\sum_{j k} g_{i j l} \lambda_{i} \tilde{\psi}_{i} \tilde{\psi}_{j}+\text { h.c. }\right) \\
& m_{i}=\tilde{\psi}^{\prime} m m^{1} \tilde{\psi}+\left(\tilde{\psi}^{T}\left|g_{k} \lambda_{i}\right| \tilde{\psi}+\text { h.c. }\right)
\end{aligned}
$$

where the second line was written in matrix form with $\left(\tilde{\psi}_{i}\right)$ a column vector, $\tilde{\psi}^{T}=\left(\tilde{\psi}_{1} \tilde{\psi}_{2} \ldots \tilde{\psi}_{m}\right)$ and $m_{1} g_{2}$ matricen. 
Nole that since $D_{a}=D_{a}=0$, where a is a constant, that $D(\phi+a)=0$ is

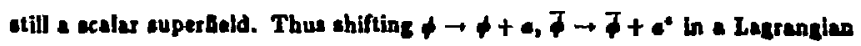
yields enother perfectly valid Lugrangian.' For this reason it is often pousible to eliminate the $\lambda_{i} p_{i}$ term from $W\left|p_{i}\right|$. If that is done then (I.83) bacomes

$$
m_{j}=\tilde{T}|m|^{1} \tilde{\nabla} \quad\left(\lambda_{i}=0\right)
$$

and from (I.a1)

$$
m_{\psi}=1 \psi^{\top} m \psi+\text { h.e. }
$$

and we see an explieit aymmetry between the fermion $\left(\psi_{,} \psi^{\prime}\right)$ and boson $\left(\tilde{\phi}, \tilde{\phi}^{\circ}\right)$ masses. In particular when there is only one ecalar field, $\$$, present with components $\bar{\psi}$ and $\psi$

$$
-L_{\text {max }}=m^{2} \tilde{\psi}^{\bullet} \tilde{\psi}+\mid m \psi \psi+1 m \psi^{\bullet} \psi^{\bullet}
$$

When there are multiple $\phi_{j},(1.84)$ and (1.85) lead to non-diagonal man termi in $\mathcal{L}$. Both may be imultaneously diagonalized by an orthogonal rotation $\psi_{i} \rightarrow o_{i j} \psi_{j i} \tilde{\psi}_{i} \rightarrow o_{i j} \tilde{\psi}_{j}$, i.e. the superfielde may be mixed $\phi_{i} \rightarrow o_{i j} \phi_{j}$ with

$M_{D}=O^{r}$ mo. Note that the fermion must terms are Majorane. Additional Dirac mass terms can bi ablained via the Hices mechenism in the usual woy or vil supersymmetry-breaking .

\section{R-Parity}

R-parity is an additive quantum number that we frequently impose to further restrict our class of theories. It may formerly be written as a global U(1) cymmetry of $\mathcal{C}$ (cee references 1,3 and 39.$)$ It may alos be writlen an (in lawer cenerality)

$$
R=(-1)^{2 J+2 E+L}
$$

where $J, B$, and $L$ are the spin, baryon number and kepton number of a particle. It is also true that

$$
R=-1 \text { for superpurtoer }
$$

$$
R=+1 \text { for ordinary matter. }
$$

In a renarmalizable Lagranginn, inveriance under $A$ may be broken by the terms ${ }^{40}$

$$
\phi_{i} \text { or } \phi_{i} \phi_{j}(i \neq j)
$$

Thit bot ausprising a $\mathcal{C}=\int \lambda \phi_{i} d e$ contains a term which would require that a angle aupenymmetric atate be crented. Conservation of $\boldsymbol{R}$-parity implies that supernymatric atales must be created in pain and consequently the lighteat supereymmetric particle is atable. For most ecalar superfields the opinor term ( $\psi$ ) ib mundane matter and the ccalar $(\tilde{\psi})$ is the euperpartner. The cole exception is the Higs: auperbield where scalar component feld in the normal Hizgs boson $H$ (i.e. $\tilde{\psi} \equiv H)$ and whose fermionic partnes bas odd $R$-parity $(\psi \equiv \tilde{H})$. This explains haw = term weh as sijt $\phi_{i} \phi_{j} \tilde{w}_{\mathbf{k}}$ can be invarient under $R$-perity. If $\phi_{k}=\dot{H}_{k,}$ so that $\tilde{\psi}_{k}=H_{k}$, and $\psi_{i}=Q_{i}=\left(\dot{j}_{i}\right.$ while $\psi_{j}=\bar{d}_{j}$ or $\dot{\alpha}_{j}$ we have rormal Yukwwe-type Higss term where each Geld has $R=+1$. If only * single scalar field of the $\phi_{i}$ is present in the theory then $a$ term like $9 \phi_{i}^{3}$ ewould violate R-parity (as well as lepton number and churge). 


\section{Comments on the Auxiliory Fields}

We heve reen that the component field of highest dimensionality ( $F$ for scalar superfields; $D$ for vector superfields) is an auriliary field, that bappears with no derivative couplings in $\mathcal{C}$. Such felds are non-dynamic (b.e. do not propasute) and, vie their equations of motion $(\partial C / \partial F=0)$, provide a constraint on the other fields. The question arises as to who we bother Including such Gelds at all. (This womewhat akin to aking why we bother including a aigme field in the linewr sigme model when we are going lo solve for it in producing the non-linear sigme model).

We have teen that $\boldsymbol{F}$ is extremely convenient in characterizing the potential enerzy term (V) when a zealar ouperpolential is added. A more fundamental reason its In the fact that an uabrokes superaymmetric theory must have an equal number of fermionte and boonic degreet of fteedom. Indeed the supersymmetry algebra can be derived from this atatement ${ }^{10}$ (plus a few assumptions and require. ments wuh as closure). Looking back at Eqa. (1.32). (1.31) ance (1.35) we may ask why, given the superfield componento $A$ (a scalas, called $\tilde{\psi}$ elsewhere) and $\psi$ (weft-handed Weyl spinor), we could not simply ciose the algebra with $Q \downarrow=0$ ? (i.e. ake 6 ar $t J$ one-component objecte wo that $\phi(y, 0)=A(y)+\sqrt{2} \varphi d(y)$ and $Q . \bar{Q}$ would also be one-component anti-commuting operalors with $Q^{2}=\bar{Q}^{2}=0$.)

Indeed in supersymmetric $(N=1)$ quantum mechenice this can be done

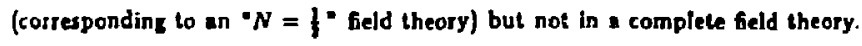
Consider a free field theory of massless particles. Ob. shell, with $A$ an $?$ obeying their equations of motion $\left(\square A=0 ; \sigma^{m} \partial_{\mu} \psi=0\right), \forall$ has one degree of freedom (one helicily atate), as does $A$, and all is right with the univeree. In a field theory $A$ and $\downarrow$ must be able to propagate off-shell. Then $\downarrow$ would have two degrees of freedom (just as if it were mustive) but $A$ atill only hat one. The solvition is to introduce an additional bosonic degree of freedom, $\boldsymbol{F}$, off-shell which has no on-shell dectee of freedom. Such a atate (remember there is no superpolential term $W$ in this free theory) must have the equation of motion $F=0$. To oblain this from $\delta \delta / \delta F=\left(\partial / \partial_{\mu}\right)\left(\delta C / \delta\left(\partial^{\mu} F\right)\right)$ requirea that $\partial_{\mu} F$ not appear and that $F^{n}$ appear with $n>1$. Assuming only terms of quadratic order or less in the Gield Jor cur free Lazrangian we see that $F$ appeare only in the term $\mid F^{2}$ (and so cannol propagate or interact.) By dimensional counting we see that $|F|=m^{2}$. The invariant action now assumes the form

$$
S=\int d^{4} x\left\{-\xi\left(\partial_{\mu} A\right)^{2}-\left\{\psi^{1} \omega^{\mu} \partial_{\mu} \psi+\left\{F^{2}\right\}\right.\right.
$$

Anolhet way of looking at this is that without $F(1.37)$ would become (droppins the $i$ and $\sqrt{2}$ ):

$$
\sigma_{2} A=1 \psi \quad \sigma_{1} \psi=\sigma^{\mu}, \partial_{\mu} A
$$

then

$$
\begin{aligned}
& \left|\delta_{1,}, \delta_{1,}\right| A=2 \epsilon_{2} \sigma^{\mu} c_{1} \partial_{\mu} A \\
& \left|\delta_{(1,}, \delta_{1,}\right| \psi=2 \varepsilon_{3} \sigma^{\mu} c_{1} \partial_{\mu} \psi-c_{2} \sigma^{\mu} z_{1} \delta_{\nu} \sigma^{\mu} \partial_{\nu} \psi
\end{aligned}
$$

end, on-shell, $\sigma^{H} \partial_{\mu} \psi=0$ so that we have a closed complete super-algebra. Off shell we need $\boldsymbol{F}$ with

$$
\delta_{c} \psi=\sigma^{\mu} z \partial_{\mu} A+F \quad \delta_{l} F=\epsilon \sigma^{\mu} \partial_{\mu} \psi
$$

which will cancel the $\sigma^{*} \partial_{\mu} \psi$ term in (1.90). 
The esme argumento may be applied to vector auperkelds, In a free, mankes theory we have a gauge boeon with two degrees of freedom (transverse polarisetiona) and a Marane gauge fermion with two degrees of frendom. Or shell the vector boson gains a longitudinal polarisation and the furmba he four degres of freedom, wa, egain, snother bosonic dezree of fteedom $t$ required. Thus the $D$-field term $1 D^{2}$ i born. $D$, $F$, trensform into a cotel derivalive of the fermion feld $\lambda$.

$$
G_{1} D=2 \sigma^{\mu} \partial_{\mu} \lambda \quad G_{T} D=\cos ^{\mu} \partial_{\mu} \lambda
$$

\section{Super Field Strenth}

Moving on to the gauge sector we desire to find an analogue of the fild atrength tensor, $F_{\mu w}$ of a etandard feld theory. We shall eroin stert whih $A$ bellan theories.

To motiva.s our construction we consider that, bo atendard Beld theory

$$
\left.F_{\mu \nu}=-i \mid D_{\mu}, D_{\nu}\right)=a_{\mu} A_{\nu}-\partial_{\mu} A_{\mu}+i\left[A_{\mu}, A_{\nu}\right]
$$

where the final term is absent in the Abelian case under discussion. In a supersymmetric theory we might try to construct a feld strength out of the real vector gauge cuperfield, $V$, and covariant (super-)darivatives $D$ and $D$. Under a eauge transformation $F_{\mu \nu} \rightarrow F_{\mu \nu}$ becaute it is constructed from covariant (cauge) derivatives. This new object must be invariant under a supergauge transformation as found in (1.40): $V \rightarrow V+i(\bar{\lambda}-\Delta)$. Writing the field otrength as ô, a differential operator $\hat{O}$ actins on $V$, we demand

$$
\hat{o} v \rightarrow \hat{o} v \text { or } \hat{o}(\overline{\mathbf{a}}-\Delta)=0
$$

under the $U(1)$ rauge transformation where we recall that $\Delta$ and $\bar{\lambda}$ ere ocalar euperfields $(D T=0 ; \overline{D A}=0)$. Chooning $\hat{O}=D$ or $\hat{O}=\bar{D}$ will not work becaue $D L \neq 0$ and $D L \neq 0$ (ond $D L \neq D A$ ). Tryins $\overline{0}=D \bar{D}$ we note that $D \overline{D A}=-\overline{D D} \bar{\Lambda}+(D, \bar{D}] \bar{A}=(D, D) \bar{X}$. Now

$$
\left(D_{0}, \bar{D}_{j}\right)=-2 i 0_{\mu}^{\mu} \partial_{\mu}
$$

and

$$
\left|\partial_{m}, D_{-}\right|=\left|D_{n}, \bar{D}_{j}\right|=0
$$

Therefore $D \overline{D S}=-2 i{ }^{m} \partial_{\mu} \bar{\Lambda} \neq 0$. Hovever, trying the next ordet

$$
\sigma=D^{2} D
$$

does work

$$
\begin{aligned}
& \hat{O} \Lambda=D^{D} D_{\Lambda}=0 \text { sincs } \bar{D} \Lambda=0 \\
& \overline{O A}=D^{3} \overline{D A}=-D D D \bar{\Lambda}+D(D, \bar{D}) \bar{\Lambda} \\
& =-2 i D \sigma^{m} \partial_{\mu} \pi \text { by (1.93) } \\
& =-2 i \sigma^{\mu} \partial_{\mu} D \bar{I} \text { by (1.01) } \\
& =0 \text {. }
\end{aligned}
$$

In the literature $\partial V$ is called $\boldsymbol{W}_{\alpha}$ which is unfortunate since the euperpotential is also called $w$. Its conjugate, $w_{0}$, also watisfies the above criteria and is a field strencth

$$
W_{\phi}=-\frac{1}{4} D D D_{b} V \quad W_{a}=\frac{1}{4} D D D_{a} V .
$$

We note some immediately obvious propertiea of $W_{a}$ 
1. Since $D$ and $D$ are antl-commuting, $W_{0}$ is an antj-commuting object like fand $W_{\Delta}$ is like $T_{b}$.

2. Since $D^{3}=0, D w_{a}=0$ and $W_{a}$ is a chiral superfield.

3. From part 2 we can write $W_{0}=W_{0}(v, 0)$ (just as (or any chiral field) and $W_{\phi}=W_{b}\left(y^{\dagger}, \bar{J}\right)$. We con then use the explicit forms of $D, D$ and $v$ fin Wess.Zumino gauge, of coursel] to expand $w_{a}$ into its component fields

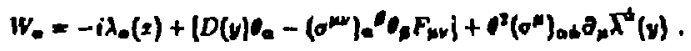

Nole that (on-thell) the final term would vanish by the Dirac equation leaving a gave fermion, auxiliary field $(D)$ and $\sigma^{\mu \nu} F_{\mu \nu}$ term. Since $W_{0}$ is fermion it cannot appeas alone in $\mathcal{L}$ but must appen in a combination wch a $0^{\circ} W_{0}$ of WaW. Also note that the further relation $D^{a} W_{a}=D_{d} W^{+}$is ostisfied. Taking $\mathcal{L}=\int \sigma^{\circ} W_{0} d^{\prime} 0$ would yield (since this project out the $f^{\prime}$ term) a convective o $^{\mu v} F_{\mu \nu}$ term in $\mathcal{C}$ which we don't want is a QED.llke theory. We deaire a term akin to $F_{\mu \nu} F^{\mu \nu}$ so we will try $W^{\circ} W_{Q}=W_{Q} e^{\alpha \beta} W_{b}$

$$
\int W^{\circ} W_{0} d^{2} 0=W^{\circ} W_{0} l_{0}=-2 i \lambda_{0}^{\mu} \partial_{\psi} J-1 F^{\mu \nu} F_{\mu \nu}+D^{\nu}+\frac{i}{4} F^{\mu \nu} \tilde{F}_{\mu \nu} \text { (1.08) }
$$

where $\tilde{F}_{\mu v}=F^{\circ}{ }_{\text {cog }}$. This final term is known to be the source or much pain in anomalous theories. In this paper we will always be concerned with topolcgically trivial solu 8. Then, alter some integration by parts, (ond discarding the, assumed trivial, irface terms) we have the real supersymmetric version of the Lagrangian for a free veclor field

$$
c_{\text {Gave }}=\int W^{a} W_{a} d^{2} Q+\int W_{d} W^{d} d^{2} T=\int D^{s}-\frac{1}{4} F^{w r} F_{\mu L}-i \lambda_{0} \mu \partial_{\mu} T .
$$

(The Fuv $F_{w v}$ is like $E^{2}-B^{2}$ and $F^{\mu v} F_{p v}$ is like $E, B$ ). In the non-Abelian case, just as we had the gauge transformation change from $V \rightarrow V-i(\Lambda-\Lambda)$ to $e^{y} \rightarrow e^{-i \pi_{f} V} e^{i A}$, the superfield strength becomes

$$
W_{*}=-\frac{1}{4} D^{2} e^{-V} D_{0} e^{V}
$$

\section{Construcling Supercymmetric Lazrania}

We now have all of the ingredients necessury for constructing a realistic Laprangian which is supersymmetrically invariant:

(1) The "Kinetic Term" $\int T_{e}{ }^{*}$ pde

This contains kinetic terms for the component feld of $\$$ und the matter-gauge coupling terms doas with all of the correnponding aupersymmetric vertices. In the W-Z gave these terms conserve $R$-parity automatically and all vertices may (qualitutively) be obtuined by replacing fields from atandard vertices in pairs by theit aupersymmetry counlerports, except when this would result in a rew vertex of dironaion greater than four. For exemple, usine (the $\sqrt{2}$ in Wess and Bager notation)

$$
\begin{aligned}
& \phi(y)=\bar{q}(u)+\sqrt{2} 0 \psi(v)+v^{2} F(y) \\
& \boldsymbol{y}^{\prime \prime}=x^{\mu}+\therefore- \\
& \phi(x)=\mid \tilde{\varphi}(x)+i 0 \sigma^{\mu} \tilde{\partial} \partial_{\mu} A(x)+\frac{1}{2} 0^{2} \partial^{2}[A(x) \mid \\
& \left.\left.+\sqrt{2} \theta \psi(x)-\frac{i}{\sqrt{2}} \theta^{2} \partial_{\mu} \psi(x) \sigma^{2}\right\rangle\right]+\partial^{2} F(x) \\
& V=-\theta \sigma^{2} A_{*}-i i^{2} \theta \lambda+h, c .+10^{2} J^{2} D \\
& e^{o v}=1+o v+l v^{2} v^{2}
\end{aligned}
$$


yields (rememberine that $d^{4}$ project the $0^{2} t^{2}$ term out of the expression)

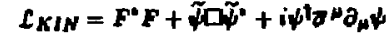

$$
\begin{aligned}
& +\frac{g}{2}\left(\psi^{\dagger} \nabla^{\mu} A_{\mu} \psi+i \tilde{\psi}^{\omega} A_{\mu} a^{\mu} \tilde{\psi}-i \tilde{\psi} A_{\mu} \theta^{\mu} \tilde{\psi}-i \sqrt{2}\left(\bar{\psi} \lambda^{\prime} \psi^{l}-\tilde{\psi} \cdot \lambda \psi \mid\right)\right. \\
& +\frac{1}{\psi} \tilde{\psi} \tilde{\psi}\left(\varrho D-\frac{1}{\xi} a^{2} A_{\mu} A^{\mu}\right)
\end{aligned}
$$

where this is for a simple wealar matter uperfield and a wingle Abelian vector euperfeld. This contains the $\frac{i f}{\sqrt{2}} \tilde{\psi}^{\bullet} \psi \lambda$ "electron-selectson-photino" type of term, the $\frac{i q}{2} \tilde{\psi} * \tilde{\psi}$ derivative scalar electrodynamice term (for velections) and the thal term, which is " "encull" term for the celections. There is no order Gve $\psi^{*} A_{\mu} A^{\mu} \psi$ term untens it is buried in $(g / 2) \tilde{\psi} \cdot \tilde{\psi} D$. Normally the only other term with a " $D$ " in it will be $\frac{1}{D^{2}}$ coming from the feld-strength term below. Thus, wolving for the auxiliary fald $D$ from its equation of motion, will had to a $|\tilde{\psi} \cdot \tilde{\psi}|^{2}$ ursm (i.c. a four-selection vertex) whow coefficient is proportional to $p^{2}$. (In general

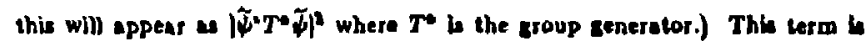
instrumental in initiating spontencous supersymmetry breaking (SSB) (cee point four below). Note that if multiple fields are involved in $f$ that mixing term will naturally result.

(2) The Superpolential term or "F-uector"

$$
\int W|\phi| d^{2} \mid+h_{. c .}=\int\left(\lambda_{i} \phi_{i}+m_{i j} \phi_{i} \phi_{j}+g_{i j} \phi_{i} \phi_{j} \phi_{L}\right) \delta^{2} t+k . c .
$$

This term encompasses the mass terms, Yukawa couplines and potential energy lerm for the matter 6elds," plus the various superaymmetric veriations.

11 There arie in part from FF" in the "Yientic Term" but not enti) a superpolential io added.
R-parity must be imposed. The Higes structure of a theery is lound in this eector. Recall from (1.67) and (1.69) that the individual terma are

$$
\begin{aligned}
& \int \lambda_{i} \phi_{i} t^{*} t=\lambda_{1} F_{i}(v) \text { (often wppreased by } R \text {-parity) } \\
& \int m_{i j} \phi_{i j} \phi^{2} v=2 m_{i j} \tilde{\psi}_{i} F_{j}-m_{i j}(-) \psi_{i} \psi_{j}
\end{aligned}
$$

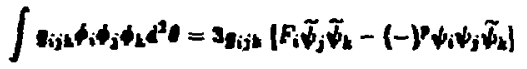

whore the $(-)$ in to remind $w$ that $\psi$ is anti-commuting and so $\psi_{1} \psi_{2}$ and $\psi_{2} \psi_{1}$ enter with opposite sians (positive for $\phi_{1} \psi_{1}$ ) and wo have assumed that $m_{i j}$ and

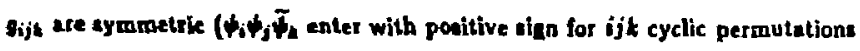
of 123)

We found that this could be written as

$$
\begin{aligned}
& C=W^{\prime} F+W^{\prime \prime} \psi \psi(\text { evaluated at } \phi=\tilde{\psi})+\text { h.c. }
\end{aligned}
$$

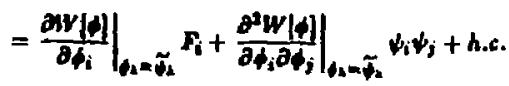

and when the hinetic term was added yielda

$L=$ Kinetic terms + eauge-matter couplinge $-\left|f m_{i j} \psi_{i} \psi_{j}+\theta_{i j k} \psi_{i} \psi_{j} \tilde{\psi}_{k}+h . c.\right|$

$$
-v_{\text {P.C. }}\left(\tilde{\psi} . \tilde{\psi}^{*}\right)
$$

where $V=F+F$ in a potential enercy term and, since the equation of motion for $F$ is $F=-W \cdot, v=|W|^{2}$.

(3) The Super Field Strength term or "D-tector" If $W^{a} W_{c} d^{2} b+h . c$. 
This term includes the free gauge field sector and tis superaymmetric counlerpark. We find the vector and saugino kinetic terms and the amalozue of the Geld strength $F_{\mu \nu} F_{*}^{\mu \nu}$ which, in the non-Abelian ease, includes all of the trilin. ear and quadrilinear gauge self-interactions (and govgino in the trilinear case). Finally we have an auxiliary $D_{i}$ field for exch vertor euper-field $V_{i}$ present

$$
C=-i \lambda_{0} \sigma^{\mu} \partial_{\mu} \lambda_{i}-\frac{1}{4} F_{\mu \nu}^{i} F_{i}^{\mu \nu}+\$ D_{i}^{2}
$$

\section{(4) Fayet-Jliopoulos Prozenitor $\int \mathrm{V}+\mathrm{H}$}

The only term which has not jet been diecussed to function of the vector cuperfields, $V_{i}$ just as $|W| \phi_{i} \mid$ is a function of the scalat auperfields (there is also - groviky superfield but we will not concern ourcelvet with il in this paper).

$W_{5}$ know that, In the $W-z$ zauge, the term $V$ and $V^{2}$ arlae. The term $m \int v^{\prime} d^{4} e$ would introduce vector and sovtino mass terms. Just as such terms are not gauge invariant in ordinary gauge theories, they are not supergaugeinvarient here. Under (the Abelian case) $v \rightarrow v-i(\pi-\Delta)$ the term $\int v^{2} d{ }^{\prime}$ changes by

$$
\int v^{2} d^{4} t \rightarrow \int v^{2} d^{\prime} t+2 \int(\pi \Lambda) d^{2} t
$$

and may be excluded.

The linear term, $c \int V d^{\prime} \theta$, is gauge invarient, however. The reason is that $\int d^{\prime} l \mathrm{~b}$, like $d^{\prime} / d \theta^{\prime}$, a projection operator for the $\left.a^{\prime}\right]^{2}$ term. Therefore

$$
\begin{aligned}
& \int v(x, 0, \partial) d^{2} \theta d^{2} z \rightarrow \int v(x, 0,0) d^{2} U d^{2} z+i\left[\pi(y, 0)-4(y, 0) \mid d^{2} d d^{2} z\right. \\
& =\int V(x, 0, \overline{7}) d^{2} 0 d^{2} 7 \text {. }
\end{aligned}
$$

So this term cannot be ao easily excluded. If $V$ is not $\& U(1)$ zauge superfield (i.e. mediates a non-Abelian force), $v \rightarrow V_{i j}=T_{i j} v \bullet$, then $V$ carries quantum numbers and $f V d$ would explicilly break the ordinary cauge symmetry and would also be excluded. We are lelt with the sole possible term

$$
\mathcal{C}_{F .3 .}=\int \mathrm{C}_{i} V_{i} \mathrm{~d}^{4}=e_{i} D_{i}
$$

where $V_{i}$ are $U(1)$ veetor superfielas and the $c_{i}$ are constants. The $c_{i} D_{i}$ are called "Fayet-Itiopoulos" terms. Their cale function in tife is to alter the auxiliary $D$ field constraint by a constant and thereby initiale spontaneous aupernymetrybreaking. How this is accomplished will be outlined in the following subsection. Since the theory we will be dealing with will be broken by explicit terme we will assume that no F-I type terms arise. This term (for obvious rewons) is frequently included in, the "D-sector" alone with the ouperbeld atrencth term.

\section{Spontaneous Supersymmetry Breakin:}

I intend to gloss over this very brielly since it is not ubed elsewhere in this paper. Supersymmetry may be spontaneously broken both in the " $F$ "sector and the " $D^{n}$ sector. Supersymmetry may also be dynamically broken (e.c. "supercolorn).

Let us first examine the "F" sector. We recall from (1.13) that

$$
H \sim \sum_{i}\left|Q_{i}\right|^{2} \geq 0
$$

Thus, if the vacuum atate has non-zero energy it muat be true that $Q,|0\rangle \neq 0$ for at leust one supersymmetry cenerator $Q_{i}$. The vacuum state is therefore not ennihilated by sueh a ceneratos and supersy umetry is iponteneousiy broken. For supersymmetry to be unbroken we require $H=0$ in the vacuum. $H$ achieves its 
minimum when the polential energy, $\nu=F_{i} F_{i}=\sum_{i}\left|\frac{d F}{\partial f_{i}}\right|^{2}$, vanishes. Therefore we require $F_{i}=0 ;\left.\frac{w}{b_{i}}\right|_{d_{i}=\tilde{\nu}_{i}}=0 V_{i}$ in the vecuum. That $F_{i}=0$ for unbroken eupersymmetry may be understood from the transformations in (1.37). In the vacuum, since there can be no derivative term, we have $(A \neq \bar{\phi})$

$$
\delta_{1} A=\sqrt{2}(\psi) \quad \delta_{1} \psi=\sqrt{2} \ell\langle F\rangle \quad \delta_{1} F=0
$$

Hence when we vary the scalar field we obtain zero unless $(\phi) \neq 0$. This it would be a disuter aince it would imply that the vacuum was not Lorents invariant! (Actually the vacuum can consist of a single, massless, momentumzero fermion.) Jenoring this possibility we see that if $\boldsymbol{F}_{i}=0$ then the vasiation of all of the component fields under a supercymmetric transformation vanishes.

The other formulation of the condition for unbroken superoymmetry. $\left.\frac{\partial \psi}{\partial \psi_{i}}\right|_{f_{i}=\tilde{w}_{i}}=0 \forall \phi_{i}$ for come set of $\tilde{\psi}_{i}$ values (i.e. the vecuum), meane that eupersymmetry is eponteneously broken if the cet of equations, $\frac{w}{d t_{i}}=0$ hes no consistent colution.

\section{Exinples:}

1) $W|\phi|=\phi^{5}$

$$
\left.\frac{\partial W}{\partial \phi}\right|_{\phi=\tilde{w}}=0 \Rightarrow s \tilde{p}=0 \Rightarrow \tilde{\psi}=0 \text { ic a solution. }
$$

2) $W|\phi|=s \phi^{2}+m \phi^{2}$

$$
\left.\frac{\partial \psi}{\partial \phi}\right|_{\phi-\tilde{\sigma}}=0 \Rightarrow 3 g \tilde{\tilde{\psi}^{2}}+2 m \tilde{\psi}=0 \Rightarrow \tilde{\phi}=0 \text { is a solution. }
$$

3) $W\left(\phi_{1} \phi_{2} \phi_{1}\right)=\lambda_{\phi_{1}}+m_{\phi_{2}} \phi_{2}+g_{1} \phi_{2} \phi_{2}$

$$
\begin{aligned}
& \frac{\partial W}{\partial \phi_{1}}=0 \Rightarrow \lambda+g \phi_{2}^{2}=0 \\
& \frac{\partial W}{\partial \phi_{2}}=0 \Rightarrow m \phi_{2}+2 g \phi_{1} \phi_{2}=0 \\
& \frac{\partial W}{\partial \phi_{3}}=0 \Rightarrow m \phi_{2}=0 .
\end{aligned}
$$

The fist and third equations art cleatly inconsistent when $\lambda$ and $m$ are non-zero and we have spontaneous supersymmetry-breaking. This is called the O'Ruifeurtaigh Polential." Unlike internd eymmetry breaking, in which acalar feld acquires a vacuum axpectation value, spontaneous uupersymmetry-breaking occurs when a constraint (auxilinsy) told acquires a VEV. (Note how the "dangerout" single auperifeld term $\lambda \phi$ is again involved. $W=\lambda \phi$ would break supereymmetry by itself but could violate R-parity.)

In the "D" sector the Foyet-Illopoulos term, $C F I=c \int V d A=c D$, provides the breaking. From (1.102), (I.105) and (1.108) we see that (for one Abelian gauge superfield and ose scalar auper inld), when all of the parts of $f$ are included, the terms wilh " $D$ " in them are

$$
C_{D}=\frac{g}{2} \tilde{\nabla} \tilde{\psi} D+\frac{1}{2} D^{2}+C D
$$


so $\frac{\partial \mathcal{C}}{\partial D}=0=\{0 \tilde{\psi} \cdot \bar{\psi}+c+D$ ol

$$
D=-\left(c+\frac{9}{2} \tilde{\psi} \cdot \tilde{\psi}\right) \quad C_{D}=-1\left(c+\frac{9}{2} \tilde{v} \cdot \tilde{\psi}\right)^{2} .
$$

This is a yotential term; $V_{D}$ is to be added to $V_{F}$, which replaces the $|\phi|^{4}$

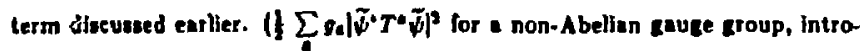
ducins Clebsch-Gordon coefficients into the relative coupling atrengtht.) When $c<0$ the minimum in achieved when $\langle\tilde{\psi}\rangle=\sqrt{\frac{2|c|}{y}}$ and the pauge summelry is spontaneaurly broken. Supersymmetry in unbroken because, in the vacuum, when $\langle\tilde{\psi}\rangle=\sqrt{\frac{2|c|}{y e}}$ wave a vanishins potential and $\boldsymbol{H}=0$ is yet possible (unless broken in the $F$-sector). When $c>0, X_{D}>0$ and we have apontaneous cuperoymmetry-breaking. In thib case the gavge symmetry bunbroken. Note that

$$
\begin{aligned}
& C_{D}=-j D^{2}=-\nu_{D} \\
& \nu_{D}=f D^{3}
\end{aligned}
$$

so we may write the total potential.

$$
v=v_{r}+v_{D}=F_{i}^{u} F_{i}+\mid D_{i} D_{i}
$$

Again we have sponlaneous supersymmetry-breaking when the auxiliary field (D) gets a VEV and gauge breaking when the sealar field does. In general tause and supercymmetrien may be independently conservad or opontaneously broken. In Fis. I.I we summarize this breaking piclure by showing potential ter ms of each of the foup possibilitien (sauge, supersymmetry elther preserved or sponteneously broken). Finally spontaneous superaymmetry-breaking results in a Goldstone Iermion ("Coldstino") jusl as apontaneous gauge rymmetry breaking results in * Goldatone boson.
The epproach actually utilized is simply to add "solt" brenking terms directly into C. Such ter me could stive from spontaneous oupersymmetry-breakins as discussed (at least o priori) at some creater energy scale (opecifically from local wuperty mmetry breakins) but we timply do not worry about such opecific orizins. In point of fact zlobal aponlaneous tuperaymmetty-breaking hes proven singularly unsuccessful in providins realistic models. Supercravlty-inspired noftly-broken theories appear much more promising."

One of the nicest espects of supersymmetry-breaking is that it carnot be induced radiatively (due to improved Sivergence cancellations; there are still non-perturbative instanton-like effects which ean break superoymmetry). There in a "aet it and forget it" attilude. If auperaymmetry la unbroken at the tree level If will remsin 80 to any number of boops. Mases and Yukawa couplings afe not renormalized al all in unbroken superaymmetry (and typically receive only finite correction in spontaneouly or softly broken theories). Only the wavefunctions need be renormalized. The advantage of uring only "soft" breaking terms is that these impressive renormalization properties are nol destroyed.

How does this supersymmetry breaking manifest fielf? Note that both " $D$ " and " $F$ " spontaneous eupersymmetry-breakine give marses to the bosons, but not the fermions, thus lifting the mass degeneracy. The fermions gain mas vis the traditional Higst mechnnism. Ptior to breaking superuymmetry lorces the bosonic superpartners to attain identical mases.

\section{Assembling the Lutrangin: SQED}

We are now ready to put all the ingredients we have discused together to produce a realistic theory. Before going to the supersymmetric alandard model we present the much simplex case of unbroken superoymmetric QED. Since it 
is unbroken we will not require \& Fayet-lliopoulos term (lineur in $V$ ) or a cuperpolential term linear in elther of the scalar supertalds $\phi_{k}$ and $\phi_{R}$. Since there are also no Yukawe terms we may do away with trilinear terms in these oupesfieids. $V$ is the photon auperbeld, $\phi_{L}$ contains $e_{L}$ and its superpertaer $\tilde{e}_{L}$ (the "left-selection") while $\phi_{R}$ contains $\ell_{R}$ and $\tilde{a}_{R}$. The subucripts on $\tilde{\boldsymbol{c}}_{L}$ and $\tilde{e}_{R}$ convey no anguler momentum information (they are ecalars afier allf) but indicate which of the spinor states the boson is ascociated with. Ehewhere $\phi_{L}$ is called $\boldsymbol{L}$ and $\phi_{f}$ is called $\boldsymbol{R}$. This is convenient when we conaider the extention to multiple generations $\left(L_{i}, R_{i}\right)$ and nontrivial (doublat) structure under other groups $(S U(2))$. Note that aince $R$ is a chiral (left-handed) superfiald (a is $L$ ) then $\bar{R}$ must contain $e_{\boldsymbol{R}}^{+}$while $R$ conteins $e_{i}^{+}$. This eccounts for the diferences in charge aigns in the kinetic terms.

Kinetic Term : $\quad L_{e}^{-a V_{L}}+\bar{R} e^{\star v V} R$

Since we desire a Dirac mas, not a Majorane mas, for the electron, we require a term such as $m\left(z_{L} e_{A}+z_{R} e_{L}\right)$ in the Lagrangian.

$$
\text { Superpatential (" } F^{w} \text {-term) : } \quad m_{a}(R L+L F) \text {. }
$$

As usual the (Abelian) superfield strength is given by $W^{a}=-\left\{D^{\top} D^{a} V\right.$.

$$
\text { Gauge Field (" } \left.D^{*}-\operatorname{lerm}\right): \quad \frac{1}{4} W W+\frac{1}{4} W W
$$

Together we have

$$
\begin{aligned}
& \mathcal{L}_{S Q E D}=\int\left\{L_{c}^{-e V} L+K c^{a V} R\right\} d^{4}+\int \frac{1}{4} W^{3} d^{2} 0+\int \frac{1}{4} W^{2} d^{2} \overline{0} \\
& +m_{c}\left\{\int R L d^{*} v+\int I R d^{3}\right\} \text {. }
\end{aligned}
$$

This was presenled in Appendix E (Eq. E3). There we used the fact that $\delta\left(O_{i}\right)=U_{i}$ for any Grassman variable $U_{i}$

$$
\left(\int u_{i} d u_{i}=\int v\left(\theta_{i}\right) d u_{i}=1_{i} \int u_{i} d u_{i}=\int u_{i} \sigma\left(u_{i}\right) d u_{i}=0\right)
$$

to bring the fonal terme Into the form ${ }^{t} t=d e d t \bar{t}$. The expansion into component fields, using the rulas asiduously developed in this chapter, were civen in Eq. (E). 


\section{J.3 THE SUPERSYMMETRIC STANDARD LAGRANGIAN}

We now use the results of the previous sections to construct the supersymmetric version of the "stendard model" (ws of 1987) consisting of 3 generations and an $S U(3) \times S U(2) \times U(1)$ aymmetry Eroup which breaks epontaneously, via the Hisen mechanism, to $s v(3)_{\text {colop }} \times v(1)_{E M}$.

\section{Superfield Content}

Using the notation we have extablished, the superfields requited are given in toble I.1.

The Higes sinclet, $\dot{N}$, may be included to Implement the olandard Hicss mechanism while retainine an unbroken mupercymmetry. Note that $Q, U$ and b are color iriplets while $\dot{G}$ is a color octet.

\section{Kinetic Term}

The general form for this term was found to be

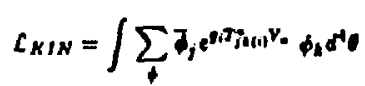

whete "i" sums over all of the couse croups that $\$$ transforms non-trivially under. For Instance, if $\phi=\bar{Q}$. which experiences $s U(3), s U(2)$, and $U(1)$ lorces, then

$$
\left(g_{i} T_{i}^{a} V_{a}\right)_{j k}=g_{\Delta t r o n k} \lambda_{j k}^{c} \dot{G}_{a}+g_{m+n k} r_{j k}^{k} \dot{V}_{a}+g_{j} y \delta_{j k} \dot{V}^{\prime}
$$

Since $f$ is a winglet under $S U(3)$ and $S U(2)$ only the Bnal term would be tequired when $\rightarrow \mathrm{h}$. Here $\lambda^{\circ}$ are the $S U(3)$ generalors.

Wo thall not be concerned with the ection of eluons or sluinos in this paper. Jenorine all but electroweal contributions we can expand $f_{K I N}$ into its component field terms. These consist of atendard kinetic terms for all of the leptons, quarks and Hikgses plus the gauge-coupling terms and their superaymetric counterparts. Letlins ${ }^{17}$

$$
\begin{aligned}
& \forall \text { coupling be }(S U(2)) \quad V^{\prime} \text { coupling be } g^{\prime}\left(U(1)_{y}\right)
\end{aligned}
$$

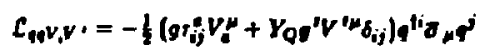

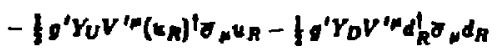
opinor indicea have been suppreased). (1.122) must also be oummed over generelions. Of course we may write $r^{ \pm}=\frac{1}{\sqrt{3}}\left(r^{2} \pm i r^{2}\right)$ and $w^{ \pm}=\frac{1}{\sqrt{2}}\left(v^{3} \pm i v^{2}\right)$ in order to put (1.122) in a more conventional form. For the leptons we have

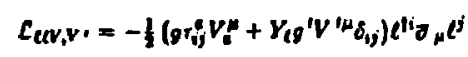

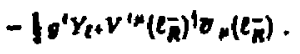

since there is no $\nu_{R}$ term. Other terms which arlse ore (vee (I.102))

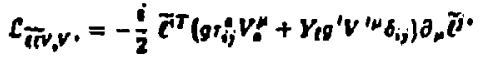

$$
\begin{aligned}
& -\frac{i}{2} v^{\prime} Y_{t}+\tilde{l}_{R} v{ }^{\mu} a_{\mu} \tilde{l}_{R}
\end{aligned}
$$

with a similar expreasion for quasks with $\tilde{C}_{i} \rightarrow \tilde{q}^{i}, \tilde{l}_{R}^{+} \rightarrow \tilde{u}_{R}$ and $\tilde{d}_{R}$. Once again generations are summed over. A related term is

$$
\begin{aligned}
\mathcal{L}_{\bar{\nabla} A}= & \frac{i}{\sqrt{2}} \nabla^{-1}\left(g r_{i j}^{\prime} \lambda_{A} q^{j}+g^{\prime} Y_{Q} \lambda^{\prime} q^{j}\right) \\
& -\frac{i}{\sqrt{2}} \tilde{u}_{R}^{\prime} g^{\prime} Y_{A} \lambda^{\prime} \Psi_{R}-\frac{i}{\sqrt{2}} \tilde{d}_{R}^{\prime} g^{\prime} Y_{D} \lambda^{\prime} d_{R}
\end{aligned}
$$

and similarly for leplont. In addition to these there are ceagull terms, quartic alepton and squark terms and cauge-Hizks terms. For thate terms, re-expressed 
in terms of more femiller form ( $W^{t}$ inutend of $W^{1, x}$, etc.), Refr. 12 and 13 are recormmended. We alrendy cee that the kinetic term alone is anormounly complicated. This 6 only the beginning, however, eince many of these atales mix (apecially when $\left.\left(H_{1,2}^{0}\right),(N) \neq 0\right)$. Furthermore we have auxlliary terme in $L_{K I N}$ which cen only be disentangled once the remeinder of the Lagrenglan is added. Into this we plan to edd cort supersymmetry-branking termal The result is a prodigioualy tangled Lagtangian. For thio reason we will not altempt to simplify ach term as it is presented. We might as well wait until the entire imbroglio hes been accounted for.

\section{Superpotential}

The superpotential term, $W\left(\phi_{i}\right)$, may be divided into the pur Hicre potential and the mas-generation terma (all trilinear)

$W\left[\phi_{i}\right]=\theta_{L}^{i j} H_{1} L_{i} R_{j}+\theta_{D}^{i j} H_{1} Q_{i} D_{j}+\theta_{j}^{i j} H_{2} Q_{i} U_{j}+W_{H}\left[H_{1}, H_{2}, N\right] . \quad(1.126)$

In (j.326) $i$ and $j$ are ceneration indices. The wak boupin indices have been suppressed, however it is assumed that anch term is contracied lato an icosinglet

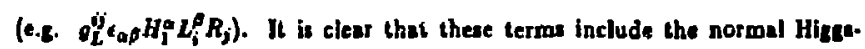
induced lepton and quark mases in cror $=\int W d O+\int W d z$ once the neutral Higga bosons gain a VEV, It is aleo clems that quedrilinens terms euch as $H_{0} H_{0}$ Zl will induce identical masses in these sleptonic partners. As diecussed previously, two Hige doublets are required for such a schems. Note that all of the other inceractions which involve Higesinas will couple with the ene Yukewa olrengths $(g u, g D, g L)$ which will not require radiative renormalizatlons. Note siso that no bilineat potential terms, which would constitute explicit anen terme, have been included.
In order to have unbroken uperaymmetry spontaneo:nly break the gauge symmetry $S U(2)_{W} \times U(1)_{Y} \rightarrow U(1)_{\text {oN }}$ in the Hige uctor it is usual to take

$$
\left.W_{H} \mid \hat{H}_{1}, \hat{H}_{2}, \hat{N}\right\}=g_{N}\left|\hat{H}_{1} \hat{H}_{2}-\right| v^{2} \mid \hat{N}
$$

The equation of motion for the F-term of $N$ is

$$
F_{N}=w_{N}\left(\sigma_{i j} H_{i}^{i} H_{2}^{j}-\frac{1}{j} v^{2}\right)=w_{N}\left(H_{i}^{0} H_{i}^{0}+H_{i}^{-} H_{2}^{+}-\frac{1}{2} v^{2}\right)
$$

where Hi etc. are scalar field components.

Now for auperiymmetry to be unbroken we required (previous section) that $\left(F_{i}\right)=0 v_{i}$. Since $\left\langle H_{1}^{-}\right\rangle_{1}=\left\langle H_{2}^{+}\right\rangle=0$ from charge conservation we require $\left\langle H_{1}^{0}\right\rangle\left\langle H_{2}^{0}\right\rangle \neq 0$. To determine the condition accurately we need to find the potential term, $V=\Sigma_{F} F F^{*}+\ \sum_{D} D^{*}$, from the entite Lagrangian, including the feld-etrength wector. This hes been done in Rer. 12. The above term (1.128) is the only inhomogeneous term in $\nu$ we may aet the VEV of all olber terms to zero. Doins wo the remaining terms are

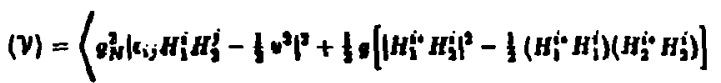

$$
\begin{aligned}
& \left.+\frac{1}{8} g^{\prime 2}\left[H_{i}^{j i} H_{2}^{i}-H_{j}^{j i} H_{i}^{j}\right]^{2}\right\} \\
& =\left.g_{N}^{2}\left|v_{1} v_{2}-\right| v^{2}\right|^{2}+\frac{3}{s^{2}}\left|v_{1}^{2}-w_{2}^{2}\right|^{2}
\end{aligned}
$$

where

$$
v_{1}=\left\langle H_{j}^{0}\right\rangle \quad v_{2}=\left\langle H_{2}^{0}\right\rangle
$$


We see that the solution to $\langle V\rangle=0$ is

$$
v_{1}=v_{1}=\frac{1}{\sqrt{2}} v
$$

We fusther observe that II several singlet superfietds $N_{1} \ldots, N_{K}$ are employed that (I.130) need nol be true. In general it is merely required that

$$
v_{i}^{3}+v_{2}^{2}=v^{2} \quad v_{1}=\left\langle H_{i}^{p}\right\rangle \quad v_{1}=\left\langle H_{1}^{p}\right\rangle
$$

We will, in fact, permit this more general VEV wssignment. We will not include any scalar Hizes superfields since our breaking will be sceomplished via solt explicit terms. The most zeneral Higes strueture with two weak doublets, one singlet and solt (bilinear and certain trilinear) breaking terma has been analyzed. The presence of such soft terous relieves un of the necessity of employing a Hices singlet. (See, in particular, Chapter 3, case 2 of the quoted refarence.) Knowl. edze of the precise detxils underlying the thal Hizes struclure, which will assume the lorta $\sigma_{H}\left|H_{i}^{0} H_{i}^{0 \cdot}+H_{j}^{0} H_{i}^{0 \cdot}-U^{2}\right|^{2}$, need not concern us since they will afrect the Higes sector which we will not be deallng with directly. It is suficient for our purposes to know that such s solt-breaking scheme exists.

\section{Super Field Strength}

Recall that this contribution was of the form

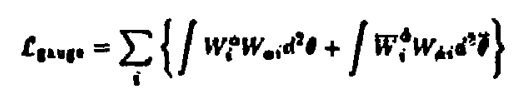

where " $"$ " represents each gauge group in the theory and wo was given by (1.95) and (1.100). Using the notation of Table 1.1 we have

$$
\begin{aligned}
& W_{*}^{\prime \prime}=-\frac{1}{4} \bar{D}^{2} D_{a} \dot{v}^{\prime} \\
& W_{Q}^{\varphi}=-\frac{1}{1} D^{2} e^{-r \cdot \varphi_{0}} D_{0} e^{r \cdot v}
\end{aligned}
$$

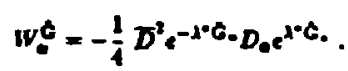

These contain all of the gauge and gaugino hinetic terms and self-interactions. When spontaneous gauge symmetry breaking occurs, zesulting in $S U(2) \times U(1) \rightarrow$ $U(1)_{e m}$, the $\dot{V}$ superfield components becomes (equally) massive. Since caugino mass terms added to $C$ will breat supersymmetry only softly (in fact they are one of the only spin- $f$ fields in an $N=1$ supersymatry tor which this is true) ${ }^{10}$ we will do to. Since this, and the fermion mixing to be diseussed shortly, will ster what we would expet trom (l.133) alone we will gefrain from expandint (I.133) and Into its myrind terors. 
Into the unsullied, fully supersymmetric Lagrangian of the previous section we now add certin terms which break this symmetry soltly.

\section{Soft Breakin: Term:}

"Soft" terms are those, which when included at explicit terms in the Lacrangian at three level, do not introduce new divergences. This is a desirable attribute since it means that the altractive non-senomatization theoreme remain valid. An exhaustive tabulation of soft terme has been made." These include all dimension-1wo operators which would normally arise in $\mathcal{f}$ (once it had been expanded into its component Gelds) and a few dimension three operators. Spacifically included in the latter are geugino mass terme but not sommal feruion masses. Tritinear scalar combinations which ariefe from the unbroken superpotential are also included. Note that vector mass terms would brenk ordinary cauge invariance and are not included. Higesino bilinears of the form $c^{i j} \tilde{H}_{i} \tilde{H}_{j}$ are also acceptable.

The soft terms which we will add to S scu's, the keplonic sectos of "atandard" supersymmet... Lagrangian, are

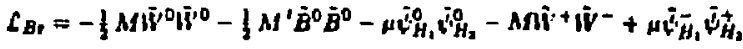

$$
\begin{aligned}
& +m_{i j} \dot{l}_{i} \bar{l}_{j}+\dot{m}_{1,} \dot{p}_{1} \dot{\nu}_{j}+\text { Higes boson terms }+ \text { h.c. }
\end{aligned}
$$

The Higgs boson terms will be responsible for electroweah spontaneous symmetry breaking as discussed previously. There is no $N$ superfield and only $H_{1}^{0}$ and $H_{3}^{0}$ incur non-zero vacuum expectation values

$$
\begin{aligned}
& \left(H_{1}^{0}\right\rangle=v_{1} \quad\left(H_{2}^{0}\right\rangle=v_{2} \\
& v_{1}^{2}+v_{2}^{2}=v^{2}=\left(\frac{2 m_{\omega}}{v}\right)^{2}=(250 \mathrm{GeV})^{2} .
\end{aligned}
$$

It is convenient to define

$$
\tan \theta_{1}=v_{1} / v_{2}
$$

(This is called cot $\rho$ in Rer. 13.)

\section{Slepion Mass Larrangion}

As diccussed is Chopter 3 we may generally ignore the contribution to the ecolat lepton (slepton) mases which erise vis the unbroken superojmmetric Higsa mechanism, from the kpton rawsen, and instead consider the most general mass matrices induced by soft terme:

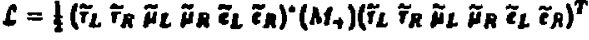

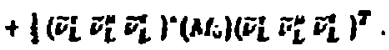

llere we have ignored right-handed sneulrinos. The complexilies of this term in seneral are alluded to in Chaptez 3. For our purposes it is sufficient to simplify the mixing to

$$
\begin{aligned}
& \bar{\mu}_{L} \text { and } \bar{i}_{L} \text { with angle } \boldsymbol{H}_{L} \\
& \hat{\mu}_{R} \text { and } \tilde{r}_{R} \text { with angle } \theta_{R} \\
& i_{\mu} \text { and } i_{i} \text { with angle } i_{\nu}
\end{aligned}
$$


in which ease we may write

$$
\begin{aligned}
& i_{L_{1}}=\tilde{\mu}_{L} \cos \theta_{L}+i_{L} \sin I_{L} \\
& i_{L}=-\tilde{\mu}_{L} \sin i_{L}+i_{L} \cos I_{L} \\
& i_{n_{1}}=i_{R} \cos \theta_{n}+i_{n} \sin \theta_{n} \\
& i_{R_{1}}=-\tilde{\mu}_{R} \sin \theta_{R}+i_{R} \cos \theta_{R} \\
& \bar{\nu}_{L}=\dot{\nu}_{\mu} \cos \theta_{\nu}+\bar{\nu}_{\mu} \sin \phi_{\mu} \\
& \dot{\nu}_{2}=-\bar{\nu}_{\mu} \sin \theta_{\nu}+\dot{D}_{r} \cos \theta_{v} \text {. }
\end{aligned}
$$

Note that $i_{L, n_{1}}, i_{L, n}$, and $\bar{\nu}_{1,2}$ are the physical anes eigenstates. Conserved slobal family number is the only artifact which wauld prevent euch a construction.

The absence of $\tilde{l}_{L}-\tilde{l}_{R}$ woxing be greab simpliseation. The froportance of weh mixing b clear from chapter 2.

\section{Charrinos}

The supersymwetrle partness of the chaszed Higse and rector bosons (hityasinas and gauginas) are collectively referred to as eharginos.

The aupersymmetric counterparts of the $\mathrm{WW}^{+} \mathrm{H}_{\mathrm{j}}^{-\mathrm{H}_{j}^{0}}$, and $\mathrm{W}^{-} \mathrm{H}_{i}^{+} \mathrm{H}$ lerms in the unbroken standard 6 sGW's include

$$
:=-\frac{i g}{\sqrt{2}}\left[H_{1}^{0} \tilde{w}+\bar{\psi}_{H_{0}}+H_{2}^{0} \tilde{w}-\bar{v}_{H_{2}}^{+}\right]+\text {h.e. }
$$

onte $S U(2) \times U(1)$ is broken down to $U(1)_{\text {am }}$ and $\left\langle H_{1,1}^{0}\right\rangle=v_{1,2}$

$$
f=-\frac{i f}{\sqrt{2}}\left[v_{2} \widetilde{W}+\dot{V}_{H_{1}}+v_{2} \widetilde{W}-z_{H_{1}}^{+}\right]+\text {h.e. }
$$

Note that these are Weyl spinors and we have awitehed to the basis $\widetilde{W}^{ \pm}=$ $\frac{1}{\sqrt{2}}\left(\widetilde{W}^{1} \pm i \widetilde{W}\right)$. These terms will induce mixing between the charged Higcsinos and gaveinos. A sirnilar miscibility oecurs in the neutral sector. In this gense the Hikes and gavge superfields have become "united". Fayel ${ }^{30}$ has taken this ldea one atep further. It is known that gauge and Hizes bosone ean be unified in - single superfield in on $\boldsymbol{N}=\mathbf{2}$ supersymmetry. Fayet showed that this is also possible in our $N=1$ supersymmetry if one is prepared to abandon the WessZumino cauge. If a different aet of component helds is eliminated from * vector superfield vis the supergavge mechanism dexcribed in section one, is possible to be left wlih a gauze boson, Weyl gaugiso, a hizer boson and a Weyl Higesino and no suxiliary felds.

To these fermion muss terms are added the soft breaking terms of (1.131) to pield the shargino mass Lograngien

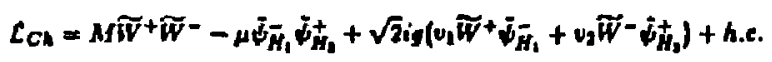

Following Haber and Kane ${ }^{12}$ we note that If we gather thete Weyl delds Into four-component fermions of the forto

$$
\psi^{+}=\left(\begin{array}{c}
-i \widetilde{W}^{+} \\
\dot{\psi}_{H_{2}}^{+}
\end{array}\right) \quad \psi^{-}=\left(\begin{array}{c}
-i \tilde{W}^{-} \\
\dot{\psi}_{H_{1}}
\end{array}\right)
$$

(where we nole that $\vec{\psi} \neq v^{-}$) and write

$$
c_{c h}=-l\left[\left(\psi^{+}\right)^{\tau} x^{\top} \psi^{-}+\left(\psi^{-}\right)^{\tau} x \psi^{+}\right]+\text {h.c. }
$$

(nabe the use of ordinary transposes) then we find that

$$
X=\left(\begin{array}{cc}
M & \sqrt{2} m_{\omega} \cos \theta_{\sigma} \\
\sqrt{2} m \omega \sin \theta & \mu
\end{array}\right)
$$


will reproduce (1.341) to wit

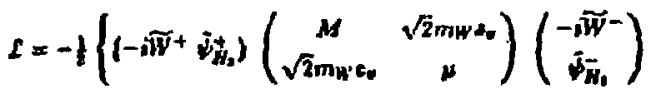

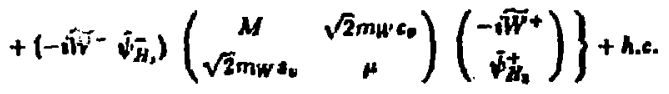

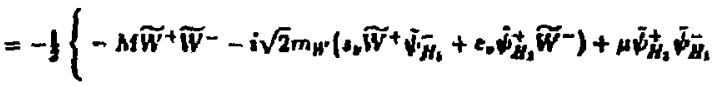

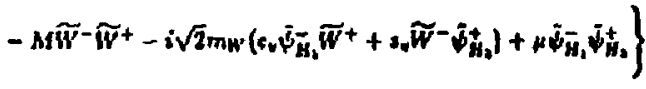$$
+ \text { A.e. }
$$

Now, sitice

$$
\begin{aligned}
& s_{v}=\sin \theta_{v}=\frac{v_{1}}{v} \quad c_{v}=\cos \theta_{v}=\frac{v_{2}}{v} \quad v^{3}=v_{i}^{2}+v_{i}^{*} \quad v=\frac{2 m_{w}}{v} \quad(1.145)
\end{aligned}
$$

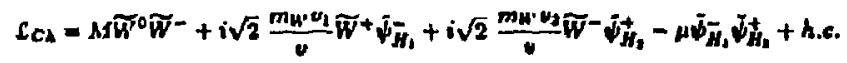

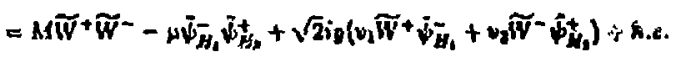

ance we have substituted for " $"$ ". In the nexa appendix we vill diagonalize this to obtain the true chargino mase eigenstates which we desote $\bar{X}_{j}^{+}(i=1,2)$ and $\tilde{x}_{i}^{-}$. When this is diaconalized via

$$
\tilde{x}_{i}^{+}=V_{i j} \psi_{i}^{+} \quad \tilde{x}_{i}^{-}=U_{i j} \psi_{j}^{-}
$$

we will have

$$
L_{C h}=-\frac{1}{2}\left[m_{\tilde{x}_{1}^{+}} \bar{x}_{1}^{-} \bar{x}_{1}^{+}+m_{\dot{x}_{2}^{+}} \bar{x}_{2}^{-} \tilde{x}_{2}^{+}\right]+\text {h.c. }
$$

The malrices $U$ and $V$ (and combinations thereof) will be proliferated through the Feymmen rules. It is also possible lo express $\bar{\chi}^{ \pm}$In terms of the Direc spinors

$$
\widetilde{W}=\left(\begin{array}{c}
-\tilde{W} \tilde{W}^{+} \\
\vec{W}
\end{array}\right) \quad \tilde{H}^{+}=\left(\begin{array}{c}
\tilde{\psi}_{H}^{+} \\
\tilde{H}_{H_{1}}^{-}
\end{array}\right) \text {. }
$$

\section{Nevitritina}

This situntion is similar here but we have more statea. Both Higgs doublets

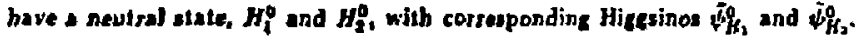
The two neutral auginos are $\tilde{B}^{\circ}$ and $\tilde{W} \circ$, relaled lo the bwis $\tilde{\gamma}$ and $\tilde{Z}^{\circ}$ by the enme sation as their bosonic counterpente

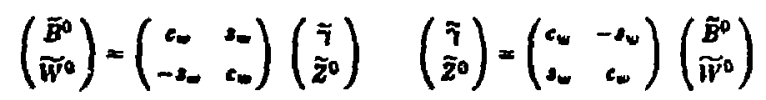

where we henceforth extablish that

$$
\begin{aligned}
& c_{w}=\cos \theta_{0} \quad t_{w}=\sin \theta_{0} \\
& c_{v}=\cos \theta_{0} \quad s_{v}=\sin \theta_{6} \\
& \cos =\cos 20_{0} \quad \sin =\sin 2 \omega_{0} \\
& c_{\phi}=\cos \phi \quad \omega_{\phi}=\sin \phi .
\end{aligned}
$$

From this point forth we ure the traditional natation

$\boldsymbol{\tau} \equiv \boldsymbol{\lambda}$.

The terms derived from the supersyrnmetrized $w^{\circ} H_{i}^{0} H_{j}^{0}$ and $B^{0} H_{1}^{0} H_{j}^{0}$ vertices yikids the mass terms

$$
\frac{i}{2}\left(g^{\prime} \bar{B}^{o}-g \tilde{W}^{\circ}\right)\left(v_{1} \dot{b}_{H_{1}}-v_{2} \vec{u}_{H_{2}}\right)+\text { h.c. }
$$

which again mixts the neutral Hicesinos and gauge bosons. To this we add the 
breakins terms

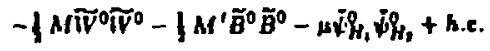

to yield the general "neutralino" Lagrangian

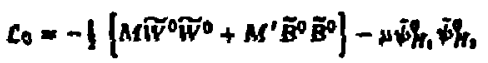

$$
\begin{aligned}
& +\left\{\left(\sigma^{\prime} \tilde{B}^{0}-s \tilde{W}^{0}\right)\left(v_{1} \tilde{v}_{W_{1}}-v_{3} \tilde{w}_{3}\right)+\right.\text { h.e. }
\end{aligned}
$$

In terms of $\bar{z}^{\circ}$ and $\lambda$ this is

$$
\begin{aligned}
& C_{0}=\int\left(M^{\prime} \cos ^{2} \theta_{0}+M \sin ^{2} \theta_{0}\right) \lambda A+\left(M-M^{\prime}\right) \sin \theta_{-} \cos \theta_{0} \lambda \bar{z}^{\circ}
\end{aligned}
$$

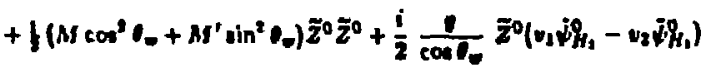

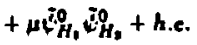

Haber and Kane ${ }^{12}$ establish the notation

$$
\begin{aligned}
C_{0} & =-\psi^{0 \mathrm{~T}} Y \phi^{0} \\
& =-\hbar \psi^{0^{\prime} \boldsymbol{T}} Y^{\prime} \psi^{0^{\circ}}
\end{aligned}
$$

where two possible bases we work with are

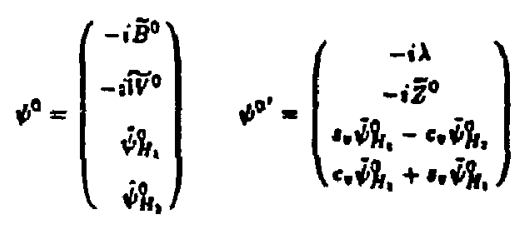

These consist of fout Weyl spinors each. Explicit ealculation thows that

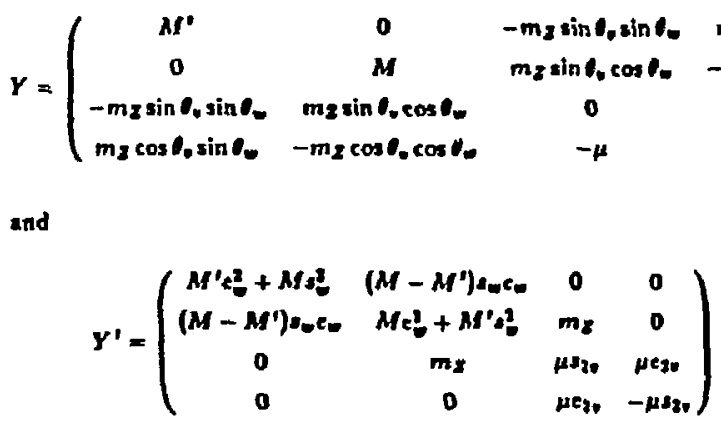

These are related vis

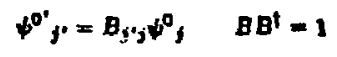

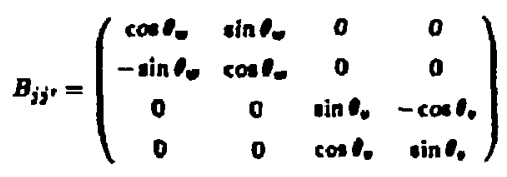

The physical mass eigenstates will be

$$
\begin{aligned}
& \widetilde{x}_{i}^{0}=N_{i j} \psi_{i}^{0} \\
& \tilde{x}_{i}^{\prime}=N_{i j}^{\prime} \psi_{j}^{0}
\end{aligned}
$$

where

$$
N_{i j}=N_{i k}^{\prime} B_{4 j}
$$


now in the form of four-component Majarene epinors

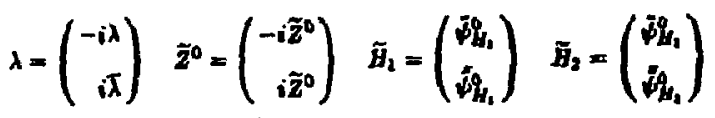

and the eigenstutes as

$$
\tilde{x}_{i}^{0}=\left(\begin{array}{l}
\tilde{x}_{i}^{0} \\
\ddot{x}_{i}^{0}
\end{array}\right) \quad i=1,2,3,4
$$

Gauge Coupling e to Leptons

These are the same as in the standard electroweak Lagrangian (at least at the tree level) and will not be discussed in detail. (Reference 12 expresses this part of the Lagrangian in notation consistent with the superzymmetric terms.)

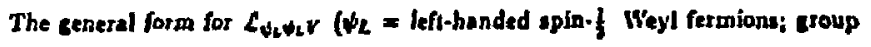
generators are $T^{\text {a }}$ ) is

$$
C_{\psi \psi \psi}=-\operatorname{low} T_{i j}^{\epsilon} V_{\mu}^{*} \psi_{2}^{i} \sigma^{*} \psi \psi_{L}^{c}
$$

In our case (for one generation)

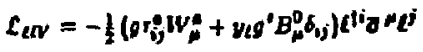

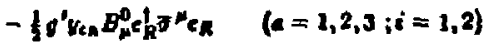

where $\ell^{T}=\left(\ell_{L} v_{L}\right), \tau^{\circ}$ are Pauli matrices and $y$ is byperchare $\left(y t=-1 ; y_{e a}=\right.$ +2). In terms of $A_{\mu}, Z_{\mu}, 1^{\prime *}, \tan \theta_{\omega}=g^{\prime} / g$ and $e=g \sin \theta_{\omega}=\rho^{\prime} \cos \theta_{\omega}$

$$
\begin{aligned}
& c_{\text {lLd }}=-\frac{g}{\sqrt{2}}\left[W_{\mu}^{+} \nu_{\partial} \mu_{L}^{-}+W_{\mu}^{-} C^{+} \partial^{\mu} \mu\right] \\
& C_{2+L^{+} \gamma}=+e C^{+} \gamma^{\mu} A_{\mu} C^{-}
\end{aligned}
$$

213 and $z 0$ forth. In terms of more conventional Dirac apinors, with

$$
\begin{aligned}
& 7 t=f(1 \pm \pi)
\end{aligned}
$$

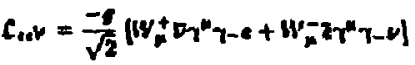

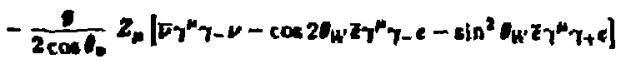

$$
\begin{aligned}
& +\mathrm{CA}_{\mu} \mathrm{ZT}^{*} \mathrm{c} \text {. }
\end{aligned}
$$

Gere Coupling e to Sleptons

Fallowing the procedure used when we derived the SQED lagrangian we find that the analogue of (1.164) 4

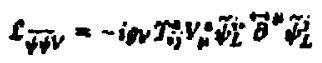

from which $C_{\text {argo, }}$ etc. follows just as in the previous section.

Gavrino Coupling to Lepton:

The corresponding geneal term for $\mathcal{L}_{\text {jor }}$ is

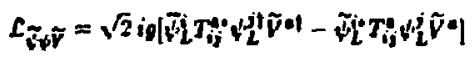

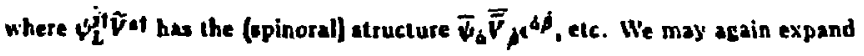
this out tor $\tilde{F}^{*}$ and $\tilde{B}^{\circ}$ and then switch 10 the $\tilde{H}^{\star}, \tilde{z}^{0}, \lambda$ basis. For instance (in tho-component form)

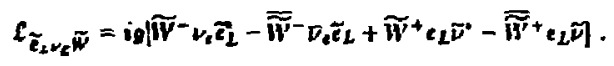

To write this in terms of chatgino mass eigenstates we would have to use (1.142) and (1.146) (or the equivalent expression for (1.146) in terms of (1.148)) and

214 
obtain $\mathcal{C}_{\tilde{C}_{t}} \tilde{x}$, which would have $U$ and $V$ malrices in the coeficients. An example would be

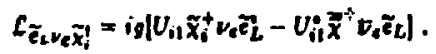

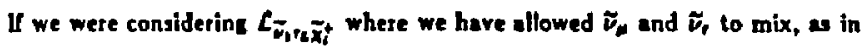
(I.139), then we would bind

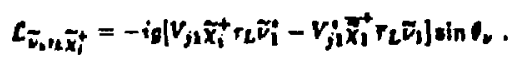

There are obviously many terms and it would be exceedingly tedious to the them all. Wo will restrict our attention to deriving Feynman rules for those vertices which will be of epecial Importance to the ealculations in this work (Appendix K). The same in true of the neutralino vertice.

\section{I.s Gavgino Couplinc to Gauge Bosons}

These are the andogues of the non-Abelian trilinear auge couplings. Once the Hicesinos and Gauginos mix to the physical eigenstates $\bar{x}_{i}$ the vertices $\tilde{x}_{i} \tilde{x}_{j} G_{k}$ and $\tilde{x}_{i} \tilde{x}_{j} H_{k}$ will involve two mixing matrices.

Just as the $G, G_{j} G_{k}$ gauge term is

$$
C_{G G G}=i g r^{i \dagger} G_{i}^{\mu} G_{j}^{\mu} \partial_{\mu} G_{\omega}
$$

the $\bar{G}_{i} \tilde{G}_{j} G_{h}$ term simplities to (Weyl notation)

$$
\mathcal{C}_{\bar{C} \tilde{C} G}=i_{i} j^{i j l} \bar{G}_{i}{ }^{\mu} \bar{G}_{j} G_{\mathrm{hp}}
$$

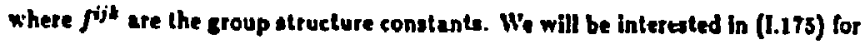
the casea $G=\gamma, z^{0}$.

We will also need the Hicssino-Gauge-Gaugino term. The form of the $H H G$, $H G G$ and $H H G G$ terms axises in the usual manner from GGG and GCGG once we implement spontaneous $S U(2) \times U(1)$ breaking. Since $H_{1}$ and $\hat{H}_{2}$ are acalar euperfields the Lagrangian for $\tilde{G} \tilde{H} H$ and $\sigma \tilde{H} \tilde{H}$ arises in the kinetic terms in the

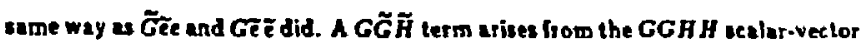
Interaction from the kinetic terw when one of the Hices gains a VEV.

\section{Chargine Coupling to $2^{\circ}$ and 7 i: Weak Eizenbasis}

We will use the notation of Haber and Kane'2

$$
c_{z-\bar{x}_{i}^{+} \tilde{x}_{j}^{-}} \equiv \frac{\theta}{\cos \partial_{w}} z_{m}\left[\frac{1}{2} \bar{x}_{i}^{+} \gamma^{\mu}\left(o_{i j}^{z} \gamma_{-}+O_{j j}^{\prime \mu} \gamma_{+}\right) \bar{x}_{j}^{+}\right]+\text {h.c. }
$$

to define the mixing matrices $O_{i j}^{\prime}$ from the matrices $U$ and $V$ datined in (I.146). We will use the t-component spinors $\tilde{W}$ and $\bar{F}^{+}$in (t.148)and the Majorana spinors from (1.8) and (1.9). 


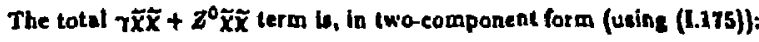

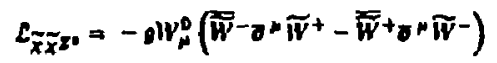

$$
\begin{aligned}
& -\frac{1}{2}\left(\sigma o s_{i j}^{2} w_{\mu}^{0}+g^{\prime} y_{k} \delta_{i j} B_{\mu}^{0}\right)\left(\tilde{\psi}_{H_{b}}\right)^{i} \sigma^{*}\left(\tilde{\psi}_{H_{h}}\right)^{j}+\text { h.c. }
\end{aligned}
$$

(I.177)

where $y_{k}= \pm 1$ can be read of of Table 1.3. The $y_{s}=\left(\begin{array}{cc}1 & 0 \\ 0 & -1\end{array}\right)$ terms include both churgino and neutralino perts.

Using (A.1) and (A.3) we may essemble thin Into Majorane spinors

$$
\begin{aligned}
& \mathcal{L}=-g W_{\mu}^{0}(\bar{W}+) \gamma^{\mu}{\widetilde{W^{\prime}}}^{+}-\frac{1}{2}\left(g W_{\mu}^{0}+g^{0} E_{\mu}^{0}\right)(\bar{H}+) \gamma^{\mu} \tilde{H}^{+} \\
& +\frac{1}{1}\left(g \tilde{H}_{\mu}^{0}-g^{0} \tilde{B}_{\mu}^{0}\right)\left(\tilde{H}_{1} \gamma^{\mu}{ }_{1} \tilde{H}_{1}-\tilde{H}_{2} \gamma^{\mu} \gamma \tilde{H}_{2}\right)+\text { h.c. }
\end{aligned}
$$

The frat two terme constitute the chargino contribution. Using

$$
\begin{aligned}
& z_{\mu}=\cos \theta_{w} W_{\mu}^{0}-\sin \dot{\theta}_{w} B_{\mu}^{0} \\
& A_{\mu}=\sin \theta_{w} W_{\mu}^{0}+\cos \theta_{w} B_{\mu}^{0}
\end{aligned}
$$

results in the chargino part:

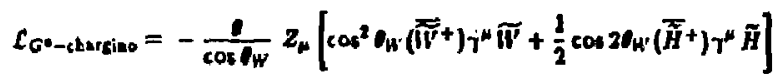

$$
\begin{aligned}
& -e A_{\mu}\left\{(\tilde{\bar{W}}+) \gamma^{\mu} \widetilde{W}+(\overline{\bar{W}}+) \gamma^{\mu} \tilde{H}\right\}+\text { h.c. }
\end{aligned}
$$

\section{Relating Buses}

To texirite this in terms of the ghysical btates $\tilde{x}_{i}^{+}$we need to relate the bases of slates discussed. In cummary:

$$
\begin{aligned}
& \psi_{j}^{+}=\left(\begin{array}{c}
-\tilde{W}^{+} \\
\tilde{\psi}_{w}^{+}
\end{array}\right) \quad \psi_{j}^{-}=\left(\begin{array}{c}
-\widetilde{W}^{-} \\
\tilde{\psi}_{\psi_{1}}
\end{array}\right) \text { from (1.142) } \\
& \tilde{W}_{j}^{+}=\left(\begin{array}{c}
-i \tilde{W}^{+} \\
i\left(\tilde{W}_{-}^{-}\right)
\end{array}\right) \quad \tilde{H}_{j}^{+}=\left(\begin{array}{c}
\tilde{W}_{H_{2}}^{+} \\
\left(\tilde{W}_{H_{1}}^{-}\right)
\end{array}\right) \text {from (1.148) } \\
& \tilde{x}_{1 j}^{+}=\left(\begin{array}{c}
\tilde{x}_{1}^{+} \\
\tilde{x}_{2}^{-}
\end{array}\right) \quad \tilde{x}_{1 j}^{+}=\left(\begin{array}{c}
\tilde{x}_{2}^{+} \\
\tilde{x}_{2}^{-}
\end{array}\right) \quad \text { from (I.148) }
\end{aligned}
$$

where $j=1,2$. Rexall that

$$
\bar{x}_{i}^{+}=V_{i j} \psi_{j}^{+} \quad \tilde{x}_{i}^{-}=U_{i j} V_{j}^{-}
$$

$\left(i_{r} j=1,2\right) \leq 0$

$$
\psi_{j}^{+}=\left(v^{t}\right)_{j i} \tilde{x}_{i}^{+} \quad \psi_{j}^{-}=\left(v^{\dagger}\right)_{j i} \tilde{x}_{i}^{-}
$$

$$
\text { Now } \begin{aligned}
\gamma_{t} & =\xi(1 \pm x) \\
\tau_{t} & =\left(\begin{array}{cc}
-1 & 0 \\
0 & 1
\end{array}\right) \quad \tau_{-}=\left(\begin{array}{ll}
1 & 0 \\
0 & 0
\end{array}\right) \quad \gamma_{+}=\left(\begin{array}{ll}
0 & 0 \\
0 & 1
\end{array}\right)
\end{aligned}
$$

Hence

$$
\begin{aligned}
& \gamma-\widetilde{W}^{+}=\left(\begin{array}{c}
-i \tilde{V}^{+} \\
0
\end{array}\right) \quad \gamma_{+} \widetilde{W}^{+}=\left(\begin{array}{c}
\frac{0}{i \widetilde{W}^{-}}
\end{array}\right) \\
& \gamma_{-} \tilde{H}^{+}=\left(\begin{array}{c}
\tilde{\omega}_{H_{3}} \\
0
\end{array}\right) \quad \gamma_{+} \tilde{H}^{+}=\left(\begin{array}{c}
0 \\
\overline{\tilde{t}_{H}} \\
\tilde{H}_{4}
\end{array}\right) \\
& \tau-\tilde{x}_{i}^{+}=\left(\begin{array}{c}
\tilde{x}_{i}^{+} \\
0
\end{array}\right) \quad \boldsymbol{T}+\bar{x}_{i}^{+}=\left(\begin{array}{c}
0 \\
{\overline{x_{i}}}_{i}^{-}
\end{array}\right)
\end{aligned}
$$

Now, in anticipation of future netds we introduce the conjugate spinors $\widetilde{w}^{2}$ 
and $\bar{x}_{i}^{e}$ (ree also kections A.t and M.t). In general if

$$
v=\left(\begin{array}{c}
t_{*} \\
n^{b}
\end{array}\right)
$$

then

$$
\nabla=\left(\begin{array}{lll}
\eta^{\circ} & \xi_{t}
\end{array}\right) \quad \phi^{\prime}=\left(\begin{array}{l}
\eta_{+} \\
\xi^{+}
\end{array}\right)
$$

10

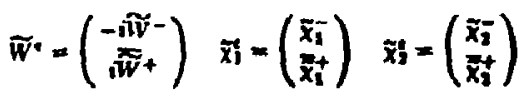

and

$$
\tau_{-} \tilde{W}^{\cdot}=\left(\begin{array}{c}
-i \tilde{W}^{-} \\
0
\end{array}\right) \quad \gamma_{+} \tilde{W}^{*}=\left(\begin{array}{c}
0 \\
i \bar{P}^{+}
\end{array}\right)
$$

(1.187)

From (1.282) and (1.184)

$\gamma-\widetilde{W}_{j}^{+}(1-$ comp. $)=-i \widetilde{w}^{+}(2-\operatorname{comp})=v_{i}^{+}=\left(V^{\dagger}\right)_{12} \tilde{x}_{1}^{+}+\left(V^{t}\right)_{12} \tilde{x}_{2}^{+}$

$=V_{i} \tilde{x}_{i}^{+}+y_{\mathrm{in}} \tilde{x}_{i}^{+}(2-$ comp $)$

$$
=\gamma-\left(v_{1} \bar{x}_{i}^{+}+v_{i 1}^{*} \bar{x}_{2}^{+}\right)(1-\cos p .)
$$

(1.188)

Using (1.181) again, so

$$
\gamma-\bar{w}^{+}=\gamma-\left(V_{i 1}^{*} \bar{x}_{i}^{+}+V_{i 1}^{i} \bar{x}_{i}^{+}\right)
$$

Similarly, from (1.181) and (1.16?)

$$
\begin{aligned}
& -i \widetilde{W}^{-}=w_{i}=v_{i 1}^{\dagger} \tilde{x}_{i}^{-}+v_{i 2}^{\dagger} \tilde{x}_{i} \\
& =U_{i 1}^{n} \tilde{x}_{1}+U_{z_{1}}^{n} \tilde{x_{1}}
\end{aligned}
$$

thus

$$
\begin{aligned}
i \bar{W}- & =U_{11} \bar{F}_{i 1}^{-}+U_{n 1} \bar{x}_{2}(2-\text { comp. }) \\
& =U_{117+\tilde{x}_{1}^{-}}+U_{217+\tilde{x}_{2}}(1-\text { comp. })
\end{aligned}
$$

snd since

$$
7+\widetilde{W}^{+}(1-\text { comp. })=i \widetilde{W}-(z-\operatorname{comp} .) \text { (rom(1.181) }
$$$$
\tau_{4} \tilde{W}^{+}=\tau_{+}\left(U_{11} \bar{x}_{1}^{+}+U_{21} \tilde{x}_{2}^{+}\right)
$$

For the Hizesinos

$$
\tilde{\psi}_{H}^{+}=\psi_{2}^{+}=\left(v^{\dagger}\right)_{21} \bar{x}_{1}^{+}+\left(v^{\dagger}\right)_{22} \tilde{x}_{3}^{+}
$$

$$
=7-\left(V_{i} \tilde{x}_{i}^{+}+V_{j,} \tilde{x}_{g}^{+}\right)
$$

and

$$
\begin{aligned}
& \gamma_{-} \tilde{B}^{+}=\gamma_{-}\left(V_{i j} \bar{x}_{1}+V_{\dot{z}} \mathbf{x} \bar{X}_{1}\right) \\
& \tilde{w}_{M_{1}}=v_{2}^{-}=\left(u^{\dagger}\right)_{21} \tilde{x}_{3}^{-}+\left(u^{\dagger}\right)_{22} \tilde{x}_{i}
\end{aligned}
$$

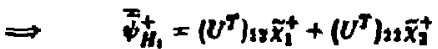

$$
\begin{aligned}
& =\gamma_{+}\left(U_{12} \tilde{x}_{1}^{+}+U_{23} \tilde{x}_{2}^{+}\right)
\end{aligned}
$$

50

$$
{ }_{7+} \tilde{H}^{+}=\gamma_{+}\left(U_{12} \bar{x}_{1}^{+}+U_{22} \tilde{x}_{1}^{+}\right)
$$

as in (1.188)

$$
\begin{aligned}
& -i \widetilde{V}^{+}=\psi_{i}^{+}=\left(v^{+}\right), 1 \tilde{x}_{1}^{+}+\left(V^{+}\right), 12 \bar{x}_{3}^{+} \\
& \Rightarrow \quad \bar{W}^{+}=\left(v^{T}\right)_{11} \bar{x}_{t}^{+}+\left(v^{T}\right)_{2} \bar{x}_{t}^{+}
\end{aligned}
$$

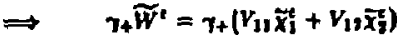


Surmarizing these essulte

$$
\begin{aligned}
& \gamma_{+} \widetilde{W}^{+}=\gamma_{+}\left(U_{1,} \tilde{X}_{3}+U_{21} \tilde{X}_{2}\right) \\
& \gamma-\widetilde{W}^{+}=\gamma-\left(V_{1 i}^{\prime} \tilde{x}_{1}^{+}+V_{3 i}^{+} \tilde{x}_{2}^{+}\right) \\
& \gamma_{+} \tilde{H}^{+}=\gamma_{+}\left(U_{12} \tilde{x}_{1}^{+}+U_{23} \tilde{x}_{2}^{+}\right) \\
& \gamma-\tilde{H}^{+}=\gamma-\left(V_{i 2}^{\prime} \tilde{X}_{1}^{+}+V_{22}^{i} \tilde{X}_{2}^{+}\right) \\
& \gamma_{+} \widetilde{V}^{c}=\gamma_{+}\left(V_{11} \tilde{x}_{i}+V_{21} \bar{x}_{i}\right)
\end{aligned}
$$

(I.100)

Taking the normal Hermilian conjugate in $(1.185)\left(\gamma_{+}=\eta-\right)$

$$
\begin{aligned}
& \widetilde{W^{\prime}} \gamma_{+}=\left(V_{11}{\overline{X_{1}}}_{1}+V_{21} \overline{\bar{X}}_{2}\right) \gamma_{+} \\
& \widetilde{W}_{\gamma-}=\left(v_{11} \bar{x}_{1}+v_{21} \bar{x}_{2}\right)_{-} \\
& \widetilde{H}_{\gamma_{+}}=\left(V_{12} \overline{\bar{X}}_{1}+V_{22} \bar{X}_{2}\right) \gamma_{4} \\
& \overline{\bar{H}} \gamma-=\left(U_{12}^{i} \overline{\bar{x}}_{1}+U_{2 z}^{i} \bar{x}_{2}\right) \gamma-
\end{aligned}
$$

\section{Chargino Coupling to $2^{0}$ and 7 11: Mass Eizenbarie}

We now aubstitute (I.190) and (I.191) into (1.180). We use

$$
\left(\gamma_{ \pm}, \gamma^{\mu}\right)=0 \quad \gamma_{+}+\gamma_{-}=1 \quad \gamma_{ \pm}^{2}=1
$$

(1.192)

to note that

$$
\begin{aligned}
\gamma^{\mu} & =\left(\gamma_{+}+\gamma_{-}\right) \gamma^{\mu}\left(\gamma_{+}+\gamma_{-}\right) \\
& =\gamma_{+} \gamma^{\mu} \gamma_{-}+\gamma_{-} \gamma^{\mu} \gamma_{+} .
\end{aligned}
$$

(1.103)
Then

$$
\begin{aligned}
& L_{z \cdot} \bar{x}^{+} \bar{x}^{-}=\frac{\theta}{\cos \theta_{w}} z_{\mu} \mid-\cos ^{2} a_{w} \bar{W}\left(\gamma-\gamma^{n} \gamma_{+}+\gamma_{+} \gamma^{\mu} \gamma\right) \tilde{W}^{+}
\end{aligned}
$$

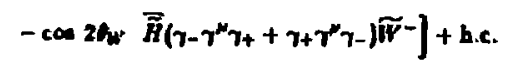

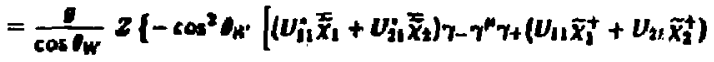

$$
\begin{aligned}
& \text { - } \\
& \left.+\left(V_{11}{\overline{x_{1}}}_{1}+V_{3 j} \widetilde{x}_{2}\right) \gamma_{+} \gamma^{H} \gamma-\left(V_{i} \tilde{x}_{1}^{+}+V_{i 2}^{*} \tilde{x}_{2}^{+}\right)\right] \\
& -\frac{1}{2} \cos 2 \Omega_{W}\left[\left(v_{12}^{+} \bar{x}_{1}^{T}+U_{22}^{*} \bar{x}_{2}\right) \gamma-\gamma^{\mu} \gamma_{+}\left(U_{12} \tilde{x}_{1}^{+}+U_{22} \tilde{x}_{2}^{+}\right)\right. \\
& \left.\left.+\left(V_{12} \overline{\bar{X}}_{1}+V_{22} \bar{x}_{2}\right) \gamma_{+} \gamma^{\mu} \gamma-\left(V_{12} \tilde{x}_{1}^{+}+V_{22} \tilde{x}_{2}^{+}\right)\right]\right\}+ \text {h.c. }
\end{aligned}
$$

Expanding out and collectine terms inlo the form of (1.176) sesults in

$$
\begin{aligned}
& \alpha_{i j}^{L L}=-\cos ^{2} Q_{W} \cdot V_{i 1} V_{j i}^{\prime}-\frac{1}{2} \cos 2 \theta_{H} V_{i 2} V_{j j}
\end{aligned}
$$

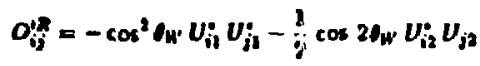

Therefore

$$
\begin{aligned}
& O_{j j}^{\prime 2}=\left(\sin ^{2} \theta_{W^{\prime}}-2\right) v_{i 1} v_{i 1}-\frac{1}{2}\left(1-2 \sin ^{2} O_{W^{\prime}}\right) V_{i 2} v_{j i} \\
& =\sin ^{2} \theta_{13} \cdot\left(V_{i 2} v_{j 1}+v_{12} v_{j 2}\right)-v_{i 1} v_{j 1}-\frac{1}{2} v_{i 2} v_{j 2}
\end{aligned}
$$

The term in brackets is $\left(V^{+}\right)_{i j}=\delta_{i j}$ since $U$ and $V$ are unitary. So finally

$$
\begin{aligned}
& O_{i j}^{\prime L}=\delta_{1}, \sin ^{2} o_{w r}-v_{11} v_{j 1}^{\prime}-\frac{1}{2} v_{i 2} v_{j 2}^{\prime} \\
& \sigma_{i j}^{\prime R}=\delta_{1,} \sin ^{2} a_{w}-U_{i j}^{i} v_{j 1}-\frac{1}{2} U_{i j}^{\prime} v_{j 2} \\
& \mathcal{L}_{z * \tilde{x}_{i}^{-} \tilde{x}_{j}^{-}}=\frac{1}{\cos \theta_{w}} Z_{\mu}\left[\frac{1}{2} \tilde{x}_{i}^{+} \gamma^{\mu}\left(O_{i j}^{L} \gamma_{-}+O_{i j}^{\prime R} \gamma_{+}\right) \tilde{x}_{j}^{+}\right]+\text {h.c. }
\end{aligned}
$$


The photon ter w comes out directly

$$
c_{\bar{x}+\tilde{x}^{-}}=-i e \gamma^{*}
$$

us one might expect.

Neutralino Coupling to $2^{\circ}$ and $z^{x}$

From (I.178) we see that

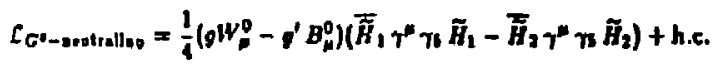

$$
\begin{aligned}
& =\frac{9}{\cos (\omega} z_{\mu} \cdot \frac{1}{4}\left(\bar{H}_{1} \gamma^{\mu}{ }_{n} \tilde{H}_{1}-\bar{H}_{2} \gamma^{\mu} \pi \tilde{H}_{2}\right)+\text { hoe. }
\end{aligned}
$$

where the second line follows from (1.179). Since there to normally no $2^{3}$ of ${ } H^{\circ} H^{0}$ vertex it is not surprising that the only vertex is the supersymmetric analogue of $z H^{0} H^{0}$. The terms the $\tilde{F}_{i} \gamma^{k} \tilde{F}_{i}$ vanish because the neutralinos are Majorana fermions $\left(\vec{x}^{\text {be }}=\vec{x}^{0}\right)$. By analogy with the charging cate wo define

$$
c_{z \circ \tilde{x}_{i} \tilde{x}_{i}}=\frac{1}{\cos \theta_{w}} z_{w}\left[\frac{1}{2} \bar{x}_{i}^{0} \gamma^{\mu}\left(O_{i j}^{n} \gamma_{-}+o_{i j}^{n \pi} \gamma_{+}\right) \bar{x}_{j}^{0}\right]+\text { hic. }
$$

Recalling that $\tilde{X}_{i}^{0}=N_{i j} \psi_{i}^{0}$ we may derive expressions similar to $(1.100)$ and
(1.191):

$$
\begin{aligned}
& \text { 7- } \left.\tilde{B}^{0}=\right\urcorner-N_{j 1}^{0} \tilde{x}_{j}^{0} \\
& { }_{\gamma_{+}} \tilde{B}^{0}=\gamma_{+} N_{j 1} \widetilde{x}_{j}^{0} \\
& 7-\widetilde{W}^{0}=7-N_{j 2}^{0} x_{j}^{0} \\
& { }_{T+} \widetilde{W}^{0}=\tau_{+} N_{j 2} \bar{X}_{j}^{0} \\
& \gamma-\bar{H}_{1}=\tau-N_{j} ; \overline{x_{j}} \\
& \tau_{+} \tilde{H}_{j}=\gamma_{+} N_{j,} \widetilde{X}_{j}^{0} \\
& \gamma-\ddot{H}_{2}=\boldsymbol{\gamma}-N_{j} \tilde{x}_{j}^{0} \\
& \gamma_{+} \tilde{H}_{3}=\gamma_{+} N_{j 1} \vec{x}_{j}
\end{aligned}
$$

where $j$ is summed over $(j=1,2,3,4)$. The remarkable symmetry to due to the fact that $\widetilde{X}_{i}^{0}$ is a Majoratna fermion. Substituting (I.199) into (I.197) and using

$$
\boldsymbol{\gamma}=\boldsymbol{\gamma +}-\boldsymbol{\gamma}
$$

with (1.192) and (1.193), and then placing the result in the form of (J.198) gives:

$$
\begin{aligned}
& O_{i j}^{n L}=-\frac{1}{2}\left(N_{i j} N_{j i}-N_{i 1} N_{j 1}^{*}\right] \\
& O_{i j}^{n n}=\frac{1}{2}\left[N_{i 3}^{*} N_{j 3}-N_{i 1}^{*} N_{j 4}\right]
\end{aligned}
$$

Note that

$$
O_{i j}^{n K}=-O_{i j}^{N L \cdot}
$$

and that photons do not couple to the neutralinos.

225

221. 
Table 1.1. Fields Appearing in the Minimal Model

\section{FIGURE CAPTIONS}

\begin{tabular}{|c|c|c|c|c|}
\hline \multirow[t]{2}{*}{ Superfield } & Ordinary Matter & Superpartners & Weal Laspin & $y$ \\
\hline & \multicolumn{4}{|c|}{ Vector (Goupt) Afultiplets } \\
\hline $\mathbf{E}$ & $\theta^{i}, i=1, \ldots, 0$ & $\theta^{i}, i=1, \ldots, 0$ & Singlet & 0 \\
\hline$\dot{\mathbf{v}}$ & $W \pm, 0$ & $\overline{w^{ \pm}}$ & Triplet & 0 \\
\hline \multirow[t]{2}{*}{$\hat{\mathbf{v}}^{\prime}$} & $B^{0}$ & $\dot{\boldsymbol{B}}^{\mathfrak{a}}$ & Sisiglet & 0 \\
\hline & \multicolumn{4}{|c|}{ Sealar Mfulliplele } \\
\hline $\mathbf{Q}_{i}$ & $u_{L, 1} d_{L}$ & $\tilde{u}_{L_{i}}, \tilde{d}_{L_{i}}$ & Doublet & $1 / 3$ \\
\hline$\dot{\mathbf{u}}_{i}$ & $\left(G_{L}\right)_{L}=\left(w_{R}\right)^{\bullet}$ & $\left(\bar{w}_{R}\right)^{\prime}$ & Singlet & $-1 / 3$ \\
\hline$\dot{\mathbf{D}}_{\mathbf{i}}$ & $\left(d_{1}\right)_{L}=\left(d_{R_{n}}\right)^{\prime}$ & $\left(\bar{d}_{R}\right)^{*}$ & Singlet & $2 / 3$ \\
\hline $\mathbf{L}_{i}$ & $\nu_{i}, \ell_{L_{i}}^{-}$ & $\bar{\nu}_{i}, \tilde{l}_{L}$ & Duublet & -1 \\
\hline $\mathbf{R}_{\mathbf{i}}$ & $\left(t_{n}^{+}\right)_{L}=\left(\ell_{R_{n}}^{-}\right)^{+}$ & $\left(\tilde{t}_{R}\right)^{0}$ & Singlat & 2 \\
\hline$\dot{\mathbf{H}}_{\mathbf{1}}$ & $H_{1}^{0}, H_{1}^{-}$ & $\psi_{H_{1}}, \bar{\psi}_{H_{1}}$ & Doublet & -1 \\
\hline \multirow[t]{2}{*}{$\dot{\mathbf{H}}_{2}$} & $\mathrm{H}_{2}^{+}, \mathrm{H}_{2}^{\circ}$ & $\bar{\nu}_{H,}^{+}, \bar{\nu}_{H, ~}^{0}$ & Doubht & 1 \\
\hline & \multicolumn{4}{|c|}{ Oplional Higes Singlel } \\
\hline$\dot{N}$ & $N$ & $\dot{N}_{L}$ & Singlet & $\mathbf{0}$ \\
\hline
\end{tabular}

1. Palterns of Guuge and Supernymmetry Brenking.

(a) Supersymotry Unbroken and Guze Symmetry Unbroked.

(b) Supersymmetry Broken and Gauge Symmelry Unbroken.

(c) Superaymmetry Unbroken and Gauge Symmetry Broken.

(d) Supersjmmetry Broken and Gauge Symmetry Broken. 


\section{APPENDIX 3}

Mlixing Matrices and Mass Eigenvalues

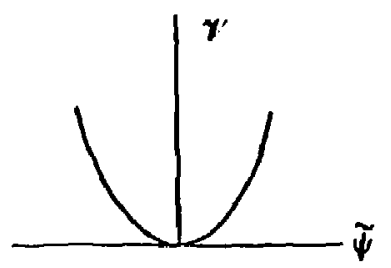

(0)

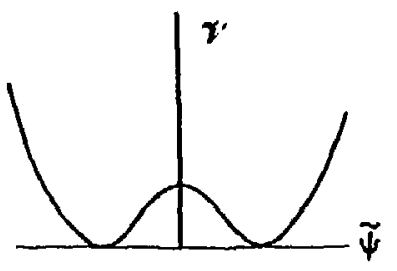

$0.87 \quad(c)$

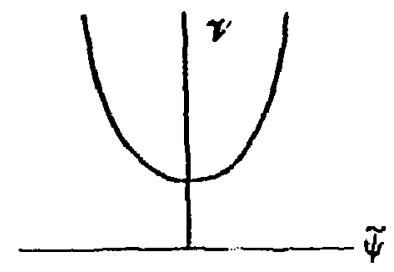

(b)

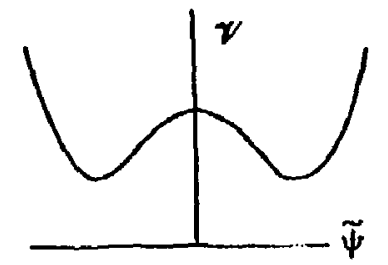

(d) B713A17
Fig. I.I

\section{J.1 Charginos}

As ne saw in the previous appendix (eqns J.142-J.J4B) the charcino mass terms may be written as

$$
c_{c b}=-f\left[\left(\psi^{+}\right)^{T} x^{T} \psi^{-}+\left(\psi^{-}\right)^{\top} X \psi^{+}\right]+\text {h.e. }
$$

where $\psi^{ \pm}$are four-component fermions of the form

$$
\psi^{+}=\left(\begin{array}{c}
-\widetilde{W}^{+} \\
\bar{\psi}_{H_{2}}^{+}
\end{array}\right) \quad \psi^{-}=\left(\begin{array}{c}
-i \tilde{W}^{-} \\
\tilde{\psi}_{\tilde{H}_{1}}
\end{array}\right)
$$

and

$$
X=\left(\begin{array}{cc}
M & \sqrt{2} m_{w} \cos \theta_{\psi} \\
\sqrt{2} m_{w} \sin \theta_{\psi} & \omega
\end{array}\right) .
$$

We may diagonalize this to obtain the true chargino mass eigenstater which we denote $\tilde{x}_{i}^{+}(i=1,2)$ and $\tilde{x}_{i}^{-}$. When this is diaconalized via

$$
\tilde{x}_{i}^{+}=v_{i j} v_{i}^{+} \quad \tilde{x}_{i}^{-}=v_{i j} \psi_{i}^{-}
$$

we will have

$$
\mathcal{L}_{C A}=-1\left[m_{\bar{x}_{1}^{+}} \bar{x}_{1}^{-} \tilde{x}_{1}^{+}+m_{\tilde{x}_{2}^{+}} \tilde{x}_{i}^{-} \tilde{x}_{2}^{+}\right]+\text {h.e. }
$$

The matrices $U$ and $V$ are unitary $2 \times 2$ matrices which diagonalize the woss matrix vis

$$
M_{\text {Dia }}=U \cdot X V^{-1} .
$$




\section{Diazonalization of General 2 × 2 Matrix}

In general to diagonalize a reguler $2 \times 2$ matrix

$$
\left(\begin{array}{ll}
M_{11} & M_{12} \\
M_{21} & M_{22}
\end{array}\right)
$$

by $M_{p}=U \cdot M V^{1}, U$ and $V$ unitary, to

$$
M_{D}=\left(\begin{array}{cc}
M_{+} & 0 \\
0 & M_{-}
\end{array}\right)
$$

we use

$$
\begin{aligned}
& U=\left(\begin{array}{cc}
\cos \phi_{-} & \sin \phi_{-} \\
-\sin \phi_{-} & \cos \phi_{-}
\end{array}\right) \quad V=\left(\begin{array}{cc}
\cos \phi_{+} & \sin \phi_{+} \\
-\sin \phi_{+} & \cos \phi_{+}
\end{array}\right) \quad D>0 \\
& U=\left(\begin{array}{cc}
\cos \phi_{-} & \sin \phi_{-} \\
-i \sin \phi_{-} & i \cos \phi_{-}
\end{array}\right) \quad V=\left(\begin{array}{cc}
\cos \phi_{+} & i \ln \phi_{+} \\
-i \sin \phi_{+} & i \cos \phi_{+}
\end{array}\right) \quad D<0
\end{aligned}
$$

where

$$
D=\operatorname{det} M=M I_{12} M_{23}-M_{12} M_{21}
$$

yielding the positive definite mass eigenvalues

$$
\begin{array}{ll}
M_{ \pm}=\xi\left(M_{+} \pm M_{-}\right) & D>0 \\
M_{ \pm}=\frac{1}{2}\left(M_{-} \pm M_{+}\right) & D<0 .
\end{array}
$$

Here

$$
M_{ \pm}=\sqrt{\left(M M_{11} \mp M_{22}\right)^{2}+\left(M_{12} \pm M M_{21}\right)^{2}}
$$

This is an appropriate time to interject a word concerning the positivity of the eigenmasses. Normally, when diagonalizing a matrix one cusnot be so cavalier about altering the sign of an eigenvalue. When the determinant of a real $2 \times 2$ matrix is megative it indicates one positive and ore negative eigenvalue. It has been noted (and can be easily demonstrated) that negating the eigenmass of a opinor is physically equivalent to sateining the original sign and performing the substitution $\psi \rightarrow \gamma \boldsymbol{b}$ (or equivalently $\psi \rightarrow-\gamma b \psi$ ). Here we have accomplished the same and by multiplying a given row of toth $U$ and $V$ by a factor of $i$. This negates thie corresponding eigenvalue because we have used (J.6) inctead of the purels unitary $\boldsymbol{M}_{D}=\boldsymbol{U} M \mathrm{~V}^{\prime}$. In purticular we note that

$$
\begin{array}{ll}
v v^{t}=v v^{t}=t & D>0 \\
v v^{t}=v v^{t}=-v & D>0 .
\end{array}
$$

We may similarly negate neutralino mases. The angular entries atisfy

$$
\begin{aligned}
\tan \left(\phi_{+} \pm \phi_{-}\right)= & \frac{M_{12} \pm M_{21}}{M_{11} \mp M_{22}} \\
\cos \left(\phi_{+} \pm \phi_{-}\right)= & \frac{M_{11} \mp M_{21}}{M_{ \pm}} \\
\sin \left(\phi_{+} \pm \phi_{-}\right)= & \frac{M_{12} \pm M_{21}}{M_{ \pm}} \\
\tan 2 \phi_{ \pm}= & \frac{B_{ \pm}}{A_{ \pm}} \\
& A_{ \pm}=\left(M_{11}^{2}-M_{22}^{2}\right) \pm\left(M_{21}^{2}-M_{12}^{2}\right) \\
& \left.B_{-}=2 ! M_{11} M_{11} M_{12}+M_{21}+M_{22}\right] \\
\tan \phi_{1}= & \frac{\sqrt{A_{ \pm}^{2}+B_{ \pm 2}^{2}}-A_{ \pm}}{B_{ \pm}} .
\end{aligned}
$$




\section{Charrinos: General Care}

We proceed to ute the above resulta for the general chargino paltern given by (3.3). The mases of the churginos $\left(\bar{x}^{+}\right)$are given in terms of the supersymmetry breaking terms of $M, M^{\prime}$ and $\mu$ of eqn (3.1) and the Hizs, VEV angle, $C_{v}$, of eqn (3.6) (bee section $1.4 \mathrm{~s} / \mathrm{so}$ ) by

$m_{x_{i, 1}}=\mid\left(M_{+} \pm M_{-} \eta_{ \pm} \quad M_{ \pm}=\sqrt{(M \pm \mu)^{2}+2 M_{W}^{2}\left(1 \mp \operatorname{tin} 26_{-}\right)^{2}}\right.$

with $\eta_{+}=1$ and $\eta_{-}=\operatorname{aign}\left[M f_{\mu}-M_{*}^{2} \operatorname{in} 2 \ell_{\nu}\right]$

The masses $m_{x_{i}^{*}}$ have been plotted, for typical parometer values, in Fis. J.Is. The chartino vertex matrices are given by ${ }^{44}$

$$
\begin{aligned}
& U_{i j}=\left(\begin{array}{cc}
\cos \phi_{-} & \sin \phi_{-} \\
-\sin \phi_{-} & \cos \phi_{-}
\end{array}\right) \quad V_{i j}=\left(\begin{array}{cc}
\cos \phi_{+} & \sin \phi_{+} \\
-\sin \phi_{+} & \cos \phi_{+}
\end{array}\right) \quad \Gamma>0 \\
& U_{i j}=\left(\begin{array}{cc}
\cos \phi_{-} & \sin \phi_{-} \\
-i \sin \phi_{-} & i \cos \phi_{-}
\end{array}\right) \quad V_{i j}=\left(\begin{array}{cc}
\cos \phi_{+} & \sin \phi_{+} \\
-i \sin \phi_{+} & i \cos \phi_{+}
\end{array}\right) \quad \mathrm{D}<0
\end{aligned}
$$

where

$$
\begin{aligned}
& D=M \mu-M_{W}^{2} \sin 20, \\
& \tan \phi_{t}=\frac{\sqrt{A_{ \pm}^{2}+B_{ \pm}^{2}}-A_{t}}{B_{t}} \\
& A_{ \pm}=\left(M^{2}-\mu^{2}\right) \mp 2 M M_{M}^{2} \cos 20 \\
& B_{+}=2 \sqrt{2} M_{W}\left(M / \cos \theta_{+}+\mu \sin \theta_{+}\right) \\
& B_{-}=2 \sqrt{2} M M_{W}\left(M P \sin \theta_{U}+\mu \cos \theta_{\theta}\right) \text {. }
\end{aligned}
$$

Recall lrom Appendix $I$ that tbe genetal form of the non-Abelian supersjimmetric vertex was given by

$$
\begin{aligned}
& O_{i j}^{L^{\prime}}=-V_{i 3} V_{j 1}^{\prime}-\mid V_{i 2} V_{j 2}+\delta_{1 j} \sin ^{2} \theta_{0} \\
& o_{i j}^{\prime}=-U_{i 1} \cdot U_{j 1}-j U_{i 2} \cdot U_{j 2}+\delta_{i j} \sin ^{2} U_{w}
\end{aligned}
$$

resulting in the general expressions

$$
\begin{aligned}
& o_{i j}^{L^{\prime}}=\left(\begin{array}{cc}
1 \sin ^{2} \phi_{+}-\cos ^{2} \theta_{\theta} & \mid \sin \phi_{+} \cos \phi_{+} \\
1 \sin \phi_{+} \cos \phi_{+} & \mid \cos ^{2} \phi_{+}-\cos ^{2} \phi_{\omega}
\end{array}\right) \\
& O_{i j}^{n^{\prime}}=\left(\begin{array}{cc}
\frac{1}{\sin ^{2} \phi_{-}-\cos ^{2} \theta_{-}} & \mid \sin \phi_{-} \cos \phi_{-} \\
\mid \sin \phi_{-} \cos \phi_{-} & \mid \cos ^{2} \phi_{-}-\cos ^{2} \theta_{w}
\end{array}\right) \\
& \text { for } D>0 \\
& o_{i j}^{L^{\prime}}=\left(\begin{array}{cc}
1 \sin ^{2} \phi_{+}-\cos ^{2} \theta_{-} & -1 / 2 \sin \phi_{+} \cos \phi_{t} \\
1 / 3 \sin \phi_{+} \cos \phi_{+} & 1 \cos ^{2} \phi_{+}-\cos ^{2} \theta_{-}
\end{array}\right) \\
& O_{i f}^{a^{\prime}}=\left(\begin{array}{cc}
\mid \sin ^{2} \phi_{-}-\cos ^{2} \phi_{0} & 1 / 2 \sin \phi_{-} \cos \phi_{-} \\
-1 / 2 \sin \phi_{-} \cos \phi_{-} & \mid \cos ^{2} \phi_{-}-\cos ^{2} \phi_{-}
\end{array}\right)
\end{aligned}
$$

$$
\text { for } D<0 \text {. }
$$




\section{Charrinos: Speciel Caue}

Here we examine 1 few specific subcuses of interest.

1) $M_{3 g}=M_{3}\left(w_{2}=v_{3}\right)$

When diagor wining the general $2 \times 2$ sjmmetric matrix $\left(M I_{12}=M I_{21}\right)$ we find

$$
\begin{array}{ll}
U=V=\left(\begin{array}{cc}
\cos \phi & \sin \phi \\
-\sin \phi & \cos \phi
\end{array}\right) & D>0 \\
U=V=\left(\begin{array}{cc}
\cos \phi & \sin \phi \\
-i \sin \phi & i \cot \phi
\end{array}\right) & D<0
\end{array}
$$

where

$$
D=\operatorname{deL} M=M_{11} M_{22}-M_{12}
$$

and in this instance

$$
\phi_{+}=\phi_{-}=\phi
$$

which is given by

$$
\begin{aligned}
& \tan \phi=\frac{\sqrt{A^{2}+B^{3}}-A}{B} \\
& A=M_{13}^{2}-M_{32}^{2} \\
& B=2 M_{12}\left(M_{11}+M_{72}\right) .
\end{aligned}
$$

The pasitive eigenvalues are

$$
M_{ \pm}=\left\{\left\{\sqrt{\left(M_{11}-M_{32}\right)^{2}+4 M_{12}^{2}} \pm\left(M_{11}+M_{22}\right)\right\} .\right.
$$

For charginos the symmetric condition implies that $\tan \theta_{0}=1$. Restricting our- selves $t_{0}-\pi \leq \theta_{0} \leq \mathrm{x}$ we conclude that $\boldsymbol{y}_{3}=\mathrm{u}_{2}$. Thea

$$
\tan 4=\frac{\sqrt{A^{0}+B^{b}}-A}{B}
$$

$$
\begin{aligned}
& A=M^{2}-\mu^{2} \\
& B=2 M_{W}(M+\mu)
\end{aligned}
$$

and

$$
M_{ \pm}=\left\{\left\{\sqrt{(M-\mu)^{2}+M M_{N}} \pm(M+\mu)\right\}\right.
$$

are the masse.

2) $M \rightarrow 0, \mu \rightarrow 0 m \neq n$

This corresponds to the instance of the general $2 \times 2$ matrix in which it is completely off-diagonat, i.c. $M_{21}=M_{22}=0$ but $M_{12} \neq M_{21}$. In this case some care to required when using the general expressions. There are two equally valjd solutions:

$$
\begin{aligned}
& \psi_{-}=3, \psi_{+}=\frac{\pi}{2} \text { leading to } M_{D}=\left(\begin{array}{cc}
M I_{12} & 0 \\
0 & M I_{21}
\end{array}\right) \\
& \phi_{-}=\frac{\pi}{2}, \phi_{-}=0 \text { leadine to } M_{D}=\left(\begin{array}{cc}
M_{31} & 0 \\
0 & M_{12}
\end{array}\right)
\end{aligned}
$$

For charginos $M_{12}=\sqrt{2} m_{w} \cos \theta_{4}$ and $M_{21}=\sqrt{2} m_{w} \sin \theta_{4}$. The corresponding 
$U, V$ and $O^{\prime}$ malrices we, for the tirat case,

$$
\begin{aligned}
& U=\left(\begin{array}{ll}
1 & 0 \\
0 & i
\end{array}\right) \quad\left(\begin{array}{cc}
0 & 1 \\
-i & 0
\end{array}\right) \\
& O_{i j}^{L^{\prime}}=\left(\begin{array}{cc}
1-\cos ^{2} \theta_{\omega} & 0 \\
0 & -\cos ^{2} \theta_{-}
\end{array}\right) \quad O_{i j}^{\prime^{\prime}}=\left(\begin{array}{cc}
-\cos ^{2} \theta_{-} & 0 \\
0 & 1-\cos ^{2} \theta_{0}
\end{array}\right)
\end{aligned}
$$

3) "sufr" Limit: $M=\mu=0, v_{2}=v_{2}$

As sbove, the mass matsix is tompletely onti-diagonal howerer, in this case, the on-diagonal terms are equal as well. The solutions $\phi_{ \pm}=0$, $\frac{5}{2}$ apply as before bul now there are additional possibilities. Any $\phi_{-}, \phi_{+}$oubject to $\phi_{-}+\phi_{+}=\frac{\pi}{2}$ is a valid volution. For estetic ressons we will chose to we the oymmetric reault

$$
1-1+=\frac{\pi}{4} \text {. }
$$

The resulting mixing matrices are

$$
\begin{aligned}
u & =v=\frac{1}{\sqrt{2}}\left(\begin{array}{cc}
1 & 1 \\
-i & i
\end{array}\right) \\
O_{i j}^{L^{\prime}} & =\frac{1}{4}\left(\begin{array}{cc}
4 \sin ^{2} \theta_{-3}-3 & -i \\
i & 4 \sin ^{2} \theta_{-3}-3
\end{array}\right) \\
O_{i j}^{\prime} & =\frac{1}{4}\left(\begin{array}{cc}
4 \sin ^{2} \theta_{-}-3 & i \\
-i & 4 \sin ^{2} \theta_{-3}-3
\end{array}\right)
\end{aligned}
$$

4) "Unmixed Limit" $M \rightarrow \infty, \mu=0$

Assuming that we set $\mu=0$ prior to taking the limit $M \rightarrow \infty$, we see that $D=\operatorname{det} X$ is negative. We find

$$
\begin{aligned}
& M_{+}=M \rightarrow \infty \\
& M_{-} \rightarrow \tau \text { as } M_{W}^{2} / M
\end{aligned}
$$

etgo we have one very massive and one massleas pasticle. We also delermine that

$$
\tan \phi_{ \pm} \rightarrow 0 \approx M w / M
$$

co that

$$
\begin{aligned}
& U=V=\left(\begin{array}{ll}
1 & 0 \\
0 & i
\end{array}\right) \\
& O_{i j}^{L^{\prime}}=O_{j j}^{R^{\prime}}=\left(\begin{array}{cc}
-\cos ^{2} & 0 \\
0 & -\cos ^{2} \theta_{0}
\end{array}\right)
\end{aligned}
$$

\section{J.2 NeUtralinos}

From $\mathcal{L}_{\mathrm{B}}+\mathcal{L}_{M i 2} \mathrm{i}$ eqni $(3.4)$ end (3.6) we can wrlle the neutralino mass terms in the form: ${ }^{12}$

$$
\mathcal{L}_{\text {mase }}=-f\left(\psi^{0}\right)^{7} y \psi^{0}+\text { h.e. }
$$

where $Y$ was given by eqn (3.8) ot eqn (I.156) and

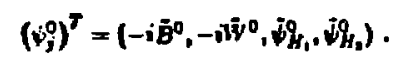

$Y$ is diazonalized by the unitary matrix $N$ vis $M_{D}=N^{\circ} Y N^{\dagger}$ (we can ehoose $N^{*}$ innlead of $N$ because we use $\left(\psi^{0}\right)^{T}$ instead of $\left(w^{0}\right)^{1}$.) The mass eigenstoles 
then atiafy $\ddot{x}_{i}^{\circ}=N_{i j} V_{j}(i=1,2,3,4)$. The masses $m_{i j}$ and $m_{\dot{x} ;}$ have been plotted, for typical parameter values, in Fig. J.1b. The neutralino vertex matrices are given by $N$ and $b y^{12}$

$$
\begin{gathered}
O_{i j}^{L}=\|\left(N_{i d} N_{j 4}-N_{i 3} N_{j 3}^{p}\right) \\
O_{i j}^{R^{\prime \prime}}=-O_{i j}^{\prime \prime \prime}
\end{gathered}
$$

\section{The Generul cens}

The general $4 \times 4$ mass matrix may be diagonalized much as in the chargino case. We now must solve a quartic equation. The zeneral roots are eufieiently involved that it he almost always mose expedient ta diagonalize specibc cases numeric ally. Furthermore if a stth neutsalino, such as $N_{0}$ tadded the resulting quintic is typically unsovable in closed form. There are a number of epecial cases which arise which are tractoble anafytically.

\section{$\Delta$ Special Cose}

An example of an instance in which the $x \perp y$ matrix may be readily diegonalized occuss when $v_{1}=v_{2}$ and $M=M^{\prime}$. The various general forms following eqn (1156) then become:

$$
N^{\prime}=\left(\begin{array}{cccc}
1 & 0 & 0 & 0 \\
0 & \cos \omega & \sin \omega & 0 \\
0 & -\sin \omega & \cos \omega & 0 \\
0 & 0 & 0 & 1
\end{array}\right)
$$

where

$$
\tan \omega=\frac{M+\mu-M}{2 M_{z}} \quad \tan 2 \omega=\frac{2 M_{Z}}{M-\mu}
$$

Here

$$
M=\sqrt{(M-\mu)^{2}+4 M}
$$

Bince

$$
B=\left(\begin{array}{cccc}
\cos \theta & \sin \theta & 0 & 0 \\
-\sin \theta & \cos \theta & 0 & 0 \\
0 & 0 & \frac{1}{\sqrt{2}} & -\frac{1}{\sqrt{2}} \\
0 & 0 & \frac{1}{\sqrt{2}} & \frac{1}{\sqrt{2}}
\end{array}\right)
$$

we obtain

$$
N=\left(\begin{array}{cccc}
\cos \theta & \sin \theta \omega & 0 & 0 \\
-\cos \omega \sin \theta & \cos \omega \cos \theta- & \frac{1}{\sqrt{2}} \sin \omega & -\frac{1}{\sqrt{2}} \sin \omega \\
\sin \omega \sin \theta \omega & -\sin \omega \cos \theta & \frac{1}{\sqrt{2}} \cos \omega & -\frac{1}{\sqrt{2}} \cos \omega \\
0 & 0 & \frac{1}{\sqrt{2}} & \frac{1}{\sqrt{2}}
\end{array}\right) .
$$

The mase tizenvalue are

$$
\begin{aligned}
& m_{x i}=M \\
& m_{\bar{X}:}=\langle(M+\mu+M) \\
& m_{\dot{X} 9}=\{(M+\mu-N\} \\
& m_{\bar{x}}=-\mu .
\end{aligned}
$$

To nezate $m_{\bar{X}_{i}^{0}}$ multiply the $\boldsymbol{i}^{\mathbf{k}}$ row of $\boldsymbol{N}$ by $\pm i$ as discussed in the section on charginos. 


\section{FIGURE CAPTIONS}

1. Massts for the indicated supersymmetry-breaking parameters.

a) Charginos

b) Neutralinos
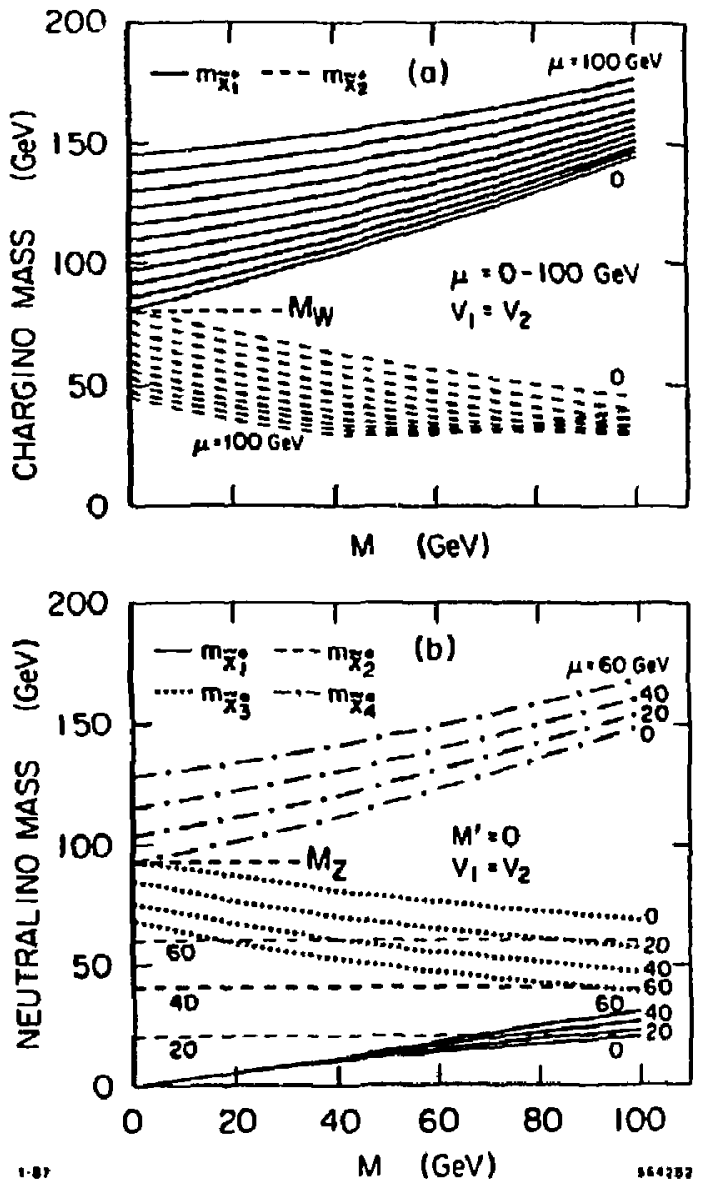

Fig. J.1 


\section{APPENDIX K .}

Feynman Rules

\section{K.I INTRODUCtION}

Given the Lagrangian $\boldsymbol{L}$ it is a etraightforward procen, in principle, to cenerate the Fesnman sules for a theory. The generating functional is

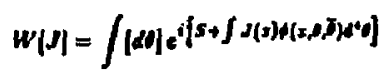

where the action is tiven by

$$
s=\int c(x, 0, \overline{0}) d^{4} x d^{4}
$$

for a cupersymmetric theory. We then generate connected Green's functions vis

$$
G^{(n)}\left(x_{1}, \ldots, x_{N}\right)=\left\langle 0\left|T\left(\phi\left(x_{1}\right) \ldots \phi\left(x_{n}\right)\right)\right| 0\right\rangle=\theta^{n} \frac{\ln \ln w|J|}{\delta J\left(x_{1}\right) \ldots \delta J\left(x_{n}\right)} .
$$

This can be done with the supetfelds directly leading to the so-called cupereraph* approxch. Such a proxedure has proved useful in proving non-renormalization theorems, derivine cuper-Slavnov-Tuylor Identities and so forth but hes limited utility in performing practical calculations when the supersymmetry has teen explicitly brolen. In practice it is far easier to read the Fejnmen rule: directly from $\mathcal{L}$ once it has been fully expanded (zemembering the factor of "i" from (K.1) and any symmetry factors). There is yet another complicalion in the gaugino sector. Because we have Majorane fermions, which carty no conserved internal qusintum number, the direction which a spinor line points is somewhat arbitrary. Using the procedure and construction of refs. 12 and 13 we assign the direction conventionally and establish the corresponding rulea.

\section{K.2 Majorana Fermons}

\section{Charge Conjuration}

The positive and negative-energy solutions to the Dirac equation

$$
(i)-c-m) \psi=0
$$

are $u(b, s)$ and $u(r, s)$ teopectively. It is convenient to define the anti-fermion, $\psi^{4}$, whish is a positive-energy solution to $(i p+c(-m) \psi=0$.

Given (since $\bar{\nabla}=\left(7^{0}\right)$

$$
\begin{aligned}
& \psi=\left(\begin{array}{l}
\varepsilon_{0} \\
\bar{q}^{+}
\end{array}\right) \quad \bar{\psi}=\left(\eta^{0}{\overline{\epsilon_{d}}}_{d}\right) \quad \psi^{\varepsilon}=\left(\begin{array}{c}
\eta_{p} \\
\overline{\varepsilon^{j}}
\end{array}\right)
\end{aligned}
$$

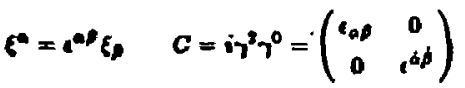

we see that

$$
{ }^{c} c=c \bar{\psi}^{T}
$$

Note the folfowing useful relations:

$$
\begin{gathered}
C^{1}=C^{-1} \quad C=\text { eharge conjucation malrix } \\
C^{\top}=-C \\
C^{-1} \gamma_{s} C=\left(\gamma_{s}\right)^{T} \quad C^{-1} \gamma^{\mu} C=-\left(\gamma^{\mu}\right)^{T}
\end{gathered}
$$




$$
\gamma_{ \pm}=f\left(1 \pm \gamma_{s}\right) \quad \gamma_{3}=\left(\begin{array}{cc}
-1 & 0 \\
0 & 1
\end{array}\right)
$$

For eny uatrix $M,\left(M^{T}\right)^{-1}=\left(M^{-1}\right)^{T}$, in perticuler

$$
\left(c^{T}\right)^{-1}=\left(C^{-1}\right)^{T}
$$

$$
\begin{aligned}
& v^{T}(k, 1)=v(k, 0) C^{T} \quad \nabla^{\top}(k, 0)=\pi(k, 0) C^{T} \\
& \mathbb{v}^{\top}(k, v)=C^{-1} v(k, 0) \quad v^{\top}(k, v)=C^{-1} v(k, d) \\
& \gamma^{\mu} \gamma_{ \pm}=\gamma_{\mp} \gamma^{\mu} \quad\left(\gamma_{ \pm}\right)^{\mu}=\gamma_{ \pm} \quad \gamma_{ \pm} \gamma_{\mp}=0
\end{aligned}
$$

(K.11)

In any process we attach apinor lezs according to

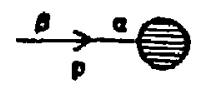

$\forall(p)$ op

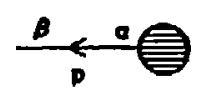

viploe

प(p) po

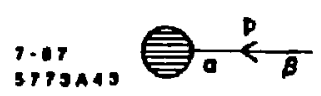

$v(p)_{0}$

(K.13) 


\section{Majorane Propagators:}

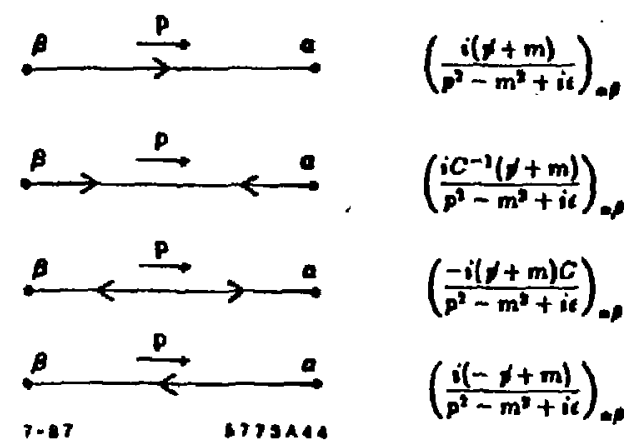

Vertex Minnipulation

Let $\lambda_{1} \psi_{2} c$ be a general Misjorans sermion, Ditac fermion and boson fild ro spectively, converting al a single vertex. Then ${ }^{12}$ the valous permutations of the direction arrows and correspondine sules are illustrated in Fit. K.1. The amblEuilies arise because Mijorane fermions casy no conserved quantum number.

\section{K.3 Chargino Ventices}

Let $e_{1,}$ inditetc a representative lepton doublet with no family mixine (artow lndicate the fow of positive charge)." We ignore $m_{e}$ since in the matrix element if is megligible zelative $10 \mathrm{~m}_{3}$. The verlex is

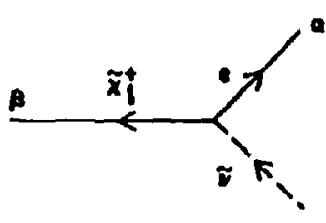

$\left.\mid-i p v_{j 1} \gamma_{+} C\right]_{a p}$

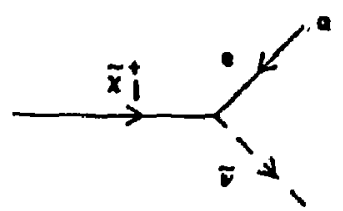

B773A4B

Where $V_{10}$ wes defined in Appendices 1 and 3 .

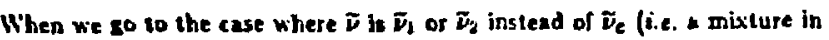
flavor space) we will in addition have mixing angles $\left(\sin D_{v}, \cos D_{4}\right)$ in the vestex (in general when all 3 generators mix, we will have a mixing matrix element). 
To see how sotne of the above rules work we will derive two new effective rules lor the previous vertices when the Direc lepton (e) is an external particle and the correspondine epinor has nol been truncated. We will go direelly to the case of $\mu$ - r mixins.

$\tilde{\nu}_{1}=\tilde{\nu}_{p} \cos \theta_{v}+\tilde{\nu}_{r} \sin \theta_{v} \quad \tilde{\nu}_{2}=-\tilde{\nu}_{w} \sin \theta_{v}+\tilde{\nu}_{r} \cos \theta_{p}$

(K.15)

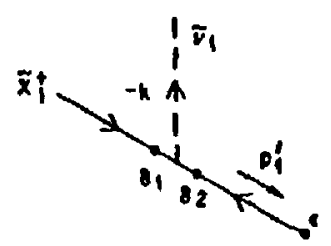

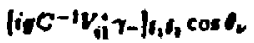

$7: 17$

$$
\left[v+\left(p_{1}^{\prime}\right)\right]_{2}
$$

This port of the matrix eleroent is

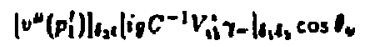

$=i g \cos \theta_{v}\left(\mid v^{*}\left(p_{1}^{\prime}||^{T}\right)_{c_{1}} v_{i i}\left(\left|c^{-1} \gamma-\right|^{T}\right)_{d_{2} S_{1}}\right.$

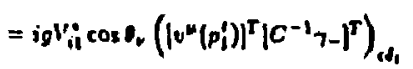

$=i g V_{i j} \cos C_{v}\left(\sigma^{\mu}\left(p_{i}^{\prime}\right) C^{T} \mid(7-)^{T} C^{-1} V^{T}\right)_{\text {d, }}$ using $(K .9)$ and $(K .12)$

$=i g V_{i j} \cos \theta_{r}\left(\sigma^{n}\left(p_{i}^{p}\right) C^{T}\left(C^{-3}\right)^{T_{-}}\right)_{d_{1}}$

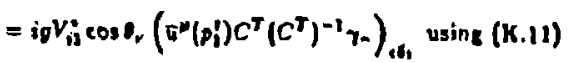

$=i g V_{i 1}^{i} \cos \theta_{v}\left(E^{\prime \prime}\left(G^{\prime}\right) r-\right)_{d b_{1}}$
Now regading Fig. K.1 we can graphically depict this a

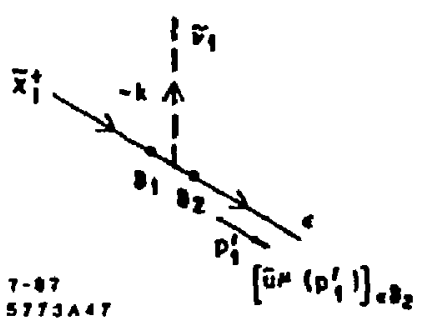

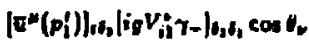

(K.18)

Thus (K.18) is * new effective non-truncated vertex (which will prove useful). Similarly, from (K.14) the conjugate of the previous example sives us

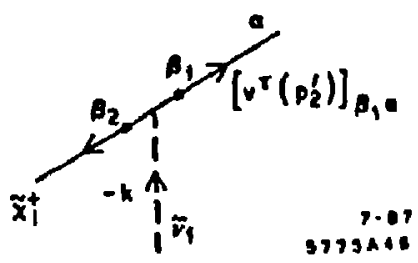

$\left.\mid-i_{\theta} v_{i 1} \gamma_{+} C\right)_{\rho_{1} \rho_{1}} \sin \varphi_{*}$

(K.10)

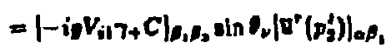

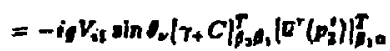

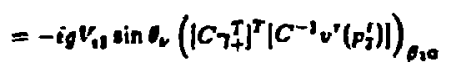

$=-i g v_{i 1} \sin \theta_{v}\left({ }_{7} C^{T} C^{-1} v^{\top}\left(p_{3}^{\prime}\right)\right)_{p, 0}$

$=-i g V_{13} \sin t_{v}\left(7+(-C) C^{-1} v^{\prime}\left(p_{2}^{\prime}\right)\right)_{\theta_{1}}$

$=\operatorname{lig}_{1} V_{11} \sin \theta_{2}+\left.v^{\prime}\left(p_{3}^{\prime}\right)\right|_{g_{2} \alpha}$ 
Depicted etaphically a

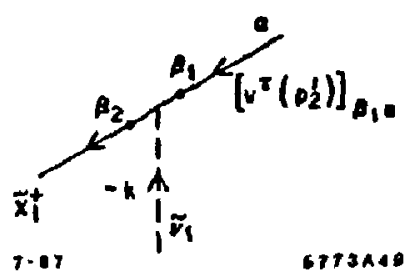

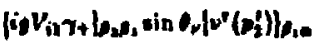

It wuat be emphesized that (K.11) and (K.21) are valid only for the opecific case where the lepton ( $\mu$ or 1$) \mathrm{L}(1)$ external and (2) in the final state (as in our problem). In general (K.16) and (K.19) must be und. We will relet to (K.18) and (K.21) a "derived" Fejnman rules and use them to great efiect in the next eppendix.

\section{Other "chargino" verlices}

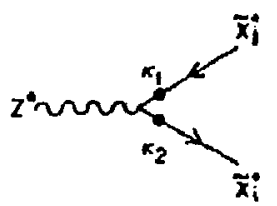

$7 \cdot 0$

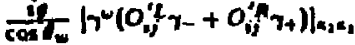

(K.22)

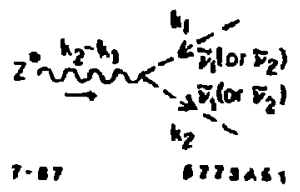

$\frac{-i g}{\cos t_{w}}\left|k_{2}+k_{2}\right|$

$\frac{i \theta^{2}}{2 \cos t_{w}}\left|\gamma^{\omega}\left(\cos 2 \theta_{\omega} \gamma_{-}-2 \sin ^{2} \theta_{w} \gamma_{+}\right)\right|_{\alpha_{1} \alpha_{2}}$

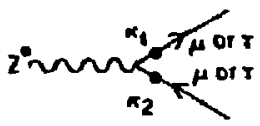

(h'.24)

$=i \varepsilon\left\{\gamma^{\sim}\left(\cos 2 \theta_{w} \gamma_{-}-\tan \omega_{w} \gamma_{t}\right)\right\}_{\kappa_{1} \alpha_{2}}$ 
K.4 NeUtaAlno Vertices

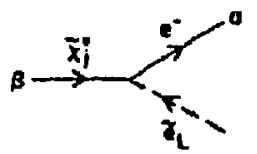

t.6 crosas

\section{$\frac{i \theta}{\sqrt{2} \cos \theta_{0}} \sin 2 \theta_{-} N_{j 1}^{\prime}+\cos 2 \theta_{-1} N_{j 2}^{\prime} \mid{ }_{t}$}

(K.25)

With no fasully mixing.

We wish to express our results in terms of $N_{i j}$ ( $N$ and $N^{\prime}$ were defined in Appendix I).

$$
N_{j 1}^{\prime}=N_{j 1} \cos \theta_{0}+N_{j 2} \sin \theta_{w} \quad N_{j 2}^{\prime}=-N_{j 1} \sin \theta_{0}+N_{j 1} \cos \theta_{0} \quad(K .26)
$$

The sbove verlex becomes

$$
\begin{aligned}
& v_{x^{\prime}, 1}=\frac{i j}{\sqrt{2} \cos \theta_{\omega}}\left[2 \sin \theta_{\nu} \cos \theta_{\psi}\left(N_{j 1} \cos \theta_{\omega}+N_{j 2} \sin \theta_{\nu}\right)\right. \\
& +\left(\cos ^{2} \theta_{v}-\sin ^{2} \theta_{\omega}\right)\left(-N_{j 1} \sin \theta_{w}+N_{j 2} \cos \theta_{\omega}\right) \gamma_{+} \\
& =\left\{\frac{i g}{\sqrt{2}}\left|\operatorname{anc} \theta_{-} N_{j 1}+N_{j 3}\right| \gamma_{+}\right\}_{a p}
\end{aligned}
$$

Therelore using Fi. K.I

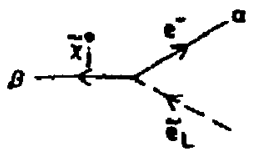

$7 \cdot 01$ $\left\{\frac{i}{\sqrt{2}}\left|\tan \varphi_{+} N_{j i}+N_{j 3}\right| \tau_{+} C\right\}_{e}$

(K.28)

\section{Reversinc all errowe in (K.28) yields}

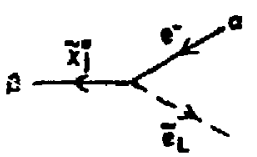

$\overline{\frac{d}{\sqrt{b}}\left[\operatorname{lan} C_{-\infty} N_{j 1}+N_{j l} \mid{ }_{+} C\right\}_{\alpha p}}$

(K.29)

tit eTras

$=\left\{\frac{-i}{\sqrt{3}}\left|\ln \theta_{0} N_{j 1}+I_{j j}\right| C^{-1} \gamma-\right\}_{\rho 0}$

\section{Acain from Fig. K.1}

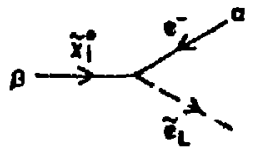

$=\left\{\frac{-i f}{\sqrt{2}}\left|\tan \omega_{-\infty} N_{j 1}+N_{j 2}\right| C^{-1} 7-\right\}_{D 0}$

7.07571345 
Similarly for the $\tilde{x}_{j}^{0} \tilde{t}_{A}$ vertex

$$
\begin{aligned}
& \frac{i}{\sqrt{2}}\left[\rho \sin \omega_{w} N_{j i}^{\prime}-\frac{\sin ^{2} \omega_{\omega}}{\cos \theta_{\omega}} N_{j i}^{\prime}\right](1-r) \text {. } \\
& =\frac{2}{\cos \phi_{\omega}}\left(\frac{-i \rho}{\sqrt{2}}\right)\left\{\sin \theta_{\omega} \cos \theta_{\omega}\left(N_{j}^{\prime \prime} \cos \theta_{\omega}+N_{j 2}^{i} \sin \theta_{w}\right)\right. \\
& -\sin ^{2} \theta_{w}\left(-N_{j 1} \sin \theta_{w}+N_{j 2}^{k} \cos \theta_{w}\right) \gamma- \\
& =\left\{-\frac{i e \sqrt{2}}{\cos \theta_{6}} N_{j l} \gamma-\right\}_{0}
\end{aligned}
$$

so the vertex is

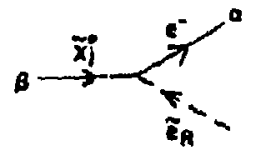

3.07 แ170467

$$
\begin{array}{r}
\left\{-\frac{\operatorname{it} \sqrt{2}}{\cos N_{j k}^{1}-}\right\}_{a} \\
\text { (K.31) }
\end{array}
$$

Lting Fiz. K.I we obtain these equivalent vertices:

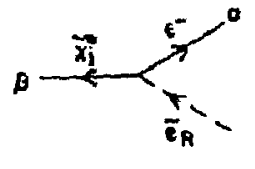

$$
\left\{-i g \sqrt{2} \tan N_{w i} \gamma-C\right\}_{0 \alpha}
$$

7.87

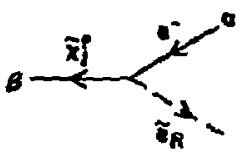

$2 \cdot 8$

แTJ3ASE

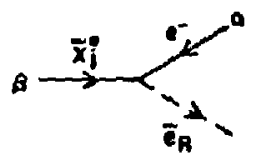

$1 \cdot 4$

119450

$\left\{\operatorname{ig} \sqrt{2} \tan D_{w} N_{j p} C^{-1} \gamma_{+}\right\}_{\% a}$

In all cases above the rules bave been demontrated for no alevor mixing. (i.c.

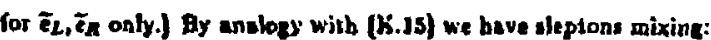

$$
\begin{aligned}
& \bar{t}_{L L}=\tilde{\mu}_{L} \cos \theta_{L}+\tilde{\tau}_{L} \sin \theta_{L} \\
& \bar{l}_{2 L}=-\tilde{\mu}_{L} \sin \theta_{L}+\bar{\tau}_{L} \cos \theta_{L} \\
& \tilde{\ell}_{A}=\tilde{\mu}_{R} \cos \theta_{R}+\tilde{T}_{A} \sin \theta_{A} \\
& \tau_{2 A}=-\tilde{\mu}_{A} \sin \theta_{A}+\tilde{T}_{R} \cos \theta_{A}
\end{aligned}
$$

In the simplest cuse

$$
\boldsymbol{U}_{\boldsymbol{r}}=\boldsymbol{\omega}_{\boldsymbol{L}}=\boldsymbol{\omega}_{R} \equiv \boldsymbol{\bullet}
$$




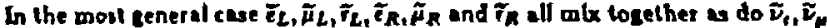
and $\nabla_{r}$. This leads to rules like:

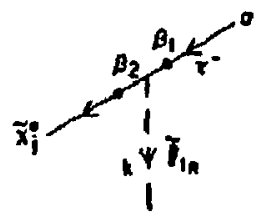

$\operatorname{lig} \sqrt{2} \tan \theta_{-} N_{j t} \gamma+\left.\right|_{n,} \theta_{1} \operatorname{tin} \theta_{R}$

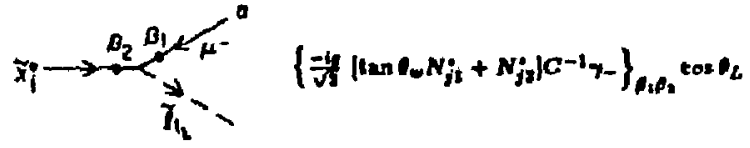

$7 \cdot \mathbf{7}$

Groses
Other "xieutraline" Verlices

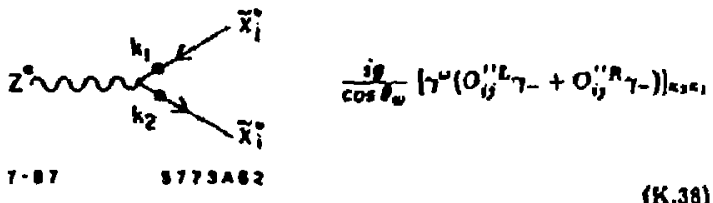

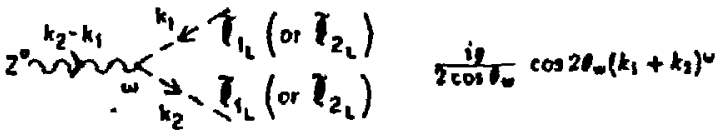

$$
\begin{aligned}
& \text { T.17 SPY3A6J (K.39) }
\end{aligned}
$$

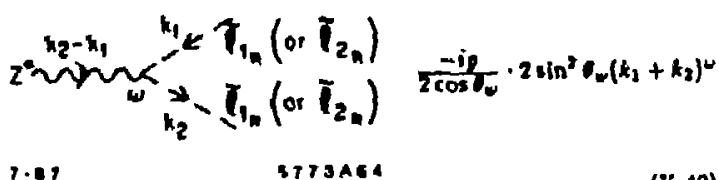

These are cll of the Feynman rules required for the process $z^{0} \rightarrow \mu^{-} e^{+}$, 


\section{FIGURE CAPTIONS}

1. The various permutations of the direction anrows and corresponding sules for a vertex consinting of $\lambda, \phi, c$ which are general Majorana fermion, Dirac fermion and boson beld respectivaly.

Here

$$
r_{i}=1, i_{5,} \gamma_{\mu} \gamma_{S}, \gamma_{\mu}, \sigma_{\mu \nu} .
$$

Fig. K.1
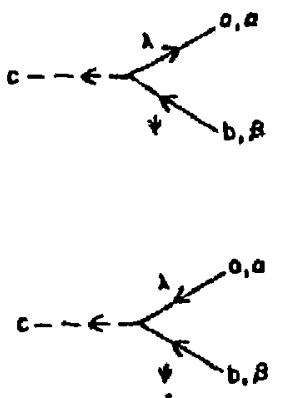

$-i K_{\text {suc }}^{i}\left(C^{-1} \Gamma_{i}\right)_{a p}$

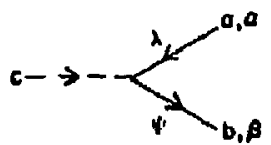

iKikis $r_{\text {iso }}$

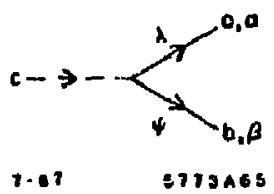

$i K_{\mathrm{it}}\left(\mathrm{r}_{\mathbf{1}} \mathrm{C}\right)_{\text {po }}$ 


\section{APPENDIX L}

Compulation of $z^{\circ} \rightarrow$ s $\mu$ Matrix Elements

\section{L.1 Chargino Diagrams}

\section{Diariam 1}

We demonstrale the use of the "manipulated" Feynman rules (K.18) and (K.21) here. Disgram one is illustrated in Fig. 1. From the Fejnman rules of Appendix $K$ we may read of the matrix element:

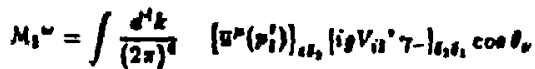

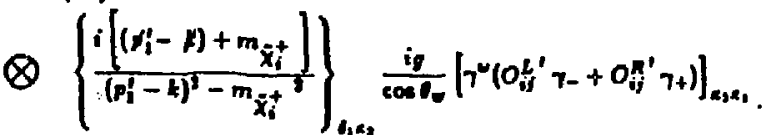

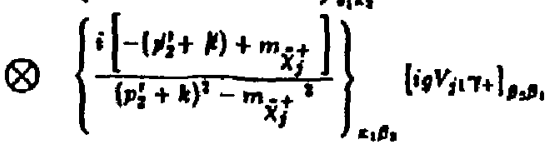

$$
\begin{aligned}
& \text { Q } \sin t_{\nu} \frac{i}{k^{i}-m_{\nu_{1}}^{2}}\left[v^{\prime}\left(p_{j}^{\prime}\right)\right)_{\theta_{1} \circ} \\
& =\frac{-g^{3} \sin \theta_{\nu} \cos \theta_{2} v_{j 1} v_{i i^{\prime}}}{\cos \theta_{\sigma}} \int \frac{d^{\mu} k}{(2 \pi)^{4}}
\end{aligned}
$$

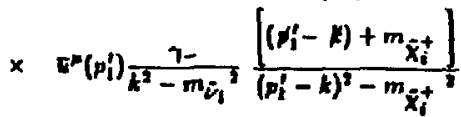

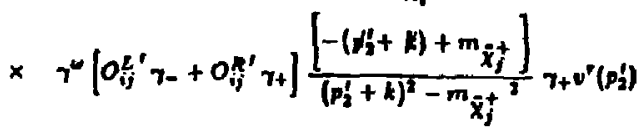

Notice how simple the rastrix index etructure is. This is a result of using the manipulated rules (K.18) and (K.21). It these had not been utilized we would essentially have derived them when simplifying the expression which would have resulled.

$$
\begin{aligned}
& M_{1}^{*}=\frac{g^{3} \sin \theta_{w} \cos \theta_{v} V_{j 1} V_{i i^{*}}}{\cos \theta_{0}} \int \frac{d^{4} k}{(2 \pi)^{4}}
\end{aligned}
$$

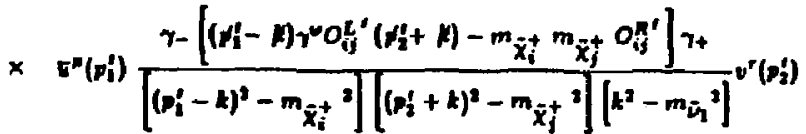

where we have used

$$
\gamma^{\mu} \gamma_{ \pm}=7 \mp \gamma^{\mu} \quad\left(7_{ \pm}\right)^{2}=\gamma_{ \pm} \quad 7_{ \pm 7 \neq}=0
$$

several times. Uning

$$
\frac{1}{k^{1}-m_{\dot{\nu}_{2}}^{2}}-\frac{1}{k^{2}-m_{i_{3}}^{2}}=-\int_{m_{b_{1}}}^{m_{b^{\prime}}} \frac{d t}{\left(k^{2}-t\right)^{2}}
$$

we obtain

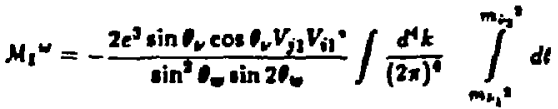

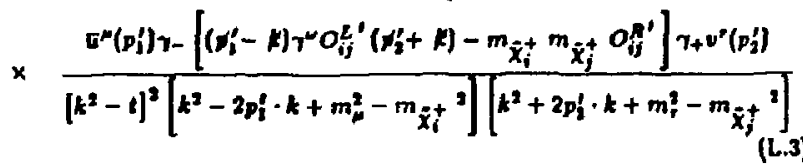

We proceed to ignore all $m_{\mu,}^{2} m_{i}^{2}$ terms (relative to $\rho^{2}, m_{\bar{\nu}_{1}}^{2}, m_{\tilde{x}_{i}^{+}}^{2}$ etc. terms). 
From equation (H.s)

$$
\frac{1}{a^{2} b c}=0 \int_{0}^{1} d x \int_{0}^{1-a} d y \frac{1-x-y}{[a+(b-e) x+(c-c) y]^{4}}
$$

Therefore

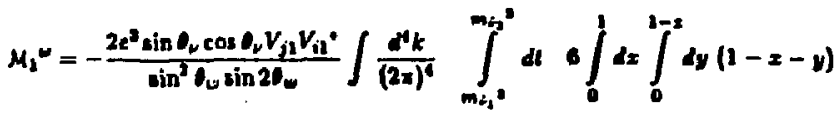

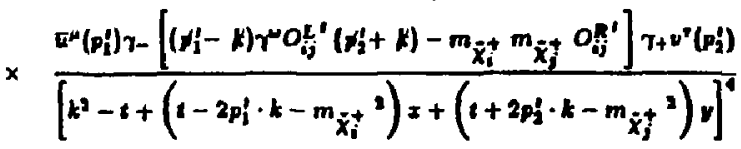

(L.4)

From (H.10) we can twitch variables of integration.

$$
\sum x=y \quad z=x-y
$$

so that

$$
\begin{gathered}
x y=\frac{1}{4}\left(x^{2}-z^{2}\right) \\
\int_{0}^{1} d x \int_{0}^{1-2} d y=1 \int_{0}^{1} d z \int_{-=}^{1} d z
\end{gathered}
$$

We naw employ the formulat of (H.7)

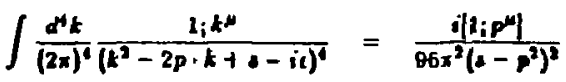

$$
\begin{aligned}
& \int \frac{d^{4} k}{(2 x)^{4}} \frac{k k_{\mu} k^{\nu}}{\left(k^{2}-2 p \cdot k+a-i c\right)^{4}}=\frac{i \mid\left(p_{\mu} p^{\nu}+\left|\left(4-p^{2}\right) \delta_{\mu}^{\mu}\right|\right.}{D 6 x^{2}\left(a-p^{2}\right)^{3}}
\end{aligned}
$$

to recest ous Integtal lato the form

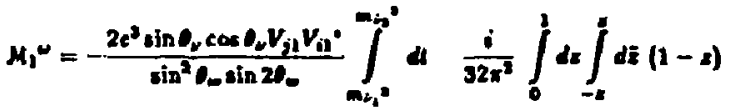

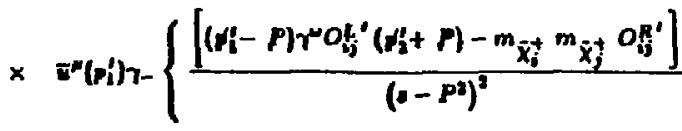

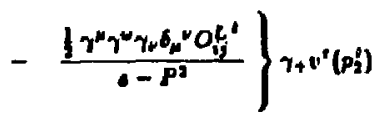

(L.8)

with

$$
\begin{aligned}
& P=p i x-p i y \\
& s=-1(1-x-y)-\left[m_{\bar{x}_{i}^{+}}{ }^{2} x+m_{\bar{x}_{j}^{+}}{ }^{2} y\right] \\
& =-\left\{1(1-s)+1\left[\left(m_{\bar{x}_{i}^{+}}^{2}+m_{\bar{x}_{j}^{+}}^{2}\right) x+\left(m_{\bar{x}_{j}^{+}}^{2}-m_{\bar{x}_{j}^{+}}^{2}\right) \bar{z}\right]\right\}
\end{aligned}
$$

As in (H.17 a) we see that

$$
P^{2} \doteq \frac{1^{2}}{1}\left(\dot{z}^{2}-x^{2}\right)
$$

and so

$$
\begin{aligned}
& -P^{2} \equiv-D(t) \\
& =-\left(x(1-2)+1\left[\left(m_{\tilde{x}_{i}^{+}}^{2}+m_{\tilde{x}_{i}^{+}}^{2}\right) z+\left(m_{\tilde{x}_{i}^{+}}^{2}-m_{\hat{x}_{j}^{+}}^{2}\right) i\right]\right. \\
& \left.+\frac{2^{2}}{4}\left(z^{2}-x^{2}\right)\right\} \text {. }
\end{aligned}
$$

(L.7)

261

262 
From (L.3)

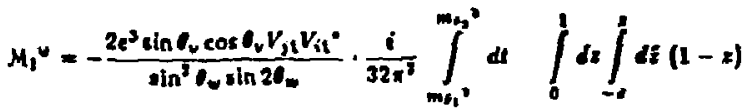

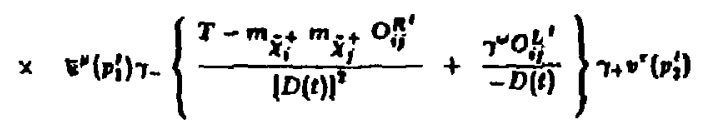

since $T^{\prime \prime} \gamma^{\omega} \gamma_{\mu}=-2 \gamma^{\omega} \cdot \ln (L .8)$

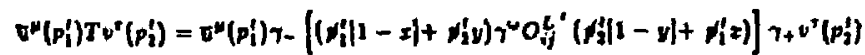

Using the Dirsc equation

$$
\vec{u}^{\prime \prime}\left(p_{i}^{\prime}\right) \| \sim H_{i}^{\prime} v^{\prime}\left(p_{2}^{\prime}\right) \approx 0
$$

this becomes

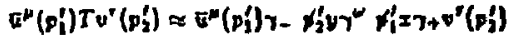

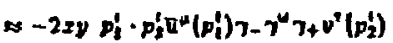

$$
\begin{aligned}
& \approx \frac{1}{i} 0^{2}\left(i^{2}-x^{2}\right) \epsilon^{*} c
\end{aligned}
$$

where we recall from $(3.9$ a) that

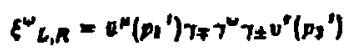

Also retall (from (3.9 d) that

$$
K_{v}=\frac{i}{16 x^{2}} e^{2} \sin e_{\nu} \cos \theta_{v}
$$

and

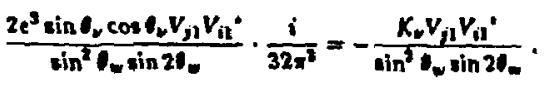

Perforning the $t$ integration and substituting for $T$ in $(L .8)$

$$
\begin{aligned}
& M_{1}{ }^{u}=\sum_{i j} \varepsilon_{L} \frac{K_{2} V_{j 1} v_{i 1}}{\sin ^{2} \theta_{-} \sin 2 l_{0}} \int_{0}^{1} d z \int_{-1}^{\infty} d i
\end{aligned}
$$

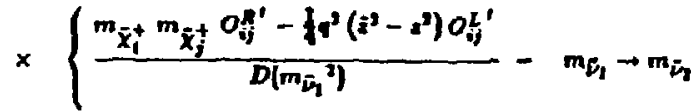

$$
\begin{aligned}
& \left.+o_{j}^{\prime \prime} \ln \left[\frac{D\left(m_{\bar{\nu}_{2}}{ }^{\prime}\right)}{D\left(m_{\bar{\nu}_{1}}{ }^{\prime}\right)}\right]\right\}
\end{aligned}
$$

where

$$
\begin{aligned}
& D\left(m_{i_{1}}{ }^{3}\right)=m_{i_{1}}{ }^{2}(1-x) \\
& \left.+1\left[\left(m_{\bar{x}_{i}^{+}}{ }^{2}+m_{\bar{x}_{j}^{+}}{ }^{2}\right)=+\left(m_{\bar{x}_{i}^{+}}{ }^{2}-m_{\dot{x}_{j}^{+}}\right)\right]\right\}+\frac{1}{i} i^{2}\left(z^{\prime}-2^{2}\right)
\end{aligned}
$$

U we had employed a traditional Pauli.Villars reqularization echeme we would have obtained

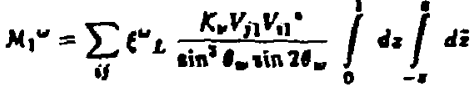

$$
\begin{aligned}
& \times\left\{\frac{m_{\tilde{x}_{i}^{+}} m_{\dot{x}_{j}^{+}} O_{i j}^{R^{\prime}}-l_{i}^{2}\left(\dot{z}^{2}-z^{\prime}\right) O_{i j}^{L}}{D\left(m_{\dot{i}_{2}}{ }^{\prime}\right)}\right.
\end{aligned}
$$

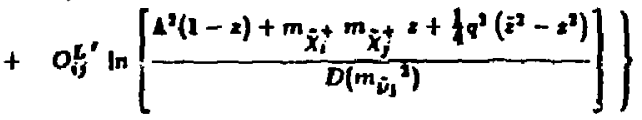

$$
\begin{aligned}
& \text { - } m_{\tilde{y}_{1}} \rightarrow m_{\bar{y}_{2}}
\end{aligned}
$$

Hese $A^{1} \rightarrow \infty$ is the Pauli-Villare regulator which is superfuous in this instance 
since we have equally well written the $\log$ term in (L.12) as a ratio of "D's: where the numerator " $D$ " is equal to the denominate with with $\tilde{\nu}_{1}$ replaced by $\dot{i}_{2}$. Dividing (l.12) through by $m_{\bar{x}_{i}^{+}}{ }^{2}$ and using the definitions of the ration $R, s$ and $T$ from ep. ( 0.10$)$ and the integral functions in the table at the conclusion of the appendix (Table L.1) gives us the final form

$$
\begin{aligned}
& \mu_{1}{ }^{*}=\sum_{i j} f_{L}^{\omega} \frac{2 x_{2} v_{i 1} v_{i j}{ }^{*}}{\sin ^{2} \theta_{\omega} \sin 2 u_{\omega}} \\
& \times\left\{\sqrt{S_{i j}^{+}} O_{i j}^{\prime \prime} \Delta I_{(j)}\left(T_{i}^{+}, s_{i j}^{+}, R_{i}^{+}\right\}\right. \\
& \left.-R_{i}^{+} O_{i j}^{L} \Delta I_{[\overline{2}-2\}^{\prime} \mid}\left(T_{i}^{+}, s_{i j}^{+}, R_{i}^{+}\right)+O_{i j}^{L^{\prime}} \Delta G\left(T_{i}^{+}, S_{i j}^{+}, R_{i}^{+}\right]\right\}
\end{aligned}
$$

Diagram 2

Diagram two is illustrated in Fig. 2. From the Feynman rules of Appendix K we may read of the matrix element:

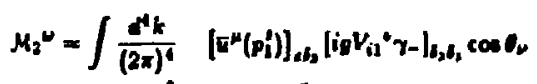

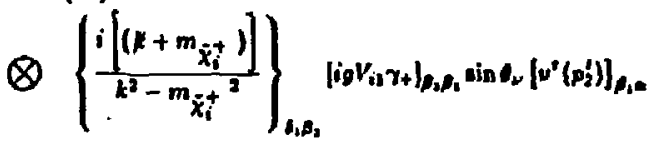

$\otimes \frac{i}{\left(p_{i}^{\prime}-k\right)^{2}-m_{i_{1}}^{2}} \cdot \frac{-i g^{2}}{2 \cos 0_{\omega}}\left[p_{i}^{\prime}-p_{i}^{\prime}-2 k\right]^{\omega} \cdot \frac{i}{\left(p_{i}^{\prime}+k\right)^{2}-m_{i_{1}}^{2}}$

$$
\begin{aligned}
& =\frac{e^{2} \sin \theta_{v} \cos \theta_{w} v_{i 1} V_{i j}}{2 \cos \theta_{w}} \int \frac{d^{4} t}{(2 \pi)^{4}}
\end{aligned}
$$

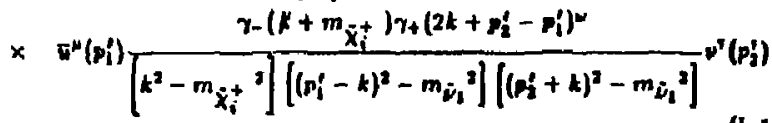

(L.14)

We see that use of (K.38) and (K.21) have again simplified the algebra. In this
- case ne use a Pauli-Villare cut-of. Introducing

$$
\frac{1}{k^{2}-m_{\bar{x}_{i}^{+}}^{2}}=-\int_{m_{\bar{x}_{i}^{+}}^{*}}^{t^{*}} \frac{d}{\left(t^{2}-t\right)^{2}}
$$

Following the techniques used above we obtain

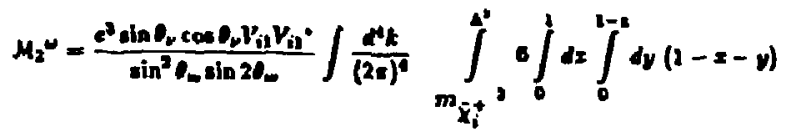

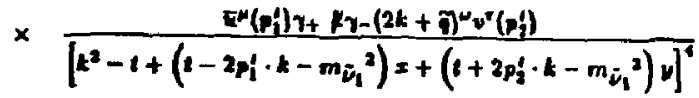

$$
\begin{aligned}
& -\bar{w}_{1} \rightarrow \boldsymbol{C}_{1}
\end{aligned}
$$

where

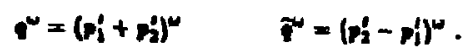

This leads to

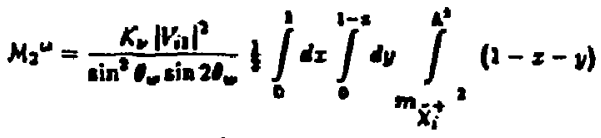

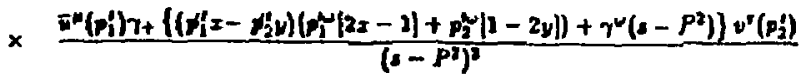

$$
\begin{aligned}
& -\bar{\nu}_{1} \rightarrow \bar{\nu}_{2}
\end{aligned}
$$

viliere

$$
\text { s- } P^{2} \approx-t(1-s)-m_{i_{1}}{ }^{2} z-\frac{q^{2}}{4}\left(\bar{z}^{2}-z^{2}\right) \text {. }
$$

265

206 
The terms will intsoduce factors of $m_{\mu,} m$, and so will be down by

$$
O\left(m_{r} / m_{s u s r}\right)
$$

relative to the $\boldsymbol{\gamma}^{\mathrm{w}}$ term. Thus

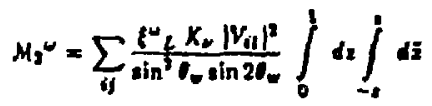

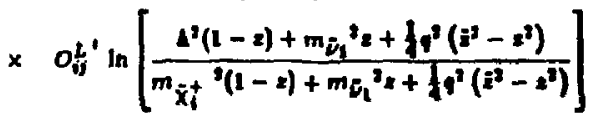

$$
\begin{aligned}
& -m_{p_{1}} \rightarrow m_{n_{1}}+0\left(\frac{m_{r}}{m_{s v_{Y}}}\right)
\end{aligned}
$$

and because, an we shall seen the colal matrix element is thite as $\Lambda^{x} \rightarrow \infty$ we may write this sans regulatiation as

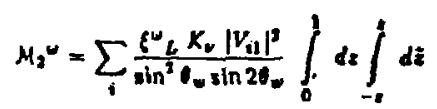

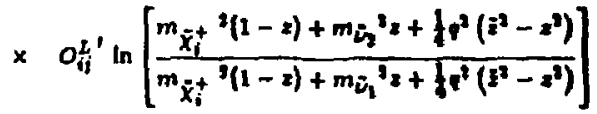

$$
\begin{aligned}
& +o\left(\frac{m_{1}}{M_{\text {sUSY }}}\right)
\end{aligned}
$$

Where $K_{v}$ is siven by (L.1t) and $f_{L}$ by (L.10). Dividing through by $m_{\bar{x}_{i}^{+}}$, and using the definition of the Integrat functions in the table at the conelusion of this appendix yields

$$
M_{2}^{*}=\sum_{i} \frac{\xi_{L}^{*} K_{v}\left|V_{i 1}\right|^{2}}{\sin ^{2} \theta_{\omega} \sin 2 l_{\omega}} \Delta C\left(T_{i}^{+}, A_{i}^{+}\right)
$$

\section{Diaeram Three}

Diagrams three and four are leg corrections. For these two ets of diegtams we will nos use (K.18) and (K.21) in an effort to deroongiate some of the C-batilx manipulations which occur. From Fic. 3 we see that

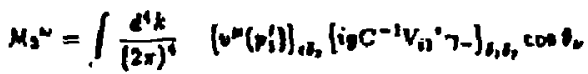

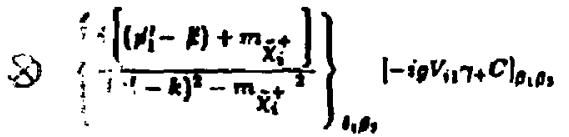

$$
\begin{aligned}
& Q \operatorname{in} c_{\nu} \frac{i}{k^{2}-m_{n_{1}}^{2}}\left\{\frac{i\left(-\mu i+m_{i}\right)}{\left(b_{i}\right)^{2}-m_{i}^{2}}\right\}_{k, \ldots}
\end{aligned}
$$

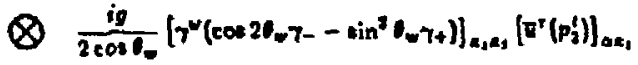

Now we take a few transposes in order to plece this in the form of a matrix product in the upin indicies.

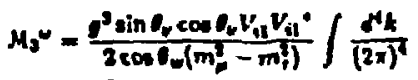

$$
\begin{aligned}
& \times\left[u^{\prime \prime}\left(p_{i}^{\prime}\right)\right]^{r}\left[c^{-1} \eta_{-}\right]^{\top} \frac{\left[\left(H_{i}-k\right)+m_{\bar{x}_{i}^{+}}\right.}{\left(p_{i}^{\prime}-k\right)^{2}-m_{\bar{x}_{i}^{+}}} \\
& \times(1+c)^{T}\left[-H_{i}+m_{r}\right]^{T} \frac{1}{k^{T}-m_{i_{2}}} \\
& \times\left[\gamma^{N}\left(\cos 2 \omega_{-\gamma}-\sin ^{2} \theta_{-\gamma_{+}}\right)\right]^{T}\left[u^{s}\left(p^{\prime}\right)\right]^{T}
\end{aligned}
$$

We now proced to use the various identities given the betinning of Appendix K. 
Since $C^{-1} \gamma_{-}=\gamma_{-}^{\top} C^{-1}$ and $\gamma_{+} C=C \gamma_{+}^{\top}$ using (K.12)

$$
\begin{aligned}
& M_{2}{ }^{*}=\frac{e^{2} \sin O_{2} \cos O_{2} V_{i 1} V_{i j}}{2 \cos O_{w}\left(m_{m}^{2}-m_{i}^{2}\right)} \int \frac{N k}{(2 \pi)^{4}} \\
& \times\left[\bar{u}^{\mu}\left(p_{i}^{\prime}\right) C^{\top}\right]\left[\gamma^{r} C^{-1}\right]^{T} \frac{\left[\left(H_{i}-\not\right)+m_{\bar{x}_{i}^{+}}\right.}{\left(p_{i}^{\prime}-k\right)^{2}-m_{\dot{x}_{i}^{+}}^{2}} \\
& \times\left[C_{1}^{T}\right]^{T}\left[-H_{i}^{\prime}+m_{l}\right]^{T} \frac{1}{k^{2}-m_{\nu_{2}}^{2}}
\end{aligned}
$$

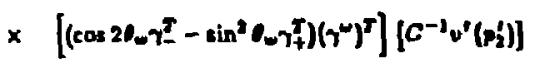

(L.21)

$$
\left(\gamma^{2} c^{-1}\right)^{T}=\left(C^{T}\right)^{-1} \gamma
$$

and using (K.8)

$$
\left(C r_{+}^{T}\right)^{T}=-\gamma_{+} C
$$

$\infty$

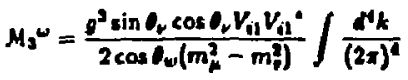

$$
\begin{aligned}
& x \quad \bar{u}^{\mu}\left(p_{i}^{\prime}\right) c^{\top}\left(c^{x}\right)^{-1} \gamma-\frac{\left(\left(p_{i}^{\prime}-k\right)+m_{\hat{x}_{i}^{+}}\right]}{\left(p_{i}^{\prime}-k\right)^{2}-m_{\hat{x}_{i}^{+}}^{2}} \\
& \left.\times\left|-\tau_{+} C\right| \mid-H_{i}+m_{r}\right]^{T} \frac{1}{k^{2}-m_{i_{1}}{ }^{2}} \\
& \times\left(\cos 2 \omega_{\omega} \tau-\sin ^{\top} \theta_{\omega} \tau_{+}^{T}\right)\left(\gamma^{\omega}\right)^{T} C^{-1} \nu^{\top}\left(p_{2}^{\prime}\right)
\end{aligned}
$$

We now "slide" the $C^{-1}$ neat the end of $(L .22)$ until it combines with \& $C$ via
$C C^{-1}=1$ and repeatedly use (K.11) to obtain

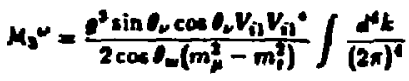

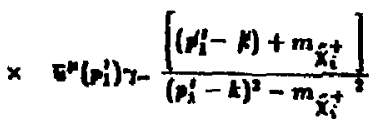

$$
\begin{aligned}
& \times\left(-q_{+}\right)\left(H_{i}^{\prime}+m_{l}\right) \frac{1}{h^{2}-m_{\hat{\nu}_{l}}^{2}} \\
& \times\left(\cos 2 t_{\omega \gamma} \gamma-2 \sin ^{2} \omega_{\omega} \gamma_{+}\right)\left(-\gamma^{2}\right) v^{\prime}\left(p_{2}^{\prime}\right)
\end{aligned}
$$

and since $\gamma_{ \pm} \gamma_{\boldsymbol{F}}=0$ and $\gamma_{ \pm} \gamma_{ \pm}=\gamma_{\sharp}$

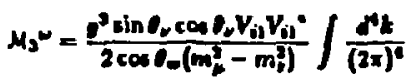

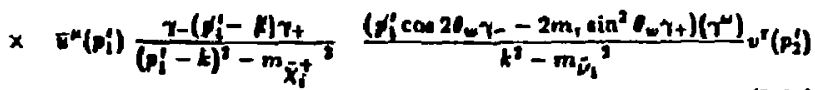

We proceed as we did for the previous diagram, introducing a Pauli-Villare regulster vie $f \mathcal{A}$, but use (Hos) instead of (H.S). We find (after eliminating the regulator)

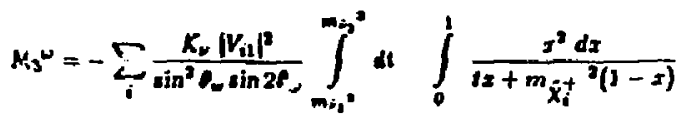

$$
\begin{aligned}
& \times \frac{\cos 20_{\omega} m_{L}^{2} t_{L}-2 \sin ^{2} O_{L} m_{\mu} m_{r} t_{R}^{\omega_{R}}}{m_{R}^{2}-m_{F}^{2}} .
\end{aligned}
$$

from Table L.I we see that this integral is lite $G(T, R)$ when $R=0\left(\right.$ ie. $\left.q^{2}=0\right)$.

20

270 
Therofore we can write this as

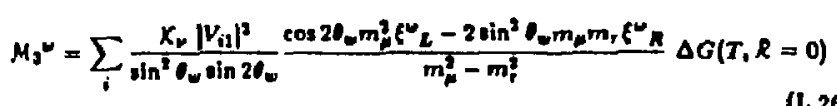

\section{Dierrera Four}

Diagram four is computed in precisely the ame manner as the previous contribution From Fig. 4 we may write

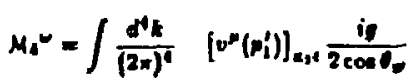

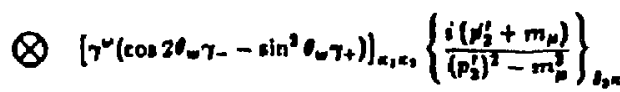

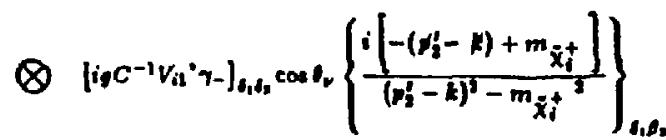

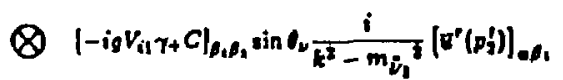

Following the same ateps leads us to

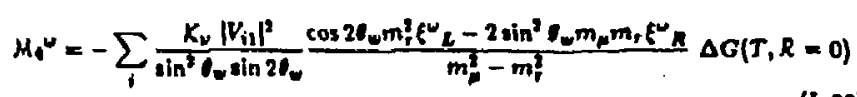

From (L.26) and (L.28) we conclude

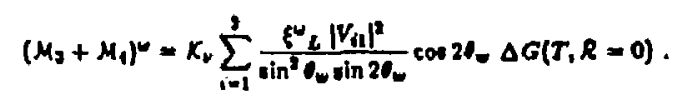

Nots that the $t_{a}$ term hese completely cancelled between the iwo les correction diacrouss.

\section{Total Chargino Matrix Element}

We may now gather together the individual contributions and present the complete chas gino matrix elevent. From (L.13), (L.18) and (L.29)

$$
\begin{aligned}
& \mu_{ \pm} \omega^{\prime}=2 K_{\nu} \epsilon_{L} \sum_{i j=1}^{2} \frac{v_{j 1} v_{i 1}}{\sin ^{2} \theta_{-\sin 2 \theta_{\omega}}} \\
& \times \quad \sqrt{S_{i j}^{+}} o_{i j}^{R^{\prime}} \Delta I_{[3]}\left(T_{i}^{+}, s_{i j}^{+}, R_{i}^{+}\right) \\
& \left.-R_{i}^{+} O_{i j}^{L^{\prime}} \Delta I_{\left\{Z_{1}-2,\right.}\left(T_{i}^{+}, s_{i j}^{+}, R_{i}^{+}\right)+O_{i j}^{L} \Delta C\left(T_{i}^{+}, s_{i j}^{+}, R_{i}^{+}\right)\right\} \\
& +K_{\nu} \xi_{L} \sum_{i=1}^{2} \frac{\left|V_{i 1}\right|^{3}}{\sin ^{2} \omega_{-i n} \sin 2 \epsilon_{-}}\left[\Delta G\left(T_{i}^{+}, R_{i}^{+}\right)+\cos 21_{-} \Delta G(T, R=0)\right] \text {. }
\end{aligned}
$$

\section{Lare Mass Limits}

Various limiting fosms of the intezral funetions are presented in section M.7 of the following appendix. We assert that (L.30) is welf-behaved in the limit that any of the masses involved or $q^{7}$ or any of the defined ratios thereof (T,S and $R$ ) becomes large. In this limit the decoupling theorem enters with full force and contributions from very heavy states become negligible. This to readily evident for most of the limits. When we take $T$ large (i.e. $m_{i}$ large) this requires a bit of work. As a reminder:

$$
T_{i}=\frac{m_{l}^{2}}{m_{x_{i}}^{2}} \quad S_{i j}=\frac{m_{\overline{x i}}^{2}}{m_{x_{i}}^{2}} \quad R_{i}=\frac{q^{2}}{4 m_{x_{i}}^{2}}
$$




$$
\begin{aligned}
& \lim _{J \rightarrow \infty} I_{\{\mathbb{N}(2,2)\}}(T, S, R) \rightarrow 0 \\
& \lim _{J \rightarrow \infty} \tilde{G}(T, S, R) \rightarrow 0 \\
& \lim _{J \rightarrow \infty} G(T, S, R)--\mid \ln T \\
& \lim _{T \rightarrow \infty} \mathcal{G}(T, S, R) \rightarrow-\mid \ln T
\end{aligned}
$$

Suppose that we consider what happens if $m_{\xi_{1}}, m_{n_{2}} \rightarrow \infty$. We find that

$$
\Delta G, \Delta G \rightarrow-\ln \frac{m_{i_{1}}^{2}}{m_{i_{1}}^{2}}
$$

and the charging matrix element becarats

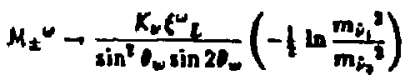

$$
\begin{aligned}
& \times\left\{\sum_{i=1}^{2}\left|v_{i j}\right|^{4}\left(1+\cos 20_{w}\right)+2 \sum_{i j=1}^{3} v_{j 2} V_{i 2} \cdot O_{j j}^{\prime}\right\}
\end{aligned}
$$

and we recall that

$$
O_{i j}^{L}{ }^{\prime}=\delta_{i j} \sin ^{2} \omega_{\omega}-v_{i 1} V_{j i}-\mid v_{i z} V_{j z}
$$

and that $V$ is unitary so that $\left(V^{\prime} V\right)_{i j}=\phi_{i j}$. Using these it is simple to show that

$$
\begin{aligned}
\sum_{i=1}^{2}\left|V_{i i}\right|^{2}\left(1+\cos 2 \theta_{w 1}\right) & =1+\cos 2 \theta_{-} \\
\sum_{i j=1}^{2} V_{j 1} V_{i i} O_{i j}^{L^{\prime}} & =\sin ^{2} \theta_{-\infty}-1
\end{aligned}
$$

273 and co

$$
\begin{aligned}
& \mu_{x}{ }^{N} \rightarrow \frac{K_{2} f_{L}}{\sin ^{2} \theta_{0} \sin 2 l_{0}}\left(-f \ln \frac{m_{E_{1}}^{2}}{m_{n_{2}}^{2}}\right) \\
& \times\left\{\left(1+\cos 2 \omega_{0}\right)+2\left(1-\sin ^{2} \theta_{0}\right)\right\} \\
& =0
\end{aligned}
$$

Therefore $\mu_{ \pm} \rightarrow 0=m_{i_{1, x}} \rightarrow \infty$.

L.2 Neutralumo Dingkaks

The neutralises contribution e may be obtained from those of the chatfinos by mating appropriate substitution. There se two enctorn to consider. The kef and sight sleptons behave somewhat differently. Rather than present all of the crossing relations, which may be obtained by examining the Feynman rules of the previous appendix, we will singly present the final result.

$$
\mu^{\nu}=M_{L} \epsilon_{L}^{\omega}+\mu_{R} \epsilon_{R}
$$

$$
f_{L, R}^{\nu}=\pi^{m}\left(p_{1}^{\prime}\right) \eta_{\tau} \tau^{\nu} \tau_{ \pm} \nu^{\prime}\left(p_{2}^{\prime}\right)
$$




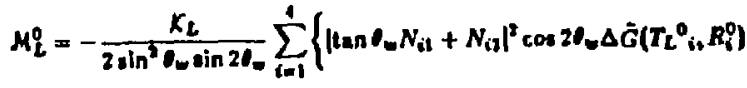

$$
\begin{aligned}
& -2 \sum_{j=1}^{1}\left(\tan D_{\infty} N^{\cdot}{ }_{i 1}+N{ }^{\cdot}{ }_{i 2}\right)\left(\tan \theta_{\infty} N_{j 1}+N_{j 8}\right) \\
& {\left[\sqrt{S_{i j}^{0}} o^{n_{i j}^{\prime \prime}} \Delta J_{[1]}\left(T_{L}{ }^{0}{ }_{i,} s_{i j}^{0}, R_{i}^{0}\right)\right.} \\
& -R_{i}^{0} O^{L \prime \prime} \Delta I_{\left[\tilde{z}^{3}-z^{2}\right]}\left(T_{L}{ }^{0}{ }_{i,} S_{i j}^{0}, R_{i}^{0}\right) \\
& \left.\left.+O^{L \prime \prime} \Delta \sigma^{2}\left(T_{L}{ }^{0}, s_{i j}^{0}, R_{i}^{0}\right)\right]\right\}
\end{aligned}
$$

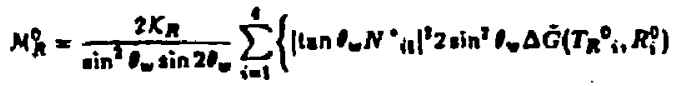

$$
\begin{aligned}
& +2 \sum_{j=1}^{1}\left(\tan \theta_{w} N_{i 1}\right)\left(\tan \theta_{0,} N^{\circ} j\right) \\
& {\left[\sqrt{S_{i j}^{0}} O^{2 ! i j} \Delta\right]_{(1)}\left(T_{R}^{0}{ }_{i}, S_{i j}^{0}, R_{j}^{0}\right)}
\end{aligned}
$$

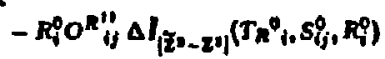

$$
\begin{aligned}
& \left.\left.+O^{R \prime \prime} \Delta G\left(T_{R}^{0}{ }_{i}, S_{i j}^{0}, R_{i}^{0}\right)\right]\right\} \\
& x_{\nu}=\frac{i}{16 \pi^{2}} e^{3} \sin \theta_{v} \cos \theta_{\nu} \\
& X_{L}=\frac{i}{16 \pi^{2}} e^{3} \sin O_{L} \cos O_{L} \\
& X_{R}=\frac{i}{16 \pi} e^{3} \sin t_{R} \cos \theta_{R}
\end{aligned}
$$

$R_{1} S_{1}$ and $T$ are ratios of masses:

$$
T_{L, R_{1}^{0}}=\frac{m_{L_{L, R_{1}}^{3}}}{m_{\dot{x} l^{2}}}
$$

$$
\begin{aligned}
& S_{i j}^{0}=\frac{m_{\dot{x}_{i}^{2}}}{m_{\dot{x}_{i}^{2}}^{2}} \\
& R_{i}^{0}=\frac{\ell^{\prime}}{4 m_{\dot{x}_{i}^{2}}}
\end{aligned}
$$

where $m_{\tilde{i}_{L_{1}}}$ and $m_{\bar{R}_{n_{1}}}$ are the alepton mussen.

$A$ typical substition would involve going from the $r-\bar{n}_{i} \bar{x}_{j}^{+}$to the $r-\bar{i}_{n_{1}} \dot{x}_{j}^{0}$ vertex. Wie would meke the cubutitutions

$$
-i g V_{j 1} \rightarrow-i \sqrt{2} g \tan 0_{2} N_{j i} \quad T_{+} \rightarrow \gamma_{-} \quad \theta_{n} \rightarrow \theta_{k} .
$$

\section{L.3 Complete Matrdx Element}

Summarizing, the complete matrix element is the total of the charaino and neutrallino contributlons. It ta glven by

$$
M^{L}=M_{2} E_{L}{ }_{L}+M_{0} \xi^{L}{ }_{R}
$$

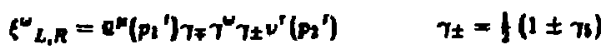

$$
\begin{aligned}
& K_{\nu}=\frac{i}{16 \pi^{2}} e^{3} \sin \theta_{\nu} \cos \theta_{\nu} \\
& X_{L}=\frac{i}{16 \pi^{2}} e^{3} \sin \theta_{L} \cos t_{L} \\
& X_{n}=\frac{i}{16 \pi^{2}} e^{3} \sin 0_{R} \cos \theta_{R}
\end{aligned}
$$




$$
\begin{aligned}
& M_{L}=\frac{X_{\nu}}{\sin ^{2} \omega_{\omega} \sin 2 \ell_{w}} \sum_{i=1}^{2}\left\{\left|V_{i 1}\right|^{2}\left[\Delta G\left(T_{i}^{+}, R_{i}^{+}\right)+\cos 2 Q_{w} \Delta G\left(T_{i}^{+}, 0\right)\right\}\right. \\
& +2 \sum_{j=1}^{2} V_{i j} V_{j 1}\left[\sqrt{S_{i j}^{+}} O_{j j}{ }^{\prime} \Delta\right\rfloor_{j !}\left(T_{j}^{+}, S_{i j}^{+}, R_{i j}^{+}\right] \\
& -R_{i}^{+} O_{i j}^{[\prime} \Delta J_{[\tilde{z}=-z y]}\left(T_{i}^{+}, s_{i j}^{+}, R_{i}^{+}\right) \\
& \left.\left.+O_{i j}^{L \prime} \Delta G\left(T_{i}^{+}, S_{i j}^{+}, R_{i}^{+}\right)\right]\right\}
\end{aligned}
$$

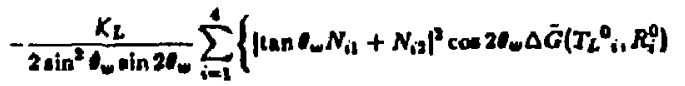

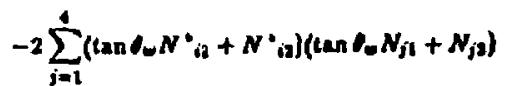

$$
\begin{aligned}
& {\left[\sqrt{S_{i j}^{0}} O^{n ! \prime \prime} \Delta I_{(1)}\left(T_{l}^{0}{ }_{i,} S_{i j}^{0}, S_{i}^{0}\right)\right.} \\
& -R_{i}^{0} O^{L i,} \Delta J_{\left[\tilde{z}^{*}-20 \mid\right.}\left(T_{L}^{0}{ }_{i}, S_{i j}^{0}, R_{i}^{0}\right\} \\
& \left.\left.+O^{L i j} \Delta G\left[T_{L}{ }^{0}{ }_{i}, S_{i,}^{0}, B_{i}^{0}\right)\right]\right\}
\end{aligned}
$$

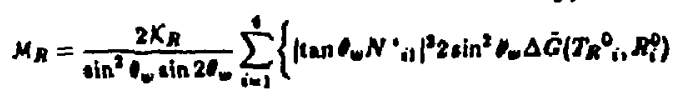

$$
\begin{aligned}
& +2 \sum_{j=1}^{1}\left(\tan \theta_{w} N_{i 1}\right)\left(\tan \theta_{-\infty} N^{\circ}{ }_{j 1}\right) \\
& {\left[\sqrt{S_{i j}^{0}} O_{i j}^{L, 1 "} \Delta_{[\mathrm{p}]}\left(T_{R}^{0}{ }_{i,} S_{i j}^{0}, R_{i}^{0}\right)\right.} \\
& -R_{i}^{0} O^{R \prime \prime} \Delta I_{\left[\tilde{z}^{2}-22\right\}}\left(T_{R^{0}}^{0}, S_{i j}^{0}, R_{i}^{0}\right) \\
& \left.\left.+O^{R}{ }_{i j}^{\prime \prime} \Delta G\left(T_{R}{ }_{i}, S_{i j}^{Q}, R_{i}^{Q}\right)\right]\right\}
\end{aligned}
$$

\section{$R, S$, end $T$ are ration of mantes:}

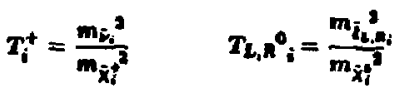

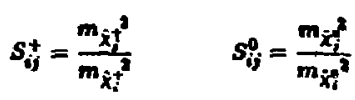

$$
\begin{aligned}
& R_{i}^{+}=\frac{e^{2}}{4 m_{i_{i}^{2}}^{2}} \quad \cdot \quad R_{i}^{0}=\frac{\varphi^{2}}{1 m_{x_{i}^{0}}^{2}}
\end{aligned}
$$

where $m_{\tilde{f}_{t_{1}}}$ and $m_{\tilde{i}_{n_{1}}}$ are the meses of the charged skpton of the Brat generation mixture (we eqn. 3a) and $m_{n}$ in the mass of the first sneutrino $\left(\tilde{V}_{1}=\tilde{v}_{\mu} \cos \theta_{\mu}+\right.$

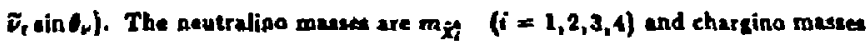
are $m_{\dot{x}_{i}}(i=1,2)$. 


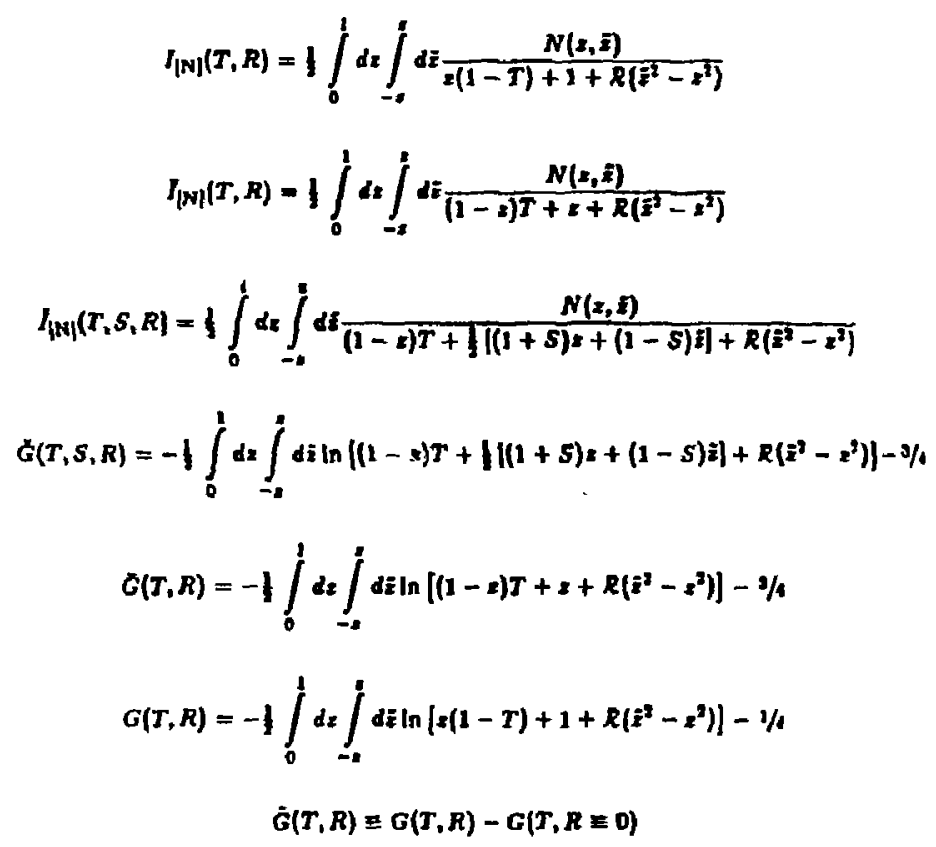

Note that

$$
\begin{aligned}
& G(T, R)=G(T, S=1, R) \\
& I_{|N|}(T, R)=I_{|N|}(T, S=1, R) \\
& I_{|N|}(T, R)=\frac{1}{T} I_{|N|}\left(\frac{1}{T}, \frac{R}{T}\right) \\
& I_{[N \mid}(T, R)=\frac{1}{T} I_{|N|}\left(\frac{1}{T}, \frac{R}{T}\right) \\
& G(T, R)=G\left(\frac{1}{T}, \frac{R}{T}\right)+f \ln T \\
& I_{(R P}(T, R)=\frac{1}{2 R} \bar{G}(T, R) \\
& \text { For any function, } F, \text { of } T_{i}^{0,+} L_{t, R} \\
& \left.\Delta F \equiv F\right|_{i_{1}, 0_{0}}-\left.F\right|_{i_{1}, b_{1}}
\end{aligned}
$$




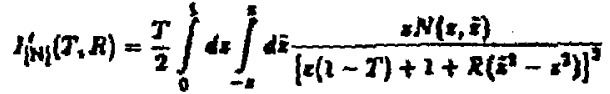

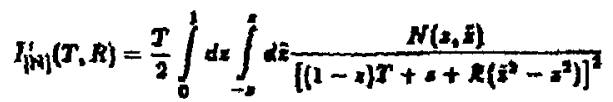

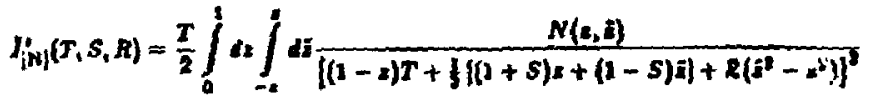

$\sigma^{\prime}(T, S, R) \approx-T I_{[1-1]}(T, S, R)$

$G^{\prime}(T, R)=-T I_{(1-1)}^{\prime}(T, R)$

$G^{\prime}(T, R)=-T X_{(1-g)}(T, R)$

$\hat{G}\left\{(T, R) \approx-T\left[H_{1,1}(T, R)-H_{\mu, 1}(T, R \approx 0)\right]\right.$

$$
G^{\prime}(T, R)=1-G^{\prime}\left(\frac{2}{T}, \frac{R}{T}\right)
$$

$L_{(a)}(T, R=0)=\frac{2 T \ln T+1-T^{2}}{(T-J)^{2}}$
1. Disgram One.

3. Diagram Two.

3. Dineram Thres.

4. Diagram Four. 

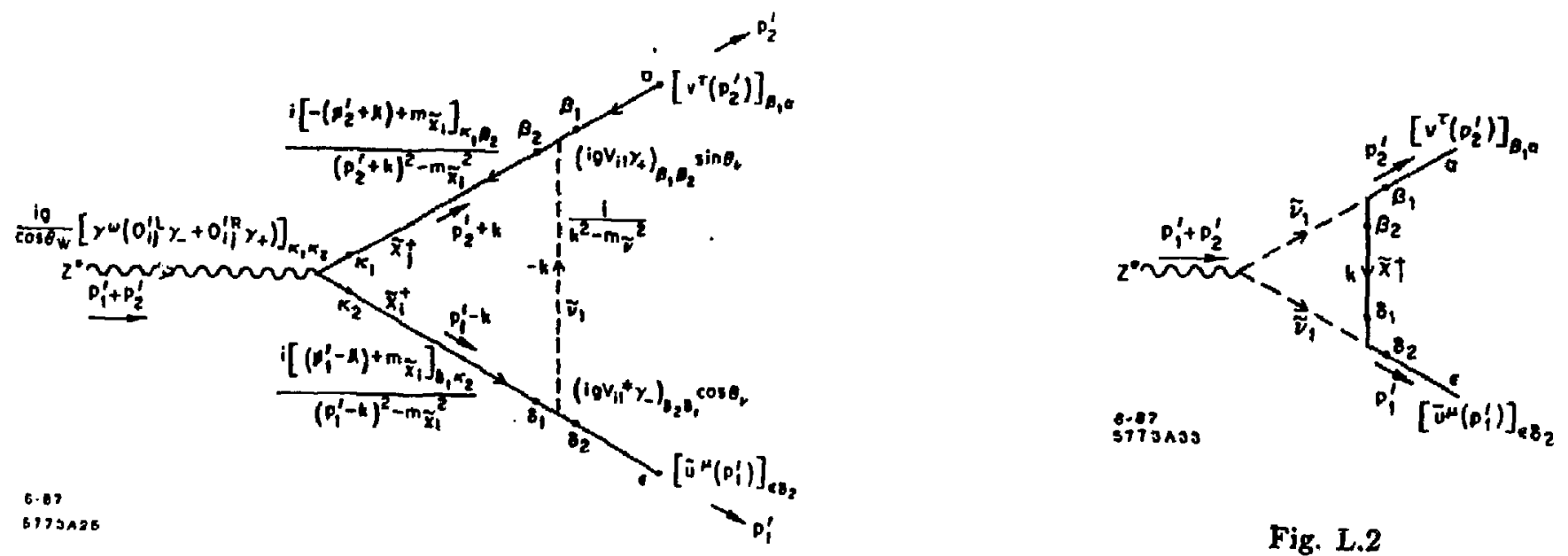

Fig. L.1

Fig. L.2 


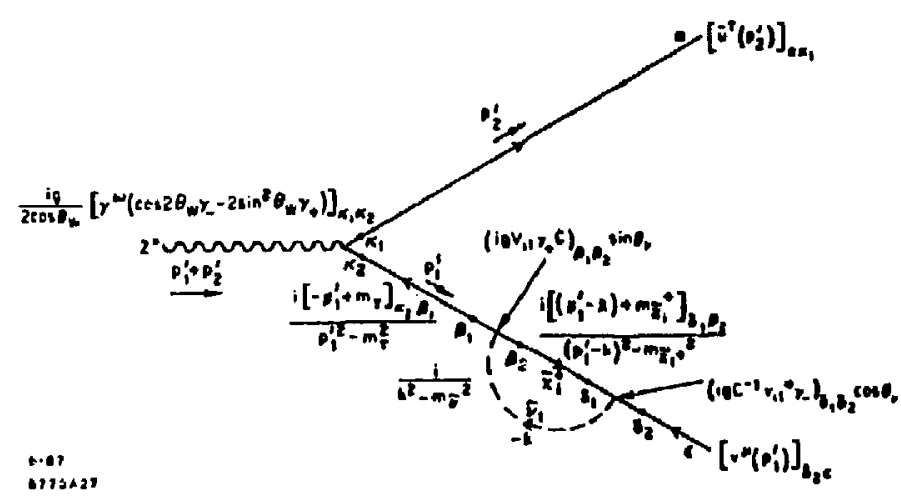

Fig. L.3

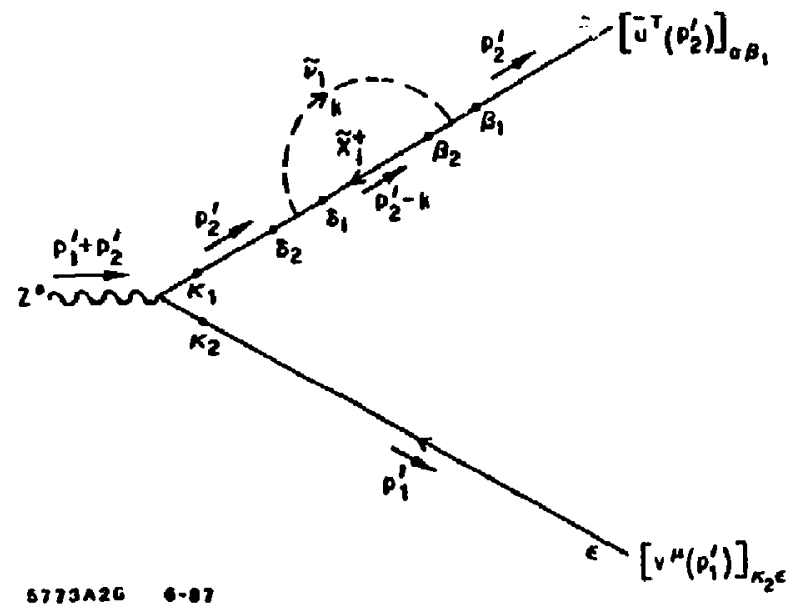

Fig. L.4 


\section{APPENDIX M}

Analysis of Integral Functions

M.I Javagunt Fona or I and $G$ Inteokals: $S_{i j}$ versus $S_{j i}$

We know lrom physical intuition that when we inlerchange $\tilde{x}_{i}$ and $\tilde{x}_{j}$ in the vertex shown in Fig. I that thls should not afiect our answer.

Here $\bar{x}_{i}$ is $\tilde{x}_{i}^{+, 0}, \quad m_{\tilde{i}}=m_{\tilde{i}_{i n}}$ ate. Uainc these abrevialed forms we may lso shorten the mass ratios oricinally defined in $64 n, 7.10$ to

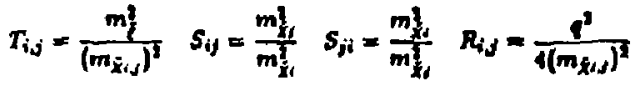

$$
\begin{aligned}
& s_{i j}=\frac{1}{s_{j}}
\end{aligned}
$$

Then

$$
\begin{aligned}
& I_{(\mathrm{N})}\left(T_{i}, S_{i j}, R_{i}\right)=1 \int_{0}^{1} d x \int_{-=}^{l} d i
\end{aligned}
$$

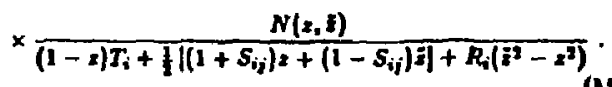

Now multiply by $s_{j d} / s_{j i}$ to use (II.2)

$$
\begin{aligned}
& T_{[N]}\left(T_{i}, s_{i j}, R_{i}\right)
\end{aligned}
$$

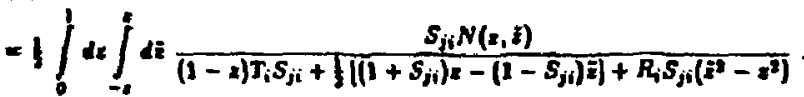

Now

$$
T_{i} S_{j i}=T_{j} \text { and } R_{i} S_{j i}=R_{j} \text {. }
$$

If $N(z, \hat{z})=N(z,-\hat{x})$ we let $\bar{z} \rightarrow-\bar{z}$ in $(M, 4)$ and using (M.5) get

$$
\begin{aligned}
& \left.I_{[N \mid}\left(T_{i}, S_{i j}, R_{i}\right)=\left(S_{j i}\right)\right) \int_{-2}^{1} d z \\
& \times \frac{N(z, E)}{(1-s) T_{j}+\left[\mid\left(1+S_{j i}\right)+\left(1-S_{j i}\right) z^{2}\right]+R_{i}\left(z^{2}-s^{2}\right)} \\
& =S_{j i} f_{[N]}\left(T_{i,} S_{j i}, R_{j}\right)
\end{aligned}
$$

or

$$
\sqrt{s_{i j}} I_{\{\mathbb{N}\}}\left(T_{i}, s_{i j}, R_{i}\right)=\sqrt{s_{j i}} I_{(\mathbb{N})}\left(T_{j}, s_{j i 1} R_{j}\right) .
$$

Similstly from (M.s) and (M.6)

$$
\begin{aligned}
& T_{i} I_{(N) \mid}\left(T_{i}, S_{i j}, R_{i}\right)=T_{j} I_{(N)}\left(T_{i}, S_{j i}, R_{i}\right) \\
& R_{i} I_{(N)}\left(T_{i}, S_{i j}, R_{j}\right)=R_{j} I_{(N)}\left(T_{j}, S_{i j}, R_{j}\right)
\end{aligned}
$$

In particula, of the functions which appear in (3.9), $\sqrt{S} \mathbf{I}_{|\eta|}$ and $\left.R\right|_{\left|I^{\prime}-x^{\prime}\right|}$

$$
\sum_{i j} \sqrt{s} I_{[1]}=2 \sum_{-i, 3-1,} \sqrt{s} I_{\mid 1\}}+\sum_{i} I_{11}
$$

The lalter term being the $i=j$ term vince $I=I$ with $S=1$. Likewise

$$
\begin{aligned}
& \sum_{i j} R_{i} l_{\left[i^{*}-i\right]}\left(T_{i}, S_{i j}, R_{i}\right)
\end{aligned}
$$

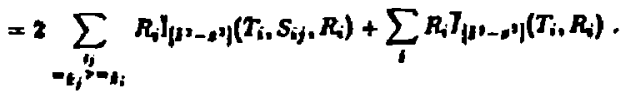


The sum:

can also be written

$$
\sum_{\substack{i, j \\-i, j,-i_{i}}}
$$

$$
\sum_{\substack{j \\ \text { dit }}}
$$

A similar result holds for the $G$ functiona. From Table L.I, betting $E \rightarrow-i$ U $N(x, \bar{z})$ is even in $\bar{z}$, yields

$$
G\left(T_{i}, S_{i j}, R_{i}\right)=G\left(T_{j}, S_{j i}, R_{j}\right)+\mid \ln S_{j i}
$$

and the "invariant" form in

$$
G\left(T_{i}, S_{i j}, R_{i}\right)+\frac{1}{4} \ln S_{i j}=G\left(T_{j}, S_{j i}, R_{j}\right)+\frac{1}{4} \ln S_{j i}
$$

and similarly

$$
\begin{aligned}
& G\left(T_{i}, S_{i j}, R_{i}\right)+\left|\ln R_{i}=G\left(T_{j}, S_{j i}, R_{j}\right)+\right| \ln R_{j} \\
& G\left(T_{i}, S_{i j}, R_{i}\right)+i \ln T_{i}=G\left(T_{j}, S_{j i}, R_{j}\right)+\left\lfloor\ln T_{j} .\right.
\end{aligned}
$$

Fram Table L.

$$
G^{\prime}\left(T_{i}, S_{i j}, R_{i}\right)=-T_{i} J_{1--1}\left(T_{i}, S_{i j}, R_{i}\right)
$$

so that

$$
G^{\prime}\left(T_{i}, S_{i j}, R_{i}\right)=G^{\prime}\left(T_{j}, S_{j i}, R_{j}\right) .
$$

Thus in all of the ter ms in $\mathrm{M}^{4}$, both large and small maxs-splitting cases considered, we may restrict our altention to the $S_{j i} \geq 1$ contributions. This will prove convenient fot numerical evaluation.

\section{M.2 In(z,2) Integrals: Imaginany Part}

\section{Same fun with camplex analysi:}

The propugator for the ith boson [fermion] 1 .

$$
\Delta_{\text {ipropesules }}=\frac{i\left(\left(f_{1}+m_{i}\right)\right]}{p_{i}^{2}-m_{i}^{2}+i c_{i}}
$$

so we just abeorb the it into the $m^{2}$ term

$$
m_{i}^{2} \rightarrow m_{i}^{2}-i i_{i}
$$

The $\mathrm{I}_{\mathrm{iN}}$ lerons came from diagrame like thoet of Fiz. 2 involving loops of three propagators, ench with their own, orbitrary ic. Frow eqn. (L.J) we are kead to integralk like

$$
2 \sim \int_{0}^{1} d x
$$

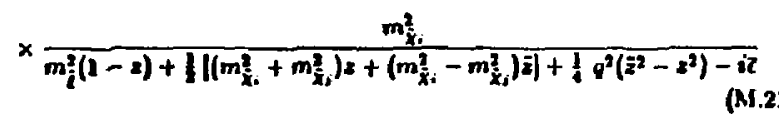

where

$$
I=I_{i}(1-x)+\left\{\mid\left(z_{x_{i}}+z_{x_{j}}\right) z+\left(z_{x_{i}}-z_{x_{j}}\right) i\right] .
$$

Since each $c_{j}$ is of arbitrary relative magnitude the overall sign of $t$ might appear to be parameter-dependent in a way which could render the relatiuc signi of different contributions ambigous. Our task is to prove that this is not the case and to derive the correct sign. 
Divide through by $m_{1}^{2}$

$$
I \sim \int_{0}^{l} d x \int_{-=}^{l} d i \frac{1}{T_{i}(1-z)+\left[\left(1+S_{i j}\right) \pm+\left(1-S_{i j}\right)\right]+R_{i}\left(i^{4}-z^{2}\right)-i e}
$$

(M.23)

where

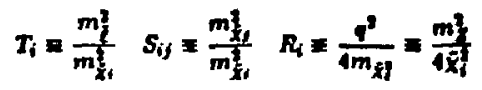

here (a in (M.1)). We re-cebine

$$
=\frac{1}{m_{x}^{2}}
$$

and, in general,

$$
c_{j}=\frac{1}{m_{k_{1}}^{2}}
$$

(i subscript understood).

The slepton oases, $m_{i}$, as e $\left(m_{i_{L_{i}}}, m_{i_{n_{i}}}, m_{z_{1}}\right)$ and $m_{k_{1}}$ is it gaugino/hizatino mass. We can choose $S_{i j} \geq 1$ as discussed above and so we have

$g^{2}=m_{i}^{2}>0 \quad \pi_{1} \geq 0 \quad R_{i} \geq 0 \quad S_{i j} \geq 1 \quad 0 \leq \leq \leq 1 \quad-s \leq \leq \leq$

and all $c>0$.

(M.26)

Now look at the most general such integral of the type in (M.23). Pulling " $R_{i}$ " out of (M.23) we may write (making the expression exact)

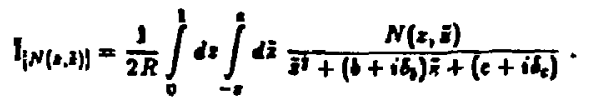

(M.27)
Comparing with (M.23), (M.24), and (M.25)

$$
\begin{aligned}
& b=\frac{1-s}{2 R}<0 \\
& \delta_{i}=-\frac{2}{t^{2}}\left(\mathrm{E}_{x_{i}}-e_{x_{i}}\right)
\end{aligned}
$$

which is of no fixed sign since $t_{j}, t_{j}$ are arbitrary and independent

$$
\begin{aligned}
& c=-\left[z^{2}-\frac{T(1-z)+\mid(1+s) x}{R}\right] \\
& c_{t}=-\frac{1}{h^{2}}\left|c_{i}(1-z)+\right|\left(\mathrm{f}_{x_{1}}+\left.\right|_{x_{1}}\right) s \mid<0
\end{aligned}
$$

(M.20)

where the inequality follows from (M.26) vince all the bracketed terms are post-

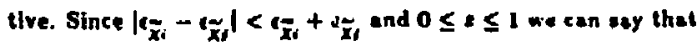

$$
0<2\left|\delta_{0}\right|<-8,
$$

(M.30)

For (M.27) write

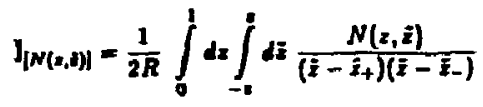

where

$$
\left.z_{ \pm} \doteq\right\rfloor\left[-\left(b+i \delta_{b}\right) \pm \sqrt{b^{3}+2 i b \delta_{t}-i c-1 \sigma_{c}}\right]
$$

Letting

$$
\Delta=b^{2}-4 c \pm 40=4\left[\left(x+\frac{1}{2}\right)^{2}-\frac{(1-z) T+2}{R}\right]
$$

defines $Q$ as

$$
Q \mp\left(z+\frac{b}{2}\right)^{2}-\frac{(1-2) T+z}{R} \geq 0
$$

since $\Delta \geq 0$ or else there is no imaginary part. This follows once $\Delta$ is, essentially

291

292 
the diecriminant of the denominator of (M.22). If $\Delta<0$ then the denominator hes no real roole and is always posilive or negative definlte over the range of integration. The result is real integral. Note that since $(1-2), 2, T$ and $R \geq 0$

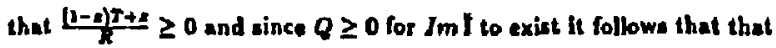

$$
Q<(x+b / 2)^{2}
$$

Defan:

$$
\begin{aligned}
r_{ \pm} & =R e\left|\hat{z}_{ \pm}\right| \\
& =-\frac{6}{2} \pm \sqrt{9}
\end{aligned}
$$

$80 r+>r_{-}(Q \geq 0)$

$$
i_{ \pm}=r_{ \pm}+j i\left[c_{t} \pm i \frac{\left(\omega_{6}-2 t_{6}\right)}{2 \sqrt{9}}\right]
$$

Writing

$$
i_{ \pm}(z)=r_{ \pm}(s)+i_{ \pm}(z)
$$

our analysis will reduce to determining the signs of $C_{ \pm}$.

$$
\begin{aligned}
\epsilon_{ \pm} & =-\left[\delta_{b} \mp \frac{b \delta_{b}}{2 \sqrt{Q}} \pm \frac{2 \delta_{c}}{2 \sqrt{Q}}\right]=-\frac{1}{2 \sqrt{Q}}\left[\delta_{b}\left(\sqrt{Q} \mp \frac{b}{2}\right) \pm \delta_{c}\right] \\
& \left.=\mp \frac{1}{2 \sqrt{Q}} \mid r_{ \pm} \delta_{b}+\delta_{c}\right]
\end{aligned}
$$

Define

$$
F_{*}=-\frac{1}{2} \pm \sqrt{\frac{(1-2) T+2}{R}}
$$

and nole that

$$
F_{+}>F_{-} \cdot
$$

Then

$$
Q=\left(z+\frac{b}{2}\right)^{2}-\frac{(1-r) T+z}{R}=\left(x-T_{+}\right)\left(z-r_{-}\right)
$$

Of course from (M.35)

$$
\left(x-F_{+}\right)\left(x-F_{-}\right)=Q>0 .
$$

So the cases are

$$
\begin{aligned}
& \text { (i) } z-r_{+}>0 \text { and } x-r_{-}>0 \\
& \text { (ii) } x-r_{+}<0 \text { and } x-r_{-}<0
\end{aligned}
$$

Litewise

$$
\left(z-r_{+}\right)\left(z-r_{-}\right)=\frac{(1-x) T+z}{B}>0
$$

Cases

(i) $z-r_{+}>0$ and $z-r_{-}>0$

(ii) $z-r_{+}<0$ and $x-r_{-}<0$

and in all cases $0 \leq z \leq 1$. From (M.31) and (M.38)

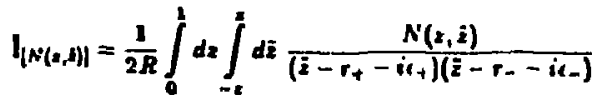

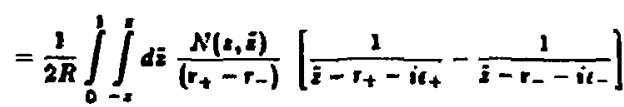


with

$$
r_{ \pm}=-\frac{1}{2} \pm \sqrt{Q}
$$

and therefore

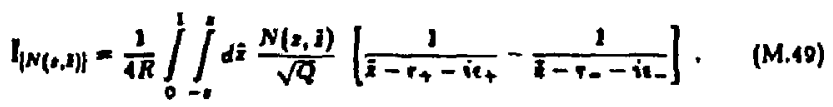

Principal Value Formula

Under $\int d x$ :

$$
\frac{1}{x-a \pm i e} \rightarrow \frac{1}{x-a} \mp i x 6(x-a)
$$

$$
\begin{aligned}
& \operatorname{Im} I_{[N(n, J)]}=\frac{i \pi}{s R} \int_{0}^{1} \int_{-\pi} d t \\
& \times \frac{\varphi(Q)}{\sqrt{Q}} N\left(z_{i} i\right)\left\{\operatorname{sign}\left(e_{+}\right) 6\left(i-t_{+}\right)-\operatorname{aign}\left(e_{-}\right) 6\left(\bar{z}-r_{-}\right)\right\}
\end{aligned}
$$

since $Q>0$ for an In part to exist. The aisnum function, sign $(X)$, is defined to be :

$$
\begin{aligned}
& \operatorname{sizn}(x)= \begin{cases}-1 & x<0 \\
0 & x=0 \\
2 & x>0\end{cases} \\
& I m y_{\left\{N\left(r_{1}, t\right)\right\}}=\frac{i \pi}{i R} \int_{0}^{1} d u \frac{Q(Q)}{\sqrt{Q}} \mid \operatorname{sicn}\left(c_{+}\right) N\left(x_{i} r_{+}\right)\left(Q\left(z-r_{+}\right)-\theta\left(-z-r_{+}\right)\right\} \\
& \left.-\sin \left(u_{-}\right) N\left(x, r_{-}\right)\left(0\left(x-r_{-}\right)-(1-x-r-)\right]\right]
\end{aligned}
$$

(M.53)
Now from (M.35)

$$
\sqrt{Q}<\left|c+\frac{b}{2}\right| \leq\left|s-\frac{b}{2}\right| \quad(x \geq 0 \quad 0 \leq 0)
$$

(M.s4)

and once

$$
=-\frac{1}{2}>0 \quad \sqrt{9}<2-\frac{6}{2}
$$

we ind

$$
-\left(x-\frac{b}{2}\right) \pm \sqrt{a}<0
$$

(M.56)

and therefore

$$
(-2-17)=1\left(-2+\frac{1}{2} \mp \sqrt{Q}\right)=0\left(-\left[z-\frac{1}{2}\right] \mp \sqrt{Q}\right)=0
$$

with the result that (M.S3) reduces to

$$
\begin{aligned}
& \operatorname{Im} I_{[N(x, 1)]}=\frac{i \pi}{4 R} \int_{0}^{1} d x \\
& \frac{\varphi Q)}{\sqrt{Q}}\left(N\left(x_{1} r_{+}\right) \operatorname{sign}\left(e_{+}\right) U\left(x-r_{+}\right)-N\left(x_{1} r_{-}\right) \operatorname{sign}\left(x_{-}\right) U\left(x-r_{-}\right)\right) \text {. }
\end{aligned}
$$

(M.sB)

Since the two terms are proportional to $\varphi\left(z-r_{+}\right)$and $\varphi\left(z-r_{-}\right)$we require $2-r_{+}$ and/or * - r - to be positive in order to gel a contribution. Looking at (M.16) and (AS.47) we see that this rules out case (ii).

We must have

$$
z-x_{ \pm}>0
$$

This can be understood in the following whey:

975

29: 
Since $\frac{(1-g) T+2}{k}>0$ we could write a factor of

$$
\text { - }\left(\frac{(1-x) T+x}{R}\right)=1
$$

(M.60)

in $\mathrm{I}_{[N]}$ just like $O(Q)$

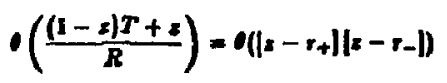

(M.61)

80 fram (M.sB)

$$
\begin{aligned}
& \operatorname{Im} I_{[N(a, B)]}=\frac{i \pi}{A R} \int_{0}^{l} d x \frac{O(Q)}{\sqrt{Q}} \cdot(\mid x-r+\|x-r-\|) \\
& \left.\left.\times \|\left(z-r_{+}\right) \operatorname{rign}\left(\ell_{+}\right) N\left(z_{2}, r_{+}\right)-O_{2}-r_{-}\right) \operatorname{sign}\left(C_{-}\right) N\left(z, r_{-}\right)\right]
\end{aligned}
$$

(A1.62)

bus

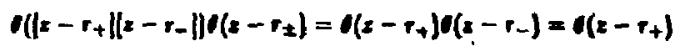

(M.63)

since $r_{+}>r_{-}$, i.e.

$$
0<r_{+}<2 \leq 1 \text { also } r_{-}<r_{+}<2 \leq 1
$$

(M.61)

the net sesult being

$$
\left.I m\right|_{i N(n, i)]}=\frac{i \pi}{d R} \int_{0}^{1} d z \frac{(Q) Q\left(z-r_{1}\right)}{\sqrt{Q}}\left|\operatorname{sign}\left(c_{+}\right) N\left(z, r_{+}\right)-\operatorname{sign}\left(c_{-}\right) N\left(z, r_{-}\right)\right| .
$$

( $(\mathbf{1 . 6 5 )}$
From (M.s7) $-x-P-<0$ and thesefore $-<<-$. With this and from (M.en)

$1 \leq-2<r_{-}<r_{+}<x \leq 1$ with $0<r_{+}<x \leq 1$

(M.66)

Fromo (M.36) and (AS.40)

$$
\begin{aligned}
& F_{+}-t_{-}=\sqrt{\frac{(1-g) T+z}{A}}+\sqrt{Q}>0 \\
& r_{+}-T_{-}=\sqrt{\frac{(1-\Omega) T+x}{R}}+\sqrt{Q}>0
\end{aligned}
$$

(M1.67)

$\omega$

$F_{-}<r_{+}<x \leq 1$

(M.68)

and henee

$$
x-7 . .>0
$$

(M1.69)

From (M.43) and (M.14) case (ii) has juat been eliminaled lesding to the inescapable reault that

$$
=-z_{ \pm}>0
$$

Thus (bec ause r+ $>r_{-}$)

$$
\varphi(Q)=\varphi\left(\left(x-F_{+} \| z-F_{-}\right)\right)=\varphi\left(z-F_{+}\right) O\left(z-F_{-}\right)=\varphi\left(z-F_{+}\right) .
$$

(M.71)

Also

$$
F_{+}-x<0 \Rightarrow-z-\frac{b}{2}+\sqrt{\frac{(1-2) T+8}{R}}<0 .
$$

Now

$$
-z-i_{-}=-z+\frac{b}{2}+\sqrt{\frac{(1-2) T+z}{R}}<-z-\frac{b}{2}+\sqrt{\frac{(1-z) T+z}{R}}<0
$$

from (M. .72) since $b<0$.

297

208 
Therefore

r. $>-2$

(M.74)

Also note that from (M.72)

$$
\begin{gathered}
-1 \leq-1<r-,+r, r_{+}<2 \leq 1 \quad r+>0 \\
0<\sqrt{\frac{(1-1) T+2}{R}}<2+\frac{b}{2}
\end{gathered}
$$

i.e.

$$
2+\frac{1}{2}>0
$$

so usine (M.71) and (M.65)

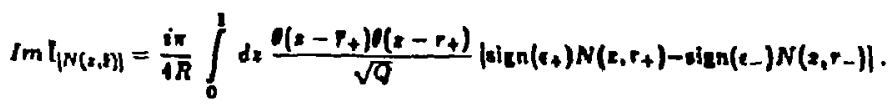

Note that

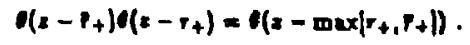

Frow (M.75)

$$
|r \pm|<*
$$

Resall from (M.30) and (M.39) that

$$
c_{ \pm}=\mp \frac{1}{2 \sqrt{Q}}\left|r_{ \pm} \delta_{b}+\delta_{c}\right| \text { and } s\left|\delta_{b}\right|<\delta_{c}<0 \text {. }
$$

Since

$$
|r-1<x \Rightarrow| r_{*} \delta_{3}|<x| \delta_{3}|<| \delta_{1} \mid
$$

we see that

$$
\begin{aligned}
& \operatorname{sicn}\left|r_{*} \delta_{t}+\delta_{c}\right|=\operatorname{sign}\left|\delta_{c}\right|=-1 \\
& \operatorname{sign}\left(c_{ \pm}\right)= \pm 1
\end{aligned}
$$

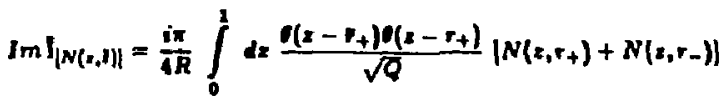

where we recall that

$$
\begin{aligned}
& y_{ \pm}=-\frac{b}{2} \pm \sqrt{\frac{(1-z) T+z}{R}} \\
& r_{ \pm}=-\frac{i}{2} \pm \sqrt{Q} \\
& \theta=\left(z+\frac{b}{2}\right)^{2}-\frac{(1-z) T+z}{R} \\
& t=\frac{(1-s)}{2 R}
\end{aligned}
$$

$T, S$, and $R$ are $a$ in (M.24).

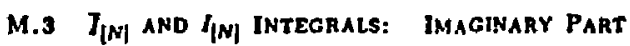

We may also obtain $I_{\{N(:, 5)\}}$ directly from $(M, 85)$ by letting $\rightarrow 1$. Then $b=0$ and

$$
P_{ \pm} \rightarrow \pm \sqrt{\frac{(1-3) T+z}{R}}
$$


$r_{ \pm} \rightarrow \sqrt{\bar{Q}}$

(Asses)

with

$$
\bar{Q}=z^{2}-\frac{(1-x) T+2}{\Omega}
$$

(AS.80)

$$
\begin{aligned}
& \operatorname{Im} I_{\{N(s, b)\}} \\
& =\frac{i x}{4 R} \int_{0}^{1} d x \frac{\cdot\left(2-\sqrt{\frac{(1-1) \sqrt{x+1}}{h}}\right)(2-\sqrt{Q})}{\sqrt{Q}}[N(2, \sqrt{Q})+N(2,-\sqrt{Q})] \text {. }
\end{aligned}
$$

(M.⿻)

Note that ir $N(x, \hat{z})$ is even in $z$, ie. $N(x, \hat{z})=N(x,-\bar{z})$ the o

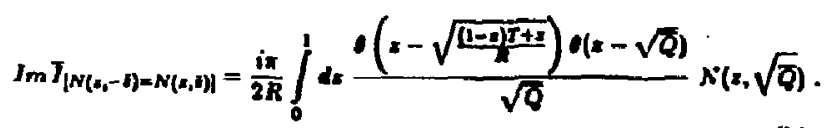

(M.91)

If $N(s, z)$ is odd in $z$, life. $N(x,-\tilde{z})=-N(x, \bar{x})$ then

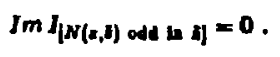

We compare $J$ and I in Table L.1.

We see that the above analysis goes through for $f_{(N)}$ if we make the changes

$$
\begin{gathered}
r_{ \pm} \rightarrow F_{ \pm}^{\prime}= \pm \sqrt{\frac{(1-2)+T_{x}}{R}} \\
r_{ \pm} \rightarrow r_{ \pm}^{\prime}= \pm \sqrt{W}
\end{gathered}
$$

(A1.03)

(M.91)

with

$$
W=x^{2}-\frac{(1-x)+I x}{R}
$$

(M.95)
Therefore

$$
\operatorname{Im} I_{|N|}=\frac{i \pi}{i R} \int_{0}^{1} d x \frac{\cdot\left(z-\frac{\left.(1-3)+r_{t}\right)}{d}\right)(z-\sqrt{W})}{\sqrt{W}}\{N(z, \sqrt{W})+N(z,-\sqrt{W})] .
$$

(M.06)

If $N(z, \bar{z})=N(z,-\bar{z})$ in even in $\bar{i}$

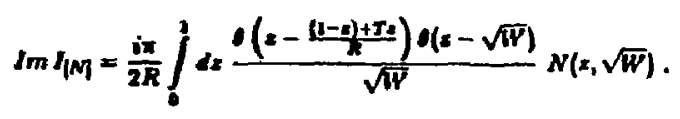

(M.97)

If $N(\boldsymbol{s}, \bar{z})=N(\boldsymbol{z},-\bar{n})$ is add in $\bar{z}$

$$
\operatorname{lm} I(N)=0 .
$$

(M.98)

M.4 $I_{[N]}, J_{(N)}$ AND $I_{[N]}$ INTEGRALS: REAL PARTS

$$
\operatorname{Re} \mathcal{I}_{|N|}=\frac{1}{2 R} \int_{0}^{1} d x \int_{-\varepsilon}^{0} d \bar{z} \frac{N(z, \tilde{z})}{z^{2}-W(z)}
$$

where $\mathrm{W}$ is given by (Mi.95). We will assume for the moment that $N=N(z)$. If $N(z, \vec{z})=\bar{z}^{2}-z^{2}$ then xe express it in terms of the above (see the next section).

Then

$$
\begin{aligned}
\operatorname{Re}(N)= & \frac{1}{R} \int_{0}^{1} d x N(z) \int_{0}^{T} d \bar{z} \\
& \times\left\{\left[\frac{1}{\bar{z}-\sqrt{W}}-\frac{1}{\bar{z}+\sqrt{W}}\right] \frac{1}{2 \sqrt{W}} O(W)+\frac{1}{\bar{z}^{2}+|W(z)|} O(-W)\right\}
\end{aligned}
$$

301

902 
and from the Principal Value Theorem,

$$
\begin{aligned}
=\frac{1}{R} \int_{0}^{1} d x N(2)\left\{\frac{1}{2 \sqrt{W}} \mid \ln (i-\sqrt{W})-\ln (i+\sqrt{W})\right\} \varphi(U W) \\
\cdot \\
\left.+\frac{1}{\sqrt{-W}} \operatorname{lan}^{-1} \frac{E}{\sqrt{-W}}(-W)\right\}\left.\right|_{z=0} ^{i=x}
\end{aligned}
$$

erso

$$
\begin{aligned}
& R e I_{[N]} \\
& =\frac{1}{R} \int_{0}^{1} d x \frac{N(2)}{\sqrt{|W|}}\left\{\ln \left[\frac{(\sqrt{W}-s)}{(\sqrt{W}+z)}\right] \varphi(W)+\tan ^{-1} \frac{1}{\sqrt{-W}} \varphi(-W)\right\} .
\end{aligned}
$$

(M.100)

The procedure for $f_{[M]}$ is similar

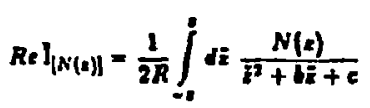

with

$$
b=\frac{1-S}{2 R} \quad c=\frac{1}{R}\left\{-R x^{2}+\frac{1+S}{2}=+(1-R) T\right\} .
$$

As in (M.33) and (M.31) let

$$
Q=\frac{1}{4}\left(b^{2}-1 c\right)=\left(2+\frac{1}{2}\right)^{2}-\frac{2(1-T)+R}{R}
$$

then

$$
\begin{aligned}
& \operatorname{Re} I_{|N|}=\frac{1}{1 A} \int_{0}^{t} \frac{N(s)}{\sqrt{|Q|}} \\
& x\left\{2 \tan ^{-1} \frac{E+b / 2}{\sqrt{-Q}}(-Q)+\left.\frac{1}{2} \ln \frac{E+b / 2-\sqrt{Q}}{z+b / 2+\sqrt{Q}}\right|_{-,} ^{*}(Q)\right\}
\end{aligned}
$$

or

$$
\begin{aligned}
& R_{e} J_{[\mathrm{N}]}=\frac{1}{2 R} \int_{0}^{1} \frac{N(x)}{\sqrt{|Q|} \mid}\left\{\left[\tan ^{-1}\left(\frac{x+4 / 2}{\sqrt{-Q}}\right)-\tan ^{-1}\left(\frac{-x+4 / 2}{\sqrt{-Q}}\right)\right] \theta(-Q)\right. \\
& \left.+\frac{1}{2} \ln \left[\frac{\left(\frac{1}{2}\right)^{2}-\left(x-\sqrt{Q)^{2}}\right.}{\left(\frac{1}{2}\right)^{2}-\left(x+\sqrt{Q)^{2}}\right.}\right]+(Q)\right\} \text {. }
\end{aligned}
$$

For $R \varepsilon \boldsymbol{T}_{|N|}$ we let $b \rightarrow 0$ in $(\mathrm{M} .104)$ above giving us back (M1.100) with "W" replaced by $\bar{Q}$ as given in (M.80).

A question arisea as to the behavior of Rel at $Q=0$. Using the eaymptotle form $(x \rightarrow \infty) \tan ^{-1} x=\pi / 2-1 / x+1 / 3 x^{3}-\ldots$ valid in the arcten sheet used

$$
\begin{aligned}
& R e l(Q \rightarrow 0) \rightarrow-\frac{1}{2 R} \int_{0}^{l} \operatorname{dx} \frac{N(\varepsilon)}{\sqrt{Q}}
\end{aligned}
$$

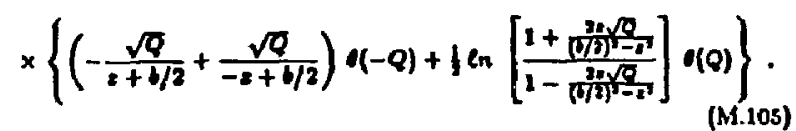

Accordingly

$$
\operatorname{Re}(Q \rightarrow 0)=\frac{1}{2 \pi} \int_{0}^{1} \mathrm{dz} N(2)\left\{\frac{2 z}{(6 / 2)^{2}-z^{2}}(Q)+\frac{2 x}{(6 / 2)^{2}-z^{3}}(-Q)\right\}(M .106)
$$

so the integrand is continuousat $Q=0$. In fact at $Q=0$ since, $\varphi(Q)+\varphi(-Q)=1$ we have

$$
\operatorname{Re} \operatorname{I}_{(N(1))}(Q=0) \rightarrow-\frac{1}{R} \int_{0}^{1} d x \frac{x N(x)}{z^{2}-(b / 2)^{2}}
$$


Note that when $b=0$ in the 7 case

$$
\operatorname{Re}]_{(N(x)\}}(\delta(x) \rightarrow 0) \rightarrow-\frac{1}{R} \int_{0}^{l} d z \frac{N(x)}{z}
$$

(M.108)

This causes no problems unless $\bar{Q}(z=0)$ happens to be zero, le. unless the pot where $\bar{\phi}$ vanishes just happens to be $s=0$. But

$$
\bar{Q}=x^{2}-\frac{(1-a) T+z}{R}
$$

(M.109)

from (A.89) 10

$$
\Phi(x=0)=\frac{T}{R}>0
$$

(M.110)

since we have taken $T, R>0$. So (M.108) b good everymbare $\left(T / R=4 \mathrm{~m} / \mathrm{s}^{2}\right.$ so there is a possible problem when $m_{j}$, the stolon mas, vanishes)

M.5 $\left.\right|_{\left.\mid \hat{x}^{*}-\mathbf{x}\right]}$

The formula for $I m I_{|N|}$ works well for $N=N(s, \bar{z})$, but (M.204) was restriated to $N=N(2)$. Recall from (M.27) that we may write

$$
\begin{aligned}
& I_{\{N \mid}(T, S, R)=\frac{1}{2 R} \int_{0}^{1} d z \int_{-1}^{\pi} d i \frac{N(z, E)}{z^{2}+b i+i \operatorname{tesm} s} \\
& b=\frac{1-S}{2 R} \quad c=\left\{-x^{2}+\left(\frac{1}{R}-b\right)=+\frac{(1-z) T}{R}\right\} .
\end{aligned}
$$

From

$$
\int d \bar{E} \frac{\bar{z}^{2}}{i^{2}+b i+c}=\bar{z}-\frac{b}{2} \ln \left(\bar{z}^{2}+b \bar{i}+c\right)+\frac{b^{2}-2 c}{2} \int \frac{d \bar{z}}{\bar{z}^{2}+b \bar{z}+c}
$$

(M.133) we have

$$
\begin{aligned}
& ||_{|r-x|}(T, S, R)=\frac{1}{2 R} \int_{C}^{1} d x \\
& x\left\{2 x-\frac{b}{2} \ln \frac{x^{2}+b z+c}{x^{2}-b x+c}+\frac{b^{2}-2 t-2 x^{2}}{2} \int_{-d}^{\int} \frac{d E}{z^{2}+b \bar{E}+c}\right\} \text {. }
\end{aligned}
$$

(M.114)

Therefore

$$
\begin{aligned}
& \operatorname{Re} \int_{\left.\left[\tilde{a}^{3}-2\right]\right\}}(T, S, R)=\frac{1}{2 R}\left\{1-\frac{b}{2} \int_{a}^{1} d z \ln \left[\frac{x^{2}+b x+c}{x^{2}-b x+c}\right]\right\}
\end{aligned}
$$

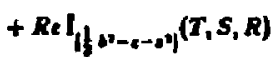

(M.115)

and

$$
\begin{aligned}
\left(b^{2}-c-s^{2}\right. & =b^{2}+\left(b-\frac{1}{R}\right) s-(1-d) \frac{T}{R} \\
& =\frac{1}{R}\left[z(T-1+b R)+\left(\left[b^{2} R-T\right)\right]\right.
\end{aligned}
$$

(M.116)

As well as

$z^{2}+4 z+c=\frac{1}{R}\{z(1-T)+T\} \quad z^{2}-b z+c=\frac{1}{R}\{z(1-T-2 l R)+T\}$

50

$$
\frac{z^{2}+b z+c}{z^{2}-b z+c}=\frac{s(1-T)+T}{s(1-T-2 b R)+T}
$$

Let

$$
N^{\prime}(x) \equiv 2\left[x(T-1+b R)+\left(\frac{1}{2} b^{2} R-T\right)\right]
$$

305

300 
then frow (M.115), (M.116), and (M.117)

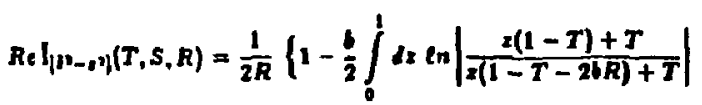

$$
\begin{aligned}
& \left.+R_{\in} h_{\left.N^{\prime}(\tau)\right)}(T, S, R)\right\} .
\end{aligned}
$$

Now both the numerator and denominator of $\frac{a(1-T)+T}{a(1-T-T)+7}$ are always ponitive $(0<0)$ so we can ansily integrate out this term. Sines

$$
\int_{a}^{1} d x \ln (a x+\rho)=\ln (\alpha+\rho)-1+\frac{\beta}{a} \ln \left(\frac{a}{b}+1\right)
$$

in Eeneral we have

$$
\int_{0}^{1} d z \ln \left(\frac{\alpha z+\beta}{\alpha^{\prime} s+\beta^{\prime}}\right)=\ln \left(\frac{a+\beta}{\alpha^{\alpha}+\beta^{\prime}}\right)+\frac{\beta}{\alpha} \ln \left(\frac{\alpha}{\partial}+1\right)-\frac{\rho^{\prime}}{\alpha^{\prime}} \ln \left(\frac{\alpha^{\prime}}{\beta^{\prime}}+1\right)
$$

with

$$
a=1-T \quad a^{\prime} \pm 1-T-2 b R \quad \beta=\rho^{\prime}=T
$$

(M.123)

for the integral under consideration. Since

$$
\alpha+\varphi=1 \text { and } a^{\prime}+\phi^{\prime}=1-2 b R
$$

we may write

$$
\begin{aligned}
\int_{0}^{1} d x \ln & \left(\frac{2(1-T)+T}{1(1-T-2 b R)+T}\right) \\
& =\frac{T}{1-T} \ln \left(\frac{1}{T}\right)-\ln (1-2 b R)-\frac{T}{1-T-2 b R} \ln \left(\frac{1-2 b R}{T}\right) \\
& =\frac{2 b R T \ln T}{(1-T)(1-T-2 b R)}-\frac{1-2 b R}{1-T-2 b R} \ln (1-2 b R)
\end{aligned}
$$

or lettins

$$
B \equiv S R=\frac{1-s}{2}
$$

$\int_{0}^{1} d x \ln \left(\frac{x(1-T)+T}{x(1-T-2 B)+T}\right)$

$$
=\frac{1}{1-T-2 B}\left[\frac{2 B T \ln T}{1-T}-(1-2 B) \ln (1-2 B)\right]
$$

\section{and $(M .120)$ becomes}

$R e l_{[P, x\}}(T, S, R)$

$$
\begin{aligned}
= & \frac{1}{2 R}\left\{1-\frac{1}{2 \bar{R}} \cdot \frac{B}{1-\bar{T}-2 B}\left[\frac{2 B T \ln T}{1-\bar{T}}-(1-2 \theta) \ln (1-2 \theta)\right] \quad\right. \text { (M.128) } \\
& \left.+\operatorname{Re} I_{\left[N^{\prime}(2)\right\}}(T, S, R)\right\}
\end{aligned}
$$

or

$$
\begin{aligned}
& \operatorname{Re}_{\left|z^{2}-s^{\prime}\right|}(T, S, R) \\
& =\frac{1}{2 R}\left\{1-\frac{1}{2 R} \cdot \frac{B}{s-T}\left[\frac{1-s}{1-T} T \ln T-s \ln S\right]\right. \\
& \left.+\operatorname{Re}_{\left[N^{\prime}(\alpha)\right\}}(T, S, R)\right\}
\end{aligned}
$$


since

$$
\begin{gathered}
B=\frac{1-S}{2} \quad b=\frac{B}{R} \quad 1-2 B=S \\
N^{\prime}(x\}=2\left\{x(T-1+B\}+\left(\left\{b^{2} R-T\right)\right\} .\right.
\end{gathered}
$$

(M.131)

When $S=1(B=b=0)$ this becomes

$$
R_{e} J_{\left.\left.\mid t^{2}-A\right\}\right]}(T, S=1, R)=\frac{1}{2 R}\left\{1-2 R_{e} J_{[x(1-T)+T]}(T, S=1, R)\right\} .
$$

M.6 G AND 6

G comet from, for instance, the $\gamma^{*}$ term of $M_{2}$ in Appendix $L$.

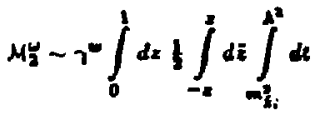

$$
\begin{aligned}
& x \frac{1-z}{\left(t-i x_{x}\right)(2-x)+\left(m_{p}^{2}-i\left(c_{1}\right) z+\frac{1}{4} g^{2}\left(\bar{z}^{2}-z^{2}\right)\right.} .
\end{aligned}
$$

Comparing the definition of $G$ from Table $L .1$ or the next section we see that

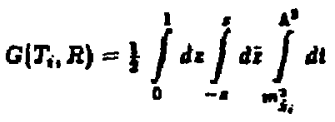

$$
\begin{aligned}
& \times \frac{1-z}{\left(1-i x_{i}\right)(1-z)+\left(m_{t}^{2}-i i_{i}\right) z+d^{3}\left(s^{3}-z^{2}\right)}
\end{aligned}
$$

(M.13t)

$$
\times \frac{1}{t+\frac{1}{1-x}\left[m^{2} f^{2}+\frac{l}{q^{2}}\left(z^{2}-x^{2}\right)\right]-i t}
$$

300 where $>$ D. Now use the Principal Value Theorem (M.50)

$$
\frac{1}{x-a \pm i d} \rightarrow \frac{1}{x-a} \mp i \times d(x-a)
$$

under the integral sign to give

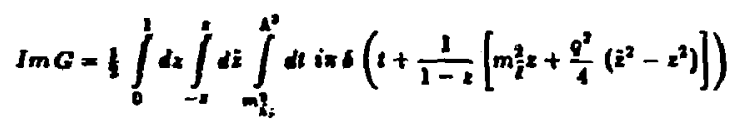

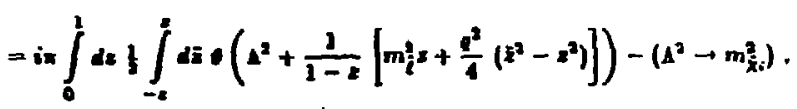

Since $\Lambda^{2} \rightarrow \infty \quad \varphi\left(\Lambda^{2}+\ldots\right)=1$ always and

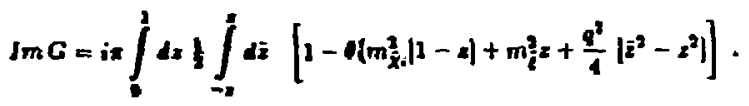

Now

$$
1-(x-a)=(a-2)
$$

hence

$$
\left.\operatorname{lm} G=i \pi \int_{0}^{1} d z \frac{1}{2} \int_{-1}^{R} d z \cdot\left[-\left(R \mid \bar{z}^{2}-z^{2}\right)+(1-z)+T_{z}\right)\right]
$$

Where we have divided through by $m_{x_{i}}^{2}>0($ ie. $\varphi(f(x))=\varphi(0 /(x))$ if $a>0)$. 
From (M.DS)

$$
W(x)=z^{2}-\frac{(1-2)+T_{2}}{R}>0 .
$$

Dividing through by $R\left(R>0\right.$ if $\left.q^{2}>0\right),($ M.139) becomes

$$
\operatorname{Im} G=i \pi \int_{0}^{l} d z \mid \int_{-\infty}^{e} d z\left(W(x)-z^{2}\right)
$$

This could be solved analytically (by ceser). From (M.135) and (M.s0)

$$
\begin{gathered}
\operatorname{Re} G=-1 \int_{0}^{1} d z \int_{-i}^{g} d E \ln \left|R\left(i^{2}-W(x)\right)\right| \\
T=\frac{m_{l}^{2}}{m_{x i}^{?}} \quad R=\frac{d^{2}}{4 m_{x_{1}}^{2}}
\end{gathered}
$$

$G$ is evaluated in s similar manner.

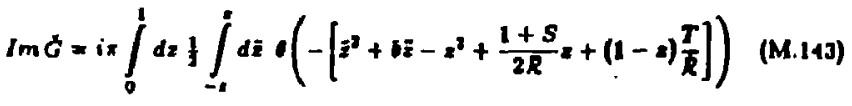

$$
\begin{aligned}
& \operatorname{Re} G=-\xi \int_{0}^{l} d z \int_{-1}^{l} d z \ln \left|R\left(\bar{z}^{2}-z^{3}\right)+\right|((1+S) z+(1-S) z)+T(1-z) \mid \\
& S=S_{i j}=m_{i_{i}}^{l} / m_{i j}^{2}, \quad T, R \text { as in(M.142) } \quad=\frac{1-S}{2 \hat{R}}
\end{aligned}
$$

Note that in ReG and Re $G$ that as $m_{i}^{2} \rightarrow \infty($ l.e. $T \rightarrow \infty)$ that

$$
\operatorname{Re} G, \operatorname{Re} G \rightarrow \sim \ln m_{i}^{*}
$$

which would seem unnatural for a term in a cross section.
M.7 G and $\mathbf{G}$ "Re-Defined", Lmiting forms.

All portions of the matrix elements are Enite es $m_{\dot{x}_{i}^{+}}, m_{\bar{x}_{i}^{0}} \rightarrow 0$ or $\phi^{2} \rightarrow \infty$ i.e. as $R \rightarrow \infty$ or $S_{i j} \rightarrow \infty$. Thete is troublesome point in the chargino sector a $m_{F} \rightarrow \infty$, i.e. as $T \rightarrow \infty$. We desire the infinite $-\frac{1}{1} \ln T$ contributions from $G$ and $G$ to cancel, at leat in the SUSY limit.

II we define

$$
G(T, R)=-1 \int_{0}^{1} d x \int_{-1}^{L} d z \ln \left[x(T-1)+1+R\left(i^{2}-x^{2}\right)\right]
$$

$\left.G(T, S, R)=-1 \int_{0}^{1} d z \int_{-\infty} d z \ln |T(z-z)+|((1+S) z+(1-S) \bar{z})+R\left(z^{2}-z^{2}\right)\right\}$

then

$$
\begin{aligned}
& G(T \rightarrow \infty, R) \rightarrow-f \int_{0}^{l} d x \int_{-1}^{\pi} d z \ln |x T|=-f \ln T+\frac{1}{4} \\
& G(T \rightarrow \infty, S, R) \rightarrow-\left|\int_{0}^{1} d x \int_{-\infty}^{k} d z \ln \right| T(1-x)|=-| \ln T+\frac{3}{4}
\end{aligned}
$$

Since all "real" processes will involve $\Delta G, \Delta G$ or $\Delta \bar{G}$ where

$$
\left.\Delta F \equiv F\right|_{i_{1}, b_{1}}-\left.F\right|_{i_{1, C_{2}}} \quad \tilde{F}(T, R) \equiv F(T, R)-F(T, R=0)
$$

for any function $F$, the constants will always cansel. It to only in numerical calculationa where, perhaps, the $\vec{l}_{1}$ (or $\vec{Z}_{2}$ ) ector is being artibclally examined by Itself, that a re-definition of these integral functions becomes desirable. The 


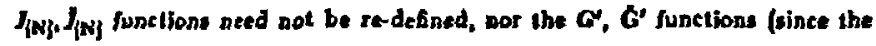
derivative of o constant vanistes). Thus we choose the redebinition

$$
G(T, R)=-\frac{1}{2} \int_{0}^{1} d x \int_{-1}^{1} d i \ln \mid x(T-1)+1+R\left(z^{2}-x^{2} \mid\right\}-\frac{1}{1}
$$

$$
G(T, S, R)=-1 \int_{0}^{1} d x \int_{-\infty}^{E} d E
$$

$\times \ln \left[T(1-a)+\left[\{(1+s)=+(1-s) \hat{x}\}+R\left(i^{2}-z^{2}\right)\right]-\frac{3}{4}\right.$.

For numerical investigations, if we use the defintions in (M.146)

$$
\begin{gathered}
G(T, R \rightarrow \infty) \rightarrow-1 \ln R+C_{o R} \\
G(T, R \rightarrow \infty) \rightarrow-1 \ln R+C_{C R} \\
G(T, S, R \rightarrow \infty) \rightarrow-1 \ln R+C_{o R}
\end{gathered}
$$

(M.153)

where

$$
C_{G R}=0.80684-1.57070370257 i
$$

and

$$
G(T, s \rightarrow \infty, R) \rightarrow-\ln s+C_{G s}
$$

where

$$
C_{G S}=0.75
$$

Note that when we use the defnition (Ag.152) that

$$
G(T, S, R \rightarrow \infty) \rightarrow-1 \ln R+0.05684-1.57079 i
$$

$$
\theta(T, s \rightarrow \infty, R) \rightarrow-\ln s
$$

In all of the $R \rightarrow \infty, S \rightarrow \infty$ limits $\Delta G \rightarrow 0$ and $\Delta \tilde{G} \rightarrow 0$ of tourse. Nole that $a m_{\bar{x}_{i}} \rightarrow \infty$ that $T_{i}, R_{i}, S_{i j} \rightarrow 0$. In this limit the quondam definitions of (M.146) and (M.147) would lead to

$$
\begin{aligned}
& G(R \rightarrow 0, T \rightarrow 0) \rightarrow-1 \int_{0}^{1} d x \int_{-2}^{E} \ln (1-x)=\frac{3}{1} \\
& G(R \rightarrow 0, T \rightarrow 0) \rightarrow-1 \int_{0}^{1} d z \int_{-x}^{\pi} d i \ln x=\frac{1}{4}
\end{aligned}
$$

$G(R \rightarrow 0, s>1, T \rightarrow 0) \rightarrow \frac{1}{4} \frac{2 s \ln s+3(1-s)}{1-s}$

$$
\text { C need not be contidered for } S<1 \text { since this is not used. }
$$

Note that

$$
\lim _{S \rightarrow 1} G(R \rightarrow 0, S, T \rightarrow 0)=\bar{C}(T \rightarrow 0, R \rightarrow)=\frac{1}{1}
$$

as desired.

If the $m_{\bar{x}_{i}^{+}}, m_{x_{i}^{0}} \rightarrow$ wo limils were thought to be of greater import then the $m_{i} \rightarrow \infty$ then a ditterent se-defintion could heve been made in which the constants $-1 / 4,-3 / 4$ is (M.151) and (M.152) could be interchanged to bring the limiting forms to zero. Using our revised definitions in (M.152)

$$
\begin{gathered}
G(T \rightarrow 0, R \rightarrow 0)=1 \\
G(T \rightarrow 0, R \rightarrow 0)=-1 \\
G(T \rightarrow 0, S>0, R \rightarrow 0)=\} \frac{s \ln s}{1-S} .
\end{gathered}
$$

Which are much more aymmetric than was the case with the functions as they 
were previoush defined.

In conclusion we present certain $y^{2}=0$ limiting cases of interat:

$$
\begin{aligned}
& G(T, 0)=-1\left[\ln T-\frac{\ln T^{2}+(3-T)(1-T)}{2(1-T)^{2}}\right]-\frac{1}{1} \quad G(T \rightarrow 1,0) \rightarrow-\frac{1}{4} \\
& \bar{C}(T, 0)=-\frac{T^{2} \ln T^{1}+(3 T-1)(T-1)}{1(T-1)^{2}}-\frac{3}{4} \quad \sigma(T \rightarrow 1,0) \rightarrow-\frac{7}{4} \\
& I_{|1|}(T, 0)=\frac{T \ln T+1-T}{(1-T)^{2}} \quad J_{|1|}(T \rightarrow 1,0) \rightarrow 1 .
\end{aligned}
$$

The formulae developed in this chspter heve been used to plot the more impor. tant of the integral functions over a sange of parameles regloes. It thould be mentioned that the Fejnman diagrams onay be equivalently expressed in terme of what way be becoming atandardized uet of integral functions originally put lotth by Passarino and Veliman."

\section{FIGURE CAPTIONS}

1. Tree Level Vertex with more than one Neutralino.

2. Typical diagram contributing to $f_{|x|}$ term.

3. $G(T, R)$ vs. $m_{i}$ for $m_{\bar{x}}=0.1,20 \mathrm{GeV}$.

$$
\begin{aligned}
& \mathrm{T}=\frac{\mathrm{m} ?}{\mathrm{~m} !} \\
& A=\frac{c^{2}}{4 m_{x}^{2}} \\
& f^{2}=m y=(00 G \mathrm{GV})^{2}
\end{aligned}
$$

4. $G^{\prime}(T, R)$ ve. $m_{i}$ for $m_{\tilde{X}}=0.1,20,45,50,100 \mathrm{GeV}$.

$$
T=\frac{m_{i}^{2}}{m_{\bar{x}}^{2}} \quad R=\frac{e^{2}}{4 m_{x}^{2}} \quad v^{2}=m_{z}^{2}=(00 \mathrm{GeV})^{2}
$$

B. $C(T, S, R)$ vs. $m_{i}$ for $m_{\tilde{x}_{i}}=0.1 \mathrm{GeV}$ and $s=1,10000$. $T=\frac{m_{i}^{2}}{m_{x_{i}}^{2}} \quad R=\frac{c^{2}}{4 m_{x_{i}}^{2}} \quad s=\frac{m_{x_{i}}^{2}}{m_{X_{i}}^{2}} \quad I^{\prime}=m_{z}^{2}=(\infty \mathrm{GeV})^{2}$

6. $G^{\prime}(T, S, R)$ ve. $m_{E}$ for $m_{\bar{z}}=0.1 \mathrm{GeV}$ and $s=1,2$ (Indistinquishable).

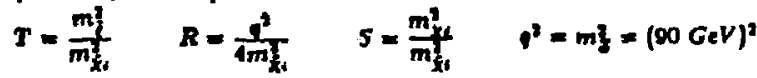

7. $\bar{G}(T, R)$ ve. $m_{i}$ for $m_{\bar{x}}=1.0 \mathrm{MeV}$.

$$
T=\frac{m^{2}}{m_{x}^{2}} \quad R=\frac{s^{3}}{4 m_{x}^{2}} \quad P^{2}=(92.0 \mathrm{GeV})^{\prime}
$$

8. $R \times I_{\{\tilde{z}=2 \text {-2\} }}(T, R)$ vis. $m_{\bar{x}}$ for $m_{i}=20,40,60,80,100 \mathrm{GeV}$.

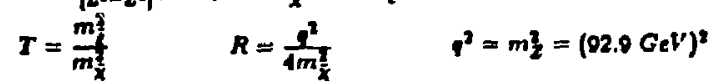

9. $f_{1,1}(T, S, R)$ ve. $m_{i}$ for $m_{\bar{x}}=0.1 \mathrm{GeV}$ and $s=1,6 \times 10^{6}, 8 \times 10^{6}, 20^{6}$.

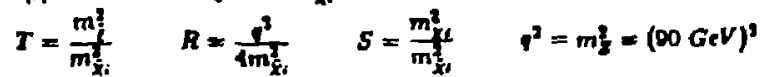

10. $I_{[1]}(T, S, R)$ ve. $m_{i}$ for $m_{\overline{x_{i}}}=0.1 \mathrm{GeV}$ and $s=1.4,1.6$.

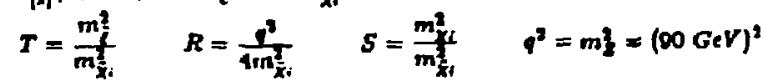




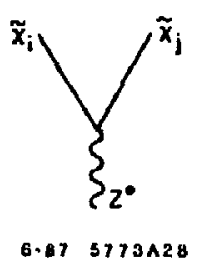

Fig. M.1

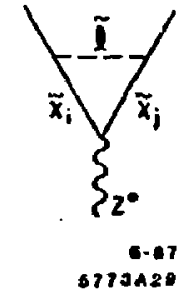

Fig. M.2 


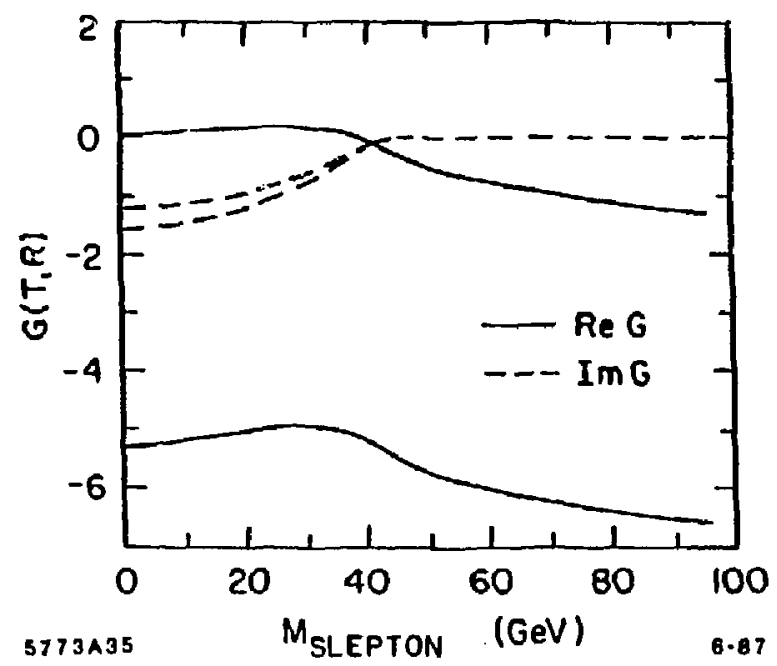

Fig. M.3

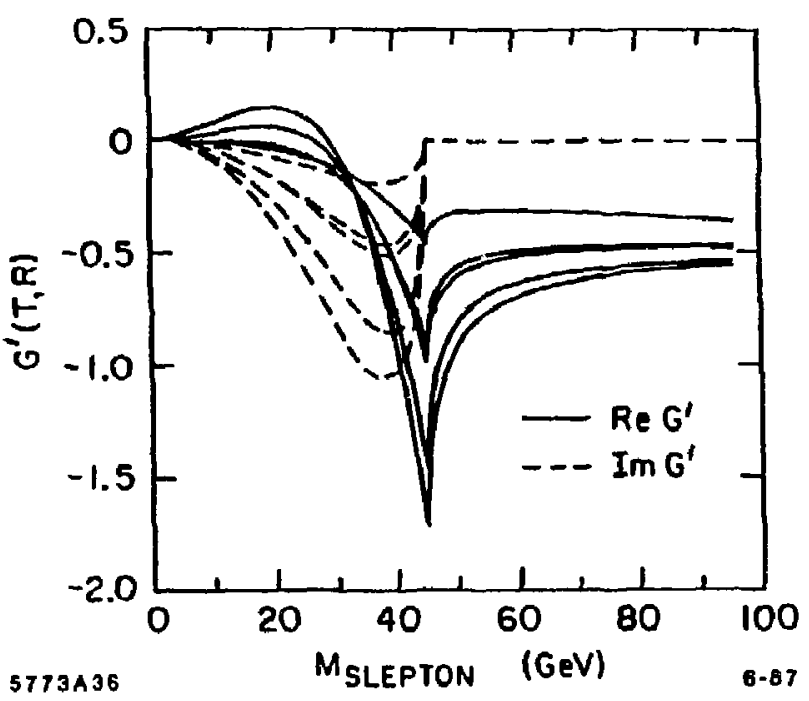

Fig. M.4 

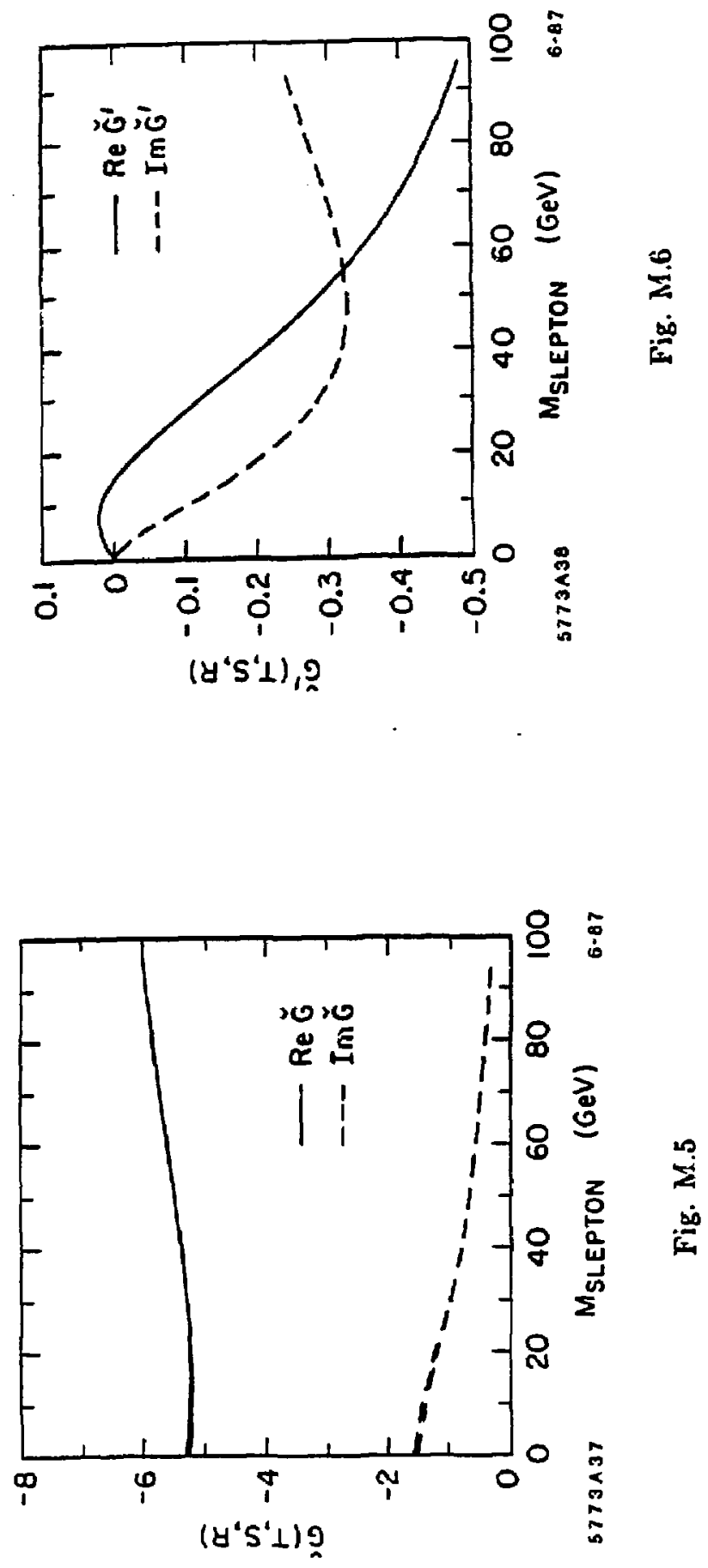


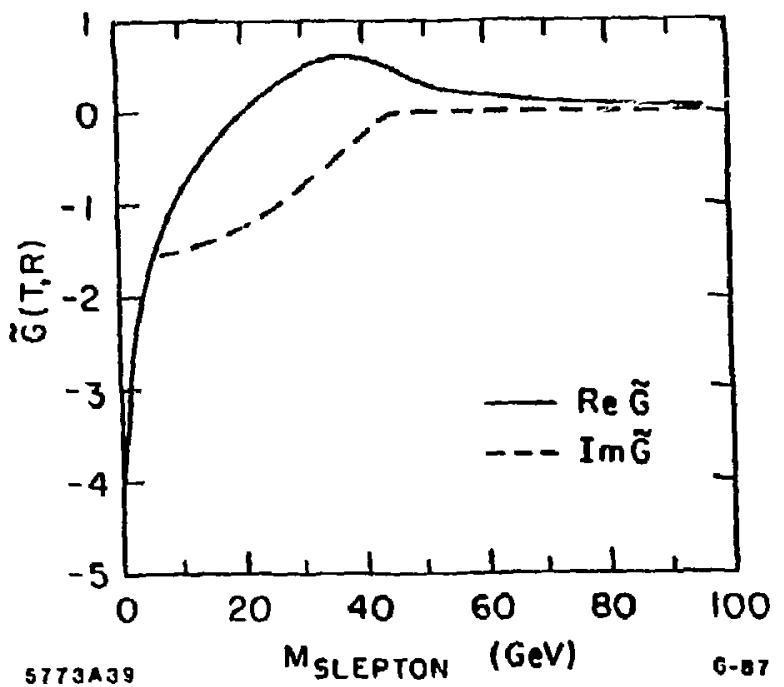

Fig. M.7

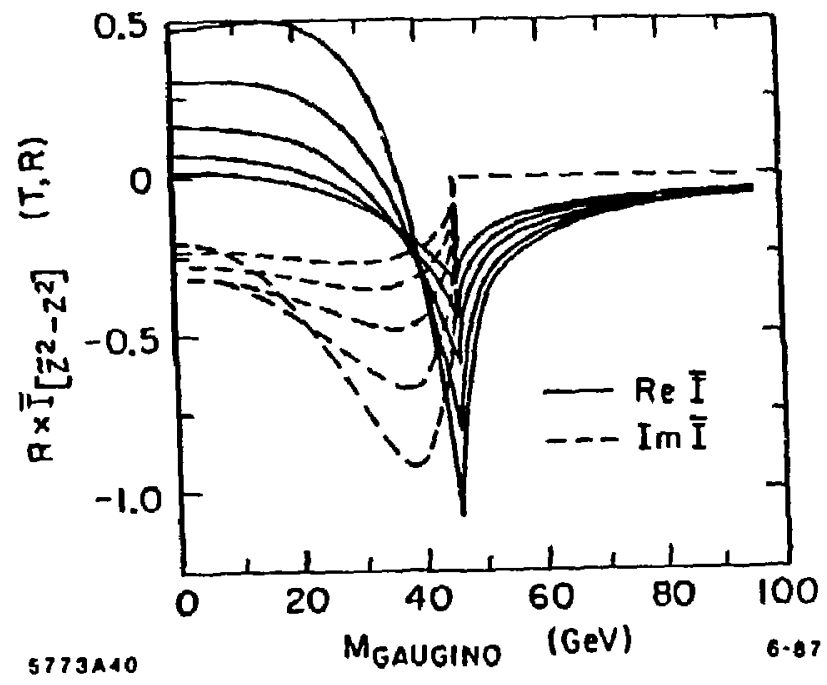

Fig. M.8 


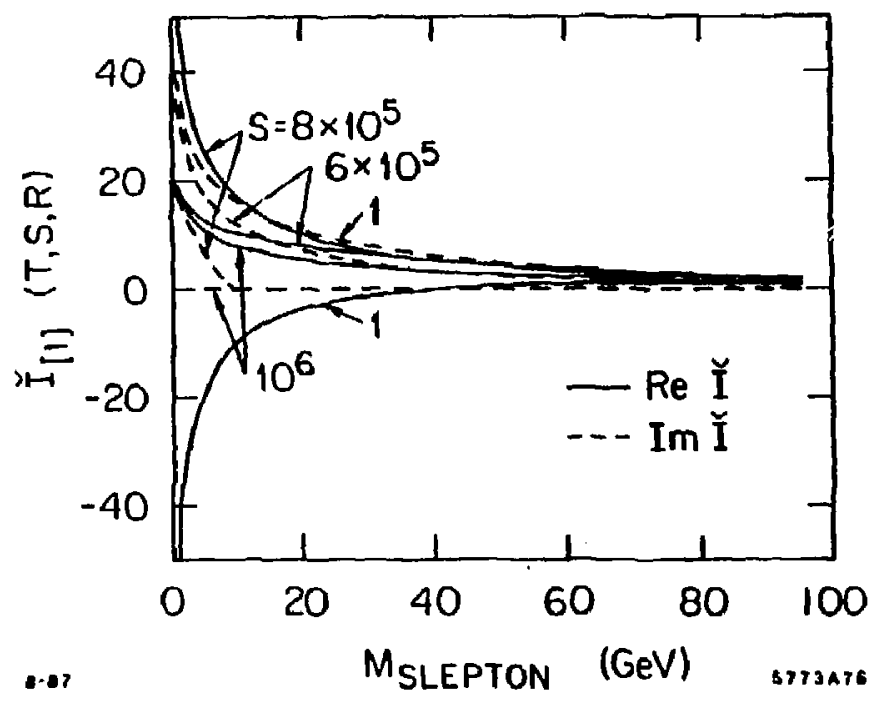

Fig. M.9

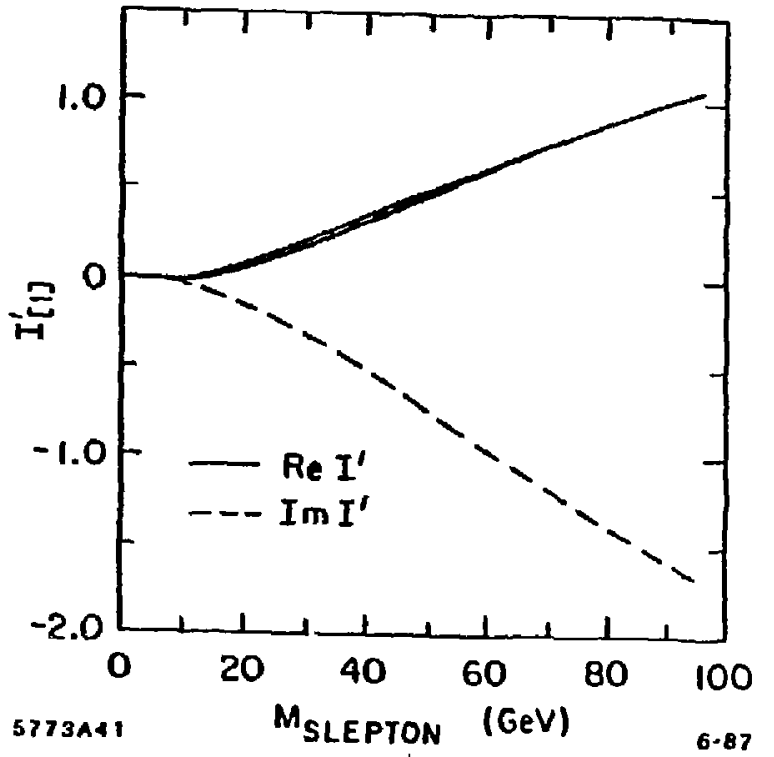

Fig. M.10 


\section{APPENDIX N}

Bachround

We wh to consider the difficultie involved in observing $e^{+} e^{-} \rightarrow \mu r$ at SLC (or LEP) while operating at a $Z^{0}$-resonance whereat $\left(p_{1}-+p_{a}\right)^{2}=m_{3}$ which, for the purposes of numerical estimates, we will take $a(90 \mathrm{G}: \mathrm{V})^{2}$ in this appendix. The detector signal is a $\mu^{ \pm}$and a f $^{7}$ exiting back-to-back, each with essentially half of the total energy and with no gnissing mass (from $\nu^{\prime} \mathrm{b}, 7^{\prime} \mathrm{b}, \mathrm{j}^{\prime} \mathrm{b}, \mathrm{etc}$.)

Thb aignal may be mimicked by the far mure common decay mode $z^{0} \rightarrow \mathrm{rr}$ with the subsequent decay of one or both of the r's into a muon and neutrinos. Since the $T$ will travel $\sim 0.25 \mathrm{~cm}$ prior to decay, and auch a kink would be unobservable if the decay products of the, do not come out at a larze angle with respect to the r'a direction, the event will resemble a muon and the decay products ol ar. In either process the r's mut emerge back-to-back, bolh with $E=45 \mathrm{GeV}$ (to within a lew MeV), but wince alf decay modes of the tau Involve at least one undelectable particle (the tau neutrino), and many $\left(r \rightarrow \mu \nu \nu_{\text {, }} e \nu \nu\right.$, ete. $)$ tive rise to several euch states, accurate enercy and momentum reconstruction is dificult. There is no problem with the modes $r \rightarrow$ HADRONS of $r^{+} \rightarrow e^{+} v_{c} v_{r}$, but $r^{+} \rightarrow \mu^{+} \nu_{\mu} D_{r}$ providea substantial background. Some of the $\mu^{+}$will have virtually all of the $r^{+}$momentum (sofl neutrinas) and so the event will oppeas we $e^{+} e^{-} \rightarrow r^{+} \mu^{-}$instead of $r^{+} r^{-}$.

One method of reducing the becheround would be to etiminate all cignals which have an opening angle which is discernibly different than 180 decrees. The dificulty in distinguishing the two decay modes $Z^{0} \rightarrow r \mu$ and $Z^{0} \rightarrow r r \rightarrow r \mu+$ coft neutrinas is that both involve at least one tav reconstruction. Since euch a reconstruction will necessarily introduce an error in the inferred tau momentum angle (which varies over the sanze of the detector) there will be some $\Delta f$ for

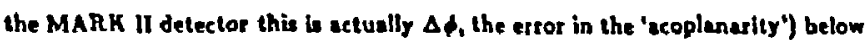
which it will be impostible to distinguish a back-to-back event trom one whose opening angle is $\pi-\Delta C$. For $\&$ buckground event the opening angle will range up to approximately twice what it was for a genuine sf event. Thus we will bs able to experimentally eliminate those events with the largest opening anzles. But these are precisely the events which will be most easily difierentiated on the basis of calorimetric information (since events with larger opening angles will tend to be those with atifrer neutrimos). Thus it appeare that angular information will not be useful in dizcriminating the background from the actual aignal." An analogous argument applies to using the impact parameter.

It appears that we must rely opon enerzy and momentum considerations to exclude the background contribution. The dificulty of eliminating the apurious component depends crucially upon the enercy resolution of the detector and what the tail end of the energy distribution of $r(15 \mathrm{GcV}) \rightarrow \mu \nu \nu\left(p_{\mu}=45 \mathrm{GeV}\right)$ looks

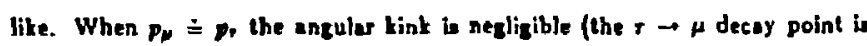
$2.5 \mathrm{~mm}$ (rom the interaction region) and will be indiscernible. The time of tight (delay because $\left.m_{1}>m_{\mu}\right)$ difference in $\sim 0.25 \mathrm{~cm} /\left(1-\beta_{r}\right)$ with $\beta_{r}=\sqrt{1-\left(1 / \gamma_{t}\right)}$ where

$$
7_{r}=4.5 \mathrm{GeV} /(\mathrm{m}, \approx 1.784 \mathrm{GeV}) \approx 25.2
$$

Therelore

$$
\theta, \approx 1-\frac{1}{2 \gamma^{2}}
$$


and

$$
1-\beta_{1}=\frac{1}{2 \gamma^{2}}
$$

yielding

$$
\Delta t=\frac{2.5 \times 10^{-3} \mathrm{~m}}{3 \times 10^{+6 / \mathrm{m} / \mathrm{a}}} \cdot \frac{1}{2(25.2)^{2}} \times 6.6 \times 10^{-16} \mathrm{etc} .
$$

So the delay time is about 6.6 femtoseconds and experimentally unobservable.

We need to calculate the energy spectrum of the $\mu^{ \pm}$produced from the decay of a 7 . We will perform an ideslized background calculation. We will consider only events which heve passed cuts imposed by the detector topolosy and ignore

1. Beam width (i.e. assume that the beam energy is precisely known).

2. Bremsstrahlung. The $\mu$ and $r$ will bremsulrahlung is the otrong magnetic Geld of the delector (even over the $\mathbf{2 . 5}$ mm decay distance).

3. Chance of particle interaction (imperfect vacuum) in detector (cecondary cattering).

4. Imperfect particle identification.

At SLC the $c^{-}$beam will be $100 \%$ polatized (say in the $\bar{Z}$ direction). Thus to make a (spin $=1) z^{0}$ only the $e^{+}$component with spin in the $+\hat{z}$ direction also (almost all of it) will be used. As a result nearly all of the $Z^{0}$ will be created polnrized in the $+\bar{Z}$ direction and at reti. Examining the helicity states of $\mu^{-}, r^{+}$

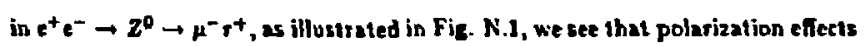
are expected to be important.

Let us consider the I decaying at sest and then "boost" this to the lab $\left(p_{1}=45 \mathrm{GeV} / \mathrm{c}\right)$ frame as in Fig. N.2. We know that the $\mathrm{z}$ will likely be polarized. The normalized number distribution of the muons in the tau rest frame ${ }^{47}$ (using the 1-Fermi Lagrangian, which b an adequate approximation here, and $\left.m_{p}<m_{p}\right)$ is

$$
N_{A}=x^{2}\left[3-2 x \pm a(1-2 x) \cos \theta_{B \operatorname{son} 1}\right] d x d \operatorname{tos} \theta
$$

$$
\begin{gathered}
+\operatorname{los} r^{-} \rightarrow \mu^{-} \\
-\operatorname{tar} r^{+} \rightarrow \mu^{+} \\
a=\text { Oeon here } \\
a=0 \text { unpolarized of polurization }
\end{gathered}
$$$$
a=1100 \% \text { polarized) }
$$

$$
\mp=\frac{E_{i}}{\sqrt{m_{i}}} .
$$

$E_{i}=E_{\mu}$ in r test frame, prios to boosting by $/ \mathrm{mz}$.

$$
f m_{*}=\max \left|E_{i}\right| \text { in real frame co } 0 \leq x \leq 1 \text {. }
$$

Therefore

$$
\begin{aligned}
\frac{d N_{\mu}}{d E_{i}}= & \frac{d N_{\mu}}{d x}\left(\frac{x}{E_{i}}\right)=\frac{2}{m_{r}}\left[\frac{2 E_{i}}{m_{i}}\right]^{2} \\
& \times\left[3-2\left(\frac{2 E_{i}}{m_{i}}\right) \pm a\left(\begin{array}{c}
\cos \theta \\
\cos (\theta+x)
\end{array}\right)\left(1-2\left[\frac{2 E_{i}}{m_{i}}\right]\right)\right] d \cos \theta \\
= & \frac{16 E_{i}^{2}}{m_{r}^{2}}\left[3 m_{1}-4 E_{i}-a\left(m_{1}-4 E_{i}\right) \cos \theta \mid d \cos \theta .\right.
\end{aligned}
$$

Nole that the "- sign holds for both $\mu^{+}$and $\mu^{-}$because for $\mu^{+}$the polarization is in the momentum direction (ane $a$ boost) whereas for $\mu^{-}$it is in the opposite direction. This just compensates for the sign difference in (N.S).

390 
The (normalized) number of $\mu$ 's in the range $(0.0+d 0$ ) (i.e. r rest frame) with enercy $E_{i}$ is

$$
d N_{p}\left(E_{i}, 0\right)=\frac{16 E_{i}^{2}}{m_{i}^{4}}\left[3 m_{1}-1 E_{i}-a\left(m_{i}-4 E_{i}\right) \cos 0\right) \frac{2 \pi d \cos U}{d n} d E_{i}
$$

(nince there is no $\phi$ dependence and $\left.\int d n=4 \pi\right)$.

We now boost (in $\bar{z}$ direction sny) up to $E_{\mathrm{p}}=m_{2} / 2$. In zeneral ${ }^{40}$

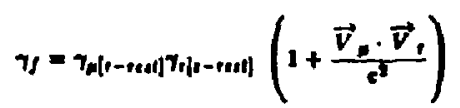

and since $\rho_{\mu} \approx \rho_{1} \sim 1$

$$
\gamma=\frac{E_{i}}{m_{p}} \cdot \frac{\mid m_{r}}{m_{i}}\left(1+\rho_{m} A_{1} \cos 0\right) \sim \frac{E_{i} E_{z}}{m_{\mu} m_{r}} \frac{(1+\cos \theta)}{7} .
$$

Let $E_{f}$ be the $6 n a l$ enercy of the $\mu$ in the lab $\left(2^{\circ}\right.$ at reat) frame

$$
E_{f}=m_{\mu} \eta_{f}=\left\lfloor E_{i} \frac{m_{z}}{m_{p}}(1+\cos \theta)\right.
$$

(rememberisg that $O$ is in the $r$ test Irame). The probability of the final state having energy $E_{f}$ in the lab frame, given energy $E_{i}$ and angle in the r rest Irame, is

$$
\left.E_{f}=\int \text { (Prob. of } E_{1}\right)(\text { Prob. of } 0)
$$

up to a nosmaliation, where $E_{f}=f E_{i} \frac{m_{n}}{m_{i}}(1+\cos \theta)$ from (N.12). Letting

$$
y=\frac{2 E_{l} m_{1}}{m_{I}(1+\cos \theta)}
$$

this condition is $E_{i}=y$.
The probability of $E_{f}$ being the Enal $\mu$ enercy is, therefore, ( $N$ is a normal. [eation (actor)

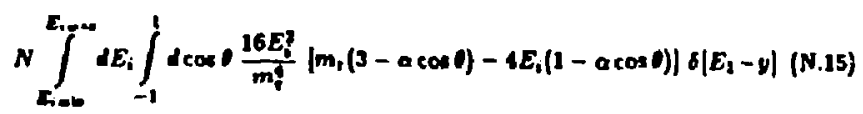

with $E_{i=i n}=0$ and $E_{i=u}=m_{r} / 2$. Letting $z=\cos \theta$ The energy constraint equation becomes

$$
v=\frac{3 E_{j} m_{r}}{m_{2}(I+x)}
$$

and the number of wuone expected at a given lab enercy it

$$
\begin{aligned}
& \frac{d N_{H}}{d E_{j}}=N \int_{0}^{m_{i} / 2} d E_{i} \int_{-i}^{l} d z \frac{16 E_{i}^{2}}{m_{i}}\left[m_{1}(3-\alpha z)-4 E_{i}(1-\alpha z) d\left|E_{i}-y\right|\right. \\
& =N \int_{-1}^{m, / 2-g} d\left(E_{i}-v\right) \int_{-1}^{l} d x \frac{16 E_{i}^{z}}{m_{i}}\left[m_{r}(3-a x)-4 E_{i}(1-a x] \delta\left|E_{i}-\eta\right|\right. \\
& =N \int_{-1}^{t} f d z \frac{16}{m_{i}}\left[\frac{2 E_{f} m_{1}}{m_{z}(1+2)}\right]^{2} \\
& \times\left[m_{r}\left(3-a_{2}\right)-4 \cdot \frac{2 E_{f m_{r}}}{m_{2}(1+2)}\left(1-a_{2}\right)\right] \cdot\left(\frac{m_{r}}{2}-\frac{2 E_{j} m_{r}}{m_{2}(1+2)}\right) \text {. }
\end{aligned}
$$

The argument of the theta-function must exceed aero requiring that

$$
\frac{m_{r}}{2}-\frac{2 E_{J} m_{r}}{m_{z}(1+2)}>0 \Rightarrow x>\frac{4 E_{J}}{m_{z}}-1
$$

Therefore

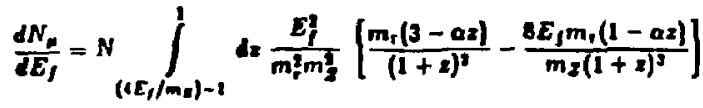


and letting $w=1+z$

$$
\begin{aligned}
& \frac{d N_{\omega}}{d E_{j}}=N \frac{E_{f}^{2}}{m_{j} m^{g}} \int_{1 E_{f} / m_{s}}^{2} d v\left[\frac{s-\alpha(\omega-1)}{\omega^{2}}-\frac{E_{f}}{m_{I}} \frac{1-a(w-1)}{\omega^{2}}\right] \\
& =N \frac{E_{j}}{m_{\mathrm{r}} m_{z}^{2}} \int_{1 \varepsilon_{l} / m_{\mathrm{g}}}^{z} d w\left[-\frac{m_{2 a}}{\omega}+\frac{(3+a) m_{2}+\Delta a E_{l}}{\omega^{2}}-\frac{(1+a) E_{l}}{\omega^{2}}\right] \\
& =N \frac{E_{f}}{4 m_{1} m_{\xi}}\left[\operatorname{tam} E_{f} \ln \frac{2 E_{l}}{m_{3}}+6(a-1) m_{2} E_{J}\right. \\
& \left.+4(1-3 \alpha) E_{j}^{3}+2 m_{\alpha}^{2}\right]
\end{aligned}
$$

Let

(N.18)

$$
\eta=\frac{N}{4 m_{\mathrm{r}} m_{!}} .
$$

The normalization is established by requiring that

$$
\int_{0}^{m_{E} / 2}\left(\frac{d N_{L}}{d E_{f}}\right) d E_{f}=1
$$

and so

$$
\begin{aligned}
& \eta \int_{0}^{m_{f} / 2} d E_{f} E_{J} \\
& \times\left\{4 a m_{Z} E_{j} \ln \frac{2 E_{J}}{m_{Z}}+6(a-1) m_{z} E_{J}+4(1-3 a) E_{j}^{2}+2 m_{z}^{2}\right\}=1 .
\end{aligned}
$$

Performing the integration yield's the result

$$
1=q\left\{\frac{m i}{144}(\theta+a)\right\}
$$

s33 or

$$
=\frac{144}{m !(0+a)}
$$

which, when used in (H.3S), sivas

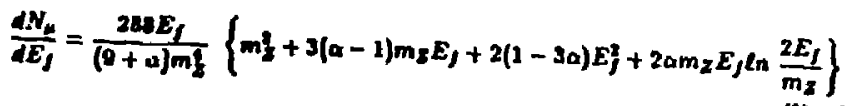

Where $a=0$ for the unpolarized case and $a=3$ in the completely polarized case.

For $a=0$ :

$$
\frac{d N_{l}}{d E_{l}}=\frac{32 E_{l}\left(E_{l}-m_{z}\right)\left(2 E_{I}-m_{z}\right)}{m_{l}} \text { (unpolarized cure). }
$$

For $\alpha=1:$

$$
\frac{d N_{\mu}}{d E_{f}}=\frac{144}{5} \frac{E_{f}}{m_{l}}\left\{\left(m^{2}-4 E_{j}\right)+2 m_{z} E_{j} \ln \frac{2 E_{f}}{m_{2}}\right\} \text { (polarized cast). }
$$

These distributions are depicted in Fig. N.3.

Wee are interested is those $\mu^{\prime}$ will $E_{\mu} \doteq E_{r}=m_{z} / 2$. The number, $N(\Delta c)$, of $\mu$ 's within energy $\Delta c$ of $\mathrm{m}_{\mathrm{z}} / 2$ is

$$
N(\Delta c)=\int_{(m x / n)-\Delta c}^{m_{2} / 2} d E_{j} \frac{d N_{p}}{d E_{j}}
$$

314 
Therefore

$$
\begin{aligned}
& N(\Delta c)=1-\frac{288}{0+a} \int_{c}^{\left(m_{0} / 2\right)-\Delta_{t}} \frac{d E_{f} E_{f}}{m !} \\
& \left.\times\left\{m_{2}^{1}+3(a-1) m_{2} E_{l}+2(1-3 a) E\right\}+2 a m_{2} E_{f} \ln \frac{2 E_{l}}{m_{3}}\right\} \\
& =1-\frac{288}{9+a} \frac{1}{m !} \\
& \times\left\{\left|m_{z}^{2} E_{\}}^{2}+(a-1) m_{z} E_{\}}^{3}+\right|(1-3 a) E_{\}}\right. \\
& \left.+\left(\frac{3}{3} a m_{2} \ln \frac{3}{m_{2}}\right) E_{f}^{3}+2 a m_{2} E_{j}^{3}\left(\frac{1}{3} \ln E_{f}-\frac{1}{6}\right)\right\}\left.\right|_{0} ^{\left(m_{2} / 2\right)-\Delta a}
\end{aligned}
$$

and expending all terms to order $(\Delta e)^{2}$ yields (eventuelly)

$$
\begin{aligned}
& N(\Delta c)=\frac{3-a}{9+a} \cdot 24 \frac{\Delta c^{2}}{m \xi} \\
& a=0 \text { tives } N(\Delta c)=\frac{(\Delta x)^{2}}{m^{2}} \\
& 0=1 \text { tives } N(\Delta r)=\frac{21}{3} \frac{(\Delta r)^{2}}{m_{2}^{1}} \text {. }
\end{aligned}
$$

Thus polarization effects decrease the background by $a$ factor of $5 / 3$.

The energy-momentum resolution for atif muons for the Mark II detector at the SLC will be, at best, (once the elose vertex detector has been installed) ${ }^{21}$

$$
\frac{\Delta p}{p} \geq 0.1 \times \text { per } G e V
$$

For muons in the $45 \mathrm{GeV}$ range this implies that

$$
\frac{\Delta P}{F} \geq \mathbf{5 \%}
$$

(There in an additional 1.4\% error anticipated from wulliple acatterinzs, within the drift chamber alone, which must be added in quadrature.) Taking the "cuper. optimal" morpentum resolution, $\triangle$ copT, at SLC to be $5 \%$ then

$$
\Delta c_{O H T}=5 \%\left(\frac{m_{2}}{2}\right)=0.05\left(\frac{m_{2}}{2}\right)
$$

and we observe frow (N.21) that

$$
\begin{aligned}
& N(\Delta \text { COPT })=\frac{3-a}{0+a} \cdot 21\left(\frac{0.0 s \cdot \mathrm{m}_{2}}{m_{2}}\right)^{\prime}=\left(\frac{1-a}{D+a}\right) 1.5 \times 10^{-2} . \\
& \text { For } \alpha=0 \quad N(\Delta \text { Cort })=5 \times 10^{-0} \\
& \text { For } Q=1 N\left(\Delta \varepsilon_{O F T}\right)=3 \times 10^{-3} \text {. }
\end{aligned}
$$

Note that the difierence between $E_{\mu}$ and $m_{z} / 2$ (due to $m_{1} \neq m_{\mu}$ ) bo only about $17 \mathrm{MeV}$, lar below the energy resolution.

Now

$$
\begin{array}{cl}
B R(Z \rightarrow r)=3 \% & B R(Z \rightarrow \mu \mu)=3 \% \\
B R(r \rightarrow \mu \sigma \nu)=17.5 \% & B R(\tau \rightarrow C O \nu) \cong 17.5 \%
\end{array}
$$

and therefore an expected ample (ultimate fiducial yearly yield at SLC) of 
$10^{5} Z^{0}$ o yitids $30,000 \mathrm{rr}$ events. We expeet roushly

$$
10^{6} \times 0.03 \times 2 \times 0.175 \times\left(3 \times 10^{-3}\right) \sim 30
$$

misidentified stiff $\mu^{\prime}$ from $e^{+} c^{-} \rightarrow$ ir events (many of which will go undetected due to their decay configuration). We also expect $-10,000 \mu \mu$ events. From chapter three recall that

$$
\frac{a_{u r}}{a_{u p}} \leq 3 \times 10^{-1}
$$

and so we would expett fewer than

$$
10^{4} \times 0.03 \times 2 \times 3 \times 10^{-4}=2 \times 10^{-1} \text { evente per year. }
$$

We may simultaneously searcb for both $\mathrm{r}^{+} \mu^{-}$and $\mathrm{r}^{-} \mu^{+}$events, accounting for the additional factor of two. Azain some of this signal will be bat.

The question arises as to whether we could improve these results if the principal mixing wose in the $\tilde{e}-\tilde{\mu}$ sector rather than the $\tilde{\mu}-\tilde{F}$ sector. Since faetors of the lepton gasses have cancelled from (N.24), the ande expression is

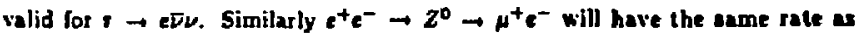
$e^{+} e^{-} \rightarrow 2^{0} \rightarrow{ }^{+} \mu^{-}$however the primary background will now arise from

$$
\begin{aligned}
c^{+} c^{-} \rightarrow Z^{0} & \rightarrow t^{+} t^{-} \\
t^{+} & \rightarrow \mu^{+} \mathrm{J \nu} \\
\tau^{-} & \rightarrow e^{-E \nu} .
\end{aligned}
$$

We expect that ( $P$ means "probability of $)$

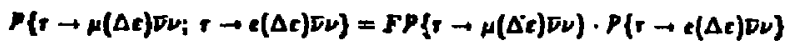

where $F$ is a factos of order unity. This deacribes events in which the taus emerge back-toback and decay into an electron and muon, both of which retain almost all of the moweatum of their pupent thus, and appear to continue diametrically back-to-back with no apparent energy bas or deviation. From (N.21) we know that

$$
N(\Delta r)_{(p r)}=\frac{3-\alpha}{0+\alpha} \cdot B \frac{(\Delta p)^{2}}{p^{3}}
$$

therefore

$$
N(\Delta r)_{(+p)}=\left(\frac{3-\alpha}{9+\alpha}\right)^{2} \cdot 36\left(\frac{\Delta p}{p}\right)^{2}
$$

For $a=1$ this becoon

$$
N(\Delta c)_{(\mu p)}=1.1\left(\frac{\Delta p}{p}\right)^{4}
$$

which, for a 5\% etror give

$$
N(\Delta p)=0 \times 10^{-6} .
$$

From the branching ration in (N.34) we expect about

$$
10^{6} \times 0.03 \times 2 \times 0.175 \times 0.175 \times\left(9 \times 10^{-6}\right) \sim 2 \times 10^{-2}
$$

misidentifed $e^{+} e^{-} \rightarrow e^{ \pm} \mu^{\mp}$ events per arople of $10^{*} 2^{\circ}$. Comparine with (N.37) we see that this could, in theory, be as much as an order of magnitude 
lest that tice signal rate. This conclusion is, of course, based upon somewhat idealized ("ouper-optimal") background ealimates and praduction rates. It should be noted that, in partial compensation, it will be substantially eatier to eliminate background on the besis of angular information in this syatem since no r-track momentum reconstruction will be required.

\section{FIGURE CAPTIONS}

1. The decay $2^{0} \rightarrow r \mu$ in the Jaboralory rest frame illustrating the configurations which are and are nol helicity-suppreased.

2. The decay $r \rightarrow \mu[\nu$ in $r$ reat frame.

a the angle of the $m$ momentum with respect to r polarization factor.

is also the boost direction angle for the $\mu^{+}$case.

$+\pi$ is boost diraction angle for the $r^{-} \rightarrow \mu^{-}$case.

3. The distribution of emergent muons, $\frac{d W_{2}}{\pi E_{l}}$, from $r \rightarrow \mu \nu \nu$ versus $E_{l}$, the muon enercy in the laboratory frame.

The detree of polarization of the $r^{ \pm}$it a. 


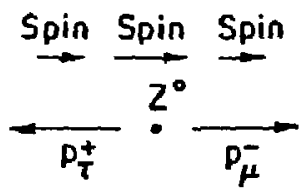

(a)

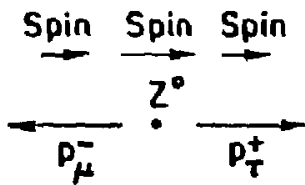

(b)

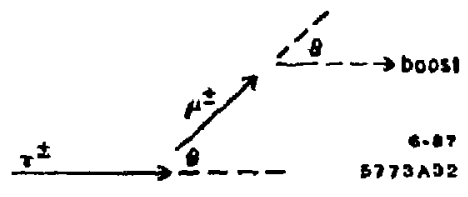

Fig. N.1

6773431

Fig. N.2 


\section{APPENDIX 0}

Alatrix Element for $\mu \rightarrow \mathrm{e}$.

\subsection{InTRODUCtiok}

$A$ one loop calculation of the mej vertex ha been petformed. Unlike the

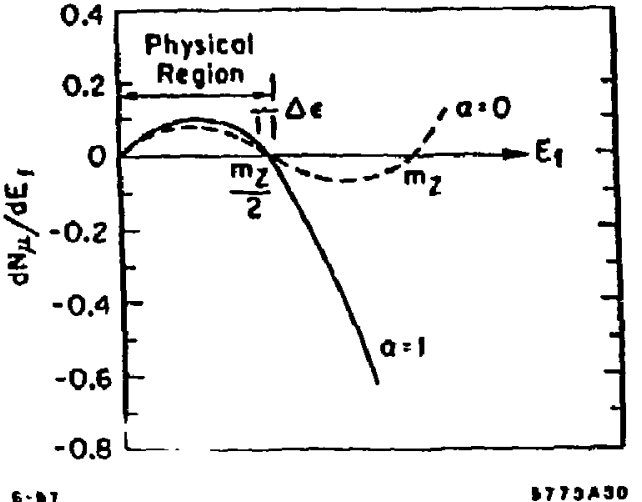

Fig. N.3 prelercoission in the $Z \rightarrow$ ur compulations, here we shall not neglect the massea of the exhernal termions and asume the photon to be on-shell. The result may be added to the efiective Lagrangian of the theary and is valid whenever the keptons are on-shell (when all the ctales are oft-shell the result $1 \mathrm{comewhat}$ more involved since some portion of the $m_{i}^{2}$ terms will be replaced by $p_{i}^{2}$ and cauce invariance will be mote diticult to emplay a a chect on the snal form of the resule). The calculation was performed with the ino-component formalism for a purely SQED theory. We know from considerations of Lorentz and gave Invariance thet the results may be placed in the form:

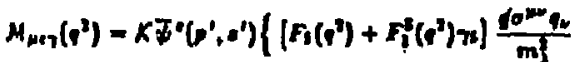

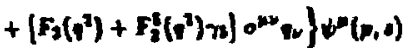

$$
K=-\frac{a^{3 / 2} \sin \theta \cos \theta}{144 \sqrt{x}} \quad q_{u}=p_{u}-\phi_{u}^{\prime}
$$

whe is the $\hat{E}-\hat{\mu}$ mixir: angle.

There are Iwenty Weyl-spinar diagrams in all, Ialling into the catagories of triangle and he-correction contributions. Each conkribules a piece to bath the $F_{1}$ and $f_{2}$ trime as well as other pleces which would be disallowed by our general considerations. These can be showa explieitly (and rather laboriously) to cancel 
among themelve. To facilitate this it was found useful develop a projection technique, similer to that employed by Brodaky and Sulliva," to project out of each two-component expression that portfon which contributed to each of the Lerms in (0.1). The tangle of algebra which followed was made tractable by judicious (i.c. extensive) ues of the eymbolic menipulation toutine REDUCE and MACSYMA.

\section{O.2 SQED FORM FACTORS}

In the SQED case we use the integral functions of Table L.1 but the mas: ratios of eqn. (3.10) become

$$
T_{L, R}=\frac{m_{i_{1}}^{2}}{m_{L}^{2}} \quad R=\frac{t^{2}}{1 m_{1}^{1}} .
$$

We also define

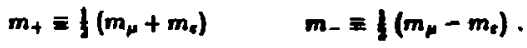

It is then found that

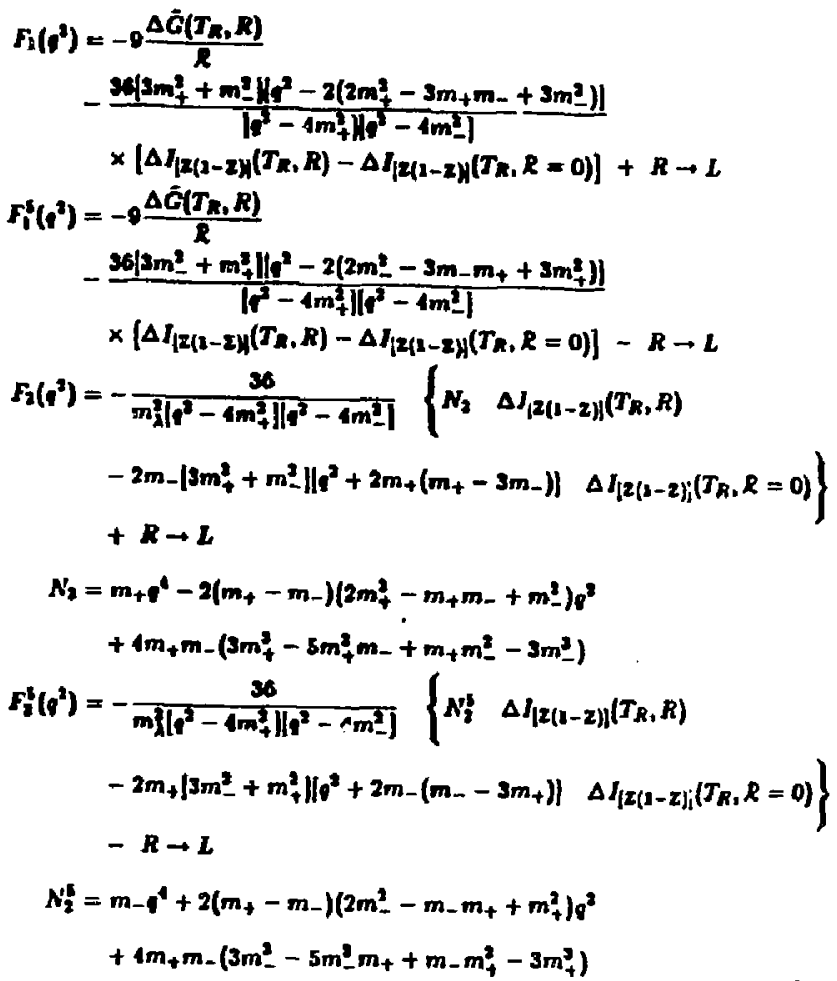


These expressions are valid for an $p^{\prime}$. Note that $F_{i}^{k}$ and $F_{2}^{5}$ are the same $a f_{i}$ and $f_{z}$ sespectively except for $m_{+} \rightarrow m_{-}$. and the relutive sighs of the feft and sight sectors. In deriving these form taclors algebrate simplification was pacilitated by use of the equation

$$
J_{(I)}(T, R)=\frac{1}{\partial R} \bar{C}(T, R)
$$

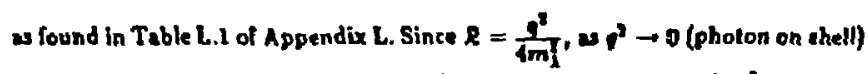
we have $R \rightarrow 0$ and we aee that the $\Delta I_{[z(1-2)]}$ terms in $F_{1}$ and $F_{i}^{\prime}$ explicitly rasish. If is also true that $\vec{C}(T, R)$ varishes at $R \rightarrow 0$ but

$$
\lim _{h \rightarrow 0} \frac{\dot{G}(T, R)}{R} \rightarrow \frac{(1-T)\left(2 T^{2}-7 T+11\right)}{(1-T)^{4}}
$$

This expression is regular of $T=\dot{b}$.

$$
\lim _{T \rightarrow 1} \lim _{R \rightarrow 0} \frac{\dot{G}(T, R)}{R}-4-\frac{3}{2}
$$

Similar considerations enable us to evaluate $(0.5)$ In various regimes of momen. Ium space. Relatively cimple closed-form expressions have been derived for the form factors in the larze and mall If limits. For example

$$
F_{1}\left(y^{2} \rightarrow 0\right) \rightarrow \Delta h_{1}\left(T_{A}\right)-\frac{R}{3} \Delta f_{1}^{0}\left(T_{R}\right)+(R \rightarrow L)
$$

where

$$
\begin{array}{ll}
f_{1}(x)=\frac{(1-x)\left(2 x^{2}-7 x+11\right)+6 i n x}{(1-x)^{2}} & f_{1}(1)=-\frac{3}{2} \\
f^{0}(x)=\frac{(x-1)\left(3 x^{4}-17 x^{2}+13 x^{2}-77 x-12\right)+6 n x \ln x}{(1-x)^{3}} & f^{0}(1)=2
\end{array}
$$

\subsection{EXACT ANALYTIC EXPREssIONS}

The various integral functions mey be expressed in closed form althoush, in practice, little is gained thereby. As an example

$$
\tilde{G}(T, R)=1-\left\{G_{+}\left(U_{+}\right)-G_{+}(L)+G_{-}\left(U_{-}\right)-G_{-}(L)\right\}
$$

with the following tedious delinitions

$$
\begin{aligned}
& G_{*}(x)=\frac{1}{4}\left(\frac{A_{ \pm}}{B_{ \pm}}-C_{ \pm}-D_{\star}\right) \\
& A_{ \pm}=2 \ln 2\left(x^{4} \theta^{2} \pm 4 x^{3} \theta^{3}+x^{2}\left(x^{4}+5 p^{4}\right) \mp 2 x \rho\left(x^{4}+\rho^{6}\right)\right] \\
& +\left|-x^{4} p^{2}+6 x^{2} p^{2}-x^{2} p^{4} \pm 2 z p\left(2 p^{4}-x^{4}\right)+2 p^{2} x^{4}\right| \\
& B_{ \pm}=4 \rho^{2}|z \pm \rho|^{2} \\
& C_{ \pm}=\frac{x^{4}}{2 f^{6}} \ln (\mp x-p) \\
& D_{ \pm}=x^{2}\{2 \ln z \cdot \ln (1 \pm * / \rho)-(i j( \pm z / \rho)\}
\end{aligned}
$$

with $h_{2}$ is the dilogarithmic function defined by

$$
f_{1}(x)=-\int_{0}^{e} \frac{\ln (1-x)}{x} d x
$$

Note that $\operatorname{li}_{3}(x)=-\int \ln (1-x) d(\ln x)$ and thet

$$
\int \frac{\ln x}{x+1} d x=\ln x \ln (1+x)+H_{2}(-x) \text {. }
$$

sat 
Continuine with the definitions:

$$
\begin{aligned}
\rho & =\frac{1-T}{2 R} \\
x & =\sqrt{\rho^{2}-1 / R} \\
L & =\sqrt{\rho^{2}-x^{2}} \\
& =\frac{1}{\sqrt{R}} \\
U_{ \pm} & =\sqrt{(1+\rho)^{2}-x^{2}} \pm 1
\end{aligned}
$$

Similar expressions axist for the other integral function.

\section{REFERENCES}

1. J. Wess and J. Bager, Supersymmetry and Supergyavity, Princeton Univernity Ruas (103).

2. Sakurai, 3.3., Adunced Quanfum Mfechanica, Addison- Wesley, 1980

3. P. Fayet, Nuct. Phyc. Bga (1975) 104.

4. S. Ferrase and E. Remidi, Phys. Lelt. 538 (1974) 347.

b. H. P. Nillet, Phyr. Rep. 110 (1981) 1.

6. R. Barbieri, S. Fersara and C. A. Savoy, Phyre. Lett. U19B (1082) 343.

7. R. Barbieri and L. Maiani, Phys. Lett. U7B (1082) 203.

3. J. Ellis, J. Hagelin and D. V. Nanopoulos, Phys. Lett. 1168 (1982) 283.

D. J. Bailey at Al., Nucl. Phyc. B130 (1070) 1.

10. T. Kinoshite and W. B. Lindquist, Phys. Rev. Lat. 17 (1981) 357. T. Kinohite et al., Phys. Rev. Lett. 52 (1984) 717.

11. M. Levine, StaC-PUb-1170 (1087), submilted to Phys. Rev. D.

12. H. E. Haber and G. L. Kane, Phys. Hep. 117 (1085) 75.

13. J. F. Gunion and H. E. Haber, Nuel. Phys. B272 (1986) 1.

14. R. M. Barnett, Proceedings of the 1985 SLAC Summer Institute (1985) 95.

1S. A. De Rújuls, H. Georti, and 5. L. Glushow, Phyı. Hev. 212 (1975) 147.

16. M. J. Duncan, Phys. Rev. D3i (1085) 1139.

17. V. Ganepathi et al., Phys. Rev. D27 (1953) 579.

18. P. West, Introduction to Supersymmetry and Supergravity, World Scientific (1986).

19. L. Giardello and M. T. Grisaru, Nucl. Phys. Al91 (1982) 65. 
30. Fouth seference of 11 .

21. A. Bartl et d, preprint UWThPh-1986-49.

22. I am indebted to Foward Haber for the ust of a numerical soutine designed to diagonalize the above and calculate the corresponding vertex matrices.

23. T. Appelquist and J. Carazone, Phys. Rev. Du (1075) 2456.

24. J. Ellit and D. V. Nenopoulot, Phyo. Lelt. 10日 (1982) 14.

23. 8. Komemiya, As presented at the Thirteenth Annual SLAC Summer In. stitute on Particle Physics (1095).

26. As reported by William Ford it the Fourteenth Annual SLAC Summer Institute on Pafticle Physics, ..d86.

27. Particle Propertied Dote Booklet, April 1986. From Phys. Lett. 170B (1986).

28. Pat Butchat, A. Weinstein, G. Feldman. Private Comwunications. Proposal for the MARK If at SLAC. CALT.68-1015.

29. S. Weinbere and G. Feinberg, Phys. Rev. Lett. 1 (1959) 111.

30. R. D. Bolton et. Il., Phya. Rer. Lett., 56 (1986) 2421.

31. R. J. Follebeek, Proceedings of I3th SLAC Summer Institule on Particle Physics (1985) 415.

R. J. Hollebeek, SLAC-PUB 3904 (1986).

C. Foarty ol. el., Phyo. Ror. Latt. 5 (1987) 1171.

32. C. Itzykson and J.-B. Zuber, Quentum Field Theory, McGraw-Hill (1980).

33. IV. Paull and F. Villars, Rer. Mod. Phys. 21 (1049) 134.

34. J. D. Bjorten and S. D. Drell, Relativistie Quantum Field, MeGraw-Hill (1065).
35. J. A.Grifols and A. Mendex, Phys. Rev. Das No 7, (1982) 1809

36. P. Ramond, Field Theory A Mfodern Primer, Benjamin/Cummings (1981)

37. W. Gotdon, z. Pbyaik. Sn (1928) 630.

38. P. Fayet, Phys. Lett. 698 (1977) 489.

39. G. Farrar and P. Fayet, Phys. Lett. 76B (1978) 575.

10. S. Dawson. Proceedinge of 13th SLAC Summet Institute on Particle Physics (1985) 157.

11. L. O'Raileartaigh, Nuel. Phys. 896 (1975) 331.

42. D. V. Nanopoulos, Grand Unifieation with and Withoul Supersymmetry and Cosmolopieal Implieations, World Scientific, (1984) 383.

13. P. Fayet, Superaymmetry and Supergravity '84, Proceedings of the Trieste Spring School, World Scientific (1984) 114.

14. An equivalent formulation may be found in the appendix of R. M. Barnett and H. E. Haber, Phys. Rev. Dal (1985) 85.

15. G. Pessarino and M. Veltoran, Nuc. Phya. B160 (1079) 151.

16. Thenks to Pat Butchal for bnally convincing we of this fact.

17. T. D. Lee, Partiele Physies and an Introduction lo Field Theary, Harwood Academic Publisher (1981).

18. J. D. Jeckson, Ctassied Electrodynamies, John Wiley and Sons, second edition (1915).

19. S. J. Brodsky and J. D. Sulliven, Phys. Rev, 156 (1967) 1644. 